\title{
HEMATOPOIETIC CELL
}

TRANSPLANTATION IN

INBORN ERRORS OF

METABOLISM

HURDLES TO ‘REAL’ CURE

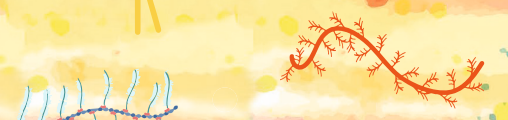





\section{Hematopoietic Cell Transplantation in Inborn Errors of Metabolism \\ Hurdles to 'real' cure}

Brigitte van den Broek 
Hematopoietic Cell Transplantation in Inborn Errors of Metabolism: Hurdles to 'real' cure Thesis with summary in Dutch, Utrecht University

ISBN: 978-94-6375-806-2

Cover Design: Marilou Maes | www.persoonlijkproefschrift.nl

Lay-out: Sanne van Dooremalen en Brigitte van den Broek

Printing: Ridderprint BV | www.ridderprint.nl

The research in this thesis was performed at the University Medical Center Utrecht, Utrecht, The Netherlands. All studies were financially supported by the Sylvia Toth Charity Foundation, Den Haag, The Nerherlands. Additionally, chapter 2 was supported by the National Institute of Health, National Heart, Lung, and Blood Institute (K23HL104575), chapter 5 was supported by a research grant from the Netherlands Organisation for Scientific Research (92003535) and a fellowship grant from the European group for Blood and Marrow Transplantation, and chapter 6 was supported by a grant from Stichting Stofwisselkracht, Haarlem, The Netherlands.

CBrigitte van den Broek, 2020

The copyright of published articles have been transferred to the respective journals. All rights reserved. No part of this thesis may be reproduced, stored in a retrieval system, or transmitted in any other form or by any means, without the permission of the author. 


\title{
Hematopoietic Cell Transplantation in Inborn Errors of Metabolism
}

Hurdles to 'real' cure

\author{
Hematopoietische Stamceltransplantatie \\ in Erfelijke Stofwisselingsziekten \\ Obstakels voor 'echte' genezing \\ (met een samenvatting in het Nederlands)
}

\section{Proefschrift}

ter verkrijging van de graad van doctor aan de

Universiteit Utrecht

op gezag van de

rector magnificus, prof.dr. H.R.B.M. Kummeling, ingevolge het besluit van het college voor promoties in het openbaar te verdedigen op

woensdag 16 december 2020 des middags te 2.30 uur

door

\section{Brigitte Theodora Antonia van den Broek}

geboren op 14 september 1990

te Roosendaal en Nispen 


\section{Promotoren:}

Prof. dr. N.M. Verhoeven-Duif

Prof. dr. J.J. Boelens

\section{Copromotoren:}

Dr. P.M. van Hasselt

Dr. S. Nierkens 
Beoordelingscommissie:

Prof. dr. N.M. Wulffraat

Prof. dr. K.P.J. Braun

Prof. dr. P.M. Hoogerbrugge

Prof. dr. C.D.M. van Karnebeek

Dr. J.J. Jans

\section{Paranimfen:}

Simone Schuilwerve

Annelisa Cornel 



\section{CONTENTS}

Chapter 1 Hematopoietic Cell Transplantation in Inborn Errors of 8 Metabolism: Scope and Intent of the Investigations

\section{Part I In-depth Analyses of Long-term Outcomes after Hematopoietic

Chapter 2 Early and Late Outcomes After Cord Blood Transplantation for

Pediatric Patients with Inherited Leukodystrophies

Chapter 3 Longitudinal Analysis of Ocular Disease in Children with 46 Mucopolysaccharidosis I after Hematopoietic Cell Transplantation

Chapter 4 Hearing Loss in Patients with Mucopolysaccharidoses after 66 Hematopoietic Cell Transplantation; a Longitudinal Analysis

Chapter 5 Quality of Life of Hurler Syndrome Patients After Successful 82 Hematopoietic Cell transplantation

Chapter 6 How to Follow-up Mucopolysaccharidoses Patients After 100 Hematopoietic Cell Transplantation?

Part II

Chapter 7

Pathophysiology of Disease Progression after Hematopoietic

\section{Cell Transplantation}

Salivary $\alpha$-Iduronidase Activity as a Potential New Biomarker for Diagnosing and Monitoring the Effect of Therapy in Mucopolysaccharidosis I

\section{Chapter 8 \\ Chapter 9 \\ Long-Term Effect of Hematopoietic Cell Transplantation on \\ Systemic Inflammation in Mucopolysaccharidoses Patients$$
\text { Hurdles in Treating Hurler's Disease: Potential Routes to Achieve }
$$

a 'Real' Cure

\section{Part III Conclusions and Perspectives}

Chapter 10 Hematopoietic Cell Transplantation in Inborn Errors of 188 Metabolism: Summary, Conlusions, and Perspectives

Chapter $11 \quad$ Nederlandse samenvatting 


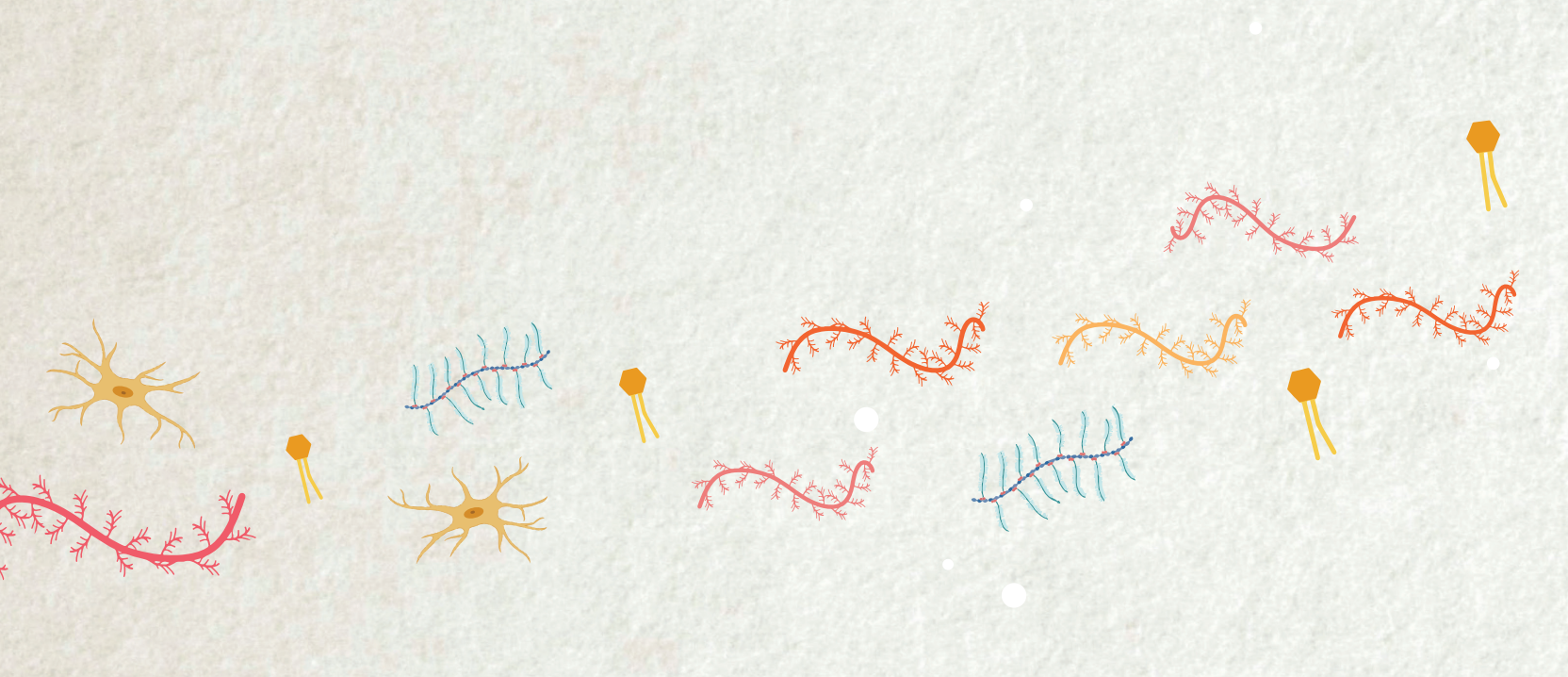



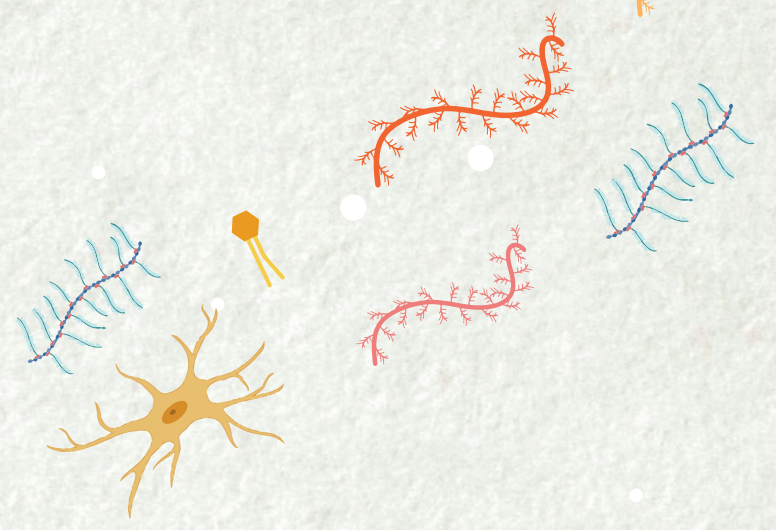

\section{CHAPTER 1}

Hematopoietic Cell Transplantation

in Inborn Errors of Metabolism: Scope and

Intent of the Investigations 


\section{GENERAL INTRODUCTION}

Inborn Errors of Metabolism (IEM) are a heterogeneous group of genetic diseases, including lysosomal and peroxisomal storage diseases caused by defects in genes coding for proteins involved in metabolic pathways. ${ }^{1}$ Most patients with a lysosomal disease appear healthy at birth, however, without treatment all patients will inevitably follow a progressive deterioration and in severe phenotypes ultimately premature death. Allogeneic hematopoietic cell transplantation (HCT) is standard care for a selected group of metabolic diseases and considered optional for others. ${ }^{2}$

\section{The Mucopolysaccharidoses}

The Mucopolysaccharidoses (MPS) are a subfamily of the lysosomal storage disorders (LSD) and comprise seven disorders with a deficiency of one of the 11 lysosomal enzymes needed for the degradation of glycosaminoglycans (GAGs). ${ }^{3}$ GAGs are polysaccharides consisting of repeated disaccharides that vary greatly in length, molecular mass, and sulfation pattern. There are five distinct types of GAGs: heparan sulfate (HS), chondroitin sulfate (CS), dermatan sulfate (DS), keratan sulfates (KS), and hyaluronic acid and all are key elements in connective tissue. Accumulation of GAGs results clinically in a devastating progressive multi-systemic disease with neurocognitive delay, dysostosis multiplex, skeletal malformations, corneal clouding, hearing and vision loss and, if severe, intellectual disability and cardiopulmonary complications which lead to premature death. ${ }^{3}$ MPS- 1 is the IEM for which the largest number of transplantations have been performed and often serves as prototype.

Within the MPS group, transplantation for MPS-1 is the most frequent (about $25 \%$ of all metabolic transplants). The other MPS diseases are less frequently transplanted, both in absolute as in relative terms. Without doubt, HCT improves the overall survival and clinical course of all MPS subtypes, except MPS-3. ${ }^{4}$ Following HCT, rapid reduction of obstructive airway symptoms and organomegaly is seen. Hydrocephalus is generally prevented or stabilized and the incidence of cardiovascular complications and spinal cord compression are significantly reduced. Furthermore, early HCT prevents progressive neurocognitive deterioration. $^{5-14}$ The two main predictors for improved clinical outcomes are timing of transplantation, the earlier the better, and normal enzyme activity levels in leukocytes after HCT. ${ }^{15}$

However, residual disease is more and more recognized and reveals a continuing medical need of the current therapy. For instance, skeletal disease remains a problem in all MPS patients despite treatment with HCT. ${ }^{5}$ Apparently, HCT exerts a variable response within organs. For some, HCT might not deliver sufficient enzyme to completely prevent disease progression. 


\section{Leukodystrophies}

The second largest group of IEM in which HCT is considered a promising therapy, are the Leukodystrophies (LD). ${ }^{16}$ The word leukodystrophy originates from the Greek language and can be literally translated as leuko, white, dys, lack of, troph, growth. Indeed, in LD, the development and maintenance of the myelin sheath of the central nervous system is affected. The myelin sheath is a lipid layer, produced by oligodendrocytes, surrounding the nerve fibers. Its main function is increasing the speed of impulses traveling along the myelinated fiber. Every type of leukodystrophy has a different pathophysiology. However, they all share the mechanism of a gene defect resulting in the disruption of myelin production and maintenance, and, eventually, demyelination. ${ }^{17}$

Metachromatic leukodystrophy (MLD) is caused by a defect in the lysosomal enzyme arylsulfatase A (ARSA), which leads to the accumulation of sulfatides in lysosomes. Sulfatides are the major sphingolipid components of myelin ( $5 \%)$. Therefore, accumulation leads to instability and degradation of myelin and devastating neurological deterioration. ${ }^{18}$ The phenotypic variants are classified by age of onset: late infantile $(<2.5$ years $)$, juvenile (2.5-16 years) or adult ( $>16$ years). The late infantile phenotype is most frequent, with the first presentation before the age of 30 months and rapid deterioration of neurocognitive and motor function followed by death within 2-4 years after diagnosis. ${ }^{19}$ The effect of HCT on clinical outcomes is debatable. If performed early, in pre-symptomatic patients with a juvenile or adult phenotype, onset of symptoms can be delayed. Most patients however, still experience invalidating peripheral neuropathy. ${ }^{20-23}$ The first results of a gene therapy trial, in which late infantile and early juvenile MLD patients were treated with a lentiviral vector encoding ARSA cDNA resulting in supranormal ARSA activity levels in the circulation have been published. ${ }^{24}$ Although promising in the first 7-8 years in the most severe clinical phenotypes, long term follow-up is necessary to evaluate the long term efficacy of gene therapy in MLD patients.

Globoid Cell Leukodystrophy (GLD) or Krabbe disease is caused by a deficiency in the $\beta$-galactosyl-ceramidase gene leading to accumulation of galactosylceramide and progressive central and peripheral demyelination. ${ }^{25}$ Most GLD patients present with irritability, failure to thrive, and progressive loss of motor function in infancy, leading to death within the first 2 years of life. Positive clinical outcomes have been reported for HCT in asymptomatic patients. Sustained engraftment of donor hematopoietic cells led to reduced galactolipid levels, progressive central myelination was facilitated and infants achieved developmental skills and age-appropriate cognitive function. ${ }^{20,26}$ 
Cerebral X-linked adrenoleukodystrophy (X-ALD) is the most frequent LD. It is an X-linked disorder caused by a mutation in the ABCD1 gene. ${ }^{27} \mathrm{X}$-ALD differs from the other LD as it is a disorder of a peroxisomal membrane protein instead of an enzyme. The mutated gene encodes an $\mathrm{ABC}$ transporter which transfers very long chain fatty acids (VLCFA) into the peroxisome for $\beta$-oxidation. The phenotype is heterogeneous including a childhood-onset cerebral form, adult-onset cerebral form, adrenomyeloneuropathy, and adrenal insufficiency without neurological symptoms. Failure of the transfer of VLCFA leads to accumulation in the cytosol of cells in adrenal glands, white matter of the brain, and to excretion in plasma. This accumulation causes inflammatory demyelination of the brain with rapid neurological decline and, when left untreated, mortality. ${ }^{27}$ The exact mechanism, however, is unclear. HCT is indicated in asymptomatic or mildly symptomatic early-onset cerebral X-ALD patients. ${ }^{2,28-30}$ The efficacy is unlikely due to cross-correction. It has been proposed that HCT may arrest the neuroinflammatory demyelinating process by replacing dysfunctional microglia. Encouraging results are also seen in a clinical trial treating patients with gene modified hematopoietic cell therapy, although longer follow-up is needed to fully evaluate the safety and efficacy of this treatment strategy. ${ }^{31}$

\section{Allogeneic Hematopoietic Cell Transplantation}

HCT is based on the principle of cross-correction which was first discovered by Fratantoni et al. ${ }^{32}$ in 1968. A co-culture of skin fibroblasts of Hurler and Hunter patients showed biochemical correction of the deficiencies when the two genotypes were mixed with each other or with healthy cells. Subsequently, in 1981, Hobbs et al. ${ }^{33}$ reported the first hematopoietic cell transplantation in a pediatric patient diagnosed with Hurler syndrome, also known as MPS-1. ${ }^{16}$ Engraftment of donor-derived hematopoietic stem cells in the recipient's bone marrow led to sustained enzyme activity in leukocytes, resolution of specific symptoms (i.e. hepatosplenomegaly), arrest of developmental delay, and significantly prolonged survival with decades. In the years thereafter, more than 500 HCT's worldwide have been performed in Hurler syndrome patients. Besides MPS-1, HCT has been performed in more than 2000 patients affected with other types of MPS, as well as in the LD: MLD, X-ALD, and GLD. ${ }^{16}$ 


\section{SCOPE AND OUTLINE OF THIS THESIS}

Hence, HCT has largely contributed to the improved survival of IEM patients suffering from disorders amenable to HCT with current long-term survival rates of $>90 \%$ upon engraftment. ${ }^{18}$ However, with the increased survival rates, long-term outcomes are evaluated and the areas of residual disease are unfolding. The recognition of residual disease has led to the development of new therapies to improve long-term clinical outcomes. Biomarkers, however, for the evaluation of long-term outcomes are lacking. The objective of this thesis is to provide better insights in the residual disease of transplanted IEM patients and thereby contribute to the improvement of outcomes following HCT. The focus is mainly on Hurler syndrome patients, as this is the metabolic disorder which is transplanted most frequently. To reach this goal, long-term outcomes of HCT in patients with IEM will be analyzed in-depth. Furthermore, hypotheses regarding the pathophysiology of disease progression are explored. Additionally, this thesis may generate insights into new targets for experimental treatments and biomarkers that reflect the residual disease status of patients.

\section{Part 1: In-depth analyses of long-term outcomes after HCT}

The 'Sylvia Toth Center for Multidisciplinary Follow-up after Hematopoietic Cell Transplantation' has $>15$ years of experience with the systematic follow-up of pediatric patients after HCT. Therefore, we are able to describe in-depth analyses of long-term outcomes in these patients. Chapter 2 describes the long-term effects of HCT in leukodystrophies. The majority of IEM patients amenable for HCT, however, are MPS-1 patients. A hallmark feature of MPS-1 is corneal clouding, therefore, chapter 3 comprehends a longitudinal analysis of ocular manifestations in MPS-1 after HCT. Another frequent manifestation of MPS is hearing loss. The effect of HCT on audiological evaluation is described in chapter 4. Besides long-term outcomes, we were interested how MPS affects quality of life in patients who successfully underwent HCT. The results are reported in chapter 5. Finally, for timely recognition of disease progression and in the hope a global standardized followup program will be established so multicenter data of long-term outcomes can be combined and the number of patients in studies can be increased, chapter 6 provides an outline for the systematic evaluation of MPS patients just before HCT and the follow-up thereafter.

\section{Part 2: Pathophysiology of disease progression after HCT}

In part 2 we explore possible explanations for residual disease. In the past years disease progression in IEM after HCT is increasingly acknowledged. Why disease progression only occurs in selective tissues/organs is not entirely clear. One hypothesis, bad penetration in specific tissues, is described in chapter 7 in which enzyme activity in saliva of MPS-1 patients after HCT was studied. Another hypothesis is chronic inflammation, as GAGs have shown to 
directly stimulate the immune system. Therefore, the presence of systemic inflammation and to what extent HCT alters the inflammation status of MPS patients was studied in chapter 8. In chapter 9, the two major hurdles that current treatments are facing are summarized. Moreover, experimental therapies that might be able to overcome these hurdles are reviewed.

\section{Part 3: Conclusions and Perspectives}

Finally, chapter 10 summarizes this thesis and presents the implications of the findings described. Furthermore recommendations for future research are provided to improve clinical outcomes in IEM. 


\section{REFERENCES}

1. El-Hattab AW. Inborn errors of metabolism. Clin Perinatol. 2015; 42(2): 413-439.

2. Tan EY, Boelens JJ, Jones SA, Wynn RF. Haematopoietic Stem Cell Transplantation in Inborn Errors of Metabolism-On Behalf of IEWP-EBMT. Front Pediatr. 2019; 7: 433.

3. Muenzer J. Overview of the mucopolysaccharidoses. Rheumatology. 2011; 50( $\operatorname{suppl} 5)$ : 4-12.

4. Taylor M, Khan S, Stapleton M, et al. Hematopoietic stem cell transplantation for mucopolysaccharidoses; past, present, and future. Biol Blood Marrow Transplant. 2019.

5. Aldenhoven M, Wynn RF, Orchard PJ, et al. Long-term outcome of Hurler syndrome patients after hematopoietic cell transplantation: an international multicenter study. Blood. 2015; 125(13): 2164-2172.

6. Lum SH, Stepien KM, Ghosh A, et al. Long term survival and cardiopulmonary outcome in children with Hurler syndrome after haematopoietic stem cell transplantation. J Inherit Metab Dis. 2017; 40(3): 455-460.

7. Tanaka A, Okuyama T, Suzuki Y, et al. Long-term efficacy of hematopoietic stem cell transplantation on brain involvement in patients with mucopolysaccharidosis type II: a nationwide survey in Japan. Mol Genet Metab. 2012; 107(3): 513-520.

8. Kubaski F, Yabe H, Suzuki Y, et al. Hematopoietic stem cell transplantation for patients with mucopolysaccharidosis II. Biol Blood Marrow Transplant. 2017; 23(10): 1795-1803.

9. Welling L, Marchal JP, van Hasselt P, van der Ploeg AT, Wijburg FA, Boelens JJ. Early Umbilical Cord Blood-Derived Stem Cell Transplantation Does Not Prevent Neurological Deterioration in Mucopolysaccharidosis Type III. 2014: 63-68.

10. Wang J, Luan Z, Jiang H, et al. Allogeneic Hematopoietic Stem Cell Transplantation in Thirty-Four Pediatric Cases of Mucopolysaccharidosis-A Ten-Year Report from the China Children Transplant Group. Biol Blood Marrow Transplant. 2016; 22(11): 2104-2108.

11. Yabe H, Tanaka A, Chinen Y, et al. Hematopoietic stem cell transplantation for Morquio A syndrome. Mol Genet Metab. 2016; 117(2): 84-94.

12. Turbeville S, Nicely H, Rizzo JD, et al. Clinical outcomes following hematopoietic stem cell transplantation for the treatment of mucopolysaccharidosis VI. Mol Genet Metab. 2011; 102(2): 111-115.

13. Behfar M, Dehghani SS, Rostami T, Ghavamzadeh A, Hamidieh AA. Non-sibling hematopoietic stem cell transplantation using myeloablative conditioning regimen 
in children with Maroteaux-Lamy syndrome: A brief report. Pediatr Transplant. 2017; 21(5): 12981.

14. Montaño AM, Lock-Hock N, Steiner RD, et al. Clinical course of sly syndrome (mucopolysaccharidosis type VII). J Med Genet. 2016; 53(6): 403-418.

15. Boelens JJ, Rocha V, Aldenhoven M, et al. Risk factor analysis of outcomes after unrelated cord blood transplantation in patients with hurler syndrome. Biol Blood Marrow Transplant. 2009; 15(5): 618-625.

16. Boelens JJ, Bierings M, Wynn RF. HSCT handbook EBMT/ESH 2012: HSCT for children and adolescents. Paris Eur Sch Haematol. 2012: 1-14.

17. Raymond G V. Leukodystrophy: Basic and Clinical. In: Advances in Neurobiology. Vol 15. 2017: 365-382.

18. Patil SA, Maegawa GHB. Developing therapeutic approaches for metachromatic leukodystrophy. Drug Des Devel Ther. 2013; 7: 729-745.

19. van Rappard DF, Boelens JJ, Wolf NI. Metachromatic leukodystrophy: Disease spectrum and approaches for treatment. Best Pract Res Clin Endocrinol Metab. 2015; 29(2): 261-273.

20. Krivit W, Lockman LA, Watkins PA, Hirsch J, Shapiro EG. The future for treatment by bone marrow transplantation for adrenoleukodystrophy, metachromatic leukodystrophy, globoid cell leukodystrophy and Hurler syndrome. J Inherit Metab Dis. 1995; 18(4): 398-412.

21. Krivit W, Shapiro E, Kennedy W, et al. Treatment of late infantile metachromatic leukodystrophy by bone marrow transplantation. N Engl J Med. 1990; 322(1): 28-32.

22. Krivit W, Peters C, Shapiro EG. Bone marrow transplantation as effective treatment of central nervous system disease in globoid cell leukodystrophy, metachromatic leukodystrophy, adrenoleukodystrophy, mannosidosis, fucosidosis, aspartylglucosaminuria, Hurler, Maroteaux-Lamy, and Sly syn. Curr Opin Neurol. 1999; 12(2): 167-176.

23. Martin HR, Poe MD, Provenzale JM, Kurtzberg J, Mendizabal A, Escolar ML. Neurodevelopmental outcomes of umbilical cord blood transplantation in metachromatic leukodystrophy. Biol Blood Marrow Transplant. 2013; 19(4): 616-624.

24. Sessa M, Lorioli L, Fumagalli F, et al. Lentiviral haemopoietic stem-cell gene therapy in early-onset metachromatic leukodystrophy: an ad-hoc analysis of a nonrandomised, open-label, phase 1/2 trial. Lancet. 2016; 388: 476-487.

25. Pagon RA, Adam MP, Ardinger HH. Krabbe disease. eneReviews 1993. Seattle, WA Univ Washingt.

26. Escolar ML, Poe MD, Provenzale JM, et al. Transplantation of umbilical-cord blood 
in babies with infantile Krabbe's disease. N Engl J Med. 2005; 352(20): 2069-2081.

27. Moser HW, Mahmood A, Raymond G V. X-linked adrenoleukodystrophy. Nat Rev Neurol. 2007; 3(3): 140.

28. Aubourg P, Blanche S, Jambaqué I, et al. Reversal of early neurologic and neuroradiologic manifestations of X-linked adrenoleukodystrophy by bone marrow transplantation. N Engl J Med. 1990; 322(26): 1860-1866.

29. Shapiro E, Krivit W, Lockman L, et al. Long-term effect of bone-marrow transplantation for childhood-onset cerebral X-linked adrenoleukodystrophy. Lancet. 2000; 356: 713-718.

30. Miller WP, Rothman SM, Nascene D, et al. Outcomes after allogeneic hematopoietic cell transplantation for childhood cerebral adrenoleukodystrophy: The largest single-institution cohort report. Blood. 2011; 118(7): 1971-1978.

31. Cartier N, Hacein-Bey-Abina S, Bartholomae CC, et al. Hematopoietic Stem Cell Gene Therapy with a Lentiviral Vector in X-Linked Adrenoleukodystrophy. Science (80- ). 2009; 326: 818-823.

32. Fratantoni JC, Hall CW, Neufeld EF. Hurler and Hunter Syndromes: Mutual Correction of the Defect in Cultured Fibroblasts. Science (80- ). 1968; 162: 570-572.

33. Hobbs JR, Hugh-Jones K, Barrett AJ, et al. Reversal of clinical features of Hurler's disease and biochemical improvement after treatment by bone-marrow transplantation. Lancet (London, England). 1981; 2: 709-712. 


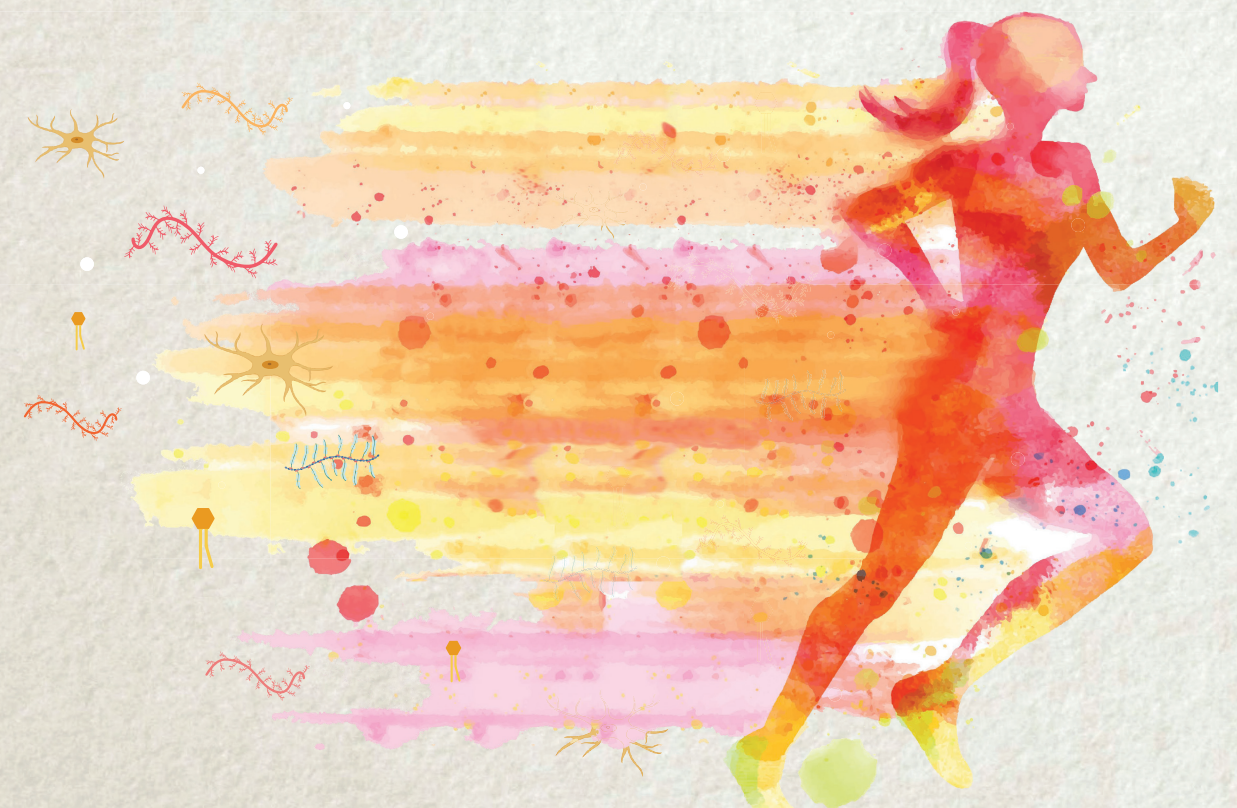




\section{PART I}

In-depth Analyses of Long-term

Outcomes after Hematopoietic

Cell Transplantation 


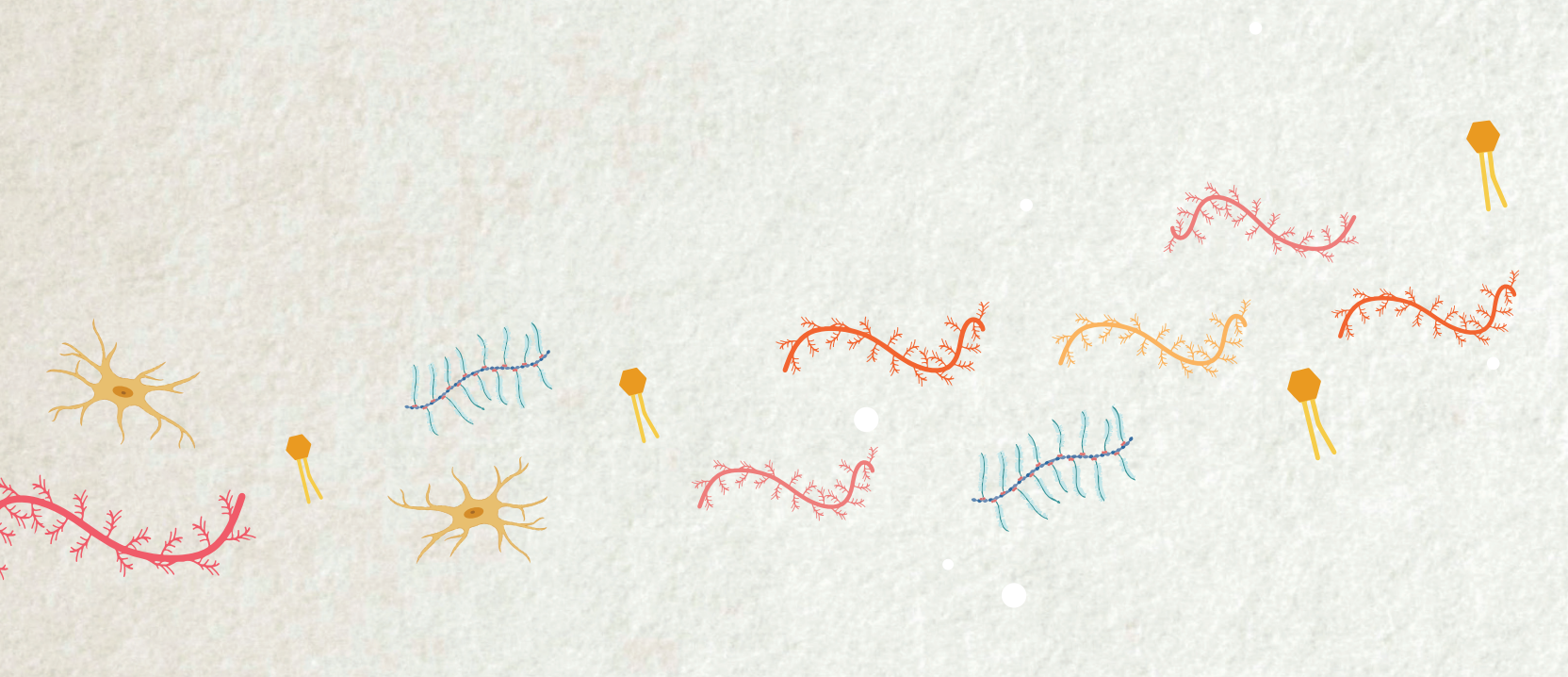



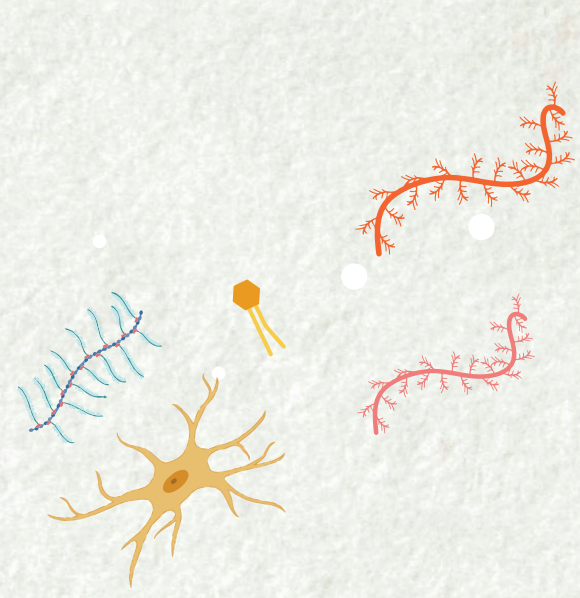

\section{CHAPTER 2}

\section{Early and Late Outcomes After Cord Blood Transplantation for Pediatric Patients with Inherited Leukodystrophies}

Brigitte T.A. van den Broek*

Kristin Page*

Annalisa Paviglianiti*

Janna Hol

Heather Allewelt

Fernanda Volt

Gerard Michel

Miguel Angel Diaz

Victoria Bordon

Tracey O'Brien

*Authors contributed equally
Peter J. Shaw

Chantal Kenzey

Amal Al-Seraihy

Peter M. van Hasselt

Andrew R. Gennery

Eliane Gluckman

Vanderson Rocha

Annalisa Ruggeri

Joanne Kurtzberg*

Jaap Jan Boelens* 


\begin{abstract}
Leukodystrophies (LD) are devastating inherited disorders leading to rapid neurological deterioration and premature death. Hematopoietic stem cell transplantation can halt disease progression for selected LD. Cord blood is a common donor source for transplantation of these patients because it is rapidly available and can be utilized without full HLAmatching. However, precise recommendations allowing care providers to identify patients who benefit from HCT are lacking. In this study, we define risk factors and describe the early and late outcomes of 169 patients with globoid cell leukodystrophy (GLD), X-linked adrenoleukodystrophy (X-ALD) and metachromatic leukodystrophy (MLD) undergoing CBT at an EBMT-center or at Duke University Medical Center from 1996-2013. Factors associated with higher OS included pre-symptomatic status ( $77 \%$ vs. 49\%; $\mathrm{p}=0.006)$, wellmatched ( $\leq 1$ HLA-mismatch) CB units (71\% vs. 54\%; $=0.009)$, and performance status (PS) $>80$ versus $<60$ or $60-80$ ( $69 \%$ versus $32 \%$ and $55 \%$ respectively; $\mathrm{p}=0.003$ ). For patients with a PS $\leq 60(n=20)$ or $60-80(n=24)$ pre-CBT, only $4(9 \%)$ showed improvement. Of the survivors with a PS $>80$ pre-CBT, $50 \%$ remained stable, while $20 \%$ declined to $60-80$ and $30 \%$ to $<60$. Decline was mainly seen during the first year post transplant. Of the pre-symptomatic X-ALD patients, 51\% remained without symptoms at last follow-up. For MLD and GLD combined, this was $18 \%$. Overall, an encouraging OS was found for LD patients after CBT, especially for those who are pre-symptomatic before CBT and received adequately dosed grafts. Early identification and fast referral to a specialized center will hopefully lead to earlier treatment and, subsequently, improved outcomes of these patients.
\end{abstract}




\section{INTRODUCTION}

Leukodystrophies (LD) are a heterozygous group of rare inherited diseases that affect the development and maintenance of brain myelination. Although the age of onset and clinical course varies among this group of diseases, all inherited leukodystrophies are characterized by progressive neurological deterioration and premature death. They often arise from either a lysosomal storage disease (LSD) such as metachromatic leukodystrophy (MLD) and globoid cell leukodystrophy-Krabbe disease (GLD) or a peroxisomal disorder such as X-linked adrenoleukodystrophy (X-ALD). Hematopoietic cell transplantation (HCT) has been shown to arrest or slow disease progression for MLD, GLD and X-ALD, particularly when performed in pre-symptomatic patients or patients with early stage disease. ${ }^{1,2}$ In patients with a LSD, like MLD or GLD, HCT works through engraftment of donor cells that can cross the bloodbrain barrier, providing a source of cellular enzyme replacement through cross-correction of host cells by enzyme-replete donor cells. ${ }^{3}$ Conversely, in X-ALD, a peroxisomal disorder in which the defected protein is not an enzyme but a transporter protein, the exact mechanism of action of HCT is not completely understood.

Umbilical cord blood (CB), related or unrelated, provides an alternative source of hematopoietic stem cells for transplantation. With over two decades of experience, the benefits of $\mathrm{CB}$ have been well described. Particularly relevant to patients with LD, which are rapidly progressive diseases, $\mathrm{CB}$ is readily available allowing for shorter time to transplant. In patients with LD, the early outcomes of umbilical cord blood transplantation (CBT) have been described. ${ }^{4-10}$ These studies suggest that CBT is most beneficial when performed early, preferably before the onset of symptoms. Although it would be ideal to compare the early and late outcomes based on cell source such as performed for Hurler's disease, ${ }^{11}$ this was not possible due to very limited numbers of patients receiving other cell sources leading us to focus on cord blood only. In this report, we describe the results of a collaboration between Eurocord, the European Society for Blood and Marrow Transplantation (EBMT), and Duke University to define risk factors for survival and describe the early and late outcomes in a large cohort of patients with MLD, GLD, and X-ALD treated with CBT.

\section{PATIENTS AND METHODS}

\section{Patients and Data collection}

In this retrospective, multicenter study, patients (children and adults) with leukodystrophies (MLD, GLD or X-ALD) who received related or unrelated donor CBT between September 1996 and August 2013 were included. Data were collected from the Eurocord Registry from 
EBMT centers through standardized questionnaires that included information about the patients, donors, diseases and transplant outcomes. Data on patients from Duke University were collected through similar questionnaires as those used by the Eurocord-EBMT. Missing data were completed by institutional data managers. An additional follow-up questionnaire was developed for long term outcomes and sent out to participating centers. Symptomatic patients were categorized into disease subtype based on age of onset of symptoms, presymptomatic patients were categorized based on age of onset of the index case in the family. MLD patients were classified as late infantile (0-4 years), early juvenile (4-6 years), late juvenile (6-16 years) or adult ( $>16$ years). Patients with GLD were classified as early infantile ( $<6$ months), late infantile (6-11 months), juvenile (1-16 years), or adult ( $>16$ years). Patients with ALD were classified as childhood (0-10 years), adolescent (10-18 years) or adult ( $>18$ years). All patients with ALD showed evident cerebral disease on MRI at time of transplantation. Symptomatic patients were categorized based on age of onset of symptoms, pre-symptomatic patients were categorized based on age of onset of the index case in the family. This study was performed in agreement with the Helsinki Declaration of 1975, revised in 2008. All patients or their legal representatives gave informed consent. Eurocord and the Working Party for Inborn Errors of EBMT approved the European part of this study. In the US, approval was given by the institutional review board of Duke University.

\section{Outcomes of interest}

The outcomes of interest were overall survival (OS) and event-free survival (EFS). OS was defined as the time from transplantation to death. EFS was defined as time from transplantation to last assessment without an event. Events were considered to be autologous reconstitution (documentation of $<5 \%$ donor-derived engraftment), graft failure (lack of neutrophil recovery or transient engraftment of donor cells after transplantation, and/or requirement for a second transplant) or death. All surviving patients were censored at date of last contact.

Other outcomes of interest were neutrophil recovery, defined as the first day of achieving a neutrophil count of $\geq 0.5 \times 10^{9} \mathrm{~L}$ for 3 consecutive days, incidence of acute and chronic graft versus host disease (GvHD), donor chimerism, enzyme level (arylsulfatase A and galactocerebrosidase tested in blood cell lysates for MLD and GLD, respectively), very long chain fatty acid (VLCFA) level tested in blood cell lysates for X-ALD, and long-term outcomes. Acute GvHD grade II-IV at day 100 was diagnosed and graded according to published criteria. ${ }^{12}$ Chronic GvHD at 5 years was graded according to standard criteria ${ }^{13}$ and evaluated in patients who survived at least 100 days with sustained engraftment. Long-term outcomes included performance score (PS), either Lansky or Karnofsky based on patient age, at 12 months and at latest follow-up time point, overall disease status (symptomatic, defined as clinical disease according to the local clinician, or pre-symptomatic, defined as 
no clinical disease according to the local clinician), descriptive neurological status (presence and severity of peripheral neuropathy, presence of seizures, mental development (tested by Bayley Scales of Infant Development, Stanford-Binet Intelligence Scale, WPPSI-Revised, WISC-III), vision (normal, low: $<20 / 60$, blind: $<20 / 400$ ), hearing (normal: $<25 \mathrm{~dB}$ hearing loss, mildly: 26-55 dB hearing loss, severe $>56 \mathrm{~dB}$ hearing loss), and cerebral atrophy observed by magnetic resonance imaging (MRI)), incontinence, independence in daily living, school attendance and school performance. Furthermore, gall bladder disease was registered for patients with $\mathrm{MLD}^{14,15}$ and adrenal insufficiency for patients with X-ALD (as reported by local clinicians).

\section{Statistical analysis}

Probabilities of EFS and OS were calculated using the Kaplan-Meier estimate; the two-sided log-rank test was used for univariate comparisons. Cumulative incidence curves were created for neutrophil recovery, acute and chronic GvHD were analyzed in a competing risk setting. In univariate analyses, variables associated with the recipient (median age at transplant, median weight at time of transplantation, gender, pre-transplant CMV serology status), the disease (type of diagnosis, and median interval time from diagnosis or (in ALD only) first abnormal brain MRI to transplant), the cord blood unit (HLA-disparity, and median collected and infused total nucleated cells and CD34+ cell doses), and the transplant (year of transplant, use of myeloablation, anti-thymocyte globulin (ATG), and the type of GvHD prophylaxis) were considered. Factors associated with a p-value $<0.10$ in univariate analysis and factors considered relevant risk factors were included in multivariate analyses, using Cox proportional hazards for EFS, OS, neutrophil recovery, and GvHD. Subsequently, a stepwise regression analysis was performed using a threshold of 0.05. All statistical analyses were performed using SPSS version 19 (SPSS Inc., Chicago, IL) and R software version 3.32.0 (The R Foundation for Statistical Computing, Vienna, Austria) software packages. Figures were created with GraphPad Prism version 7.02 (GraphPad Software, La Jolla, California, USA).

\section{RESULTS}

\section{Patient, donor, and transplant characteristics}

One hundred and sixty-nine patients were included: 66 MLD, 47 GLD and 56 X-ALD. Overall, the median age at time of CBT was 5.1 years (range, $0.1-43.3$ ); patients with X-ALD were the oldest with a median age of 8.2 years, followed by MLD (4.3 years) and GLD (0.6 years). The majority of the patients received myeloablative conditioning $(97 \%)$ with Busulfan (Bu) / Cyclophosphamide (Cy) as most frequent regimen (83.4\%). Ninety-two 
percent received a single $\mathrm{CB}$ graft, with a median total nucleated cell dose (TNC) of $5.7 \mathrm{x}$ $10^{7}$ cells $/ \mathrm{kg}$ and a median CD34+ dose of $2.1 \times 10^{5} / \mathrm{kg}$. A cyclosporine-based regimen was used as GvHD prophylaxis in $97 \%$ of the patients and 96\% received ATG as serotherapy. Median follow-up for survivors was 76 months (range 3-211). Patient, donor, and transplant characteristics are shown in Table 1.

\section{Neutrophil and platelets recovery and chimerism}

The cumulative incidence of neutrophil engraftment at day 60 was $86.3 \%$ (range $81.2 \%$ 91.7\%; Table 2) with a median time to engraftment of 21 days (range, 11-83). In univariate analysis, factors associated with higher probability of neutrophil recovery included: shorter interval between diagnosis and CBT ( $\leq 2.84$ months: $91.5 \%$ vs. $>2.84$ months: $80.4 \%, p=0.01)$, higher infused CD34+ cell dose ( $\leq 2.05 \times 10^{5} / \mathrm{kg}: 82.7 \%$ vs. $\left.>2.05 \times 10^{5} / \mathrm{kg}: 88.6 \%, \mathrm{p}=0.05\right)$ and higher infused TNC ( $\leq 5.73 \times 10^{7} / \mathrm{kg}: 82.7 \%$ vs. $\left.>5.73 \times 10^{7} / \mathrm{kg}: 88.9 \%, \mathrm{p}=0.008\right)$. The cumulative incidence of platelet recovery was $68 \pm 6 \%$ at day 180 and the median time to platelet recovery $(>20,000 / \mu \mathrm{l})$ was 52 days (range, $13-200$ days). Twenty patients $(12 \%)$ experienced either autologous reconstitution $(n=6 ; 3.5 \%)$ or secondary graft failure $(n=14$; $8 \%$ ) during the first three months after CBT. Thirteen of these patients subsequently died due to disease progression $(n=3)$ or graft failure $(n=10)$. Eight patients received a second transplant using CB $(n=5)$, peripheral blood stem cells $(n=1)$ or not specified $(n=2)$ as the donor source. Of these, six patients survived long-term and two died (1 due to disease progression, and 1 transplant-related mortality). One patient with autologous recovery survived long-term without a second transplant. Chimerism at day 100 was available for 125 out of 148 engrafted patients. Full chimerism was achieved in 104 (83.2\%) of the patients, and mixed chimerism in $21(16.8 \%)$. Chimerism at last assessment was available for all 100 survivors (full chimerism $\mathrm{n}=87$; mixed chimerism $\mathrm{n}=13$ ). Of patients who were "alive and engrafted", normal enzyme levels were found in $94.8 \%$ and $96.6 \%$, for MLD and GLD, respectively.

\section{Acute and chronic GvHD}

The cumulative incidence of acute GvHD grades II-IV and III-IV at day 100 was $35.3 \% \pm 7 \%$, and $20.2 \% \pm 8 \%$ (table 2), respectively. Univariate analysis showed that a longer interval between diagnosis and CBT was associated with a higher probability of acute GvHD grade II-IV (47.6\% for $\leq 2.84$ and $25 \%$ for $>2.84 ; \mathrm{P}=0.002)$. The cumulative incidence of chronic GvHD at 5 years was $30.2 \% \pm 5 \%$ (15 extensive and 28 limited). In univariate analysis, higher PS at the time of CBT was associated with a higher incidence of chronic GvHD (12.1\% for $\mathrm{PS} \leq 60,20.8 \%$ for PS $60-80$ and $41.6 \%$ for $\mathrm{PS}>80 ; \mathrm{p}=0.009 ; 65 \%$ was limited chronic GvHD). Only six of the patients with chronic GvHD and a lower PS before CBT ( $n=2$ for $<60$ and $n=4$ for $60-80$ ) are still alive at latest follow-up. 


\section{OS, EFS, and causes of death}

The 1-year and 6-year OS among all patients was $73 \pm 3 \%$ and $61 \pm 4 \%$ (Figure 1a), respectively, with a median follow-up of 76 months (range, 12-211). Similar OS was observed for the three LD types. Improved OS was observed in patients with late-onset disease in MLD and early-onset disease in patients with GLD or X-ALD (Supplemental Figure 1), although this should be interpret with caution since classification of patients might have been based on

Table 1. Baseline patient, donor, and transplantation characteristics.

\begin{tabular}{|c|c|c|}
\hline \multicolumn{3}{|l|}{ Baseline characteristics* } \\
\hline Patient characteristics & $\mathbf{n}$ & $\%$ \\
\hline Overall & 169 & \\
\hline MLD & 66 & 39.1 \\
\hline GLD & 47 & 27.8 \\
\hline X-ALD & 56 & 33.1 \\
\hline Child $(<18$ yrs)/adult & $161 / 8$ & $95.3 / 4.7$ \\
\hline Gender (male/female) & $118 / 51$ & $69.8 / 30.2$ \\
\hline \multirow[t]{2}{*}{ CMV serology (negative) } & 127 & 77.9 \\
\hline & Median & Range \\
\hline Weight (kg) & 19.5 & $2.74-75.0$ \\
\hline \multicolumn{3}{|l|}{ Age at SCT (yrs) } \\
\hline Overall & 5.1 & $.1-43.3$ \\
\hline MLD & 4.3 & $.1-22.7$ \\
\hline GLD & .6 & $.1-16.7$ \\
\hline X-ALD & 8.2 & $2.4-43.3$ \\
\hline \multicolumn{3}{|l|}{ Interval diagnosis-transplant (mo) } \\
\hline Overall & 2.8 & $.6-147.9$ \\
\hline MLD & 3.0 & $.6-70.3$ \\
\hline GLD & 1.6 & $.6-60.0$ \\
\hline X-ALD & 5.4 & $.6-147.9$ \\
\hline \multicolumn{3}{|l|}{ Performance score pre SCT } \\
\hline$<60$ & 29 & 19.6 \\
\hline $60-80$ & 29 & 19.6 \\
\hline$>80$ & 90 & 60.8 \\
\hline Donor characteristics & $\mathbf{n}$ & $\%$ \\
\hline $\mathrm{sCB}$ & 156 & 92.3 \\
\hline $\mathrm{dCB}$ & 13 & 7.7 \\
\hline Unrelated/related & $168 / 1$ & $99.4 / .6$ \\
\hline \multicolumn{3}{|l|}{ HLA-matching } \\
\hline $6 / 6$ & 27 & 18.1 \\
\hline $5 / 6$ & 56 & 37.6 \\
\hline $4 / 6$ & 65 & 43.6 \\
\hline $3 / 6$ & 1 & .7 \\
\hline \multicolumn{3}{|l|}{$\mathrm{CB}$ cell dose } \\
\hline Collected NC (.107/kg) & 8.0 & 2.1-67.2 \\
\hline Collected CD34+ $(.105 / \mathrm{kg})$ & 2.7 & $.01-28.5$ \\
\hline Infused NC $(.107 / \mathrm{kg})$ & 5.7 & $1.2-50.3$ \\
\hline Infused CD34+ $(.105 / \mathrm{kg})$ & 2.1 & $.01-32.4$ \\
\hline
\end{tabular}


Table 1. Baseline patient, donor, and transplantation characteristics (continued).

\begin{tabular}{|c|c|c|}
\hline Transplantation characteristics & n & $\%$ \\
\hline \multicolumn{3}{|l|}{ Conditioning regimen } \\
\hline MAC & 163 & 97 \\
\hline $\mathrm{Bu} / \mathrm{Cy} /(\mathrm{TT})$ & $135(1)$ & \\
\hline Bu/Fluda/(Cy)(Mel)(TT) & $9(4)(3)(3)$ & \\
\hline Fluda/TT/Mel & 3 & \\
\hline $\mathrm{Bu} / \mathrm{Mel} /$ Other & 1 & \\
\hline $\mathrm{TBI} / \mathrm{Cy}$ & 4 & \\
\hline RIC & 5 & 3 \\
\hline Fluda/Mel & 1 & \\
\hline Mel/Other & 1 & \\
\hline Fluda/Other & 2 & \\
\hline Cy/Fluda/TBI & 1 & \\
\hline \multicolumn{3}{|l|}{ GvHD prophylaxes } \\
\hline CsA-based & 156 & 96.9 \\
\hline+ Steroids & 34 & \\
\hline$+\mathrm{MMF}$ & 29 & \\
\hline+ Mtx & 4 & \\
\hline Tacrolimus-based & 5 & 3.1 \\
\hline$+\mathrm{MMF}$ & 4 & \\
\hline+ Mtx & 1 & \\
\hline \multirow[t]{2}{*}{ Serotherapy (ATG before day 0 ) } & 162 & 96.4 \\
\hline & Median & Range \\
\hline Year of HCT & 2006 & $1996-2013$ \\
\hline Follow-up post $\mathrm{SCT}^{1}(\mathrm{mo})$ & 76.3 & $3.3-211$ \\
\hline \multicolumn{3}{|c|}{$\begin{array}{l}\mathrm{sCD} \text {, single cord blood; dCB, double cord blood; ATG, anti-thymocyte globulin; Bu, busalfan; Csa, } \\
\text { cyclosporine; } \mathrm{Cy} \text {, cyclophosphamide; Fluda, fludarabine; MAC, myoablative conditioning; Mel, } \\
\text { melphalan; MMF, mofetil; NC, neutrophil count; RIC, reduced intensity conditioning; SCT, stem cell } \\
\text { transplantation; TT, thiotepa }\end{array}$} \\
\hline \multicolumn{3}{|c|}{${ }^{1}$ Median follow-up of survivors } \\
\hline \multicolumn{3}{|c|}{${ }^{*} \mathrm{CB}$ transplantation was performed in the following centres: Duke (102), Utrecht (11), Marseille (6), } \\
\hline \multicolumn{3}{|c|}{ Australia (6), Saudi Arabia (5), Madrid (4), Gent (4), Brussels (4), Paris (4), Milano (2), Murcia (2), } \\
\hline \multicolumn{3}{|c|}{ Barcelona (2), Montréal (2), Budapest (2), Leiden (1), Leuven (1), Jerusalem (1), Porto (1), Hannover (1), } \\
\hline Lisboa (1), Berlin (1), Manchester (1), & ncy (1), Israel ( & Bratislava (1) \\
\hline
\end{tabular}

an index case. In univariate analysis, several clinical factors were associated with improved OS including: recipients of $5 / 6$ or $6 / 6$ HLA matched grafts ( $71 \%$ vs. $54 \%$ for $3-4 / 6$ HLA matched grafts, $\mathrm{p}=0.009)$, absence of cerebral atrophy on MRI prior to CBT ( $68 \%$ vs. $35 \%$ for patients with cerebral atrophy; $\mathrm{p}<0.001$ ), PS before CBT $>80$ (69\% compared to $55 \%$ and $32 \%$ for patients with PS $60-80$ or $<60$, respectively, $\mathrm{p}=0.003$ ). Pre-symptomatic or mildly affected patients also experienced higher OS as compared to symptomatic patients ( $77 \%$ vs. $49 \%, p=0.006$; Figure $2 \mathrm{a}$ and $2 \mathrm{~b}$ ). OS was not influenced by year of transplantation (median $2006 ; 59 \pm 5 \%$ vs. $63 \pm 7 \%$, p $=0.44$ ). Further analysis revealed that differences in OS between pre-symptomatic and symptomatic patients were most notable in GLD and X-ALD patients (GLD: $78 \%$ vs. $36 \%, \mathrm{p}=0.02$; X-ALD: $88 \%$ vs. $47 \%$, $\mathrm{p}=0.02$; and MLD $70 \%$ vs. $62 \%$, 

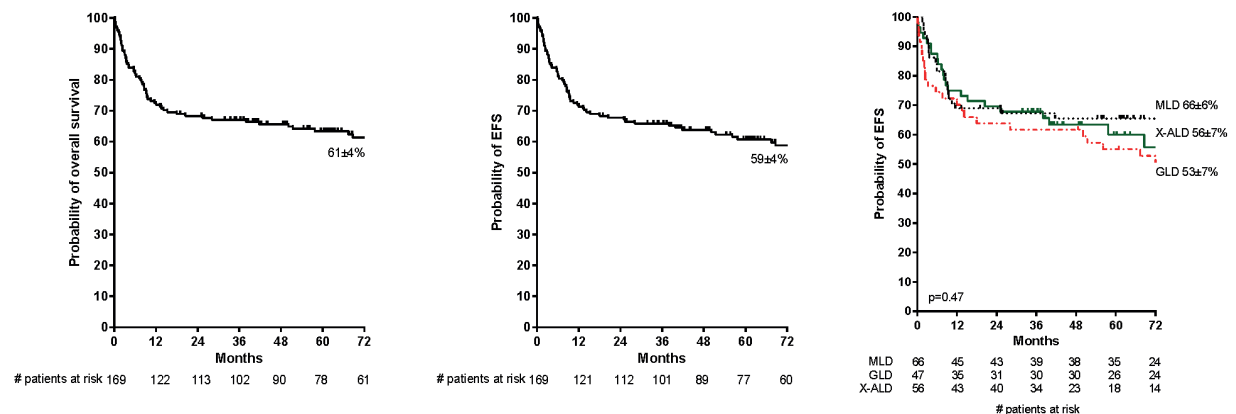

Figure 1. 6-Year overall and event-free survival. (a) OS; (b) EFS; (c) EFS per LD type. Events were considered to be autologous reconstitution, graft failure or death.

$\mathrm{p}=0.64)$. In multivariate analysis, recipients of $5 / 6$ or $6 / 6$ HLA-matched grafts $(\mathrm{HR}=1.8, \mathrm{CI}$ $95 \% 1.0-3.3, \mathrm{p}=0.04)$ as well as pre-symptomatic disease status ( $\mathrm{HR}=2.0, \mathrm{CI} 95 \%$ 1.0-3.7, $\mathrm{p}=0.04$ ) were significantly associated with higher OS at 6-years.

In the overall cohort, the 1 -year EFS was $72 \pm 4 \%$ and the 6 -year EFS $59 \pm 4 \%$ (Figure $1 \mathrm{~b}$ ). Although there was a trend towards improved EFS for patients with pre-symptomatic disease and those who received 5 or 6/6 HLA-matched grafts, no clinical factors were significantly associated with EFS in multivariate analysis (Table 3). Notably, pre-symptomatic GLD patients had significantly increased EFS as compared to symptomatic GLD patients $(78 \%$ vs. $32 \%, \mathrm{p}=0.009$; Figure 2).
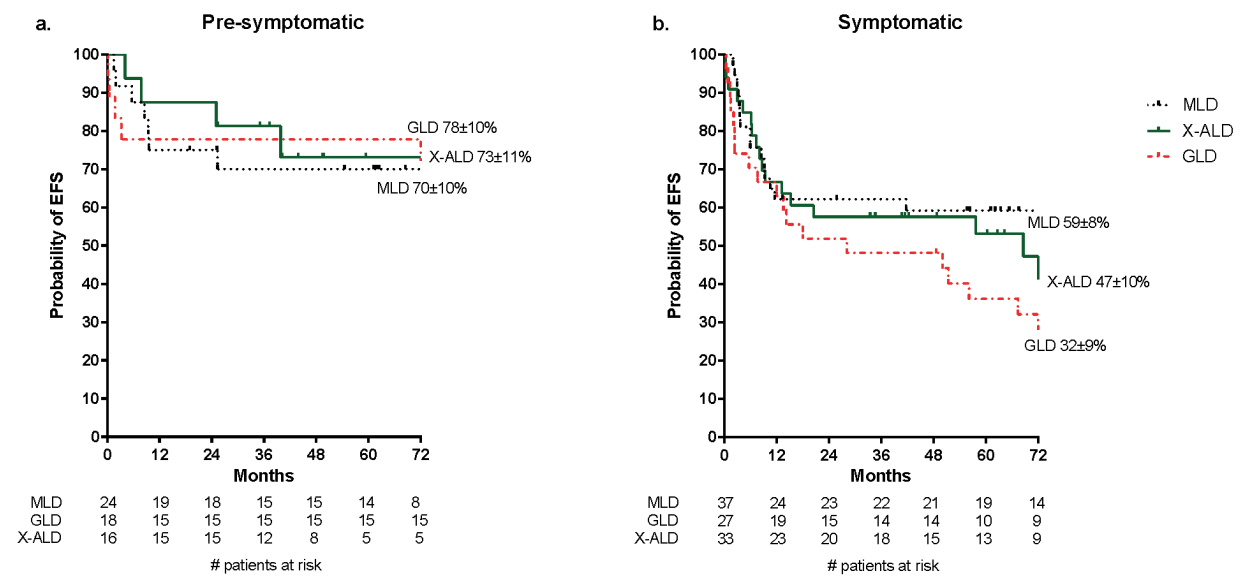

Figure 2. EFS for pre-symptomatic (a) and symptomatic (b) patients at time of CBT. Events were considered to be autologous reconstitution, graft failure or death. 
Table 2. Primary and secondary endpoints.

\begin{tabular}{|c|c|c|}
\hline Primary endpoints & $\%$ & Events (n) \\
\hline 6-y OS Overall & 61 & \\
\hline MLD & 67 & \\
\hline $\mathrm{X}-\mathrm{ALD}$ & 59 & \\
\hline GLD & 55 & \\
\hline 6-y EFS Overall & 59 & \\
\hline MLD & 66 & \\
\hline $\mathrm{X}-\mathrm{ALD}$ & 56 & \\
\hline GLD & 53 & \\
\hline \multicolumn{3}{|l|}{ Secondary endpoints } \\
\hline Neutrophil engraftment (day 60$)^{1}$ & 86.3 & \\
\hline Platelet engraftment (day 180$)^{2}$ & 68 & \\
\hline Graft failure (primary/secondary) & $3.6 / 8.3$ & $6 / 14$ \\
\hline \multicolumn{3}{|l|}{ Chimerism (within 100 days) } \\
\hline Full donor & 83.2 & 104 \\
\hline Mixed & 16.8 & 21 \\
\hline CIF of acute GvHD (stage II-IV) & 35.3 & 59 \\
\hline Stage III-IV & 20.2 & 21 \\
\hline CIF of chronic GvHD at 5 yrs & 30.2 & 44 \\
\hline Limited/extended & & $28 / 15$ \\
\hline \multicolumn{3}{|l|}{ CIF, Cumulative incidence function } \\
\hline \multicolumn{3}{|l|}{${ }^{1}$ Median 21, range $11-83$} \\
\hline${ }^{2}$ Median 52, range $13-200$ & & \\
\hline
\end{tabular}

Sixty-nine patients died within 6 years after CBT (MLD n=23; GLD n=25; X-ALD n=21). Forty-three patients died from transplant-related causes (infection: $\mathrm{n}=18$, GvHD: $\mathrm{n}=9$, pulmonary disease: $n=7$, multisystem organ failure: $n=4$, or other: $n=5$ ), and 21 from disease progression. Additional causes of death are provided in supplemental table 1.

\section{Functional and disease related outcomes}

To compare functional outcomes after CBT, we utilized the pre- and post-transplant PS and retrospectively assigned disease status based on symptoms present at the time point of interest. Pre- and post-transplant PS were available for 119 patients. Amongst survivors with normal pre-transplant functional status (PS $>80$ ), $50 \%$ had a PS $>80$ at last follow-up (median 69 months, range 12-211; Figure 3), while the remaining showed a decline in PS to 60-80 $(20 \%)$ or $<60(30 \%)$. For patients with lower pre-transplant PS ( $\leq 60 n=20 ; 60-80 n=24)$, only 4 patients demonstrated improved PS after CBT and at last follow-up. When declines in PS occurred after CBT, it most commonly occurred during the first year and was stable afterwards (Figure 3 and 4). Changes in performance scores according to LD type are shown in Figure 4. In addition, changes in performance score according to the different clinical phenotypes per LD can be found in Supplemental Figure 2. 
Table 3. Multivariate predictors of 6-year OS and EFS.

\begin{tabular}{|c|c|c|c|}
\hline & \multicolumn{3}{|c|}{ 6-Year OS } \\
\hline & HR & $95 \% \mathrm{CI}$ & P-value \\
\hline HLA 0 - 1 versus $\geq 2$ HLA mismatches & 1.8 & $1.0-3.3$ & 0.04 \\
\hline $\begin{array}{l}\text { Pre-symptomatic versus } \\
\text { symptomatic }\end{array}$ & 2.0 & $1.0-3.7$ & 0.04 \\
\hline \multirow[t]{3}{*}{$\begin{array}{l}\text { Collected } \mathrm{TNC} \geq 5.0 .105 \text { versus } \\
\text { collected } \mathrm{TNC}<5.0 .105\end{array}$} & 1.5 & $0.7-3.2$ & 0.34 \\
\hline & \multicolumn{3}{|c|}{ 6-Year EFS } \\
\hline & HR & $95 \% \mathrm{CI}$ & P-value \\
\hline HLA $0-1$ versus $\geq 2$ HLA mismatches & 1.7 & $1.0-2.9$ & 0.059 \\
\hline $\begin{array}{l}\text { Pre-symptomatic versus } \\
\text { symptomatic }\end{array}$ & 1.8 & $1.0-3.2$ & 0.057 \\
\hline $\begin{array}{l}\text { Collected } \mathrm{TNC} \geq 5.0 .105 \text { versus } \\
\text { collected } \mathrm{TNC}<5.0 .105\end{array}$ & 1.6 & $0.7-3.4$ & 0.227 \\
\hline
\end{tabular}
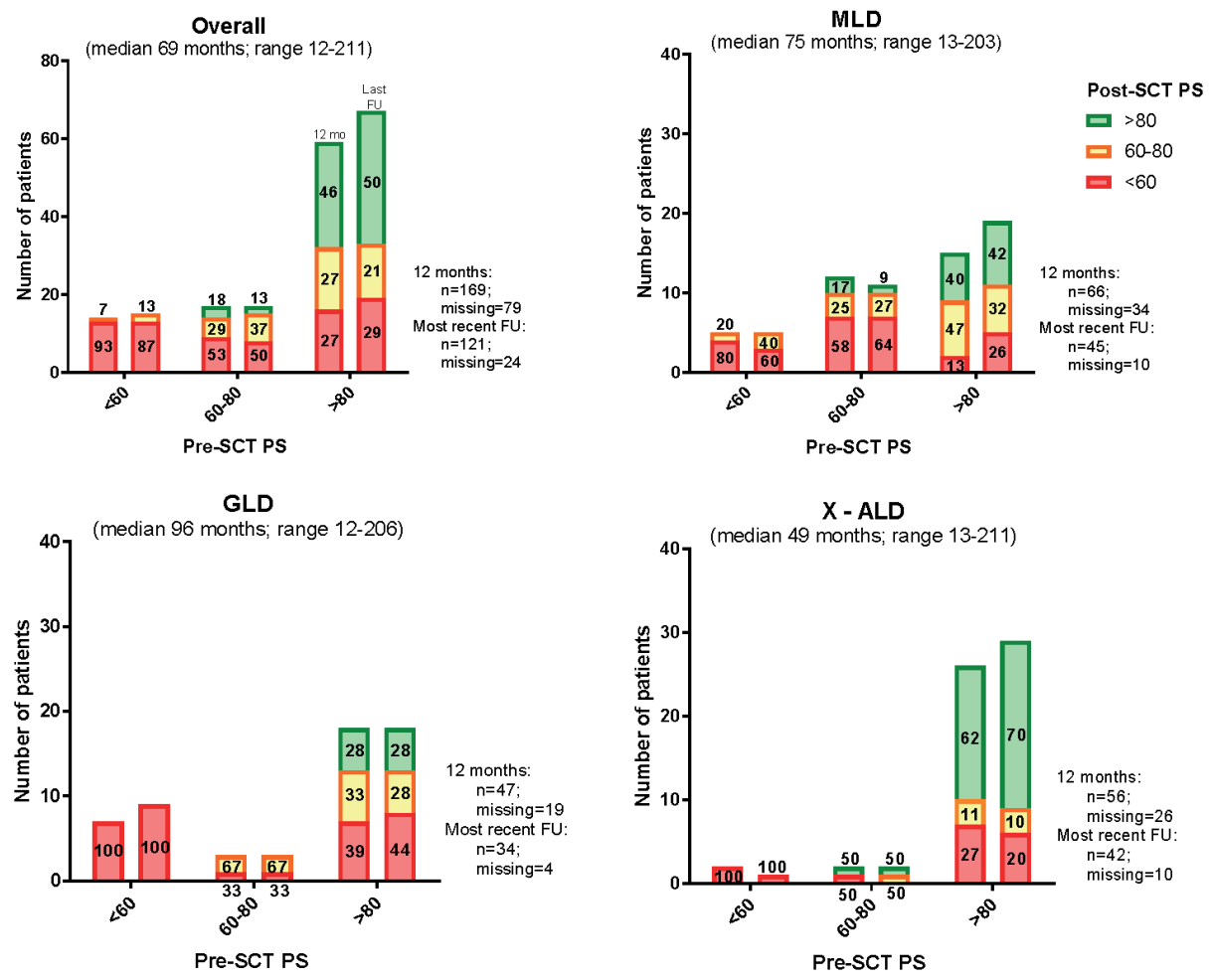

Figure 3. Performance score before and after CBT at $\mathbf{1 2}$ months and at most recent follow-up. Most recent follow-up only consists of patients who survived $\geq 12$ months. Percentages show proportions of number of patients of corresponding pre-HCT PS. All paired bars follow the sequence 1 year and most recent follow-up. 
Data on disease related outcomes was available for all patients pre-CBT $(n=169)$ and for 98 patients who survived more than 1-year post-CBT (Table 4). In patients with enzyme testing results post-CBT, 93\% and 95\% of patients for MLD and GLD, respectively, achieved normal enzyme levels (arylsulfastase A or galactocerebrosidase) according to local references. Considering patients with LSDs (i.e. MLD and GLD), $40 \%$ and $18 \%$ of patients were pre-symptomatic pre- and post-CBT, respectively (Table $4 \mathrm{a}$ ). Of the pre-symptomatic patients, $62 \%$ remained without symptoms at last follow-up. Vision and hearing stabilized or improved in the majority of patients after CBT (94\% and 96\%, respectively). Seizures were reported in $24 \%$ of patients post-CBT. Neuropathy, as detected by clinical exam or symptoms, was absent or mild in $61.5 \%$ of patients pre-CBT, and remained stable or improved in $72 \%$ post-CBT. Data on school performance was available for most patients alive at latest follow-up post-CBT (81\%). In this primarily pediatric cohort, $28 \%$ were identified as having age appropriate school performance. Additional educational support was provided to $71 \%$ of patients with available data. Gallbladder disease was observed in $12 \%(n=3)$ of patients with MLD after CBT. All three patients required cholecystectomy. Comparatively, patients with X-ALD appear to have better late outcomes (Table 4b). Of pre-symptomatic patients $100 \%$ remained without symptoms at last follow-up. Neuropathy was uncommon (absent

a
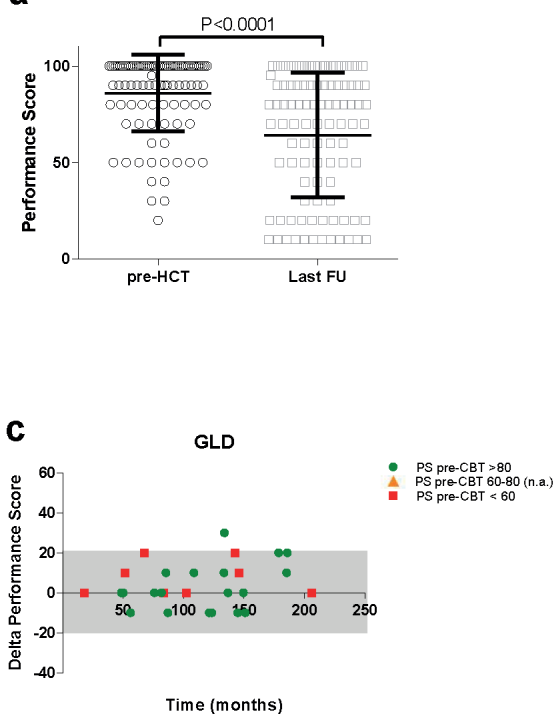

b

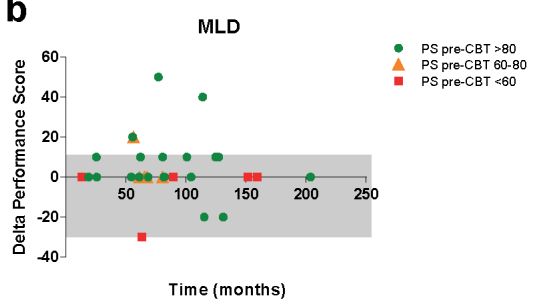

d

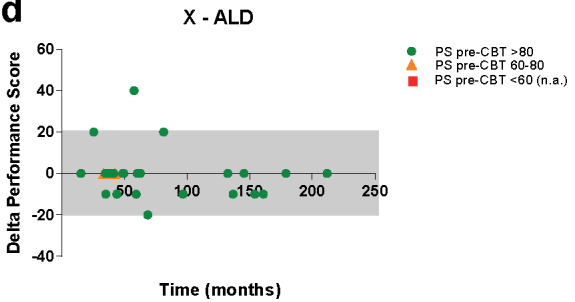

Figure 4. Evolution of performance score before and after CBT. (a) Individual PS of patients with $\geq 12$ months survival before and at last follow-up after CBT; (b)(c)(d) Delta of PS at 12 months and at last follow-up per disease; a positive delta is associated with decline in PS. (b: MLD; c: GLD; $d$ : X-ALD). Legends provide color coding for pre-CBT performance score; n.a., not applicable. 
Table 4a. Functional outcome descriptives pre-HCT and at most recent follow-up in MLD and GLD

\begin{tabular}{|c|c|c|c|c|c|}
\hline \multicolumn{3}{|c|}{ Pre-HCT $(n=113)$} & \multicolumn{3}{|c|}{$\begin{array}{c}\text { Most recent follow-up } \\
(\mathrm{md}=\mathbf{8 7} \text { months, range } 13-206 ; n=63)\end{array}$} \\
\hline Patient characteristics & n (\%) & Missing & Patient characteristics & n (\%) & Missing \\
\hline Enzyme levels & & & Enzyme levels & & \\
\hline MLD (arylsulfatase A) & & 12 & MLD (arylsulfatase A) & & 11 \\
\hline Low & $54(100)$ & & Normal & $28(93.3)$ & \\
\hline Normal & $0(0)$ & & & & \\
\hline GLD (galactocerebrosidase) & & 4 & GLD (galactocerebrosidase) & & 3 \\
\hline Low & $43(100)$ & & Normal & $18(94.7)$ & \\
\hline Normal & $0(0)$ & & & & \\
\hline Presymptomatic & $42(39.6)$ & 7 & Presymptomatic & $10(18.2)$ & 8 \\
\hline Mental development & & & Mental development & & \\
\hline Cognitive & & 40 & Cognitive & & 25 \\
\hline Very low & $16(21.9)$ & & Very low & $15(39.5)$ & \\
\hline Low & $18(24.7)$ & & Low & $8(21.0)$ & \\
\hline Average & $34(46.6)$ & & Average & $15(39.5)$ & \\
\hline Above average & $5(6.8)$ & & & & \\
\hline Motor & & 39 & Motor & & 52 \\
\hline Very low & $16(21.6)$ & & Very low & $10(90.9)$ & \\
\hline Low & $19(25.7)$ & & Low & $0(0)$ & \\
\hline Average & $34(45.9)$ & & Average & $1(9.1)$ & \\
\hline Above average & $5(6.8)$ & & Above average & $0(0)$ & \\
\hline Seizures (present) & $8(7.1)$ & 7 & Seizures (present) & $13(23.6)$ & 8 \\
\hline Neuropathy & & 9 & Neuropathy & & 9 \\
\hline Absent & $36(34.6)$ & & Stable & $37(68.5)$ & \\
\hline Mild & $28(26.9)$ & & Improved & $2(3.7)$ & \\
\hline Severe & $40(38.5)$ & & Worsened & $15(27.8)$ & \\
\hline Vision & & 15 & Vision & & 11 \\
\hline Low & $10(10.2)$ & & Stable & $47(90.4)$ & \\
\hline Blind & $2(2.0)$ & & Improved & $2(3.8)$ & \\
\hline Normal & $86(87.8)$ & & Worsened & $3(5.8)$ & \\
\hline Hearing & & 14 & Hearing & & 11 \\
\hline Mildly affected & $6(6.1)$ & & Stable & $48(92.3)$ & \\
\hline Severely affected & $2(2.0)$ & & Improved & $1(1.9)$ & \\
\hline Normal & $91(91.9)$ & & Worsened & $3(5.9)$ & \\
\hline School attendance* & & 9 & School attendance* & & 6 \\
\hline Regular & $18(47.4)$ & & Regular & $11(21.6)$ & \\
\hline Additional support & $14(36.8)$ & & Additional support & $36(70.6)$ & \\
\hline No attendance & $6(15.8)$ & & No attendance & $4(7.8)$ & \\
\hline School performance* & & 15 & School performance* & & 10 \\
\hline Appropriate for age & $25(75.8)$ & & Appropriate for age & $15(35.8)$ & \\
\hline Lower & $8(24.2)$ & & Lower & $27(64.2)$ & \\
\hline Independence & & 16 & Independence & & 9 \\
\hline Appropriate for age & $52(53.6)$ & & Appropriate for age & $15(27.8)$ & \\
\hline Needs assistance & $45(46.4)$ & & Needs assistance & $39(72.2)$ & \\
\hline Continence (incontinent) & $59(65.6)$ & 23 & Continence (incontinent). & $21(38.9)$ & 9 \\
\hline Gallbladder disease ${ }^{* *}$ (present) & $9(27.3)$ & 33 & Gallbladder disease** (present) & $3(11.5)$ & 17 \\
\hline
\end{tabular}

*only for pediatric patients; ${ }^{* *}$ only for MLD 
Table 4b. Functional outcome descriptives pre-HCT and at most recent follow-up in X-ALD patients outcome descriptives pre-HCT and at most recent follow-up

\begin{tabular}{|c|c|c|c|c|c|}
\hline \multicolumn{3}{|c|}{ Pre-HCT $(n=56)$} & \multicolumn{3}{|c|}{$\begin{array}{c}\text { Most recent follow-up } \\
(\mathrm{md}=49 \text { months, range } 25-211 ; \mathrm{n}=35)\end{array}$} \\
\hline Patient characteristics & n (\%) & Missing & Patient characteristics & n (\%) & Missing \\
\hline Enzyme levels & & & Enzyme levels & & \\
\hline X-ALD (free fatty) & & 17 & X-ALD (free fatty) & & 18 \\
\hline Normal & $0(0)$ & & Normal & $1(5.9)$ & \\
\hline High/elevated & $39(100)$ & & High/elevated & $16(94.1)$ & \\
\hline Presymptomatic & $16(32.7)$ & 7 & Presymptomatic & $15(51.7)$ & 6 \\
\hline Mental development & & & Mental development & & \\
\hline Cognitive & & 18 & Cognitive & & 22 \\
\hline Very low & $0(0)$ & & Very low & $1(7.7)$ & \\
\hline Low & $10(26.3)$ & & Low & $2(15.4)$ & \\
\hline Average & $22(57.9)$ & & Average & $7(53.8)$ & \\
\hline Above average & $6(15.8)$ & & Above average & $3(23.1)$ & \\
\hline Motor & & 19 & Motor & & 27 \\
\hline Very low & $0(0)$ & & Very low & $5(62.5)$ & \\
\hline Low & $10(27.0)$ & & Low & $1(12.5)$ & \\
\hline Average & $21(56.8)$ & & Average & $1(12.5)$ & \\
\hline Above average & $6(16.2)$ & & Above average & $1(12.5)$ & \\
\hline Seizures (present) & $5(10.2)$ & 7 & Seizures (present) & $5(17.9)$ & 7 \\
\hline Neuropathy & & 9 & Neuropathy & & 8 \\
\hline Absent & $31(66.0)$ & & Stable & $23(85.2)$ & \\
\hline Mild & $14(29.8)$ & & Improved & $1(3.7)$ & \\
\hline Severe & $2(4.2)$ & & Worsened & $3(11.1)$ & \\
\hline Vision & & 7 & Vision & & 5 \\
\hline Low & $12(24.5)$ & & Stable & $23(76.7)$ & \\
\hline Blind & $2(4.1)$ & & Improved & $1(3.3)$ & \\
\hline Normal & $35(71.4)$ & & Worsened & $6(20.0)$ & \\
\hline Hearing & & 8 & Hearing & & 5 \\
\hline Mildly affected & $5(10.4)$ & & Stable & $27(90.0)$ & \\
\hline Severely affected & $2(4.2)$ & & Improved & $1(3.3)$ & \\
\hline Normal & $41(85.4)$ & & Worsened & $2(6.7)$ & \\
\hline School attendance* & & 5 & School attendance* & & 6 \\
\hline Regular & $35(79.5)$ & & Regular & $15(53.6)$ & \\
\hline Additional support & $7(15.9)$ & & Additional support & $9(32.1)$ & \\
\hline No attendance & $2(4.6)$ & & No attendance & $4(14.3)$ & \\
\hline School performance* & & 6 & School performance* & & 6 \\
\hline Appropriate for age & $33(82.5)$ & & Appropriate for age & $17(73.9)$ & \\
\hline Lower & $7(17.5)$ & & Lower & $6(26.1)$ & \\
\hline Independence & & 14 & Independence & & 10 \\
\hline Appropriate for age & $31(73.8)$ & & Appropriate for age & $16(64.0)$ & \\
\hline Needs assistance & $11(26.2)$ & & Needs assistance & $9(36.0)$ & \\
\hline Continence (incontinent) & $4(10.0)$ & 16 & Continence (incontinent). & $3(12.5)$ & 11 \\
\hline Adrenal insufficiency & & 32 & Adrenal insufficiency & & 8 \\
\hline Absent & $4(16.6)$ & & Stable & $25(92.6)$ & \\
\hline Glucocorticoids & $18(75)$ & & Worsened & $2(7.4)$ & \\
\hline Mineralcorticoids & $1(4.2)$ & & & & \\
\hline Combined & $1(4.2)$ & & & & \\
\hline
\end{tabular}

*only for pediatric patients 
or mild in 96\%) pre-CBT and remained stable in $89 \%$. Although hearing was rarely affected (96\% with normal or mild hearing loss) and remained stable or improved in 93\%, vision was affected more often. Normal vision was seen in $71 \%$ pre-CBT and worsened in $20 \%$ postCBT. Seizures were reported in $18 \%$ post-CBT. Almost all patients $(92.6 \%)$ experienced stable adrenal function. Data available on school performance showed that $74 \%$ had age appropriate school performance, educational support was provided to $32 \%$.

The most frequent interventions and complications after CBT were G-tube placement (13 with MLD, 20 patients with GLD, and 9 with X-ALD), followed by issues with dentition (9 with MLD, 14 with GLD, and 2 with X-ALD), and surgery (9 MLD, 9 GLD, and 2 X-ALD). Late pulmonary toxicity was seen in 16 patients ( 3 MLD, 4 GLD, and 9 X-ALD), and cardiac toxicity in 14 patients (5 MLD, 7 GLD, 2 X-ALD; supplemental table 2).

\section{DISCUSSION}

To the best of our knowledge, this retrospective study, spanning over 20 years of clinical experience, is the largest to describe both early and late outcomes after CBT in patients with leukodystrophies undergoing CBT. In this joint study of Eurocord, Inborn Errors WP-EBMT, and Duke University, we demonstrated a promising 1-year and 6-year OS (73\% and 61\%, respectively) and EFS (72\% and 59\%, respectively). Importantly, we observed an OS at 6 -years of nearly $80 \%$ in patients who were pre-symptomatic at time of CBT. Furthermore, the majority of these patients (53\%) maintained a performance score $>80$ after CBT at latest follow-up. This confirms previous smaller analyses ${ }^{6,19,22}$ that leukodystrophy patients transplanted prior to clinical symptoms experience long term survival while maintaining cognitive and motor function, compared to symptomatic or non-transplanted patients who all will deteriorate and/or die prematurely. Our results emphasize the importance of early diagnosis and treatment.

We observed rapid and robust neutrophil and platelet engraftment which supports other studies of CBT in LD. ${ }^{4,16}$ Time to neutrophil recovery was predicted by higher infused CD34+ cell dose and higher infused TNC as has been previously reported. ${ }^{4,15,16}$ In multivariate analysis of OS, patients who received grafts matched at 5-6/6 HLA loci or who were pre-symptomatic at time of CBT experienced improved OS. Conversely, those with poor performance status were at higher risk for morbidity (Supplemental Figure 3). While a recent report described $>95 \%$ of patients were alive and engrafted at 8 years after CBT for LSD in specialized centers ${ }^{17}$, these patients all met strict eligibility criteria and received harmonized conditioning regimens and GvHD-prophylaxis. It is important to acknowledge that our report reflects transplants 
that occurred in nearly 30 centers over a timeframe of almost 20 year. A not completely understood observation was the higher incidence of (mainly limited) chronic GvHD in the higher performance status group of patients. This may be due to very low number of survivors in the lowest performance group (only 9 of 29 survived). It is likely that refinements in patient eligibility, donor selection and improvements in supportive care that has occurred over the years will translate into improved outcomes in contemporary patient cohorts. ${ }^{18}$

It has been well-described that PS is an important predictor for survival and late outcomes after HCT, including CBT. ${ }^{5-8,19-22}$ Although PS is not the ideal tool for assessing neurocognitive development, this was the best available surrogate of cognitive function for long-term follow-up. It is important to note that, at most recent follow-up, there were some patients with discrepant PS and neurocognitive scores (i.e. PS $>80$, but a low or very low score on mental development or vice versa). Nevertheless, PS generally correlated with disease status and was able to quantify overall well-being that, even if less specific, can include general characteristics of the disease. Our results also demonstrated that there was minimal or no decline in PS beyond 1 year after CBT. Notably, pre-symptomatic disease was correlated with a higher probability of overall and event-free survival. Combining the PS before CBT and pre-symptomatic status could be used as a tool to predict outcomes. However, prospective studies that include longitudinal assessment of cognitive and motor function along with quality of life measures are warranted.

Moreover, this study emphasizes the importance of pre-symptomatic status and short duration from diagnosis to transplant on both early and late outcomes. These results provide further support that donor cord blood should be strongly considered when a non-carrier sibling donor is lacking. Due to the rapid course of these diseases, CB has some practical advantages above unrelated donors. While haplo-identical related donors are also readily available, most of these donors will be disease carriers. Previous studies of HCT in other LSDs have demonstrated an association between lower enzyme levels and worse late outcomes. ${ }^{23}$ Therefore, haploidentical donors are not routinely recommended in patients with leukodystrophies. Previous studies have also demonstrated that patients who achieved full donor chimerism experienced improved late outcomes. ${ }^{23,24}$ Although these studies were conducted in other LSDs, all studies with $\mathrm{CB}$ as donor source in LD showed high rates of full-donor chimerism. ${ }^{4-6}$ These results also support the use of newborn screening (NBS) which allows identification of neonates eligible for CBT at a time when they still experience maximal benefit. In select US and EU member states, NBS for GLD and/or X-ALD has been or will be implemented in upcoming years. ${ }^{6,25,26}$ To date, NBS for MLD is not available, but our results along with other studies provide evidence that early diagnosis and transplant prior to onset of symptoms improve early and late outcomes after CBT. ${ }^{8,9,21,27}$ 
This study recognizes that the long-term outcomes of many children are affected to various extents by their underlying disease, the transplant procedure or a combination of both. While nearly half of the children in this study were able to perform at an age appropriate level at school, many needed additional support services to achieve that level of performance, especially MLD and GLD patients. Although these results are better than the natural course in non-transplanted patients, ${ }^{28,29}$ more research concerning the effects of transplantation on functional outcomes and quality-of-life is warranted to optimize late outcomes including the quality-of-life of these patients.

Further research is also needed to better understand the duration for which CBT can provide a therapeutic effect. It remains unclear whether the cross-correction of the deficient enzyme after CBT completely halts disease in MLD and GLD as significant post-transplantation peripheral nerve disease has been reported by others. ${ }^{6,8}$ Lentiviral haemopoietic stem cell gene therapy (HSC-GT) that aims for supra-normal enzyme-levels, may provide a solution for this in the future, as a recent report suggests for MLD patients. Although promising, longer follow-up is needed and only half of patients achieved supra-normal enzyme levels. ${ }^{30}$

In conclusion, $\mathrm{CBT}$, in patients with LD showed promising early and late outcomes, especially for those who are pre-symptomatic before CBT and those who received adequately dosed grafts. Early identification and treatment appears to be an important predictor of outcomes, which suggests that NBS may further improve outcomes. Furthermore, fast referral to a specialized center after diagnosis is necessary. For symptomatic patients transplanted with a low performance score $(<60$ and $60-80)$, only $5 \%$ and $12 \%$, respectively, of these patients were alive with improved performance at last known follow-up. Counseling of these patients and parents is of extreme importance. As life expectancy for patients with LSDs increases after CBT, international collaboration is of utmost importance to further optimize therapies, including CBT, to improve outcomes for these patients. Moreover, prospective trials comparing new (gene) therapies to the standard therapies are needed.

\section{ACKNOWLEDGEMENTS}

The authors thank the collaborating transplant centres for sharing patient information: Hospital La Timone, Marseille, France; King Faisal Specialist Hospital \& Research Centre, Riyadh, Saudi Arabia; Niño Jesus Children's Hospital, Madrid, Spain; University Hospital Gent, Gent, Belgium; Randwick Sydney Children's Hospital, Sydney, Australia; The Children's Hospital at Westmead, Sydney, Australia; Necker Hospital, Paris, France; Cliniques Universitaires St. Luc, Brussels, Belgium; Monza Ospedale San Gerardo Clinica Pediatrica dell'Università 
di Milano Bicocca, Italy; Hospital Clínico Universitario Virgen de la Arrixaca, Murcia, Spain; Hospital Vall d'Hebron, Barcelona, Spain; CHU Sainte-Justine, Montréal, Canada; Universitair Ziekenhuis Brussel, Brussels, Belgium; St. László Hospital, Budapest, Hungary; University Medical Center, Leiden, The Netherlands; University Hospital GasthuisbergUniversity of Leuven, Leuven, Belgium; Hadassah University Hospital, Jerusalem, Israel; Pitié-Salpêtrière Hospital, Paris, France; Instituto Português De Oncologia do Porto Francisco Gentil, Porto, Portugal; Medical School Dept. Haematology/Oncology/Stem Cell Transplantation, Hannover, Germany; Instituto Português De Oncologia de Lisboa Francisco Gentil, Lisboa, Portugal; Charité-CVK University Medicine, Berlin, Germany; Rambam Medical Center. Haifa, Israel; Department of Paediatric Haematology, Manchester, United Kingdom; Akdeniz University Medical School, Antalya, Turkey; University of Medical Sciences Poznan, Poznan, Poland; CHU Brabois, Nancy, France; Children's University Hospital, Bratislava, Slovakia; Wilhelmina Children's Hospital, Utrecht, The Netherlands; and, Duke University Medical Center, Durham, United States of America.

Furthermore, the authors would like to thank Mahsa Taskindoust for helping with the data collection at Duke University Medical Center. A complete list of the members of the Eurocord, Inborn Errors Working Party of European Society for Blood and Marrow Transplantation and Duke University appears in "Appendix: study group members." 


\section{REFERENCES}

1. Boelens J, van Hasselt P. Neurodevelopmental Outcome after Hematopoietic Cell Transplantation in Inborn Errors of Metabolism: Current Considerations and Future Perspectives. Neuropediatrics. 2016; 47: 285-292.

2. Aldenhoven M, Kurtzberg J. Cord blood is the optimal graft source for the treatment of pediatric patients with lysosomal storage diseases: clinical outcomes and future directions. Cytotherapy. 2015; 17: 765-774.

3. Wynn R. Stem cell transplantation in inherited metabolic disorders. Hematol Am Soc Hematol Educ Progr. 2011; 2011: 285-291.

4. Martin PL, Carter SL, Kernan NA, et al. Results of the cord blood transplantation study (COBLT): outcomes of unrelated donor umbilical cord blood transplantation in pediatric patients with lysosomal and peroxisomal storage diseases. Biol Blood Marrow Transplant. 2006; 12: 184-194.

5. Prasad VK, Mendizabal A, Parikh SH, et al. Unrelated donor umbilical cord blood transplantation for inherited metabolic disorders in 159 pediatric patients from a single center: influence of cellular composition of the graft on transplantation outcomes. Blood. 2008; 112: 2979-2989.

6. Escolar ML, Poe MD, Provenzale JM, et al. Transplantation of umbilical-cord blood in babies with infantile Krabbe's disease. N Engl J Med. 2005; 352: 2069-2081.

7. Beam D, Poe MD, Provenzale JM, et al. Outcomes of unrelated umbilical cord blood transplantation for X-linked adrenoleukodystrophy. Biol Blood Marrow Transplant. 2007; 13: 665-674.

8. Martin HR, Poe MD, Provenzale JM, Kurtzberg J, Mendizabal A, Escolar ML. Neurodevelopmental outcomes of umbilical cord blood transplantation in metachromatic leukodystrophy. Biol Blood Marrow Transplant. 2013; 19: 616-624.

9. Groeschel S, Kühl J-S, Bley AE, et al. Long-term Outcome of Allogeneic Hematopoietic Stem Cell Transplantation in Patients With Juvenile Metachromatic Leukodystrophy Compared With Nontransplanted Control Patients. JAMA Neurol. 2016: 1-8.

10. Escolar ML, Poe MD, Martin HR, Kurtzberg J. A Staging System for Infantile Krabbe Disease to Predict Outcome After Unrelated Umbilical Cord Blood Transplantation. Pediatrics. 2006; 118: 879-889.

11. Boelens JJ, Aldenhoven M, Purtill D, et al. Outcomes of transplantation using various hematopoietic cell sources in children with Hurler syndrome after myeloablative conditioning. Blood. 2013; 121).

12. Glucksberg H, Storb R, Fefer A, et al. Clinical manifestations of graft-versus- 
host disease in human recipients of marrow from HL-A-matched sibling donors. Transplantation. 1974; 18: 295-304.

13. Shulman HM, Sullivan KM, Weiden PL, et al. Chronic graft-versus-host syndrome in man. A long-term clinicopathologic study of 20 Seattle patients. Am J Med. 1980; 69: 204-217.

14. van Rappard DF, Bugiani M, Boelens JJ, et al. Gallbladder and the risk of polyps and carcinoma in metachromatic leukodystrophy. Neurology. 2016; 87: 103-111.

15. Kim J, Sun Z, Ezekian B, et al. Gallbladder abnormalities in children with metachromatic leukodystrophy. J Surg Res. 2017; 208: 187-191.

16. Boelens JJ, Rocha V, Aldenhoven M, et al. Risk factor analysis of outcomes after unrelated cord blood transplantation in patients with hurler syndrome. Biol Blood Marrow Transplant. 2009; 15: 618-625.

17. Aldenhoven M, Jones SA, Bonney D, et al. Hematopoietic Cell Transplantation for Mucopolysaccharidosis Patients Is Safe and Effective: Results after Implementation of International Guidelines. Biol Blood Marrow Transplant. 2015; 21: 1106-1109.

18. Lum SH, Miller WP, Jones S, et al. Changes in the incidence, patterns and outcomes of graft failure following hematopoietic stem cell transplantation for Hurler syndrome. Bone Marrow Transplant. February 2017.

19. Miller WP, Rothman SM, Nascene D, et al. Outcomes after allogeneic hematopoietic cell transplantation for childhood cerebral adrenoleukodystrophy: the largest singleinstitution cohort report. Blood. 2011; 118: 1971-1978.

20. Musolino PL, Lund TC, Pan J, et al. Hematopoietic stem cell transplantation in the leukodystrophies: a systematic review of the literature. Neuropediatrics. 2014; 45: 169-174.

21. Boucher AA, Miller W, Shanley R, et al. Long-term outcomes after allogeneic hematopoietic stem cell transplantation for metachromatic leukodystrophy: the largest single-institution cohort report. Orphanet J Rare Dis. 2015; 10:94.

22. van Rappard DF, Boelens JJ, van Egmond ME, et al. Efficacy of hematopoietic cell transplantation in metachromatic leukodystrophy: the Dutch experience. Blood. 2016; 127: 3098-3101.

23. Aldenhoven M, Wynn RF, Orchard PJ, et al. Long-term outcome of Hurler syndrome patients after hematopoietic cell transplantation: an international multicenter study. Blood. 2015; 125: 2164-2172.

24. Lum SH, Ghosh A, Stepien K, et al. Long term survival and cardiopulmonary outcome in children with Hurler syndrome after haematopoietic stem cell transplantation in Manchester. Mol Genet Metab. 2017; 12: 87-88.

25. Msall M, Duffner P, Lyon N, Shapiro E. Health, developmental, and functional 
outcome surveillance in preschool children with lysosomal storage diseases (LSD). Mol Genet Metab. 2009; 96: 32.

26. Wasserstein MP, Andriola M, Arnold G, et al. Clinical outcomes of children with abnormal newborn screening results for Krabbe disease in New York State. Genet Med. 2016; 18: 1-9.

27. Allewelt HB, Page K, Taskindoust M, et al. Long-Term Functional Outcomes following Hematopoietic Stem Cell Transplantation for Krabbe Disease. Biol Blood Marrow Transplant. 2016; 22: 102-103.

28. Krägeloh-Mann I, Groeschel S, Kehrer C, et al. Juvenile metachromatic leukodystrophy 10 years post transplant compared with a non-transplanted cohort. Bone Marrow Transplant. 2013; 48: 369-375.

29. Bonkowsky JL, Nelson C, Kingston JL, Filloux FM, Mundorff MB, Srivastava R. The burden of inherited leukodystrophies in children. Neurology. 2010; 75: 718-725.

30. Sessa M, Lorioli L, Fumagalli F, et al. Lentiviral haemopoietic stem-cell gene therapy in early-onset metachromatic leukodystrophy: an ad-hoc analysis of a nonrandomised, open-label, phase 1/2 trial. Lancet. 2016; 388: 476-487. 


\section{SUPPLEMENTAL FIGURES}
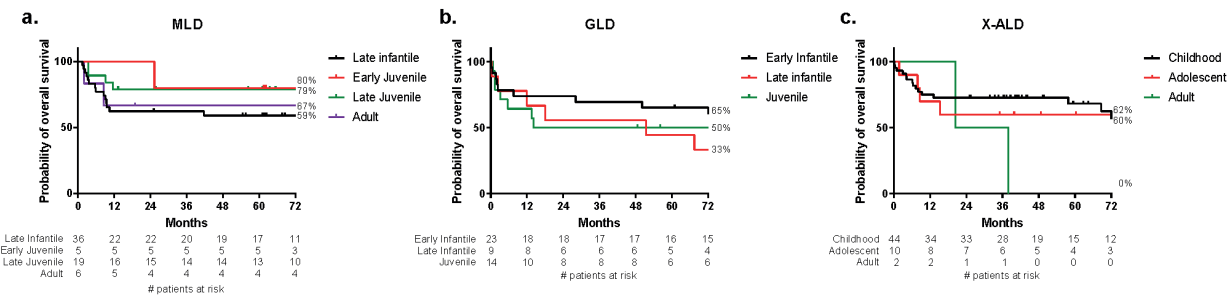

Suppl. figure 1. 6-Year overall and event-free survival per disease and categorized per clinical phenotype. a: MLD; b: GLD; c: X-ALD. Events were considered to be autologous reconstitution, graft failure or death.

a

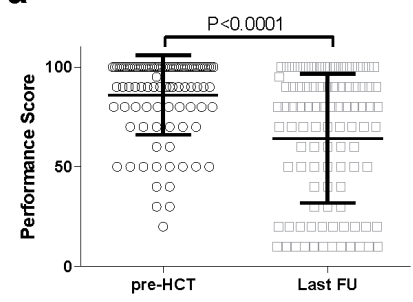

C

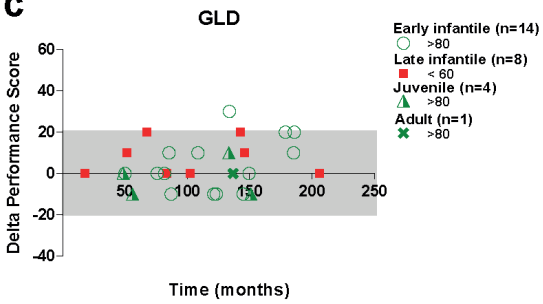

b
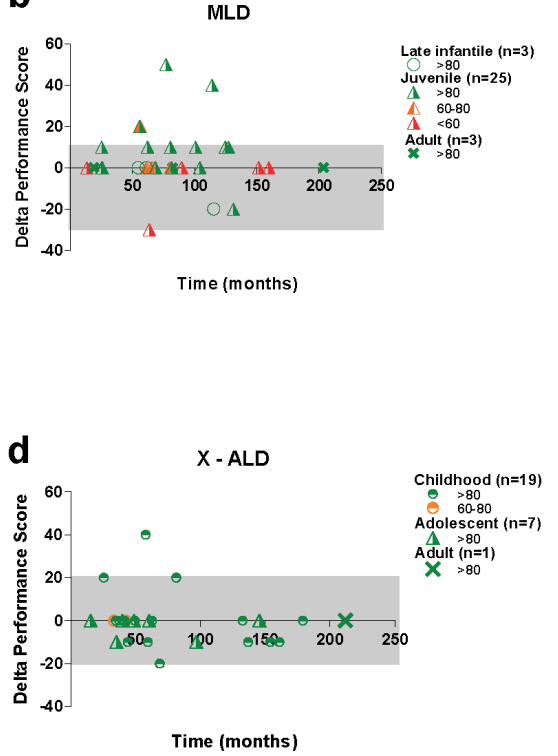

Suppl. figure 2. Evolution of performance score before and after CBT. (a) Individual PS of patients with $\geq 12$ months survival before and at last follow-up after CBT; (b)(c)(d) Delta of PS at 12 months and at last follow-up per disease and categorized per clinical phenotype; a positive delta is associated with decline in PS. (b: MLD; c: GLD; d: X-ALD). Legends provide color coding for pre-CBT performance score and pictograms for different clinical phenotypes). 
Overall

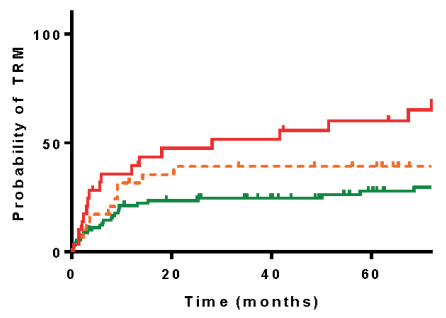

GLD

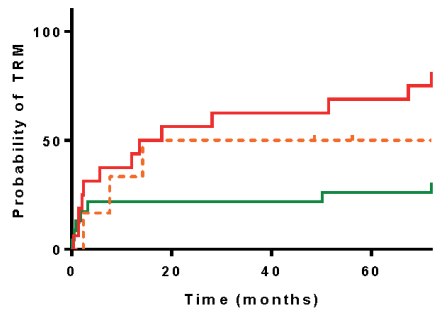

M LD

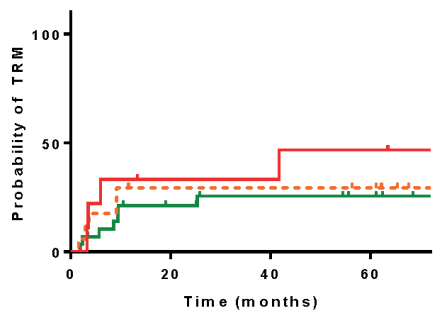

$$
\begin{aligned}
& \text { - Pre-CBT PS }<60 \\
& \text {-L. Pre-CBT PS } 60-80 \\
& \text { - Pre-CBT PS }>80
\end{aligned}
$$

$X-A L D$

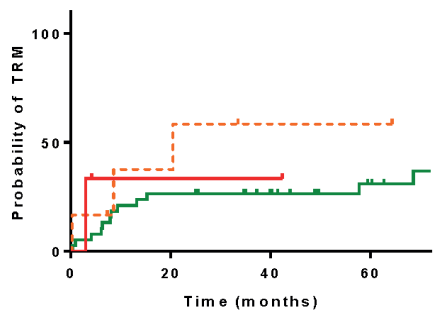

Suppl. figure 3. Probabilities of transplant related mortality by performance score at time of transplantation. 


\section{SUPPLEMENTAL TABLES}

Suppl. table 1. Causes of death.

\begin{tabular}{lcccc}
\hline & Overall & MLD & GLD & X-ALD \\
& $\mathbf{n}(\%)$ & n (\%) & n (\%) & $21(38)$ \\
\hline Overall & $69(41)$ & $23(35)$ & $25(38)$ & $16(76)$ \\
Transplant-related & $43(25)$ & $13(57)$ & $14(56)$ & 2 \\
Viral infection & 8 & 3 & 3 & 2 \\
Bacterial infection & 7 & 2 & 3 & 1 \\
Fungal infection & 3 & 0 & 2 & 3 \\
GvHD & 9 & 3 & 3 & 1 \\
MOF & 5 & 3 & 1 & 0 \\
Cardiac toxicity & 1 & 1 & 1 & 4 \\
Pulmonary disease & 5 & 0 & 1 & 1 \\
VOD & 2 & 0 & 0 & 1 \\
Pancytopenia & 1 & 0 & 0 & 1 \\
Unknown & 1 & 0 & $7(28)$ & $5(24)$ \\
Disease progression & $21(31)$ & $9(39)$ & $4(16)$ & 0 \\
Unknown & $5(7)$ & $1(4)$ & & \\
\hline
\end{tabular}

MOF, multi organ failure; VOD, veno occlusive disease

Suppl. table 2. Complications.

\begin{tabular}{|c|c|c|c|c|c|c|c|c|c|c|c|c|}
\hline \multirow{2}{*}{ Complication } & \multicolumn{3}{|c|}{ Overall } & \multicolumn{3}{|c|}{ MLD (66) } & \multicolumn{3}{|c|}{ GLD (47) } & \multicolumn{3}{|c|}{ X-ALD (56) } \\
\hline & D & $\mathbf{A}$ & $\mathbf{T}$ & D & $\mathbf{A}$ & $\mathbf{T}$ & D & $\mathbf{A}$ & $\mathbf{T}$ & D & $\mathbf{A}$ & $\mathbf{T}$ \\
\hline$G(/ J)$-tube placement and/or gastrostomy & 22 & 17 & 39 & 4 & 9 & 13 & 12 & 8 & 20 & 8 & 1 & 9 \\
\hline Pulmonary toxicity & 11 & 5 & 16 & 1 & 2 & 3 & 3 & 1 & 4 & 7 & 2 & 9 \\
\hline Cardiac toxicity & 10 & 4 & 14 & 4 & 1 & 5 & 4 & 3 & 7 & 2 & - & 2 \\
\hline Dentition & 4 & 17 & 21 & 4 & 5 & 9 & 4 & 10 & 14 & - & 2 & 2 \\
\hline Surgery & 6 & 14 & 20 & 3 & 6 & 9 & 3 & 6 & 9 & - & 2 & 2 \\
\hline Cholecystectomy & 1 & 3 & 4 & 1 & 3 & 4 & - & - & - & - & - & - \\
\hline $\begin{array}{l}\text { Tendon release/ } \\
\text { bone surgery }\end{array}$ & - & 8 & 8 & - & 3 & 3 & - & 5 & 5 & - & - & - \\
\hline VP-shunt & 3 & - & 3 & - & - & - & 3 & - & 3 & - & - & - \\
\hline Other* & 2 & 3 & 5 & 2 & - & 2 & - & 1 & 1 & - & 2 & 2 \\
\hline
\end{tabular}

D, Death; A, Alive; T, Total

*Including hemicolectomy/colostomy/ileostomy, removal of nasal lesion, vagal nerve stimulator 
Outcomes of CBT in Inherited Leukodystrophies $\mid 45$ 


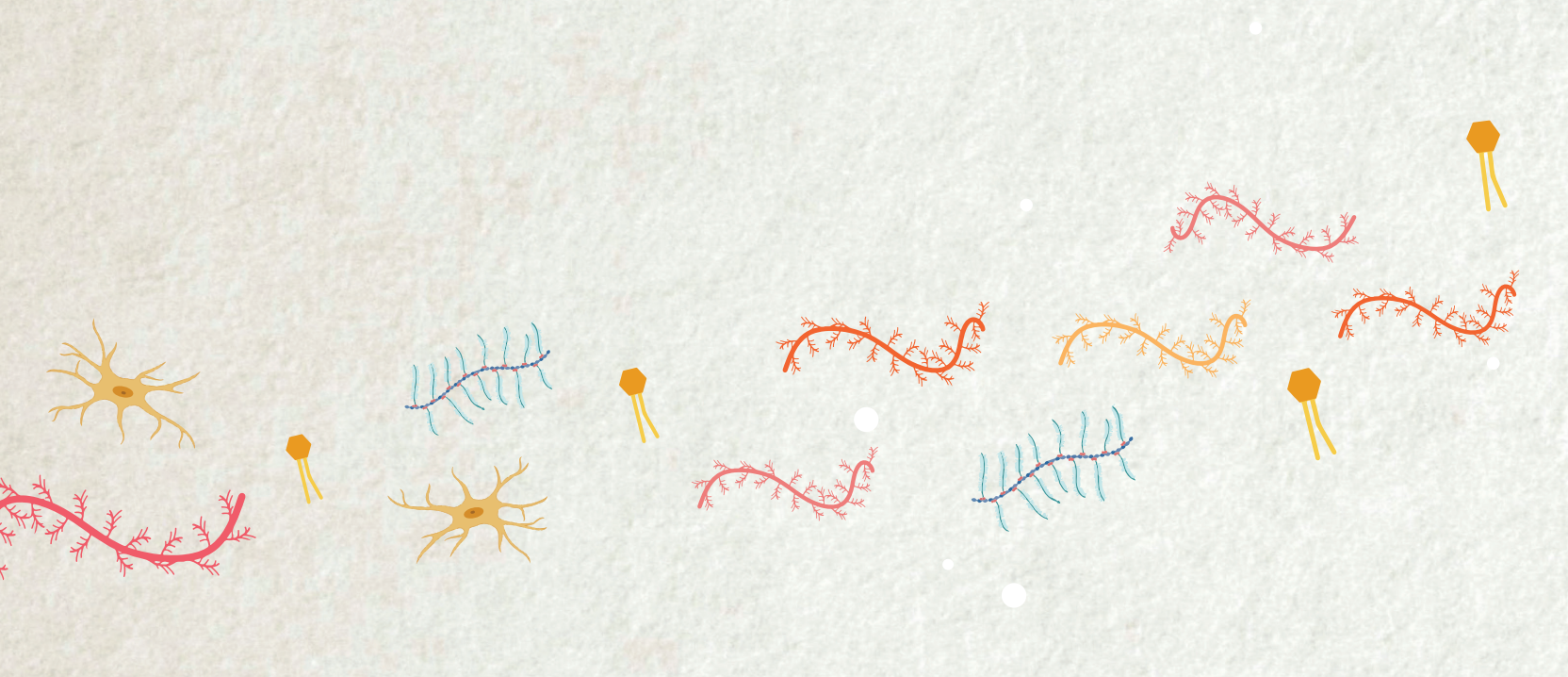



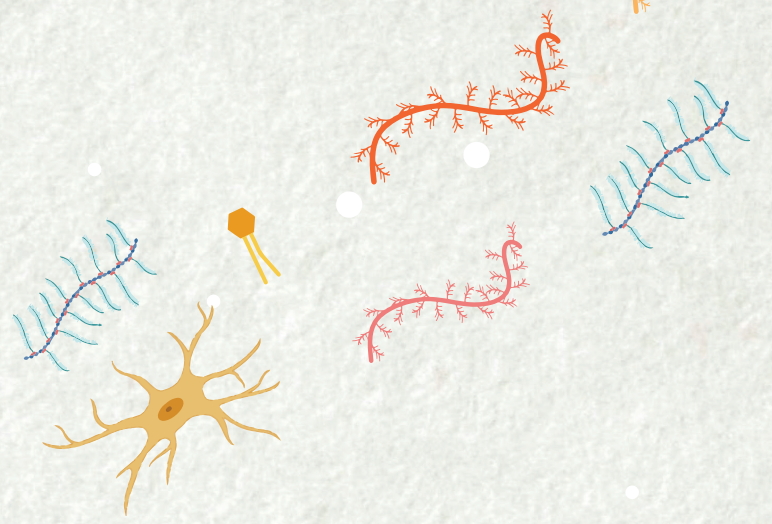

\section{CHAPTER 3}

\section{Longitudinal Analysis of Ocular Disease in Children with Mucopolysaccharidosis I after Hematopoietic Cell Transplantation}

Brigitte T.A. van den Broek

Michelle B. van Egmond-Ebbeling

Jens A. Achterberg

Jaap Jan Boelens

Isa C. Vlessert

Hubertus C.M.T. Prinsen

Jaap van Doorn

Peter M. van Hasselt 


\section{ABSTRACT}

Aim: Corneal clouding, causing visual impairment, is seen in nearly all patients with Mucopolysaccharidosis type 1 (MPS-1). Hematopoietic cell transplantation (HCT) is able to stabilize disease in many organs. Residual disease in several tissues however is increasingly recognized. Data on the effect of HCT on ocular disease in patients with MPS-1 are contradictory. With this study we aim to clarify the long-term effect of HCT on ocular disease in these patients.

Methods: Best Corrected Visual Acuity (BCVA), refraction, intra-ocular pressure (IOP), slit-lamp biomicroscopic and fundoscopic examinations including corneal clouding were prospectively collected from MPS-1 patients treated with HCT between 2003 and 2018 . The course of corneal clouding and BCVA after HCT were analyzed using a linear mixed model. Other parameters studied were clinical phenotype, age at time of transplantation and hematological enzyme activity after transplantation. Outcomes of additional ophthalmologic tests were described. Additionally, IDUA and AGAL enzyme activities and GAGs concentration in tear fluid were determined.

Results: 24 engrafted MPS- 1 patients were included (92\% with $>95 \%$ chimerism and normal enzyme activity after HCT). Corneal clouding stabilized first years after HCT, but increased rapidly beyond three years $(p<0.0001)$. BCVA and IOP also worsened over time $(p=0.01$ and $\mathrm{p}<0.0001$, respectively). IDUA activity in tear fluid remained very low in patients $(\mathrm{p}<0.0001)$.

Conclusion: After initial stabilization in the cornea, ongoing ocular disease and low IDUA activity in tear fluid is seen in MPS-1 patients despite treatment with HCT, unveiling a weak spot of current standard therapy. New therapies that overcome these shortcomings are necessary to improve the late outcomes of patients. 


\section{INTRODUCTION}

Corneal clouding is a hallmark of Mucopolysaccharidoses. ${ }^{1-3}$ The incidence in Mucopolysaccharidosis-1 (MPS-1) is $97.6-100 \%$ and causes visual impairment which is a burden on these patients. ${ }^{4,5}$ MPS- 1 is an autosomal recessive inherited metabolism disorder characterized by the accumulation of glycosaminoglycans (GAGs) causing multi-systemic disease. Nearly 300 mutations in the $\alpha$-L-iduronidase (IDUA) gene are known. Pathogenic mutations affect the availability and enzymatic activity of the lysosomal enzyme IDUA and result in a phenotypic spectrum ranging from the severe Hurler to the milder Scheie syndrome. Before the advent of hematopoietic cell transplantation (HCT) and enzyme replacement therapy, severe MPS-1 patients had a median life expectancy of 5 years, only rarely surviving beyond 10 years of age. HCT has resulted in a significant longer life expectancy (3- to 5-year overall survival of 74 to $95 \%$ ) and a reduction in the overall severity of systemic disease manifestations. ${ }^{6-10}$ Initial improvement of ocular findings are described after HCT, including improvement of corneal clouding. ${ }^{1,5}$ The hematological enzyme level after HCT as well as the age at time of transplantation and clinical phenotype have shown to be predictors for improved clinical outcomes, including ocular manifestations. ${ }^{5}$ However, although patients are being transplanted as early as possible and transplant protocols have been optimized resulting in normal hematological enzyme activity in most patients, residual disease remains substantial and diminishes quality of life. ${ }^{5,7,11}$ Whether this is also true for ocular manifestations remains uncertain as previous studies have shown both improvement and worsening of the ocular phenotype in patients with MPS- 1 after HCT. ${ }^{1,5,12}$ Routine followup over a time span of more than 10 years at the 'Sylvia Toth Center for Multidisciplinary Follow-up after Hematopoietic Cell Transplantation' in the Wilhelmina Children's Hospital, Utrecht, The Netherlands, allowed us to elucidate this question. In this study we describe a longitudinal analysis of the long-term effect of HCT on ocular manifestations from a unique cohort of MPS-1 patients.

Normally, there are no blood vessels in the cornea. The oxygen supply to this tissue takes place through gas exchange with the atmosphere. MPS-1 patients who have undergone HCT may therefore be designated for IDUA supply to the cornea from another source, probably tear fluid. The removal of excess, accumulated GAGs from the cornea may also go through the tear fluid. Therefore it is important to get more insight in the presence of GAGs and the penetration of donor derived IDUA into tear fluid of transplanted patients. Hence, we analyzed the specific activities of IDUA and $\alpha$-galactosidase A (AGAL), and the concentrations of heparan sulphate (HS), dermatan sulphate (DS), and keratan sulphate (KS) in tear fluid of both MPS-1 patients after HCT and healthy controls. 


\section{PATIENTS AND METHODS}

\section{Data collection}

All MPS-1 patients successfully treated with HCT in the Wilhelmina Children's Hospital (WKZ) of the University Medical Centre Utrecht (UMCU), The Netherlands between 2003 and 2018, were included in this study and evaluated by an experienced ophthalmologist on a yearly basis at the 'Sylvia Toth Center for Multidisciplinary Follow-up after Hematopoietic Cell Transplantation'. The MPS-1 phenotype (Hurler or Hurler-Scheie), age at transplantation, IDUA enzyme activity level after transplantation (scored as below or above lower limit of normal, cut-off point: $20 \mathrm{nmol} / \mathrm{h} / \mathrm{mg}$ ), grade of corneal clouding (as documented by the clinical assessment score: 0 , no corneal haze; 1 , iris details visible; 2, iris details not visible but pupillary margins visible; 3, pupillary margin not visible; 4, total opacity), LogMar best corrected visual acuity (BCVA), refractive error (cycloplegic retinoscopy or autorefraction with the TopCon KR-1 [TopCon Medical Systems, Oakland, $\mathrm{NJ}$ ), orthoptic examinations, intra-ocular pressure (Icare PRO [Icare Finland, Helsinki, Finland]), and slit-lamp biomicroscopic and fundoscopic examinations were collected. Other additional ophthalmologic parameters, including, visual fields (Rodenstock Peritest), optical coherence tomography (OCT), axial length, corneal thickness, electroretinography (ERG) and visual evoked potentials (VEP) were collected on indication. Data from healthy children on refractive error, axial length, corneal thickness, and IOP were extracted from recent published papers. ${ }^{13-16}$ Part of this cohort has been reported in previous studies. ${ }^{17}$ This study was performed in agreement with the Helsinki Declaration of 1975, revised in 2008. This study is based on patient data acquired during care-as-usual and has been exempted for approval according to the responsible institutional committee Medical Research Involving Human Subjects Act.

\section{Collection of tear fluid}

Tear fluid from MPS-1 patients and healthy controls were collected with the use of Schirmer strips. ${ }^{18}$ To eluate the tear fluid, the Schirmer strips were cut into pieces of $5 \mathrm{~mm}$ and placed in a $1 \mathrm{ml}$ Eppendorf tube. Next, $300 \mu \mathrm{l}$ reverse osmosis (RO) water was added and the tubes were placed in an Eppendorf thermoshaker and gently shaken (500 rpm) for one hour at room temperature (RT). Samples were subsequently centrifuged at $10.000 \mathrm{x}$ g for $5 \mathrm{~min}$ at $4^{\circ} \mathrm{C}$. The eluates of both eyes were pooled and $50 \mu \mathrm{l}$ was used for the measurement of protein content, $100-200 \mu \mathrm{l}$ for GAG content and $260 \mu \mathrm{l}$ was used for analysis of IDUA and AGAL enzyme activity. Samples for analysis of the enzyme activity were diluted 1:1 with 5\% BSA solution to stabilize the activity of lysosomal enzymes, and if not directly measured, stored at $-80^{\circ} \mathrm{C}$ until analysis for a maximum of three days. 


\section{Determination of IDUA and AGAL enzyme activity and HS, DS, and KS concentrations in tear fluid}

The measurements of IDUA (Wallac 1420-050 Multilabel Counter; Perkin Elmer, Turku, Finland), and, as a control, AGAL (EC 3.2.1.22) activities in tear fluid were performed in principle according to procedures described by Chamoles et al. for dried blood spots and modified previously by us for application to saliva. ${ }^{17,19}$ The specificities of the IDUA and AGAL assays in samples of tear fluid were validated after immune precipitation with sheep anti-hIDUA antibody (R\&D systems, Europe Ltd., Abingdon, UK) using protein G coupled Dynabeads $^{\mathrm{TM}}$ (Invitrogen, ThermoFisher, Landsmeer, the Netherlands), and in the presence and absence of the specific AGAL inhibitor, 1-deoxygalactonojirimycin hydrochloride, respectively (data not shown).17 For both IDUA and AGAL, the results were calculated from the 4-methylumbelliferyl (4-MU) calibration curve and expressed as nmol 4MU/hr.mg protein. Protein contents of the various tear fluid samples were determined by the Pierce TM bicinchoninic acid (BCA) assay (Thermo Fisher Scientific, Rockford,IL,USA).

HS, DS, and KS were determined according to the method described by Langereis et al. ${ }^{20}$ with slight modifications. Tear fluid eluates of $100-200 \mu$ l were concentrated by evaporating the matrix using nitrogen at $40^{\circ} \mathrm{C}$ and samples were resolved in Milli-Q water. $\Delta \mathrm{UA}-2 \mathrm{~S}{ }^{\circledR}$ GlcNCOEt-6S (I-P) (Iduron, Alderley Edge, United Kingdom) was used as internal standard. Enzymes Heparinase II ( $5 \mathrm{mIU} / \mathrm{sample})$, Keratinase II $(0.3 \mu \mathrm{l} / \mathrm{sample})$ and Chondroitinase B (50 mIU/sample) were obtained from Academic Medical Centre Amsterdam (in-house manufactured) and were used to digest HS, KS and DS to GAG-derived disaccharides. The GAG-derived disaccharides were ionized by Electrospray in negative mode and separated

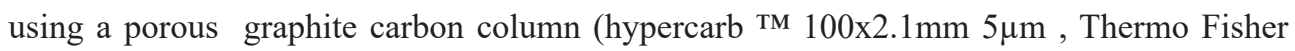
Scientific, Waltham, USA) and analyzed by LC-MS-MS (Waters, Etten-Leur, the Netherlands). The mobile phase consisted of $10 \mathrm{mM}$ ammoniumformiate $\mathrm{pH} 10$ and $100 \%$ acetonitril. Ammonium formiate and acetonitrile were purchased from Biosolve (Valkenswaard, the Netherlands).

\section{Statistical analysis}

Baseline characteristics are reported as frequencies and percentages for categorical variables and median (range) for continuous variables. To analyze the course of corneal clouding, BCVA, refractive error, and IOP over time after transplantation a linear mixed model was fitted. To account for non-linearity, restricted cubic splines with three knots were added to the model. Based on previous literature, age at time of transplantation, phenotype, and enzyme level after transplantation were considered important covariates and included as fixed effects. A random intercept was included per individual to account for individual variation at baseline and a random slope (for time) for dependency across the repeated measurements within the 
same individual during follow-up. To account for dependency between both eyes in the analysis for BCVA a random intercept per eye was also included. For corneal clouding, IOP and refraction, only data of the right eye was used for the analysis as there was a nearly perfect correlation between the left and right eye. Final coefficients were estimated using restricted maximum likelihood and significance of parameters was assessed with a likelihood ratio test between the model with and without the parameter. The model assumptions including normal distributed residuals, random effects, and homogeneity of variance were confirmed visually. Correlation between corneal clouding and BCVA was tested by Pearson's Rho. Finally, differences between enzyme activities and HS, DS, and KS concentrations between patients and controls were analyzed using the Student $t$ test, and the Bonferroni-Sidak method was applied to correct for multiple testing. P-values $<0.05$ were considered significant. The $\mathrm{R}$ project (RStudio: Integrated Development for R. RStudio, Inc., Boston, MA) for statistical computing version 3.4.1 with the packages ' $1 \mathrm{me} 4$ ', 'rms', and 'ggplot2' and GraphPad Prism (GraphPad Prism version 7.00 for Windows, GraphPad Software, La Jolla California USA) were used for all analyses and for the creation of figures.

\section{RESULTS}

The analysis included 170 observations of twenty-four transplanted patients. The majority of the patients $(92 \%)$ had the severe Hurler phenotype. Median age at transplantation was 1.17 years (range 0.23-2.35) for Hurler patients and 2.12 years (range 1.23-3.01) for Hurler-Scheie patients. Of all patients after HCT, 92\% had normal IDUA enzyme activities in leucocytes (range $20-90 \mathrm{nmol} / \mathrm{h} / \mathrm{mg}$ ). Median follow-up was 8.0 years (0.6-14.3). At baseline, $91 \%$ had absent to mild corneal clouding. See table 1 for patient characteristics.

\section{Corneal clouding}

Figure 1 visualizes corneal clouding over time as estimated by a mixed model for the 'average' patient, which is based on individual patient data (shown faintly in the background). The solid blue line describes the degree of corneal clouding over time representative for a patient with the Hurler phenotype, mean age at transplantation (1.17 years) and IDUA enzyme activities in the normal range. The dashed blue line describes the estimated course characteristic for a Hurler-Scheie patient, with the mean age at transplantation (2.01 years) and IDUA enzyme activities in the normal range. Corneal clouding is stable during the first three years after transplantation, but thereafter increases severely $(\mathrm{p}<0.0001)$. This deterioration seems to set in sooner for Hurler patients than Hurler-Scheie patients. Overall, clouding is significantly more severe in Hurler patients compared to Hurler-Scheie patients (table 2; p $=0.01$ ). Furthermore, age at time of transplantation was a significant risk factor for corneal 
clouding (table $2 ; \mathrm{p}=0.0003$ ). The level of IDUA enzyme activity measured in leukocytes post transplantation was not found to be a significant risk factor (table $2 ; \mathrm{p}=0.48$ ). Together, these findings imply that treatment with HCT may slower disease progression in the cornea, but fails to halt it.

Table 1. Patient characteristics $(n=24)$

\begin{tabular}{lc}
\hline Patient characteristic & $\mathbf{n}(\mathbf{\%})$ \\
\hline Sex (male) & $15(63 \%)$ \\
Phenotype (severe) & $22(92 \%)$ \\
Age at transplantation (years; median, range) & $1.22(0.23-3.01)$ \\
$\quad$ Hurler & $1.17(0.23-2.35)$ \\
$\quad$ Hurler-Scheie & $2.12(1.23-3.01)$ \\
Donor source (CB/BM) & $18(75 \%) / 6(25 \%)$ \\
Enzyme level post transplantation (>LLN) & $22(92 \%)$ \\
Chimerism (>95\%) & $22(92 \%)$ \\
Follow-up in years (median, range) & $8.0(0.6-14.3)$ \\
Baseline cornea clouding OD/OS & \\
$\quad$ 0: no corneal haze & $3(13 \%) / 4(17 \%)$ \\
$\quad$ 1: iris details visible & $14(61 \%) / 13(57 \%)$ \\
2: iris details not visible but pupillary & $4(17 \%) / 4(17 \%)$ \\
$\quad$ margins visible & \\
3: pupillary margins not visible & \\
4: total opacity & $0(0 \%) / 0(0 \%)$ \\
\hline CB, cord blood; BM, bone marrow; LLN, lower limit of normal; OD, oculus dexter; OS, oculus \\
sinister
\end{tabular}

\section{Visual acuity}

Best corrected visual acuity (BCVA) is also worsening over the years ( $p=0.01$; fig. 2). Contrary to corneal clouding, the decline appears more gradually. The blue line depicts visual acuity for the average Hurler patient with mean age at transplantation (1.17 years) and IDUA enzyme activities in the normal range. Hurler-Scheie patients have better visual acuity (table $2 ; p=0.04$; dashed line figure 2). Visual acuity was not found to be better for both lower age at time of transplantation and IDUA enzyme activity after transplantation within the normal range (table $2 ; \mathrm{p}=0.22$ and $\mathrm{p}=0.32$, respectively). Visual acuity was strongly inversely correlated with corneal clouding for both eyes (OD: $\rho=-.63$, $p$-value $=7.12 \mathrm{e}^{-11}$; OS: $\rho=-.55$, $\mathrm{p}$-value $=3.21 \mathrm{e}^{-9}$, figure 3 ), indicating that decreasing visual acuity can be largely explained by corneal clouding. 


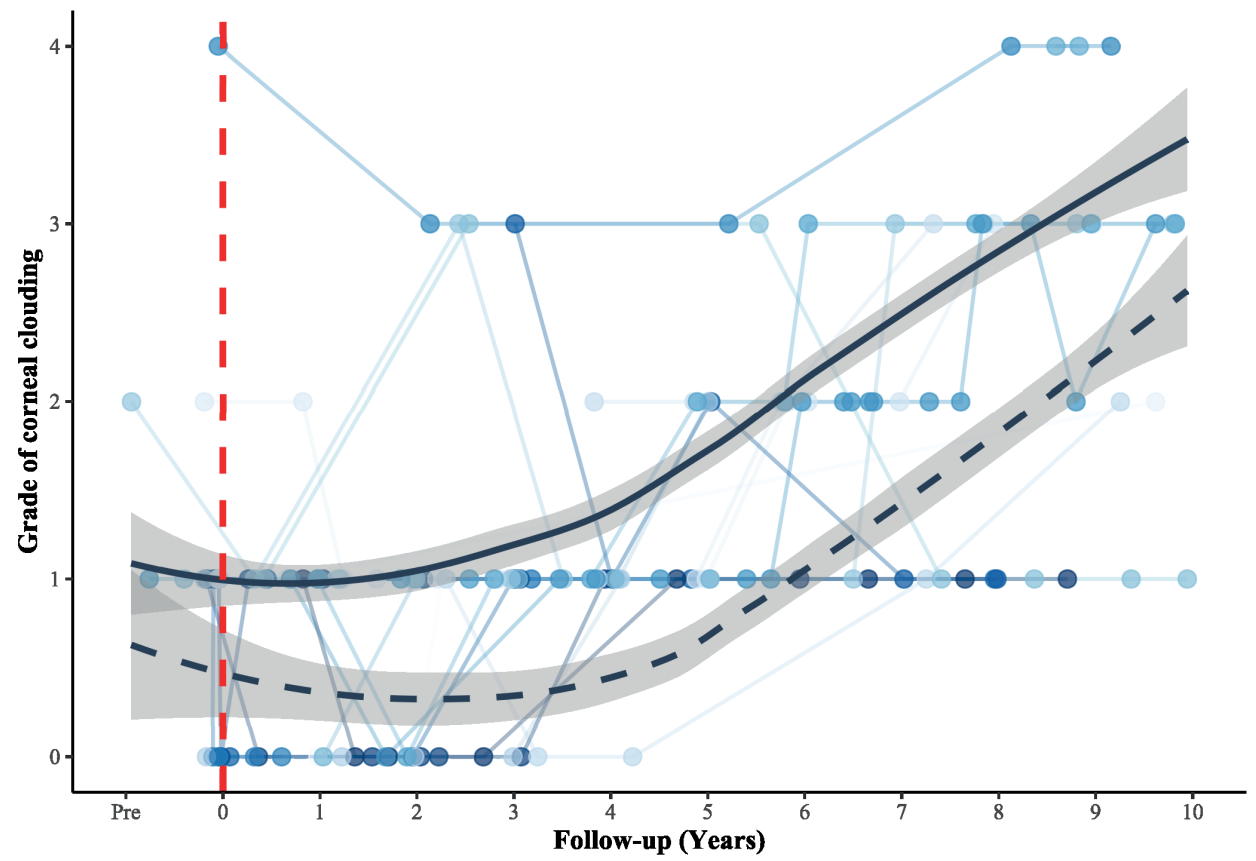

Figure 1. Corneal clouding per individual patient (blue lines; OD) and the course over time as estimated by a linear mixed model for a Hurler (black line) and a Hurler-Scheie (dashed black line) patient with normal IDUA enzyme activity levels after HCT and mean age at transplantation (1.22 years). Dashed vertical red line reflects timing of transplantation. Shaded lines reflect the individual patient data.

\section{Other ocular pathology}

A large number of patients (71\%) showed severe hyperopic refractive errors, ranging from $+4.00 \mathrm{D}$ to $+9.25 \mathrm{D}$ (figure 4), illustrating failure of natural emmetropization, which is the "normal" state of the eye in which accommodation is relaxed and parallel rays of light focus on the retina. ${ }^{13,21}$ Refractive error is influenced by three parameters, namely axial length of the globe, refractive capacity of the lens and refractive capacity of the cornea. Axial length was measured 19 times in 12 patients. Median axial length was $20.96 \mathrm{~mm}$ for patients younger than 4 years and $22.14 \mathrm{~mm}$ for patients above 4 years compared to 20.40 and 22.18 $\mathrm{mm}$, respectively, in healthy children. ${ }^{14}$ None of the children had lens abnormalities. Central corneal thickness (CCT) however, measured 16 times in 11 patients, had a median of $630 \mu \mathrm{m}$ (412 $\mu \mathrm{m}-931 \mu \mathrm{m})$ compared to $565 \mu \mathrm{m}(493 \mu \mathrm{m}-634 \mu \mathrm{m})$ in healthy children. ${ }^{15}$ Increased corneal thickness might lead to increased measured intraocular pressure (IOP). Indeed, our patients showed increasing IOP over time (figure 5). In support, there was a significant positive correlation between CCT and IOP $(\rho=0.56, p=0.03$, data not shown). Four patients received IOP-lowering medication, but none of them required glaucoma surgery during this study's follow-up period. 


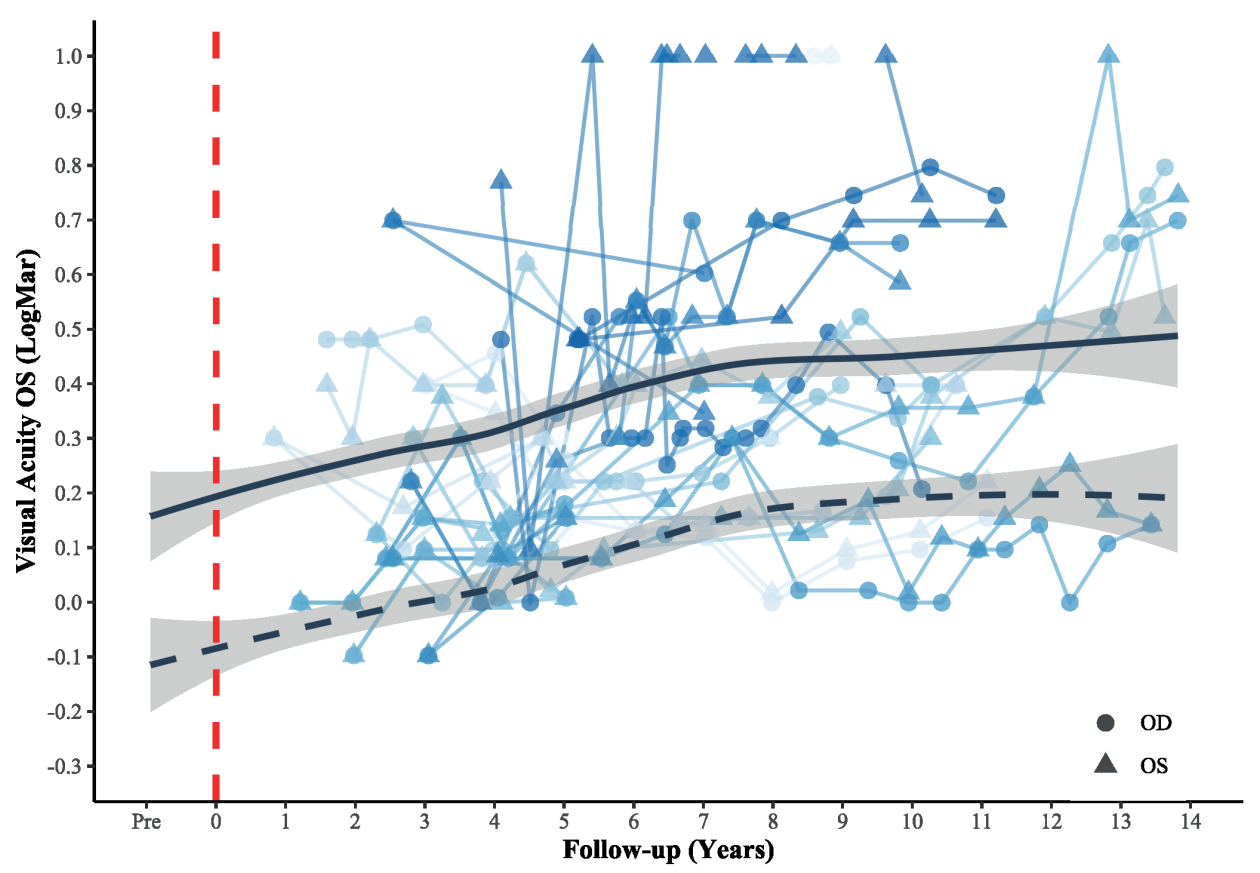

Figure 2. Best Corrected Visual Acuity (LogMar) per individual patient (blue lines; OD and OS) and the course over time as estimated by a linear mixed model for a Hurler (black line) and a Hurler-Scheie (dashed black line) patient with normal IDUA enzyme activity levels after HCT and mean age at transplantation (1.22 years). Dashed vertical red line reflects timing of transplantation. LogMar scale is used to describe vision loss, 0 LogMar represents normal vision, positive LogMar values indicate vision loss and thus poor vision; negative LogMar values indicate good vision. Shaded lines reflect the individual patient data.

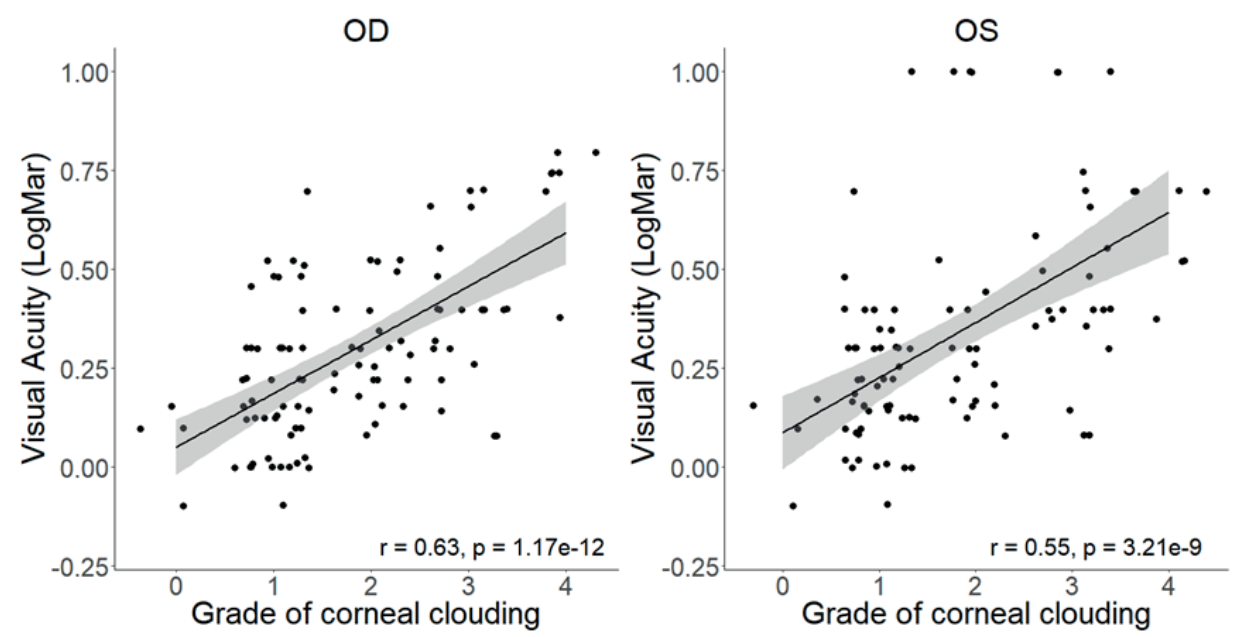

Figure 3. Correlation between Best Corrected Visual Acuity and Corneal clouding. A strong correlation is seen between BCVA and corneal clouding in MPS-1 patients (OD: $\rho=0.63$; p-value $=1.17 \mathrm{e}-12$; OS: $\rho=0.55$; $p$-value $=3.21 \mathrm{e}-9)$. 
Finally, two patients underwent strabismus surgery. One of them at four years of age, the other twice: at seven and ten years of age. In two patients with symptoms of night blindness, retinal degeneration was assessed and confirmed by electroretinography showing a pattern of rod-cone dystrophy. Another patient with severe corneal clouding and low visual acuity showed no abnormalities on VEP at 7 years of age. Other retinal changes were difficult to evaluate as increased corneal clouding impeded analysis of the posterior eye segment. One patient with the Hurler-Scheie phenotype and severe corneal clouding but no signs of retinal degeneration received a corneal transplant at the age of 9 years and continues to have clear corneas after 15 years of follow-up.

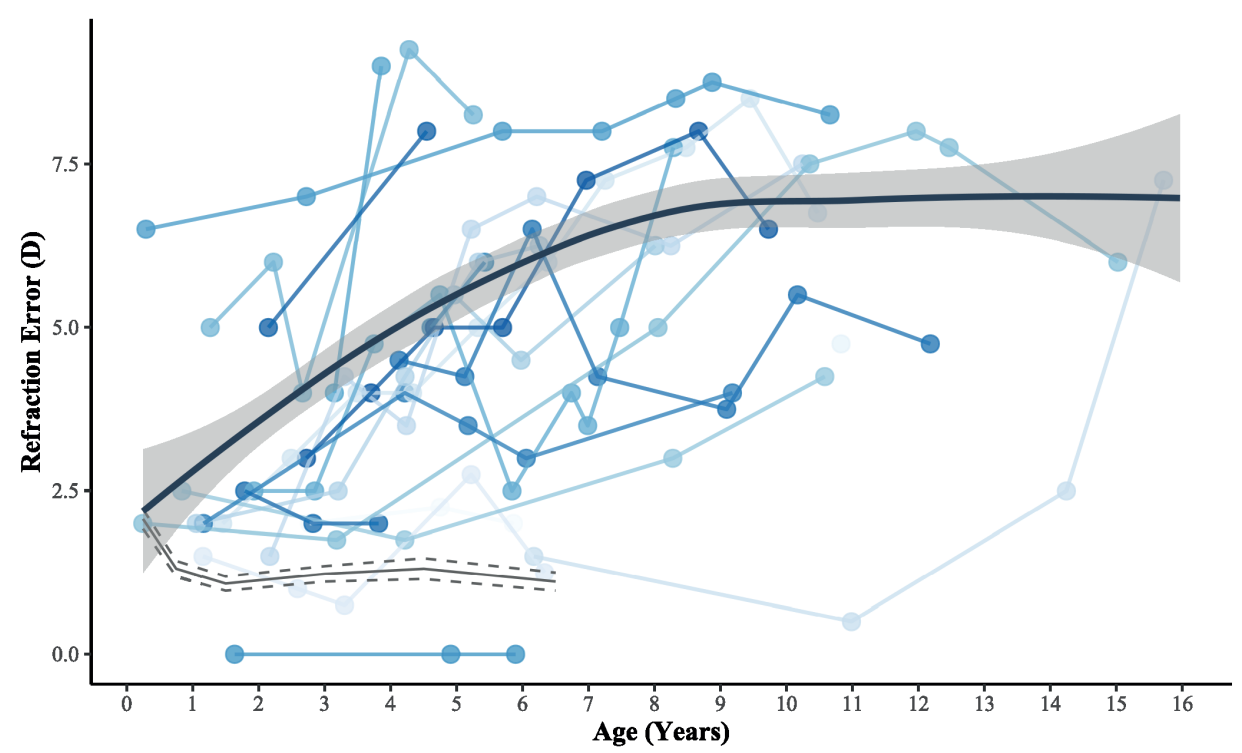

Figure 4. Refractive Error (D) per individual patient (blue lines; OD) and the course over time as estimated by a linear mixed model for a MPS-1 patient (black line) with normal IDUA enzyme activity levels after HCT and mean age at transplantation (1.22 years). Grey lines represent data from healthy children (mean and $95 \% \mathrm{CI}){ }^{13}$

\section{IDUA and AGAL enzyme activity in tear fluid}

To determine the extent to which IDUA, derived from donor blood cells, penetrates into tear fluid as a possible source of enzyme for the cornea, IDUA and AGAL (as a control) activities were measured in MPS-1 patients and healthy controls (figure 6A). IDUA activity in tear fluid of MPS-1 patients after HCT is considerably lower than in that of controls (median 0.05 , range $0.05-0.27 \mathrm{nmol} 4 \mathrm{MU} / \mathrm{h}$. $\mathrm{mg}$ vs. 6.73, range $1.53-11.67 \mathrm{nmol} 4$ MU/h. mg, respectively; $\mathrm{p}<0.0001)$. The AGAL activity also shows a difference but no discrimination between patients (median 0.93, range $0.55-4.34 \mathrm{nmol} 4 \mathrm{MU} / \mathrm{h} . \mathrm{mg}$ ) and controls (median 2.98, range $1.70-6.32 \mathrm{nmol} 4 \mathrm{MU} / \mathrm{h} . \mathrm{mg} ; \mathrm{p}=0.01$ ). After normalization of 
IDUA activity towards the corresponding AGAL activity (IDUA/AGAL activity ratios), the pattern largely resembles the pattern seen for IDUA activity (median 0.07, range $0.01-0.33$ versus median 1.73 , range $0.60-3.03$ in patients versus controls; $p<0.0001$ ). All patients showed hematological IDUA and AGAL enzyme activities in the normal range in dried blood spots after transplantation (1.7 -14.3 nmol $4 \mathrm{MU} / \mathrm{h} . \mathrm{ml}$ and 3.6- $8.8 \mathrm{nmol}$ 4MU/h.ml blood, respectively).

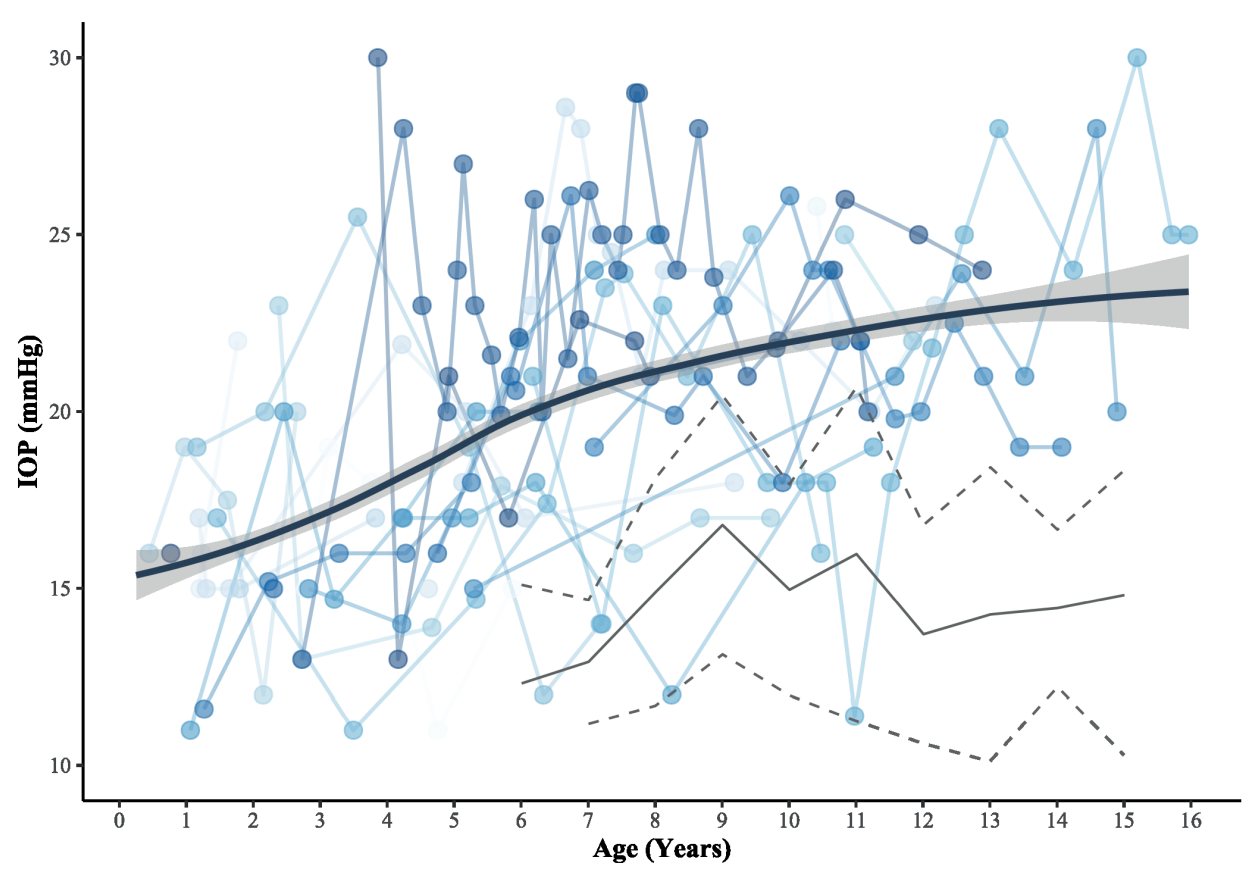

Figure 5. IOP (mmHg) per individual patient (blue lines; OD) and the course over time as estimated by a linear mixed model for a MPS-1 patient (black line) with normal IDUA enzyme activity levels after HCT and mean age at transplantation (1.22 years). Grey lines represent data from healthy children (mean and 95\% CI). ${ }^{16}$

\section{GAG content in tear fluid}

The results of the GAG-concentrations (HS, DS, and KS) in tear fluid are shown in figure 6B. The median concentration of HS was 0.08 (range $0.008-0.251 \mu \mathrm{g} / \mathrm{mg}$ protein) in patients and 0.19 (range $0.06-0.29 \mu \mathrm{g} / \mathrm{mg}$ protein) in controls. The median concentration of KS was 0.002 (range $0.00-0.20 \mu \mathrm{g} / \mathrm{mg}$ protein) in patients and 0.003 (range $0.00-0.59 \mu \mathrm{g} / \mathrm{mg}$ protein) in controls. Finally, the median concentration of DS was 0.002 (range $0.00-0.02 \mu \mathrm{g}$ / $\mathrm{mg}$ protein) in patients and 0.00 (range $0.00-0.003 \mu \mathrm{g} / \mathrm{mg}$ protein) in controls. None of the three GAG species - HS, KS, or DS - in tear fluid of patients differed significantly from the levels found in healthy controls $(\mathrm{p}=0.16, \mathrm{p}=0.87$, and $\mathrm{p}=0.06$, respectively). 
A

IDUA \& AGAL activity

B
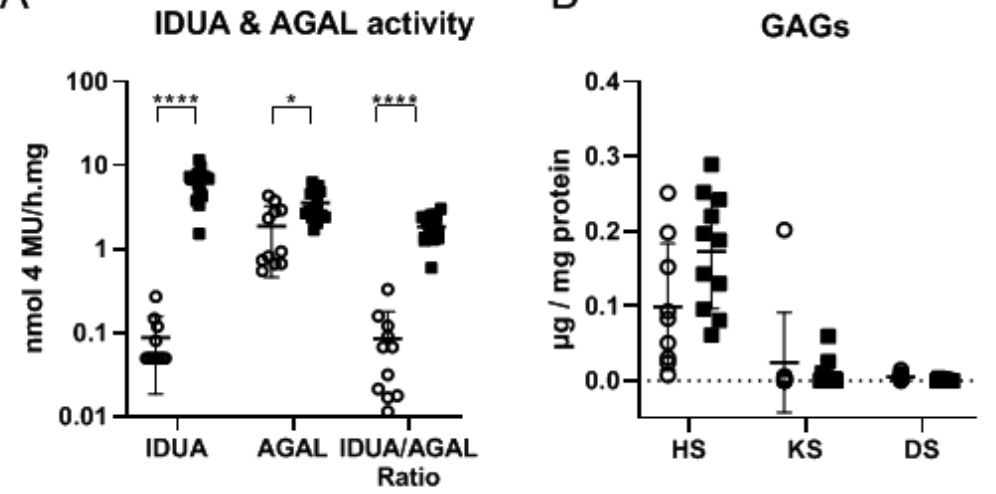

○ MPS-1

- Controls

Figure 6. Lysosomal specific enzyme activities of IDUA and AGAL (A) and concentrations of HS, KS, and DS (B) in MPS-1 patients after transplantation (white dots) and controls (black squares) in tear fluid. $* \mathrm{p}<0.05$, ** $\mathrm{p}<0.01,{ }^{* * *} \mathrm{p}<0.001,{ }^{* * * *} \mathrm{p}<0.0001$.

\section{DISCUSSION}

This multifaceted longitudinal analysis of ocular disease in a unique cohort of children with MPS-1 after HCT reveals ongoing corneal clouding - paralleled by decreasing visual acuity after an initial stabilization. This finding is important both for clinical management, including long-term follow-up, and adequate counseling. Moreover, it underlines the shortcomings of current therapy and the importance of new (additional) therapies to improve late outcomes of MPS-1 patients.

The very long-term and standardized follow-up data, analyzed with a mixed model, allowed us to unite contradictory results of previous studies showing both improvement and worsening of corneal clouding after HCT., ${ }^{1,4,5,12}$ Most studies only evaluated one particular time point or a short time period after HCT, resulting in different conclusions. The observation that clinical phenotype and age at transplantation were important predictors for corneal clouding in MPS1 patients is in concordance with previous research as both have been shown in many studies to be important for clinical outcomes after HCT in these patients. ${ }^{4-6}$ In contrast to what is known in literature, ${ }^{4-6,11}$ in which beneficial effects of normal IDUA enzyme activities in leukocytes on late outcomes are reported, our cohort did not show an effect on the outcomes (clouding, BCVA, refractive error, IOP) of low IDUA enzyme activities after transplantation. Likely, this can be explained by a lack of power for this particular analysis (only two patients had enzyme levels below the normal range). Together, these results show that the current standard treatment (HCT) is in need of improvement to reduce residual disease. 
Previous literature elucidated only small amounts of enzyme are necessary to improve degradation of GAGs in the cornea. ${ }^{22}$ The initial stabilization of corneal clouding suggests that, apparently, the GAGs accumulate over time at such a speed that the amount of IDUA available in the cornea falls short to sufficiently degrade these macromolecules, contributing to worsening of corneal clouding. We hypothesized IDUA enzyme might be reaching the cornea via tear fluid, as this is the main source for nutrients for the avascular cornea. Although hematological IDUA enzyme activity levels normalize after HCT, IDUA activity levels in tear fluid were still extremely low. This is similar to our previous research, ${ }^{17}$ in which we show extremely low IDUA activity in saliva despite treatment with HCT, indicating low enzyme transport from the hematological system to certain tissues. In the eye and other organs with lower perfusion and thus lower delivery of donor-derived IDUA, this might be the explanation for long-term disease progression despite current available treatments. Although there was a considerable overlap, AGAL specific activity in tear fluid from MPS-1 patients was lower than in healthy controls. This was not previously found either in DBS or saliva. ${ }^{17}$ Currently, we do not completely understand this finding. The difference could be a result of the small number of samples included, or that the composition of tear fluid differs among MPS-1 patients and healthy controls. The difference in age between controls (adults) and patients might also be a reason for the observed difference. AGAL measured in DBS, however, does not show a difference between children and adults.

In untreated MPS-1 patients, the excess of GAGs are excreted in the urine. ${ }^{20}$ We examined whether GAGs were also excreted in tear fluid. However, the amounts of the three forms of GAGs were rather low and we did not find a difference in the GAGs concentrations between patients and controls. As emphasized above, ongoing accumulation of GAGs in the cornea is probably the reason for the concurrent ocular pathology we describe (i.e. a large number of patients with hyperopia, increased CCT and IOP). Increased corneal thickness, most likely because of ongoing accumulation of GAGs in the cornea and consecutively resulting in stiffer corneas, probably diminishes the refractive capacity of the cornea and therefore leads to hyperopia in these patients. Furthermore, it is debatable whether the observed increased IOP is truly increased IOP or also the result of stiffness of the cornea which impedes the measurement of the true IOP. ${ }^{23}$

Cornea targeted AAV-gene therapy has shown to be able to transduce keratocytes in the cornea which subsequently produce supra normal activity of IDUA. ${ }^{22}$ Increasing the amount of enzyme in the cornea could be an effective modality to improve clinical ocular outcomes. Whether gene-transduced autologous HCT protocols, using stronger promoters with overexpression of IDUA in the hematological compartment also improves clinical manifestations of the eye awaits evaluation. If not, treatments focusing on increasing the 
60 Chapter 3

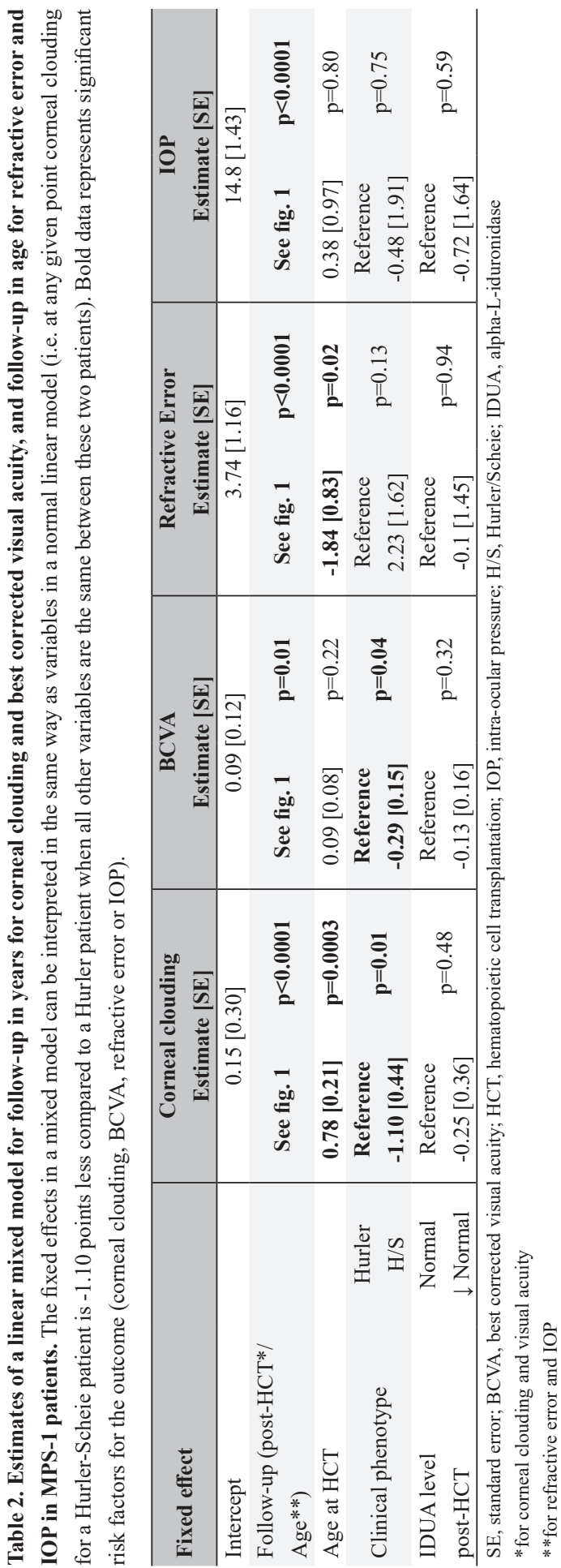


transport of hematological IDUA to the eye or increasing local production of IDUA, such as cornea targeted AAV-gene therapy, should be considered to reduce the clearly present residual disease in the eyes of these patients.

One Hurler-Scheie patient received a corneal transplant with long-term success. In general, corneal transplantation in young children is not advised as the rejection rate in children is high. ${ }^{24}$ Furthermore, retinal degeneration, opticopathy or amblyopia which are also seen in these patients might additionally hamper visual acuity and will not be resolved after a corneal transplant. Therefore, patients should always undergo additional tests like visual field, optical coherence tomography scan, electroretinography, and visual evoked potentials. When these are normal, a corneal transplant could be considered in older patients. ${ }^{25}$

MPS-1 related corneal clouding occurs due to progressive alteration of the morphology and function of the cornea (i.e. disruption of the orderly collagen structure in the stroma of the cornea). ${ }^{26-28}$ Increased amounts of collagen fibrils I, IV and VI are seen, showing both increased spacing and increased mean fibril diameter. ${ }^{27,28}$ Two pathways have been described for this increase. Firstly, GAGs are important for maintaining normal collagen fibril diameter and orientation within the cornea. Therefore, accumulating GAGs are expected to result in disruption of the cornea. ${ }^{29,30}$ Secondly, the transformation of keratocytes into myofibroblasts has been described in corneal injury and is also associated with increased collagen synthesis, leading to corneal haze. ${ }^{31,32}$ Whether this transformation is also happening in MPS-1 patients is being explored in ongoing research. ${ }^{28}$ More insight in the pathophysiology of corneal clouding in MPS-1 patients could help envision additional therapeutic strategies.

Most studies, including ours, that evaluate corneal clouding in MPS-1 patients are limited by the subjective classification. ${ }^{4,33}$ Although patients above the age of 5 could possibly be evaluated with the Pentacam Densitometry Program as described before by Javed et al., an objective method to quantify the level of corneal clouding in all, both young and older, pediatric patients with MPS-1 has not yet been established. ${ }^{34}$ This might introduce greater uncertainty in the results. However, all patients were seen by an ophthalmologist with a high level of experience in MPS-1 patients in order to reduce the inter- and intra-observer variability.

In conclusion, this study describes ongoing ocular disease despite treatment with HCT in patients with MPS-1, resulting in impaired vision representing a burden on these patients. In contrast to the situation in healthy subjects, the IDUA activity in tear fluid in MPS-1 patients is very low, despite HCT. Since it is likely that tear fluid is the main source of IDUA for corneal tissue after transplantation, this would at least partly explain why corneal clouding is a permanent problem. This finding adds to the growing body of evidence of residual disease despite current treatments. Patients undergoing HCT for MPS-1 should be 
informed by their clinicians about the evidence of ongoing disease. Yearly follow-up of these patients by an experienced ophthalmologist, including at least BCVA, refractive error, IOP, grading of corneal clouding, as described by Fahnehjelm et al. ${ }^{3}$ by slit lamp examination, and fundoscopy is necessary to intervene early with occurring ocular manifestations. The question, however, whether novel therapies are capable of addressing residual disease is still begging for an answer.

\section{ACKNOWLEDGEMENTS}

All authors would like to thank all parents and patients for participating in this study. 


\section{REFERENCES}

1. Gullingsrud EO, Krivit W, Summers CG. Ocular abnormalities in the mucopolysaccharidoses after bone marrow transplantation. Longer follow-up. Ophthalmology. 1998; 105: 1099-1105.

2. Ashworth JL, Biswas S, Wraith E, Lloyd IC. Mucopolysaccharidoses and the eye. Surv Ophthalmol. 2006; 51: 1-17.

3. Fahnehjelm KT, Ashworth JL, Pitz S, et al. Clinical guidelines for diagnosing and managing ocular manifestations in children with mucopolysaccharidosis. Acta Ophthalmol. 2012; 90: 595-602.

4. Javed A, Aslam T, Jones SA, et al. The effect of haemopoietic stem cell transplantation on the ocular phenotype in mucopolysaccharidosis type I (Hurler). Acta Ophthalmol. 2017: 1-5.

5. Aldenhoven M, Wynn RF, Orchard PJ, et al. Long-term outcome of Hurler syndrome patients after hematopoietic cell transplantation: an international multicenter study. Blood. 2015; 125: 2164-2172.

6. Boelens JJ, Rocha V, Aldenhoven M, et al. Risk factor analysis of outcomes after unrelated cord blood transplantation in patients with hurler syndrome. Biol Blood Marrow Transplant. 2009; 15: 618-625.

7. Aldenhoven M, Jones SA, Bonney D, et al. Hematopoietic Cell Transplantation for Mucopolysaccharidosis Patients Is Safe and Effective: Results after Implementation of International Guidelines. Biol Blood Marrow Transplant. 2015; 21: 1106-1109.

8. Wang J, Luan Z, Jiang H, et al. Allogeneic Hematopoietic Stem Cell Transplantation in Thirty-Four Pediatric Cases of Mucopolysaccharidosis - A Ten-Year Report from the China Children Transplant Group. Biol Blood Marrow Transplant. 2016; 22: 2104-2108.

9. Eisengart JB, Rudser KD, Xue Y, et al. Long-term outcomes of systemic therapies for Hurler syndrome: an international multicenter comparison. Genet Med. 2018; 20: 1423-1429.

10. Boelens JJ, Aldenhoven M, Purtill D, et al. Outcomes of transplantation using various hematopoietic cell sources in children with Hurler syndrome after myeloablative conditioning. Blood. 2013; 121: 3981-3987.

11. Aldenhoven MA, van den Broek BTA, Wynn RF, et al. Quality of life of Hurler syndrome patients after successful hematopoietic stem cell transplantation. Blood Adv. 2017; 1: 2236-2242.

12. Teär Fahnehjelm K, Olsson M, Chen E, Hengstler J, Naess K, Winiarski J. Children with mucopolysaccharidosis risk progressive visual dysfunction despite 
haematopoietic stem cell transplants. Acta Paediatr. 2018:1-9.

13. Mayer DL, Hansen RM, Moore BD, Kim S, Fulton AB. Cycloplegic refractions in healthy children aged 1 through 48 months. Arch Ophthalmol. 2001; 119: 1625-1628.

14. Mutti DO, Lynn Mitchell G, Sinnott LT, et al. Ocular Component Development during Infancy and Early Childhood. Optom Vis Sci. 2018; 95: 976-985.

15. Pediatric Eye Disease Investigator Group. Central Corneal Thickness in Children. Arch Ophthalmol. 2011; 129: 1132-1138.

16. Dusek WA, Pierscionek BK, McClelland JF. Age variations in intraocular pressure in a cohort of healthy Austrian school children. Eye. 2012; 26: 841-845.

17. Doorn J van, van den Broek BTA, Geboers AJ, Kuiper G-A, Boelens JJ, van Hasselt PM. Salivary $\alpha$-Iduronidase Activity as a Potential New Biomarker for the Diagnosis and Monitoring the Effect of Therapy in Mucopolysaccharidosis Type I. Biol Blood Marrow Transplant. 2018; 24: 1808-1813.

18. Rentka A, Koroskenyi K, Harsfalvi J, et al. Evaluation of commonly used tear sampling methods and their relevance in subsequent biochemical analysis. Ann Clin Biochem. 2017.

19. Chamoles NA, Blanco MB, Gaggioli D, Casentini C. Hurler-like Phenotype. Clin Chem. 2001;47.

20. Langereis EJ, Wagemans T, Kulik W, et al. A Multiplex Assay for the Diagnosis of Mucopolysaccharidoses and Mucolipidoses. Karamanos NK, ed. PLoS One. 2015; 10.

21. Brown NP, Koretz JF, Bron AJ. The development and maintenance of emmetropia. Eye. 1999; 13: 83-92.

22. Vance M, Llanga T, Bennett W, et al. AAV Gene Therapy for MPS1-associated Corneal Blindness. Sci Rep. 2016; 6: 2-11.

23. Fahnehjelm KT, Chen E, Winiarski J. Corneal hysteresis in mucopolysaccharidosis I and VI. Acta Ophthalmol. 2012; 90: 445-448.

24. Limaiem R, Chebil A, Baba A, Ben Youssef N, Mghaieth F, El Matri L. Pediatric Penetrating Keratoplasty: Indications and Outcomes. Transplant Proc. 2011; 43: 649-651.

25. Ohden KL, Pitz S, Ashworth J, et al. Outcomes of keratoplasty in the mucopolysaccharidoses: an international perspective. Br J Ophthalmol. 2017; 101: 909-912.

26. Constantopoulos G, Scott JA, Shull RM. Corneal opacity in canine MPS I. Changes after bone marrow transplantation. Investig Ophthalmol Vis Sci. 1989; 30: 1802-1807.

27. Huang Y, Bron AJ, Meek KM, Vellodi A, McDonald B. Ultrastructural study of the 
cornea in a bone marrow-transplanted Hurler syndrome patient. Exp Eye Res. 1996; 62: 377-387.

28. Yuan C, Bothun ED, Hardten DR, Tolar J, McLoon LK. A novel explanation of corneal clouding in a bone marrow transplant-treated patient with Hurler syndrome. Exp Eye Res. 2016; 148: 83-89.

29. Borcherding MS, Blacik LJ, Sittig RA, Bizzell JW, Breen M, Weinstein HG. Proteoglycans and collagen fibre organization in human corneoscleral tissue. Exp Eye Res. 1975; 21: 59-70.

30. Rada JA, Cornuet PK, Hassell JR. Regulation of corneal collagen fibrillogenesis in vitro by corneal proteoglycan (lumican and decorin) core proteins. Exp Eye Res. 1993; 56: 635-648.

31. Jester J V., Petroll WM, Cavanagh HD. Corneal stromal wound healing in refractive surgery: the role of myofibroblasts. Prog Retin Eye Res. 1999; 18: 311-356.

32. Moller-Pedersen T, Li H, Petroll WM. Confocal microscopic characterization of wound repair after photorefractive keratectomy using in vivo confocal microscopy. Investig Ophthalmol Vis Sci. 1998; 39: 487-501.

33. Summers CG, Fahnehjelm KT, Pitz S, et al. Systemic therapies for mucopolysaccharidosis: Ocular changes following haematopoietic stem cell transplantation or enzyme replacement therapy - a review. Clin Exp Ophthalmol. 2010; 38: 34-42.

34. Javed A, Aslam T, Jones SA, Ashworth J. Objective quantification of changes in corneal clouding over time in patients with mucopolysaccharidosis. Investig Ophthalmol Vis Sci. 2017; 58: 954-958. 


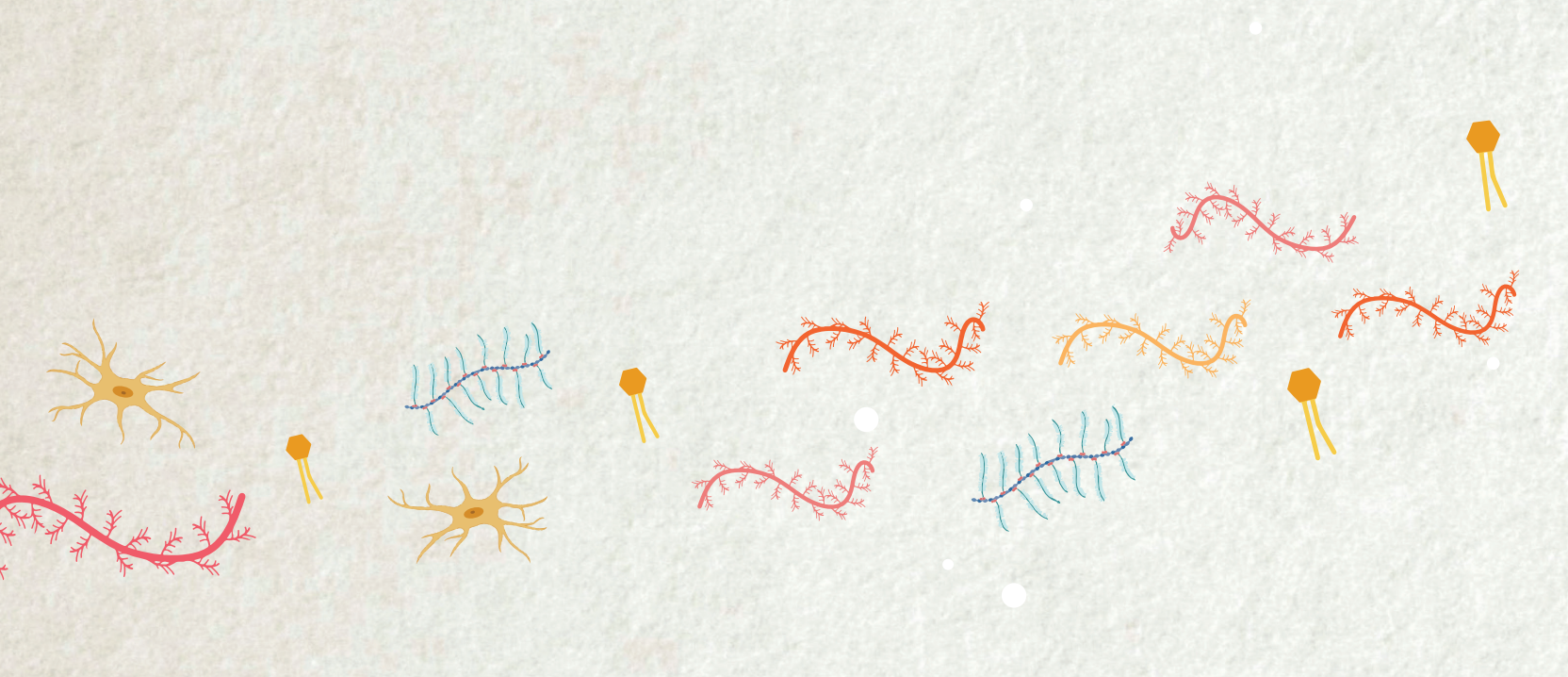



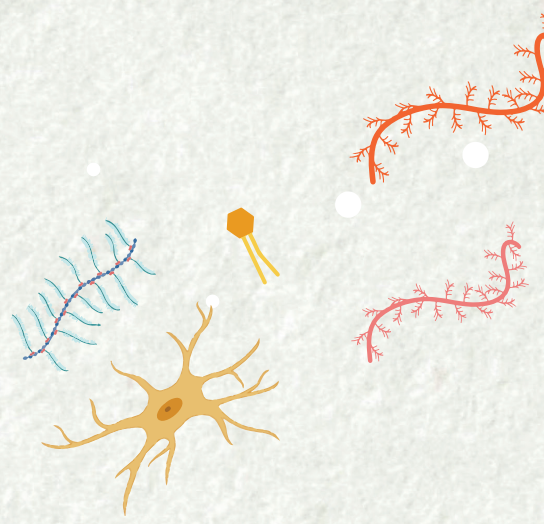

\section{CHAPTER 4}

Hearing Loss in Patients with

Mucopolysaccharidoses after Hematopoietic

Cell Transplantation; a Longitudinal Analysis

Brigitte T.A. van den Broek

Adriana L. Smit

Jaap Jan Boelens

Peter M. van Hasselt 


\section{ABSTRACT}

Background: Hearing loss is frequently seen in Mucopolysaccharidoses (MPS) patients. Although hematopoietic cell transplantation (HCT) increases overall survival, disease progression is observed in certain tissues. This study describes the course of hearing loss (HL) over time in transplanted MPS patients.

Methods: Transplanted MPS patients between 2003 and 2018 were included and received yearly audiological evaluation, including auditory brainstem response (ABR) or pure tone audiometry (PTA). Additionally, MRIs of the cerebellopontine angle were performed.

Results: 28 MPS-1, 2 MPS-2, 2 MPS-3, 2 MPS-4, and 4 MPS-6 patients were analyzed with a median follow-up of 5 years (range 0.9-15.9 years). Air conduction threshold improved significantly over time $(\mathrm{p}<0.001)$ with a PTA 1 year post-HCT of $50 \pm 0.7 \mathrm{~dB}$ to $23 \pm 11 \mathrm{~dB}$ 13 years post-HCT. Bone conduction threshold worsened with a PTA 1 year post-HCT of $10 \pm 7 \mathrm{~dB}$ to $18 \pm 9 \mathrm{~dB} 13$ years post-HCT $(\mathrm{p}=0.34)$. The degree of HL varied from mild-severe early after HCT to normal-mild at longer follow-up. The type of HL shifted from conductive in the first years post-HCT to sensorineural at longer follow-up. MRIs of the cerebellopontine angle did not show abnormalities.

Conclusions: HL is still seen in patients with MPS despite HCT and shifts from a conductive type early after HCT to a sensorineural type at longer follow-up. Yearly follow-up of HL is necessary to timely intervene, as hearing is important in the speech and language development of children and their academic achievements. 


\section{INTRODUCTION}

Hearing loss is a common feature in all subtypes of the mucopolysaccharidoses (MPS). ${ }^{1-7}$ The MPS are lysosomal storage disorders caused by a deficiency of one of the enzymes involving glycosaminoglycan (GAG) degradation, leading to multi-systemic disease and, if severe, premature death. ${ }^{8}$ Undiagnosed MPS patients are frequently evaluated by otorhinolaryngologists because of the early-onset of ear-nose-throat (ENT) manifestations, including otitis media, macroglossia, adenotonsillar hypertrophy, nasal obstruction, obstructive sleep apnea syndrome (OSAS), progressive respiratory disorders, and hearing loss. ${ }^{9}$

Hearing loss in MPS may be conductive, sensorineural or mixed in origin. The conductive component of hearing loss is the result of seromucinous otitis, ossicular chain deformities or

disruption, or arthropathy. ${ }^{10,11}$ Sensorineural hearing loss (SNHL) has been attributed to the accumulation of GAGs in the cochlea, auditory nerve, and brain stem. ${ }^{12}$

Hematopoietic cell transplantation is used as treatment for MPS-1, MPS-2, MPS-3, MPS-4 and MPS- $6 .{ }^{13}$ Based on the principle of cross-correction, HCT has dramatically increased life expectancy in these patients in the past decade. ${ }^{14}$ In depth analyses, however, have shown disease progression in some organs but not in others despite optimized transplantation protocols. ${ }^{15,16}$ Detailed long-term outcome studies on hearing loss in MPS patients after HCT are scarce and most often cross-sectional. ${ }^{17,18}$ The extensive follow-up program at the 'Sylvia Toth Center for Multidisciplinary Follow up after Hematopoietic Cell Transplantation' in the Wilhelmina Children's Hospital, Utrecht, The Netherlands, has over 15 years of experience with these patients. This allows us to perform in depth longitudinal analyses and analyze the course of hearing loss in transplanted MPS patients, which are reported in this study.

\section{PATIENTS AND METHODS}

\section{Data collection}

All MPS patients treated with HCT in the Wilhelmina Children's Hospital (WKZ) of the University Medical Centre Utrecht (UMCU), The Netherlands between 2003 and 2019, were included in this study and evaluated by an experienced otorhinolaryngologist and received a hearing assessment on a yearly basis at the 'Sylvia Toth Center for Multidisciplinary Follow up after Hematopoietic Cell Transplantation'. This study is based on retrospectively collected patient data acquired during care-as-usual and has been exempted for approval according to the responsible institutional committee Medical Research Involving Human Subjects Act. The MPS phenotype, age at transplantation, enzyme activity level after transplantation (scored as 
below or above lower limit of normal, cut-off point: $20 \mathrm{nmol} / \mathrm{h} / \mathrm{mg}$ ), otorhinolaryngological and audiological interventions, including the insertion of a ventilation tube in the ear drum and hearing aid use were collected from the medical records.

\section{Audiologic examination}

Pure tone audiometry was performed repeatedly to assess hearing levels. In uncooperative patients due to a young age or cognitive impairment, auditory brainstem response (ABR) was performed during natural sleep or sedation. The hearing loss was evaluated according to the threshold in the better ear. The results of pure tone audiometry and ABR were analyzed separately.

An experienced audiology assistant performed the hearing test by air (AC) and bone conduction (BC) for the frequencies 250,500, 1000, 2000, 4000, and $8000 \mathrm{~Hz}$ in a quiet room and masking as appropriate. The hearing loss levels were determined by taking the four-frequency pure tone average (PTA) of the air conduction threshold (500-1000-2000$4000 \mathrm{~Hz})$.

The degree of hearing loss was categorized according to the World Health Organization (WHO) International Classification of Impairments, Disabilities, and Handicaps (WHOICIDH) standard. ${ }^{19}$ Hearing loss was classified as normal (0-25 dB), mild (26-40 dB), moderate (41-60 dB), severe (61-80 dB), or profound ( $\geq 81 \mathrm{~dB})$.

The criteria of the Genetic Deafness study group ${ }^{20}$ were used for the classification of the type of hearing loss. The air-bone gap was considered normal if the average AC threshold was $\leq 15 \mathrm{~dB}$ above the $\mathrm{BC}$ threshold. Pure conductive hearing loss ( $\mathrm{CHL}$ ) was defined as an average $\mathrm{BC}$ threshold $\leq 20 \mathrm{~dB}$ with an average air-bone gap $\geq 15 \mathrm{~dB}$. Pure sensorineural hearing loss (SNHL) was defined as an average $\mathrm{BC}$ threshold $>20 \mathrm{~dB}$ with an average airbone gap $<15 \mathrm{~dB}$. And finally, mixed hearing loss (MHL) was defined as an average $\mathrm{BC}$ threshold $>20 \mathrm{~dB}$ with an average air-bone gap of $\geq 15 \mathrm{~dB}$.

\section{Magnetic Resonance Imaging (MRI)}

For the MRI's of the cerebellopontine angle, sagittal spin-echo T1-weighted images, and transverse turbo spin-echo T2-weighted and diffusion weighted single-shot images were performed. Furthermore, transverse 3D T1 and T2-weighted images were performed. The transversal 3D weighted-image was repeated after administration of gadolinium. All images were performed on a Philips Achieva, Ingenia, or Ingenia Elition 1.5 Tesla or 3.0 Tesla magnetic resonance system. 


\section{Statistical analysis}

Baseline characteristics are reported as frequencies and percentages for categorical variables and median (range) for continuous variables. The course of hearing loss over time after transplantation was analyzed with a linear mixed model. To account for non-linearity in air conduction and the air-bone gap, restricted cubic splines with three knots were added to the model. The four-frequency PTA of the better ear was used as the dependent variable for AC and $\mathrm{BC}$ thresholds as well as for the air-bone gap. Based on previous literature, age at time of transplantation, phenotype, and enzyme level after transplantation were considered important covariates and included as fixed effects. A random intercept was included per individual to account for individual variation at baseline and a random slope (for time) for dependency across the repeated measurements within the same individual during follow-up. To analyze the course of hearing loss for the separate frequencies, data of both ears was included. To account for dependency between both ears a random intercept per ear was included additional to the random intercept per individual. Final coefficients were estimated using restricted maximum likelihood and significance of parameters was assessed with a likelihood ratio test between the model with and without the parameter. The model assumptions including normal distributed residuals, random effects, and homogeneity of variance were confirmed visually. P-values $<0.05$ were considered significant. The R project (RStudio: Integrated Development for R. RStudio, Inc., Boston, MA) for statistical computing version 3.4.1 with the packages 'Ime4', 'rms', and 'ggplot2' was used for all analyses and for the creation of figures.

\section{RESULTS}

\section{Patients}

In total, 196 hearing observations of 38 patients were analyzed. MPS-1 was the most frequent MPS subtype ( $\mathrm{n}=28 ; 74 \%$ ). Within the phenotypic range of MPS-1, Hurler was more frequent $(n=26 ; 93 \%)$ compared to Hurler-Scheie $(n=2 ; 7 \%)$. Two patients had MPS-2, two had MPS3 , and two had MPS-4 (5\% each). Finally, four patients had MPS-6 (11\%). The median age at transplantation for all patients was 1.2 years (range $0.2-4.8$ years). Nearly all patients had leukocyte enzyme activity levels in the normal range post-HCT $(n=36,95 \%)$. The median follow-up after HCT consisted of 5.0 years (range $0-16$ years).

\section{Hearing loss}

$A B R$

ABR was performed in 14 patients aged between 0 and 6 years. In 6 patients (43\%) ABR was performed before HCT and all these patients were $<2$ years. Post-HCT ABR were performed in patients aged $2-6$ years with a median follow-up of 1.5 years (range $1-3$ years). The 
Table 1. Patient characteristics

\begin{tabular}{|c|c|c|}
\hline Patient characteristics $(\mathrm{N}=25)$ & $\mathbf{N}(\%)$ & \\
\hline MPS-1 & $28(74 \%)$ & \\
\hline Hurler & $26(93 \%)$ & \\
\hline Hurler-Scheie & $2(7 \%)$ & \\
\hline MPS-2 & $2(5 \%)$ & \\
\hline MPS-3 & $2(5 \%)$ & \\
\hline MPS-4 & $2(5 \%)$ & \\
\hline MPS-6 & $4(11 \%)$ & \\
\hline Age in years at transplantation (median; range) & $1.2(0.2-4.8)$ & \\
\hline Enzyme level above LLN post-HCT & $36(95 \%)$ & \\
\hline Follow-up in years after HCT (median; range) & $5.0(0.9-15.9)$ & \\
\hline dB Hearing loss at follow-up & Air $($ mean \pm SD) & Bone $($ mean $\pm \mathrm{SD})$ \\
\hline 1 year post-HCT $(n=3)$ & $50 \pm 0.7$ & $10 \pm 7$ \\
\hline 3 years post-HCT $(n=14)$ & $34 \pm 16$ & $12 \pm 11$ \\
\hline 5 years post-HCT $(n=15)$ & $30 \pm 15$ & $15 \pm 13$ \\
\hline 10 years post-HCT $(n=7)$ & $24 \pm 6$ & $14 \pm 8$ \\
\hline 13 years post-HCT $(n=4)$ & $23 \pm 11$ & $18 \pm 9$ \\
\hline
\end{tabular}

MPS, Mucopolusaccharidosis; LLN, lower level of normal; HCT, hematopoietic cell transplantation; dB, decibel; SD, standard deviation

average hearing threshold determined by ABR pre-HCT was $43 \mathrm{dBeHL}$ (dB equivalent hearing level). The average hearing threshold determined by ABR post-HCT was $44 \mathrm{dBeHL}$. None of these patients had normal hearing with a threshold of $<20 \mathrm{~dB}$ hearing loss in both ears. A conductive or mixed type hearing loss was seen in the majority of the patients $(n=12$; $86 \%$ ). The two patients with elevated hearing levels measured by ABR determined as SNHL were both MPS-1 patients.

$\mathbf{A}$

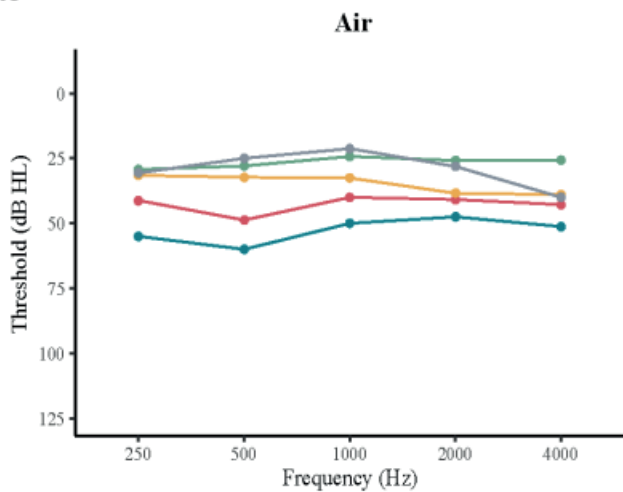

B

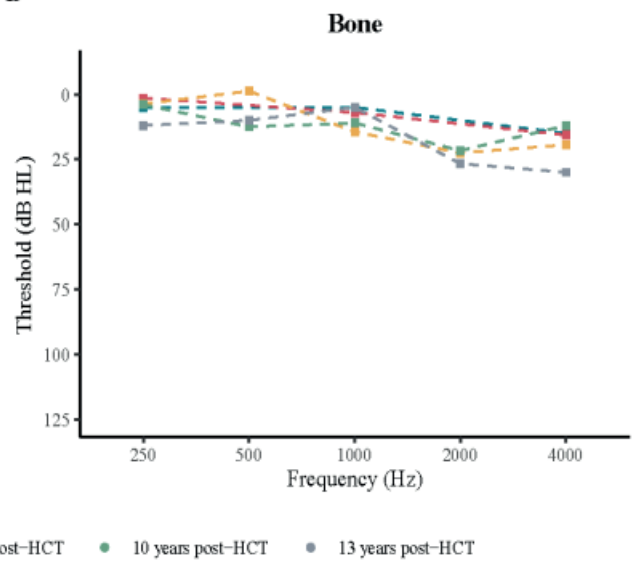

Figure 1. Average pure tone audiogram for air conduction (A) and bone conduction (B) at 1 year (blue), 3 years (red), 5 years (yellow), 10 years (green), and 13 years (grey) after HCT. 


\section{Pure tone audiometry}

Pure tone audiometry was performed longitudinally 182 times in 25 patients from the age of 3 years and onwards (maximum age at latest follow-up was 16.9 years). One year after HCT, the air conducted mean PTA was $50 \mathrm{~dB}(95 \%$ CI 49-51 dB), after 3 years this was 34 $\mathrm{dB}(95 \%$ CI 26-44 dB), after 5 years $30 \mathrm{~dB}$ (95\% CI 22-37 dB), after 10 years $24 \mathrm{~dB}(95 \%$ CI 20-28 dB), and after 13 years $23 \mathrm{~dB}$ (95\% CI 12-33 dB) (table 1; figure 1). Over time, a significant decrease in air conduction threshold was seen $(\mathrm{p}<0.001$; figure $2 \mathrm{~A})$, which correlates with improved hearing. The improvement in hearing was most profound in the first 5 years following HCT. Age at time of transplantation, enzyme activity level after HCT, and phenotype did not show to be significant predictors for the outcome in this cohort (table 2).

$\mathbf{A}$

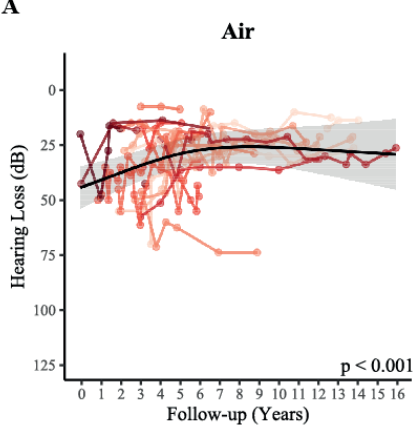

B

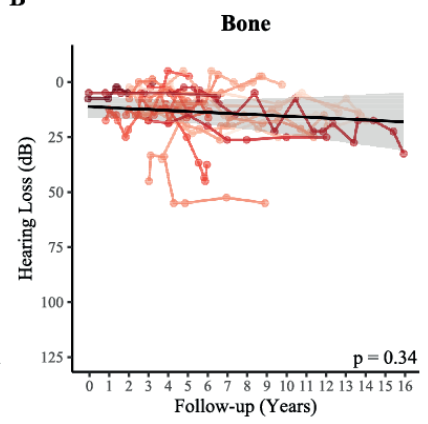

C

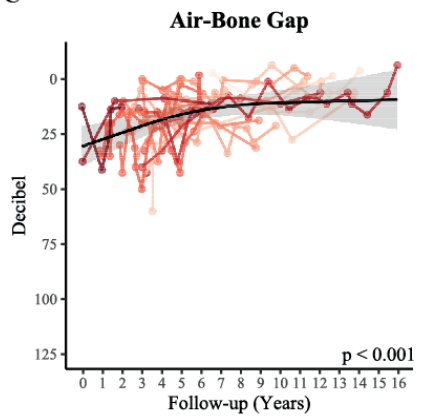

Figure 2. Pure tone average thresholds for air conduction (A), bone conduction (B), and the Air-Bone Gap (C). Air conduction, bone conduction and air-bone gap PTA per individual patient over time (red lines). The black line reflects the average course over time.

Table 2. Linear Mixed-Model Estimates for air conduction threshold, bone conduction threshold, and airbone gap.

\begin{tabular}{ll|cc|cc|cc}
\hline \multirow{2}{*}{ Fixed Effect } & \multicolumn{2}{|c|}{ AC threshold } & \multicolumn{2}{c|}{ BC threshold } & \multicolumn{2}{c}{ Air-Bone Gap } \\
& & Estimate [SE] & $\mathbf{P}$ & Estimate [SE] & $\mathbf{P}$ & Estimate [SE] & P \\
\hline Intercept & & $45.5[7.29]$ & & $7.48[4.7]$ & & $30.1[5.7]$ & \\
Follow-up (years) & & See fig. 2 & $<\mathbf{0 . 0 0 1}$ & See fig. 2 & 0.34 & See fig. 2 & $<\mathbf{0 . 0 0 1}$ \\
\multirow{2}{*}{ Age at HCT } & & $-0.0009[0.01]$ & 0.96 & 0.006 & 0.33 & $0.001[0.007]$ & 0.76 \\
& & & & {$[0.007]$} & & & \\
& MPS1 & Reference & & Reference & & Reference & \\
Clinical phenotype & MPS2 & $-9.6[15.6]$ & \multirow{2}{*}{0.45} & NA & \multirow{2}{*}{0.20} & NA & 0.74 \\
& MPS3 & $-19.6[15.3]$ & & $-8.0[10.1]$ & & $-9.01[12.7]$ & \\
Enzyme activity & MPS6 & $-7.0[11.5]$ & & $-12.8[8.1]$ & & $0.53[8.2]$ & \\
post-HCT & Normal & Reference & \multirow{2}{*}{0.93} & Reference & \multirow{2}{*}{0.53} & Reference & \multirow{2}{*}{0.31} \\
\hline
\end{tabular}

$\mathrm{AC}$, air conduction; $\mathrm{BC}$, bone conduction, SE, standard error; HCT, hematopoietic cell transplantation; NA, not applicable 

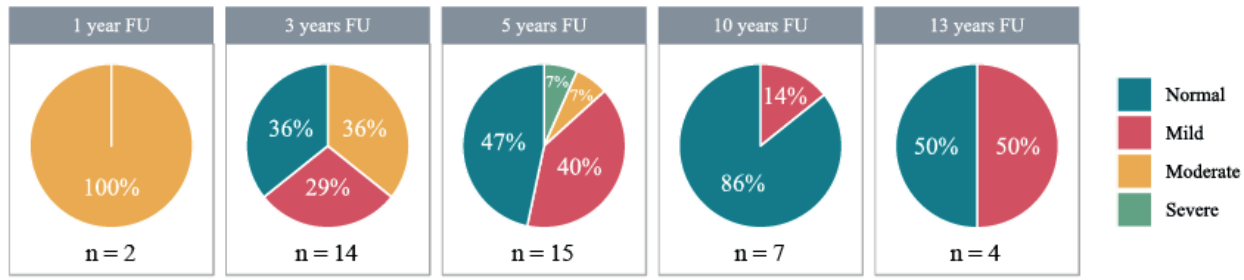

Figure 3. Degree of hearing loss according to WHO criteria at different follow up time points.

The bone conducted mean PTA showed a tendency to increase over time but was not significant $(\mathrm{p}=0.34$; figure $2 \mathrm{~B})$. When looking at the separate frequencies, the increase in bone conduction threshold was most profound in the middle range frequencies and significant in the $500 \mathrm{~Hz}$ frequency ( $\mathrm{p}=0.007$; suppl. figure 1). One year after HCT, the bone conducted mean PTA was $10 \mathrm{~dB}(95 \%$ CI 3-18 dB), after three years it was $12 \mathrm{~dB}(95 \% \mathrm{CI}$ 6-17 dB), after 5 years $15 \mathrm{~dB}(95 \%$ CI 8-21 dB), after 10 years $14 \mathrm{~dB}(95 \% \mathrm{CI} 8-20 \mathrm{~dB})$, and after 13 years $18 \mathrm{~dB}(95 \%$ CI 9-26 dB) (table 1; figure 1). In this cohort, bone conduction was not significantly affected by the age at transplantation, the enzyme activity level after transplantation, or phenotype (table 2 ).

According to the WHO criteria defining hearing loss, $100 \%$ of patients had moderate hearing loss 1 year after HCT $(n=2)$. Three years after HCT, 36\% had normal, 29\% mild, and 36\% moderate hearing loss ( $\mathrm{n}=14$; figure 3$)$. At 5 years after HCT, 47\% had normal hearing, $40 \%$ mild hearing loss, $7 \%$ moderate, and $7 \%$ severe $(n=15)$. Ten years after HCT, $86 \%$ had normal hearing versus $14 \%$ with mild hearing loss $(n=7)$. Finally, at 13 years after HCT $50 \%$ had normal hearing and $50 \%$ mild hearing loss $(n=4$; figure 3$)$. Of the patients with $>10$ years of follow-up, $89 \%$ improved in the first 5 years of follow-up. Between 5 years of follow-up and their latest follow-up time point, $30 \%$ worsened.

The type of hearing loss shifted from $100 \%$ conductive 1 year after HCT $(n=2)$ to $100 \%$ sensorineural 13 years after HCT ( $\mathrm{n}=4$; figure 4). Three years after HCT $90 \%$ was conductive and $10 \%$ sensorineural $(\mathrm{n}=14), 5$ years after HCT $62 \%$ was conductive, $25 \%$ sensorineural and $12 \%$ mixed $(n=15)$, and 10 years after HCT $33 \%$ was conductive and $67 \%$ sensorineural $(\mathrm{n}=7$; figure 4).
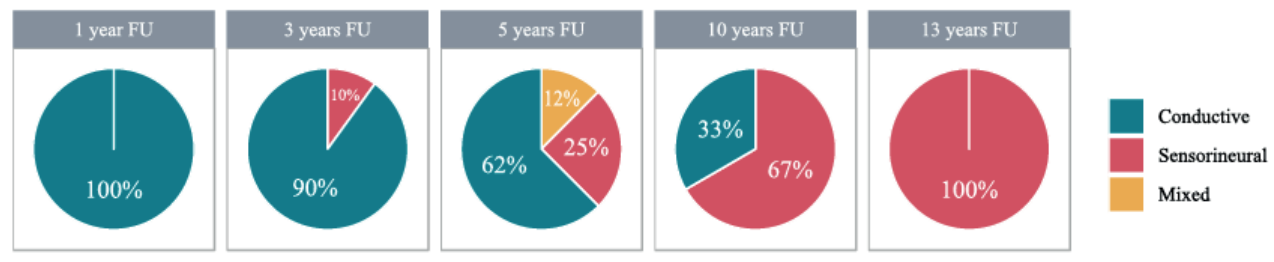

Figure 4. Type of hearing loss according to Genetic Deafness study group at different follow up time points 


\section{Hearing aids or interventions}

Bilateral grommets were placed in 26 of 38 patients (69\%) and 19 patients $(73 \%)$ received these $\geq 2$ times. Of the 38 MPS patients, 15 patients were prescribed hearing amplifications (39\%). The majority used bilateral hearing aids (87\%), with the exception of 2 patients who received a bone conduction device $(13 \%)$. The median age at the start of hearing aids use was 4.4 years (range $1.9-14.9$ years) at a median follow-up of 2.6 years (range $0.8-13.7$ years).

MRI

MRIs of the cerebellopontine angle were performed in 10 patients at median follow-up of 11.9 years (range $4.0-16.4$ years). None showed any abnormalities in the n. cochlearis, $\mathrm{n}$. facialis, or n. vestibularis inferior/superior, respectively. Furthermore, no abnormalities in the anatomy of the cochlea or inner ear were seen.

\section{DISCUSSION}

This longitudinal analysis, spanning more than 15 years of repeated audiological evaluation in MPS children after HCT at a young age, shows hearing impairment despite HCT. Although an initial beneficial effect is seen, a significant number of MPS patients will likely need hearing aids before reaching adulthood. The initial improvement is seen in air conduction in the first 5 years of life after HCT. Thereafter, air conduction thresholds are more or less stable with a mean hearing loss of $20-30 \mathrm{~dB}$. Bone conduction, however, shows a slow deterioration over time which could eventually lead to the need of a hearing aid or even a cochlear implant. This knowledge is important for caregivers counselling these patients.

Detailed analysis of hearing after HCT in MPS patients was reported previously in two other studies and the results are in line with our data. ${ }^{17,18}$ Both were, however, cross-sectional studies and could not show the transition of conductive to sensorineural hearing loss, although they did speculate this hypothesis. Previous studies have shown that treatment at an early age and enzyme activity levels in the normal range are important predictors for the outcome of MPS-1 patients, including hearing..$^{15,21,22}$ For the use of hearing aids, Aldenhoven et al. ${ }^{15}$ showed in a large study with 217 MPS-1 patients a hazard ratio of 0.42 for patients with enzyme activity levels in the normal range compared to patients with enzyme activity level below the normal range. In our cohort however, age at transplantation and enzyme activity level post-transplantation did not show to be predictors for hearing loss, which could be because of the small sample size or the small variation of these two variables (the mean age at transplantation was low at 1.22 years and the cohort was homogeneous with only two patients having enzyme activity levels below the normal range). 
Eustachian tube dysfunction, recurrent middle ear infections and thickened middle ear mucosa by GAG deposits have been considered important factors of conductive hearing loss in MPS patients. The improvement of air conduction in the first years after HCT might be due to a combination of the natural improvement in the occurrence of chronic otitis in young children by age and a beneficial effect of HCT especially on the mucosal tissue, both resulting in a reduction of otitis episodes. ${ }^{17}$ Furthermore, some patients received ventilation tubes because of otitis media with effusion which will have had a positive effect on the air conduction threshold as well by reduction of the fluid related attenuation. It is important to realize that the mean PTA at 13 years follow-up, when most children have reached early adulthood, was at the borderline between normal hearing and mild hearing loss. Due to natural deterioration of inner ear function by aging, this means the majority of patients will develop significant hearing loss in adulthood. While the air conduction threshold decreases over time, the airbone gap approaches 0 , implying the remaining hearing loss is mostly due to SNHL (defined as bone conduction threshold $>20 \mathrm{~dB}$ with an AB-gap $<15 \mathrm{~dB}$ ).

In contrast to the air conducted threshold, the bone conducted threshold slowly increases over time especially in the middle range frequencies. This implies that hearing loss actually is progressing despite HCT. As disease progression is seen in transplanted patients especially in connective tissue (i.e. skeleton and cornea) $)^{15,16}$, it is reasonable to assume this is also the cause for progressive hearing loss post-HCT. However, we cannot exclude that the hearing loss is the result of toxic effects of HCT itself or a late effect of the otitis episodes early in life. How the bone conduction threshold will develop after even longer follow-up is uncertain, but it is likely that it continues to worsen slowly in addition to the natural deterioration of inner ear function by aging. When severe to profound SNHL is present a cochlear implant could be considered, which has already shown to improve hearing in patients with MPS-1, 2 and 4..$^{23,24}$

The exact mechanism of SNHL in MPS patients is unknown, however Kariya et al. ${ }^{25}$ described the inner ear changes of 6 post-mortem MPS-1 patients. The organs of Corti were affected and the mean number of both inner and outer hair cells was decreased in MPS-1 patients compared to healthy controls. Animal studies on the histopathological changes of the inner ear show similar results. ${ }^{26,27}$ The successful reports of cochlear implant performance in MPS patients support the hypothesis that SNHL pathology is limited to the inner ear and is not situated in the cochlear nerve or brain stem. ${ }^{23,24}$ We could not report any data on inner ear pathology. However, MRIs of the cerebellopontine angle did not show any abnormalities in the anatomy, arguing against gross anatomical abnormalities of the inner ear. Future research focusing on inner ear changes, for instance with otoacoustic emissions and advanced electrophysiological measurements could possibly help elucidate the pathophysiology of SNHL in MPS. 
The MPS are rare diseases, therefore studying them inevitably results in a small number of patients, which is a limitation of this study. Also, because transplantation is performed in children at a very young age hearing assessments determining the exact hearing level before HCT were missing which hindered comparison pre- and post-intervention. Due to the longitudinal aspect of the study, however, we were able to show the course of hearing loss over time after HCT. Finally, as this was a retrospective study, we only had data collected from care-as-usual.

Concluding, hearing loss is still seen after long-term follow-up in MPS children despite HCT at an early age. When looking in-depth with a maximum follow-up of 16 years, air conduction is initially improving after HCT. Bone conduction however is slowly worsening over time, which could be a sign of disease progression in HCT-treated MPS patients. To improve the quality of life of these patients, new therapies should focus on how to stop post-transplant disease progression. Until then, MPS patients awaiting HCT should be informed that most likely ventilation tubes and/or hearing aids may be necessary at some point. Furthermore, after HCT, yearly evaluation with a hearing assessment by tone audiometry and potentially otoacoustic emissions is indicated for early diagnosis of the severity and type of hearing loss and to initiate treatment of hearing loss to ensure the requirements for adequate speech and language development and academic performance in these patients.

\section{ACKNOWLEDGEMENTS}

All authors would like to thank all parents and patients for participating in this study. 


\section{REFERENCES}

1. Ruckenstein MJ, Macdonald RE, Clarke JT, Forte V. The management of otolaryngological problems in the mucopolysaccharidoses: a retrospective review. $J$ Otolaryngol. 1991; 20: 177-183.

2. Simmons MA, Bruce IA, Penney S, Wraith E, Rothera MP. Otorhinolaryngological manifestations of the mucopolysaccharidoses. Int J Pediatr Otorhinolaryngol. 2005; 69: 589-595.

3. Keilmann A, Nakarat T, Bruce IA, Molter D, Malm G. Hearing loss in patients with mucopolysaccharidosis II: data from HOS - the Hunter Outcome Survey. J Inherit Metab Dis. 2012; 35: 343-353.

4. Lin H-Y, Shih S-C, Chuang C-K, et al. Assessment of hearing loss by pure-tone audiometry in patients with mucopolysaccharidoses. Mol Genet Metab. 2014; 111: 533-538.

5. Gokdogan C, Altinyay S, Gokdogan O, et al. Audiologic evaluations of children with mucopolysaccharidosis. Braz J Otorhinolaryngol. 2016; 82: 281-284.

6. Silveira MRM da, Buriti AKL, Martins AM, Gil D, Azevedo MF de. Audiometric evaluation in individuals with mucopolysaccharidosis. Clinics (Sao Paulo). 2018; 73: e523.

7. Ahn J, Lee JJ, Park S-I, et al. Auditory Characteristics in Patients With Mucopolysaccharidosis. Otol Neurotol Off Publ Am Otol Soc Am Neurotol Soc [and] Eur Acad Otol Neurotol. 2019; 40: e955-e961.

8. Muenzer J. Overview of the mucopolysaccharidoses. Rheumatology. 2011;50:v4-v12.

9. Bianchi PM, Gaini R, Vitale S. ENT and mucopolysaccharidoses. Ital J Pediatr. 2018; 44: 127.

10. Zechner G, Moser M. Otosclerosis and mucopolysaccharidosis. Acta Otolaryngol. 1987; 103: 384-386.

11. Gorlin RJ, Gorlin RJ, Toriello H V, Cohen MM. Hereditary Hearing Loss and Its Syndromes. Oxford University Press, USA; 1995.

12. Friedmann I, Spellacy E, Crow J, Watts RW. Histopathological studies of the temporal bones in Hurler's disease [mucopolysaccharidosis (MPS) IH]. J Laryngol Otol. 1985; 99: 29-41.

13. Tan EY, Boelens JJ, Jones SA, Wynn RF. Haematopoietic Stem Cell Transplantation in Inborn Errors of Metabolism-On Behalf of IEWP-EBMT. Front Pediatr. 2019; 7: 433.

14. Aldenhoven M, Jones SA, Bonney D, et al. Hematopoietic Cell Transplantation for Mucopolysaccharidosis Patients Is Safe and Effective: Results after Implementation of International Guidelines. Biol Blood Marrow Transplant. 2015; 21: 1106-1109. 
15. Aldenhoven M, Wynn RF, Orchard PJ, et al. Long-term outcome of Hurler syndrome patients after hematopoietic cell transplantation: an international multicenter study. Blood. 2015; 125: 2164-2172.

16. van den Broek BTA, van Egmond-Ebbeling MB, Achterberg JA, et al. Longitudinal analysis of ocular disease in children with Mucopolysaccharidosis I after hematopoietic cell transplantation. Biol Blood Marrow Transplant. 2019; Epub.

17. Papsin BC, Vellodi A, Bailey CM, Ratcliffe PC, Leighton SE. Otologic and laryngologic manifestations of mucopolysaccharidoses after bone marrow transplantation. Otolaryngol Head Neck Surg. 1998; 118: 30-36.

18. Da Costa V, O’Grady G, Jackson L, Kaylie D, Raynor E. Improvements in sensorineural hearing loss after cord blood transplant in patients with mucopolysaccharidosis. Arch Otolaryngol Head Neck Surg. 2012;138(11):10711076. doi:10.1001/jamaoto.2013.597

19. Deafness WHOP for the P of B and. WHO ear and hearing disorders survey. https:// apps.who.int/iris/handle/10665/67892.

20. Mazzoli M, Van Camp GUY, Newton V, Giarbini N, Declau F, Parving A. Recommendations for the description of genetic and audiological data for families with nonsyndromic hereditary hearing impairment. Audiol Med. 2003; 1: 148-150.

21. Boelens JJ, Rocha V, Aldenhoven M, et al. Risk factor analysis of outcomes after unrelated cord blood transplantation in patients with hurler syndrome. Biol Blood Marrow Transplant. 2009; 15: 618-625.

22. Aldenhoven M, A van den BBT, Wynn RF, et al. Quality of life of Hurler syndrome patients after successful hematopoietic stem cell transplantation. Blood Adv. 2017; 1: 2236-2242.

23. Saeed H, Nichani J, Melling C, et al. Feasibility of cochlear implantation in Mucopolysaccharidosis. Int J Pediatr Otorhinolaryngol. 2013; 77: 1255-1258.

24. Nagao K, Walter C, Parkes WJ, et al. Cochlear implantation in a patient with mucopolysaccharidosis IVA. SAGE open Med case reports. 2019; 7.

25. Kariya S, Schachern PA, Nishizaki K, Paparella MM, Cureoglu S. Inner ear changes in mucopolysaccharidosis type I/Hurler syndrome. Otol Neurotol Off Publ Am Otol Soc Am Neurotol Soc [and] Eur Acad Otol Neurotol. 2012;3 3: 1323-1327.

26. Schachern PA, Cureoglu S, Tsuprun V, Paparella MM, Whitley CB. Age-related functional and histopathological changes of the ear in the MPS I mouse. Int J Pediatr Otorhinolaryngol. 2007; 71: 197-203.

27. Hordeaux J, Deniaud J, Bemelmans I, et al. Histopathologic changes of the ear in canine models of mucopolysaccharidosis types I and VII. Vet Pathol. 2011; 48:616-626. 


\section{SUPPLEMENTAL FIGURES}
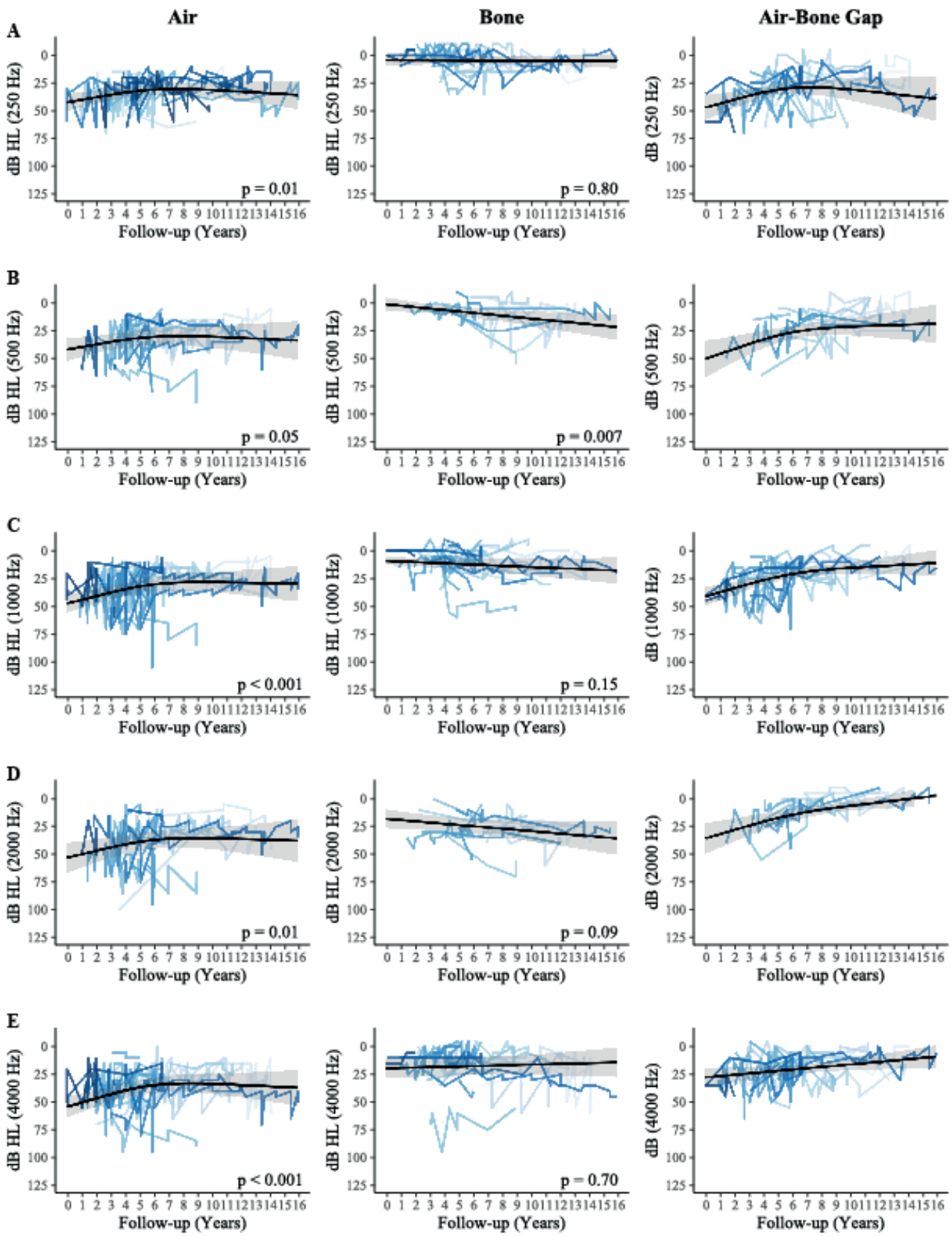

Suppl. figure 1. Air conduction, bone conduction and air-bone gap per individual patient over time (blue lines) for the different threshold test frequencies (A: $250 \mathrm{~Hz}, \mathrm{~B}: 500 \mathrm{~Hz}, \mathrm{C}: 1000 \mathrm{~Hz}, \mathrm{D}: 2000 \mathrm{~Hz}, \mathrm{E}$ : $4000 \mathrm{~Hz}$ ). The black line reflects the average course over time. 
Hearing loss in MPS after HCT $\mid 81$ 


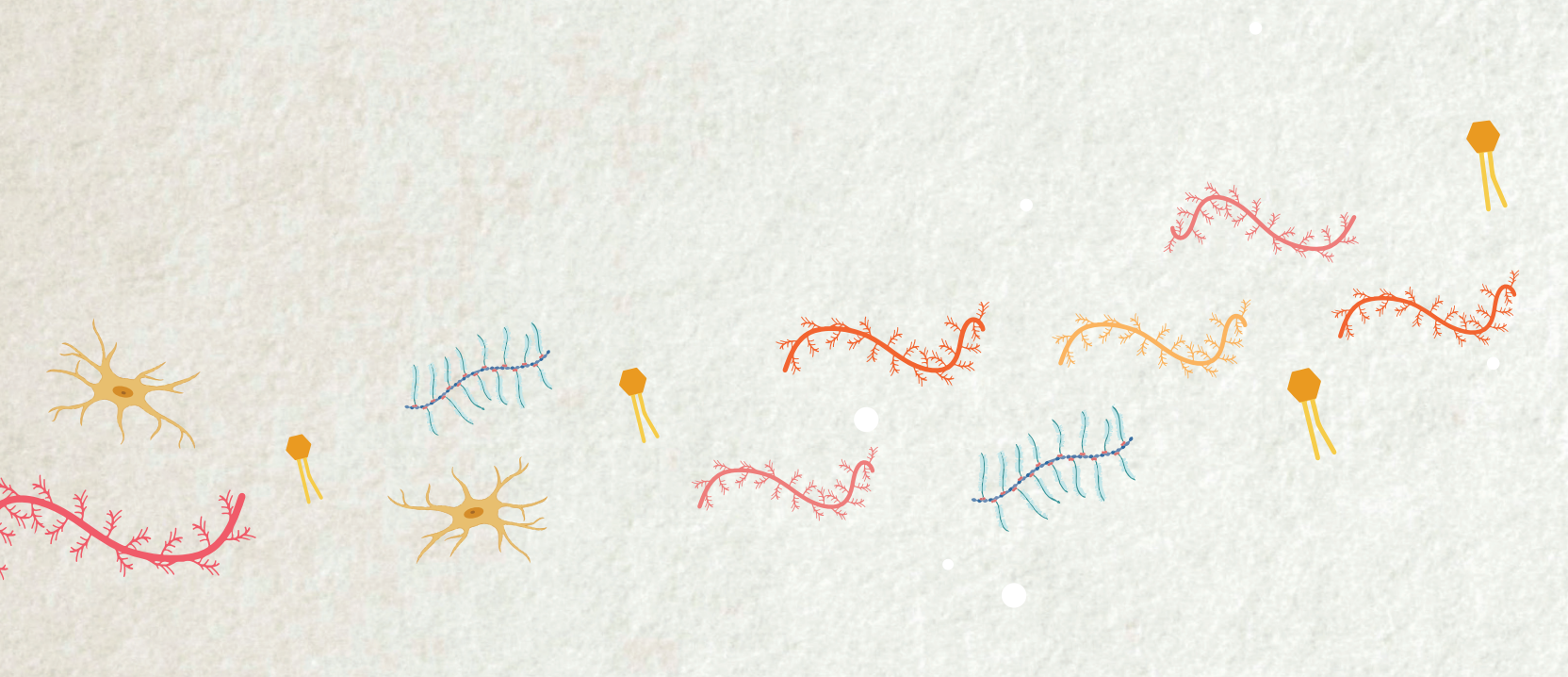



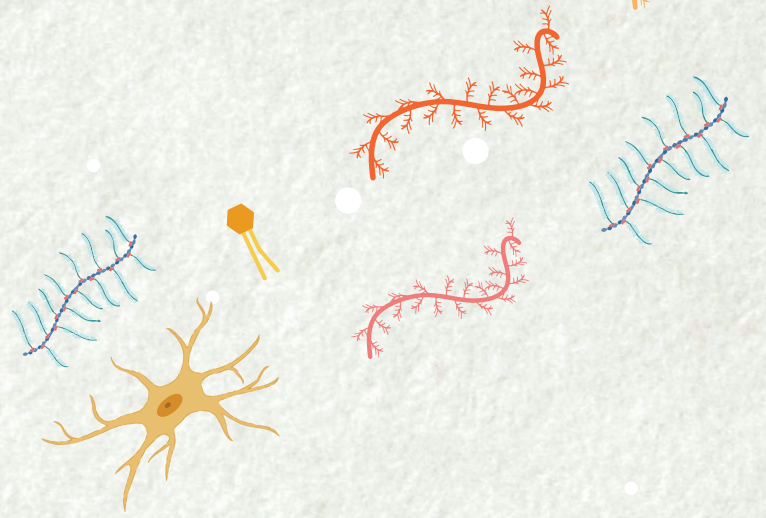

\section{CHAPTER 5}

\section{Quality of Life of Hurler Syndrome \\ Patients After Successful Hematopoietic \\ Cell transplantation}

Brigitte T.A. van den Broek*

Mieke Aldenhoven*

Robert F. Wynn

Anne O'Meara

Paul Veys

Attilio Rovelli

Simon A. Jones

*Contributed equally
Rossella Parini

Peter M. van Hasselt

Marleen Renard

Victoria Bordon

Tom J. de Koning

Jaap J. Boelens 


\begin{abstract}
Hurler syndrome (HS) is a lysosomal storage disease characterized by multi-system morbidity and death in early childhood. Hematopoietic cell transplantation (HCT) results in long-term survival, although with significant residual disease burden. How this residual disease affects the health-related quality of life is unknown. Therefore, we conducted a multi-center cohort study on functional and psychosocial health and compared the outcomes to normative data using the Child Health Questionnaire and Paediatric Outcomes Data Collection Instrument. Perception of care was evaluated by the Measure of Processes of Care questionnaire. Sixty-three HS patients receiving HCT with at least 3 years of follow-up after HCT were included. The influence of potential predictors was analyzed using linear regression analysis and correlation analysis was performed using Spearman rank correlation. Functional health of transplanted HS patients was significantly diminished compared with normative data: median physical summary z-score -2.4 (range $-3.5 ;-1.6$ ), median global functioning z-score -3.2 (range -4.8;-1.8). Psychosocial health was comparable or only slightly reduced compared to healthy peers: median psychosocial summary z-score 0.15 (range $-0.7 ; 0.8$ ). A higher obtained lysosomal enzyme level post-HCT predicted for superior functional health. Parents were overall satisfied with the care received. Functional health of transplanted HS patients appeared significantly more affected than psychosocial health. To improve functional health, the use of only non-carrier donors and striving to achieve full-donor chimerism, both resulting in higher enzyme levels, is advised. Assessing the health-related quality of life could play an important role in evaluating outcomes of HS patients receiving novel (cell-) therapies including autologous gene-transduced HCT.
\end{abstract}




\section{INTRODUCTION}

Hurler syndrome (HS) is a lysosomal storage disorder caused by a deficiency of the lysosomal enzyme alpha-L-iduronidase (IDUA). Without hematopoietic cell transplantation (HCT), this devastating disease is characterized by progressive multi-system morbidity including severe orthopedic complications, neurodevelopmental deterioration, and cardiopulmonary complications leading to death in early childhood (median survival 7.8 years). ${ }^{1-2}$ With more than 500 HCT procedures so far, HS is the most extensively transplanted inborn error of metabolism and therefore often serves as a paradigm disorder for HCT in these conditions. Although enzyme replacement therapy (ERT) has become available for Mucopolysaccharidosis type I, HCT remains the preferred treatment option in HS patients since it is the only treatment that delivers the deficient enzyme to the central nervous system necessary to prevent progressive neurodegeneration.

HCT dramatically attenuates the clinical course of HS and allows affected individuals to achieve long-term survival. However, significant residual disease burden is observed in almost all transplanted patients, with striking variability between patients. ${ }^{3}$ One of the main issues are the orthopedic complications, requiring surgical intervention in the majority of the patients despite successful HCT. ${ }^{4}$ To what extent this disease burden impacts on the healthrelated quality of life of transplanted HS patients remains unknown. Furthermore, due to the large number of complications that can be encountered in the years following HCT, all patients require long-term care from a large number of medical specialists. Until now, little is known about the parents' perception of care of transplanted HS patients.

This multi-center cohort study was conducted to provide a broader context of the burden of disease of HS patients, addressing the health-related quality of life as well as the processes of care of transplanted HS patients from parents' perspective.

\section{PATIENTS AND METHODS}

\section{Inclusion criteria}

HS patients receiving an allogeneic HCT in one of the seven participating European transplant centers of expertise and present during a multidisciplinary follow-up day at the local center were evaluated for inclusion in the study. Assays of leukocyte IDUA activity at presentation in combination with the clinical phenotype confirmed the diagnosis in all patients. For inclusion, patients had to be between 5 and 18 years of age with more than $10 \%$ 
donor chimerism and a minimum of 3 years follow-up post-HCT. Only patients with at least one completed questionnaire were included.

\section{Data collection}

One member of the study team (M.A.) visited all participating study centers during multidisciplinary follow-up days. The parents of eligible patients were asked to complete three questionnaires, described in more detail below. The medical records of all included patients were retrospectively evaluated, according to a standardized set of potential patient, donor, transplantation, and disease-related predictors. Cognitive developmental impairment (IQ $<70$ ), growth retardation ( $<-2 \mathrm{SD}$, according to the WHO growth curves), ${ }^{5}$ and in receipt of medical intervention at time of transplant were considered potential disease-related predictors.

This study was approved by the institutional review boards of the University Medical Center Utrecht (center of principal investigator) and all other participating centers thereafter. Written informed consent was obtained from the parents or legal guardians of the patients.

\section{Questionnaires}

Child Health Questionnaire: The 28-item parent form of the Child Health Questionnaire (CHQ-PF28) is a validated questionnaire, designed and normed for children aged 5-18 years. ${ }^{6}$ All subscale scores were generated using the scoring algorithm provided by the authors of the instrument. The various subscale scores were aggregated as appropriate to derive two summary component scores: the physical and psychosocial summary scores. The subscale and summary scores were compared to a normative sample to derive z-scores. ${ }^{6}$

Pediatric Outcomes Data Collection Instrument: The Pediatric Outcomes Data Collection Instrument (PODCI) is a validated questionnaire designed by the American Academy of Orthopedic Surgeons and the Pediatric Orthopedic Society of North America for children 2-18 years of age with moderate to severe orthopedic disease. ${ }^{7}$ All subscale scores were generated using the scoring algorithm provided by the authors of the instrument. A global functioning score was calculated, combining four of the subscales and z-scores were derived by comparison to a normative sample. ${ }^{8}$

Measure of Processes of Care questionnaire: The 20-item Measure of Processes of Care (MPOC-20) questionnaire was used to assess the parents' perceptions of the care they receive from their treatment center. This questionnaire is validated for children aged 0-18 years. ${ }^{9-10} \mathrm{All}$ scores were generated using the scoring algorithm provided by the authors of the instrument.

\section{Endpoints}

Functional health: Functional health domains assessed using the PODCI included the 'upper extremity function', 'transfers and mobility', 'sports and physical function', and 'pain and 
comfort' subscales as well as the 'global functioning' summary score. The items of the CHQ attributing to the functional health were the 'physical functioning', 'role/social limitationsphysical', 'general health', 'bodily pain', and 'parental impact-time' subscales as well as the 'physical summary score'.

Psychosocial health: Psychosocial health domains assessed using the PODCI included the 'pain and comfort' and 'happiness' subscales as well as the 'global functioning' summary score. Regarding the CHQ the 'role/social limitations-emotional/behavioral', 'general health', 'bodily pain', 'parental impact-emotional', 'self-esteem', 'mental health', and 'behavior' subscales as well as the 'psychosocial summary score' were attributed to the psychosocial health.

Perception of care: Perception of care was assessed using the five subscales of the MPOC questionnaire with scores ranging from 1 ('not at all') to 7 ('to a very great extent').

\section{Statistical analysis}

To identify differences concerning patient, donor, transplantation, and disease-related characteristics between the eligible patients included in the study and those eligible but not included, Chi square and ANOVA tests were used. The association between the various potential patient, donor, transplantation, and disease-related predictors mentioned in table 1 and the endpoints were analyzed using univariate and multivariate linear regression analysis. Univariate predictors of outcome parameters that were statistically significant $(P<0.10)$ were selected for multivariate analysis. Results were expressed as odds ratios and corresponding $95 \%$ confidence intervals. $P$ values $<0.05$ were considered statistically significant. Spearman rank correlations, expressed as a correlation coefficient (r), were used to compare correlations between the individual functional and psychosocial PODCI and CHQ subscale and summary scores. Statistical analysis was performed using SPSS 20.0 (SPSS Inc., Chicago, IL) and RStudio 0.99.441 (RStudio Inc., Boston, MA). Graphs were created using RStudio 0.99.441.

\section{RESULTS}

\section{Study population}

The questionnaires were distributed amongst the parents of 81 eligible patients of whom 63 (78\%) completed at least one of the questionnaires. The inability of parents to complete the questionnaires due to language barriers was the main reason not to be included in the study.

The included Hurler patients were transplanted at a median age of 15 months (range 3-42). The median age at assessment was 8 years (range 5-18). The stem cell source consisted of a 
bone marrow (63\%), unrelated cord blood (24\%) or peripheral blood stem cell (13\%) graft. Thirty-two percent of the patients obtained enzyme levels below the local lower reference limit, due to mixed-donor chimerism (in 24\%) and/or the use of a carrier donor (19\%).

Table 1. Patient, donor, and transplantation-related characteristics.

\begin{tabular}{lcc}
\hline Patient-related characteristics & N (\%) & Median (range) \\
\hline Overall & $34(54)$ & $63(97)$ \\
Gender (male) & $28(47)$ & $15(3-42)$ \\
Ethnicity (Caucasian) & & $6(0-27)$ \\
Genotype (nonsense-nonsense*) & & $8(5-18)$
\end{tabular}

\begin{tabular}{lcc}
\hline Donor-related characteristics & N $(\%)$ & Median (range) \\
\hline Source (CB/BM/PBSC) & $15 / 40 / 8(24 / 63 / 13)$ \\
Relation (related) & $23(37)$ \\
Carrier status (carrier) & $11(19)$
\end{tabular}

\begin{tabular}{lcc}
\hline Transplantation-related characteristics & N (\%) & Median (range) \\
\hline No of HCT (1/2/3) & $46 / 16 / 1(73 / 25 / 2)$ & $2002(1987-2009)$ \\
Year of HCT & $20(32)$ & $2(3)$ \\
ERT pre-HCT & $15(24)$ \\
TBI & $20(32)$ \\
Donor chimerism (<95\%) & \\
IDUA level post-HCT $\dagger(<$ reference $\$)$ & $59(13-190)$ \\
IDUA level post-HCT $\dagger(\%$ of mean)
\end{tabular}

All characteristics concern the last HCT. BM indicates bone marrow; CB, cord blood; ERT, enzyme replacement therapy; HCT, hematopoietic cell transplantation; IDUA, alpha-L-iduronidase; mo, months; n, number; PBSC, peripheral blood stem cells; TBI, total body irradiation; and yr, years.

*of known mutations, †measured in leukocytes, ‡lower limit of local reference. 
Enzyme levels obtained $\geq 1$ year after HCT showed minimal change over time. The patient, donor, and transplantation-related characteristics of the 63 included patients are shown in Table 1. Only ethnicity $(P=0.008)$ and relationship of donor $(P=0.02)$ differed between the eligible patients included in the study and those not included (data not shown).

Of the included patients, $46 \%$ suffered from cognitive developmental impairment, while $51 \%$ had growth retardation. Medical interventions were frequently performed in the patients included in this study. Data regarding the disease-related characteristics, including cognitive development, growth, and the various medical interventions are shown in Table $\mathrm{S} 1$. No significant differences in cognitive development, growth, or occurrence of a medical intervention were found between the included patients and those not included in the study (data not shown). Of the 63 included patients in the study, the CHQ, PODCI, and MPOC questionnaire were completed in $92 \%, 90 \%$, and $97 \%$.

\section{Health-related quality of life - Functional health}

Significantly reduced scores were observed regarding the functional health subscales of both the CHQ and PODCI when compared with normative data. This is also demonstrated by the low summary scores in both questionnaires; median physical summary z-score -2.4 (range -3.5;-1.6) and mean global functioning z-score -3.2 (range -4.8;-1.8) (Figure 1-2). The reduced summary scores were largely determined by the low 'physical functioning' subscale of the CHQ and the 'transfer \& basic mobility' and 'sports \& physical function' subscales of the PODCI.

\section{Health-related quality of life - Psychosocial health}

The psychosocial health appeared to be normal to only slightly reduced compared to normative data, demonstrated by the psychosocial summary score of the CHQ; median 0.15 (range -0.7;0.8). The 'self-esteem', 'mental health', and 'behavior' subscales of the CHQ showed to be comparable to the healthy population sample (Figure 1).

Table 2. Multivariate predictor analysis

\begin{tabular}{lcccccc}
\hline Questionnaire & Summary score & $\begin{array}{c}\text { Median z-score } \\
\text { (range) }\end{array}$ & Predictor & Cut-off & $\begin{array}{c}\text { OR } \\
\text { (95\% CI) }\end{array}$ & P \\
\hline CHQ & Physical & $-2.4(-3.5 ;-1.6)$ & $\begin{array}{c}\text { IDUA level } \\
(\% \text { of mean })\end{array}$ & Median & $\begin{array}{c}3.02(0.01- \\
0.02)\end{array}$ & 0.004 \\
& Psychosocial & $0.15(-0.7 ; 0.8)$ & & & 2.01 & 0.049 \\
PODCI & $\begin{array}{l}\text { Global } \\
\text { Functioning }\end{array}$ & $-3.2(-4.8 ;-1.8)$ & $\begin{array}{c}\text { IDUA level } \\
(\% \text { of mean })\end{array}$ & Median & $(0.00-0.03)$ & \\
\hline
\end{tabular}

Only statistically significant results are shown. CHQ indicates Child Health Questionnaire; CI, confidence interval; OR, Odds ratio; P, p-value; and PODCI, Pediatric Outcomes Data Collection Instrument. 


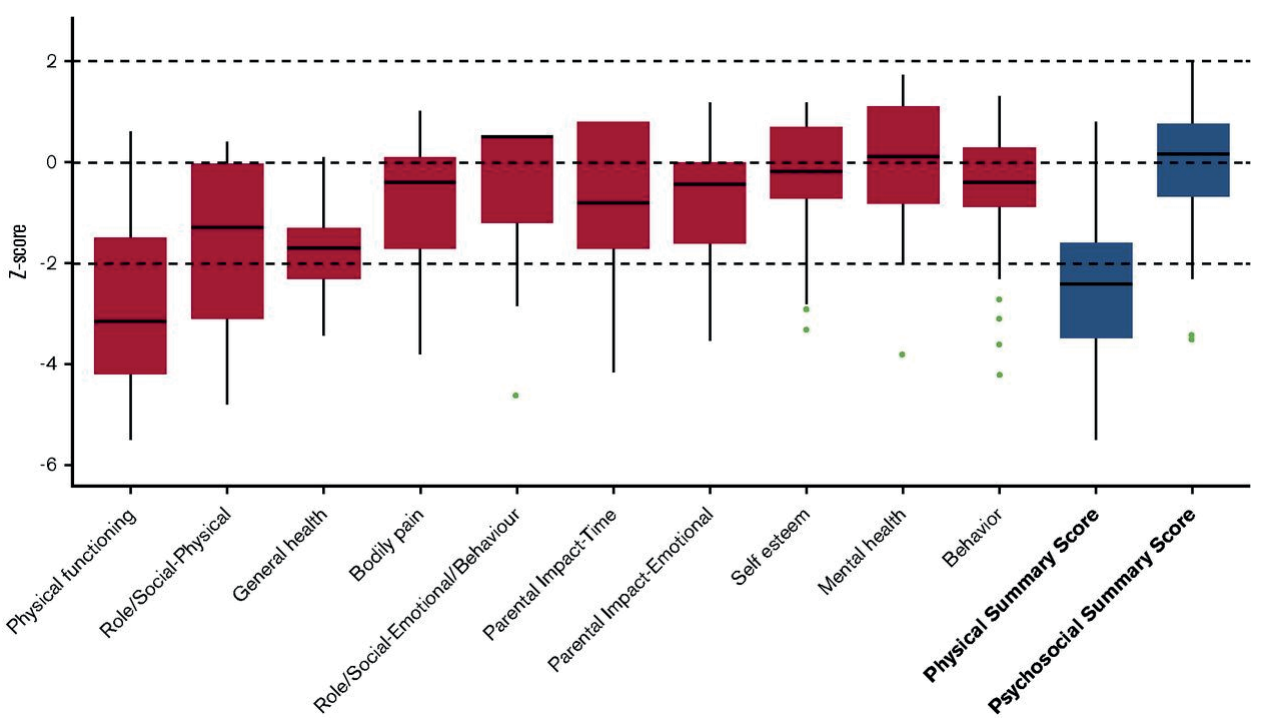

Figure 1. Child Health Questionnaire (CHQ). Boxplot showing the individual z-scores, with the median and interquartile range. The results of the subscales are depicted in white, those of the summary scores in grey.

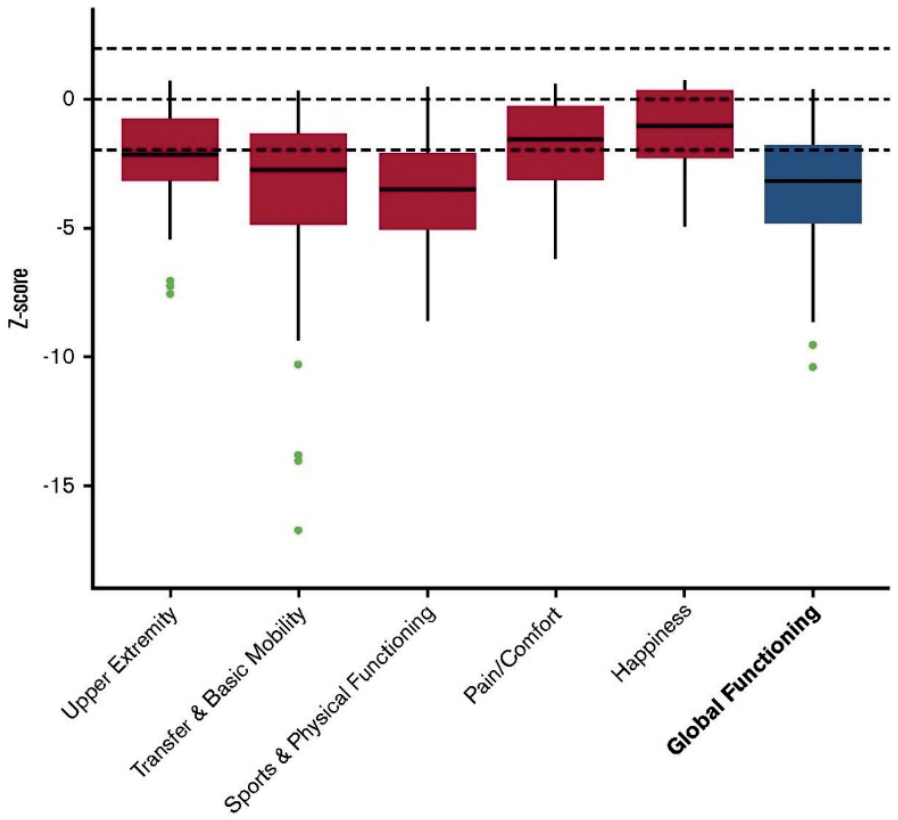

Figure 2. Pediatric Outcomes Data Collection Instrument (PODCI). Boxplot showing the individual $\mathrm{z}$-scores, with the median and interquartile range. The results of the subscales are depicted in white, the summary score is depicted in grey. 


\section{Health-related quality of life - Predictors}

A lower obtained IDUA enzyme level, expressed as $\%$ of the mean, predicted for a significantly lower functional health concerning both the CHQ and PODCI; physical summary score, $P=0.004$, global functioning score $P<0.001$ (Table 2). No significant association was found between any of the other potential patient, donor, transplant, or disease-related predictors and the various functional or psychosocial health scores.

\section{Health-related quality of life - Correlation between CHQ and PODCI}

The scores of all functional domains in the CHQ did significantly correlate with those in the PODCI, except for 'bodily pain' in the CHQ and 'upper extremity' in the PODCI (Table S2). The physical summary score of the CHQ and the global functioning scale of the PODCI showed excellent correlation $(r=0.772, P<0.001)$. Regarding psychosocial function, the scores of 'general health', 'bodily pain', and 'mental health' in the CHQ did highly correlate with all psychosocial domains in the PODCI, except for that of 'general health' and 'happiness'.

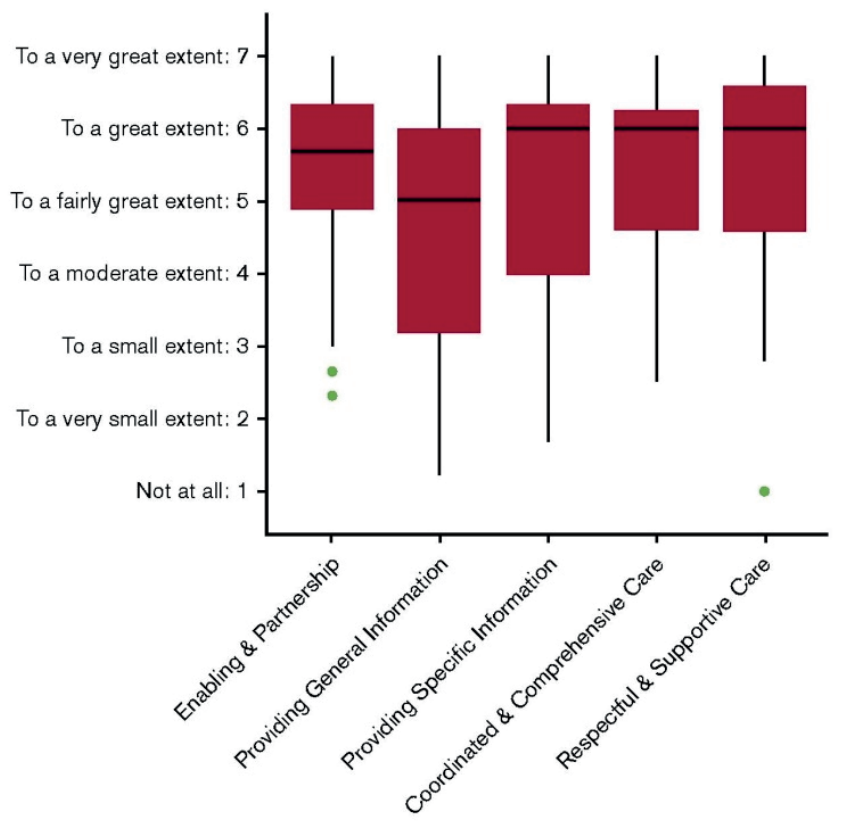

Figure 3. Measure of Processes of Care (MPOC) questionnaire.

Boxplot showing the individual scores, with the median and interquartile range. 


\section{Processes of Care}

The parents were overall satisfied with the care they received from the transplantation center, with median scores ranging from 5.0 ('providing general information') to 6.0 ('respectful and supportive care') (Figure 3).

\section{DISCUSSION}

This cross-sectional survey confirms that the functional health of HS patients post-HCT is significantly diminished compared to a normative population. This is presumably due to the residual disease burden observed in transplanted patients, especially the frequently occurring orthopedic complications. The psychosocial health showed a much more favorable outcome with normal or only slightly reduced scores compared to their healthy peers.

Due to the collaborative effort, a substantial population size was achieved despite the relative rarity of the disease. Although there was a large age range of the patients assessed, this did not have a significant impact on any of the functional or psychosocial health measures. In previous research, age was mainly found a predictor for neurocognitive outcomes, which was not a studied outcome in this study. ${ }^{4}$ Interestingly, none of the disease-related characteristics was found to be a predictor for either functional or psychosocial health. This was similar for patients receiving ERT prior to HCT. The only predictor found in this study was the IDUA enzyme level obtained post-HCT, significantly influencing functional health. Previously, this same predictor also appeared to be a significant predictor for the prognosis in HS patients after HCT, including orthopedic outcome measures. ${ }^{4}$ Superior functional health might therefore be achieved in this patient population if higher IDUA enzyme levels are obtained post-HCT, although in this study as in a previous study we did not find a threshold effect in the normal IDUA level range. ${ }^{4}$ Higher IDUA levels can be realized by using exclusively noncarrier donors and transplantation techniques promoting complete donor chimerism. Previous studies have showed that the chance at full donor chimerism is higher after cord blood transplantation. ${ }^{11}$ Our analyses did not find CB to be a predictor for better outcomes, which might be due to a relatively low number $(n=15)$ of cord blood transplantations. Whether supra-normal enzyme levels, potentially obtained by new treatment approaches such as autologous gene-transduced HCT resulting in overexpression of the enzyme, will improve the functional health even more remains an interesting question and needs to be elucidated. ${ }^{12}$ The first trials of gene therapy in HS patients are close to clinic.

A potential limitation in our evaluation of functional and psychosocial health in HS patients was the fact that these outcome measures were evaluated by their parents. Most of our 
included patients were too young to complete the questionnaires or lacked the necessary cognitive abilities to effectively interpret and answer the questions. Therefore, the number of patient reports that could potentially be used in the analysis was too low and only parent forms were included. Interestingly, a previous study in children with a wide range of musculoskeletal problems showed an overall strong level of agreement between the child and parent responses for most domains in both the CHQ and PODCI, although parents had a tendency to report more physical limitations and pain than these children acknowledged themselves. ${ }^{13}$ It is therefore likely that the responses of the parents in this study reliably represent that of their children as well. Nevertheless, it would be of great interest and importance to continue to evaluate the functional and psychosocial health in this group of patients in upcoming decade(s).

Although assessment of the various organ systems by medical specialists during followup is an important aspect in evaluating treatment outcome, we believe that this should be accompanied by evaluation of the health-related quality of life from the patients' or parents' perspective. Furthermore, since the residual burden of the disease has a complex effect on health status, including the physical as well as psychosocial health, it is important to assess both health modalities. This might help to identify specific areas of difficulties these patients might encounter, enabling the most appropriate support. Importantly, there appeared to be a modest to strong correlation between the various functional and psychosocial domain scores of the CHQ and PODCI questionnaires, indicating that both questionnaires comparably measure these outcomes in this patient population. The CHQ offers a broader scope of health and can therefore examine functional as well as psychosocial attributes. The PODCI, however, offers a more detailed picture of physical functioning, with the possibility to differentiate between the various physical problems. ${ }^{14-15}$

The findings of the MPOC questionnaire indicate that parents of patients with HS patients overall positively rate the care they receive. Most improvement can be achieved by addressing the relatively low scores on the provision of general information. All centers participating in this study have developed multidisciplinary settings with highly experienced specialists to evaluate these patients and provide medical intervention where required. This might have been contributed to the high level of satisfaction observed in this study.

Taken together, the functional health of transplanted HS patients was significantly more affected than the psychosocial health, both demonstrated by the CHQ and PODCI. A higher obtained IDUA enzyme level after HCT predicted for superior functional health, supporting the use of only non-carrier donors and striving to achieve full-donor chimerism. Assessing the functional and psychosocial health should play an important role in the evaluation of 
outcomes in HS patients receiving HCT as well as upcoming new treatment approaches, such as autologous gene-transduced HCT aiming for overexpression of the enzyme. Furthermore, the parents were overall satisfied with the care provided. Multidisciplinary settings involving specialists experienced in HS for long-term follow-up and medical interventions are recommended.

\section{ACKNOWLEDGEMENTS}

The authors thank all parents and patients for completion of the questionnaires and all participating centers for sharing patient information. 


\section{REFERENCES:}

1. Neufeld EF, Muenzer J. The Mucopolysaccharidosis. In: Scriver C, Beaudet A, Sly W, Valle D, eds. The Metabolic and Molecular Basis of Inherited Disease. New York, NY: McGraw-Hill; 2001: 3421-3452.

2. Moore D, Connock MJ, Wraith E, Lavery C. The prevalence of and survival in Mucopolysaccharidosis I: Hurler, Hurler-Scheie and Scheie syndromes in the UK. Orphanet J Rare Dis. 2008; 3: 24.

3. Aldenhoven M, Boelens JJ, de Koning TJ. The clinical outcome of Hurler syndrome after stem cell transplantation. Biol Blood Marrow Transplant. 2008; 14: 485-498.

4. Aldenhoven M, Wynn RF, Orchard PJ, et al. Predictors of Long-term Clinical Outcome in Hurler Syndrome Patients after Successful Hematopoietic stem cell transplantation: an International, Multi-Center Study. Blood. 2015; 125: 2164-2172.

5. World Health Organization. Child Growth Standards 2006. http://www.who.int/ childgrowth. Accessed September, 2014.

6. Landgraf JM, Abetz L, Ware JE. The CHQ user's manual. Boston, MA: The Health Institute, New England Medical Center. 1996.

7. Daltroy LH, Liang MH, Fossel AH, Goldberg MJ. The POSNA pediatric musculoskeletal functional health questionnaire: report on reliability, validity, and sensitivity to change. Pediatric Outcomes Instrument Development Group. Pediatric Orthopaedic Society of North America. J Pediatr Orthop. 1998; 18 :561-571.

8. Haynes RJ, Sullivan E. The Pediatric Orthopaedic Society of North America pediatric orthopaedic functional health questionnaire: an analysis of normals. $J$ Pediatr Orthop. 2001; 21: 619-621.

9. King S, King G, Rosenbaum P. Evaluating health service delivery to children with chronic conditions and their families: development of a refined Measure of Processes of Care (MPOC-20). Children's Health Care. 2004; 33: 35-57.

10. Siebes RC, Maassen GH, Wijnroks L, et al. Quality of paediatric rehabilitation from the parent perspective: validation of the short Measure of Processes of Care (MPOC-20) in the Netherlands. Clin Rehabil. 2007; 21:62-72.

11. Aldenhoven, M., \& Kurtzberg, J. Cord blood is the optimal graft source for the treatment of pediatric patients with lysosomal storage diseases: clinical outcomes and future directions. Cytotherapy. 2015; 17: 765-774.

12 Visigalli I, Delai S, Politi LS, et al. Gene therapy augments the efficacy of hematopoietic stem cell transplantation and fully corrects mucopolysaccharidosis type I phenotype in the mouse. Blood. 2010; 116: 5130-5139.

13. Matsumoto H, Vitale MG, Hyman JE, Roye DP Jr. Can parents rate their children's 
96 Chapter 5

quality of life? Perspectives on pediatric orthopedic outcomes. J Pediatr Orthop B. 2011; 20: 184-190.

14. Pencharz J, Young NL, Owen JL, Wright JG. Comparison of three outcomes instruments in children. J Pediatr Orthop. 2001; 21:425-432.

15. Vitale MG, Levy DE, Moskowitz AJ, et al. Capturing quality of life in pediatric orthopaedics: two recent measures compared. J Pediatr Orthop. 2001; 21: 629-635. 


\section{SUPPLEMENTAL TABLES}

Suppl. table 1. Cognitive development, growth, and medical interventions.

\begin{tabular}{|c|c|}
\hline Disease-related characteristics & $\mathbf{N}(\%)$ \\
\hline Cognitive developmental impairment (IQ <70) & $19(46)$ \\
\hline Growth retardation (height $<-2 \mathrm{SD}$ ) & $30(51)$ \\
\hline \multicolumn{2}{|l|}{ Neurologic intervention } \\
\hline Hydrocephalus - Ventriculo-peritoneal shunt & $7(11)$ \\
\hline \multicolumn{2}{|l|}{ Orthopedic intervention } \\
\hline Thoracolumbar kyphosis - Surgical intervention & $15(25)$ \\
\hline Cervical instability - Surgical intervention & $4(7)$ \\
\hline Cord compression - Surgical intervention & $4(7)$ \\
\hline Hip dysplasia - Surgical intervention & $22(36)$ \\
\hline Genu valgum - Surgical intervention & $19(31)$ \\
\hline Carpal tunnel syndrome - Surgical intervention & $25(41)$ \\
\hline Wheelchair dependence & $4(7)$ \\
\hline \multicolumn{2}{|l|}{ Cardiac intervention } \\
\hline Cardiomyopathy - ACE-inhibitor & $10(17)$ \\
\hline \multicolumn{2}{|l|}{ Respiratory intervention } \\
\hline Overnight hypoxia - Respiratory support & $3(5)$ \\
\hline \multicolumn{2}{|l|}{ Ophthalmologic intervention } \\
\hline Corneal clouding - Corneal transplant & $6(10)$ \\
\hline \multicolumn{2}{|l|}{ Audiologic intervention } \\
\hline Hearing loss - Hearing aids & $19(31)$ \\
\hline
\end{tabular}

ACE indicates Angiotensin-converting enzyme; IQ, intelligence quotient; n, number; and SD, standard deviation 


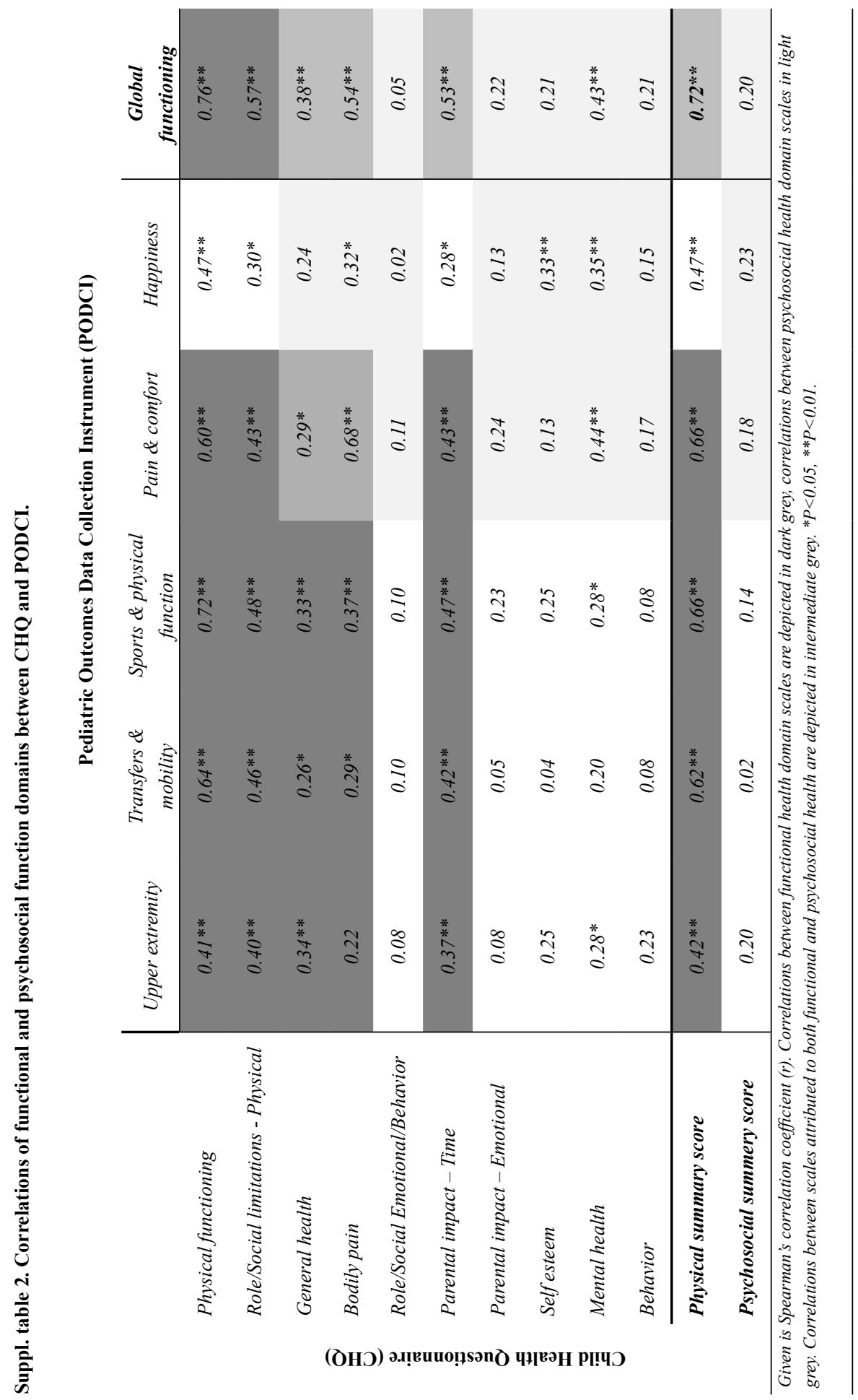


Quality of Life in MPS-1 after HCT $\mid 99$ 


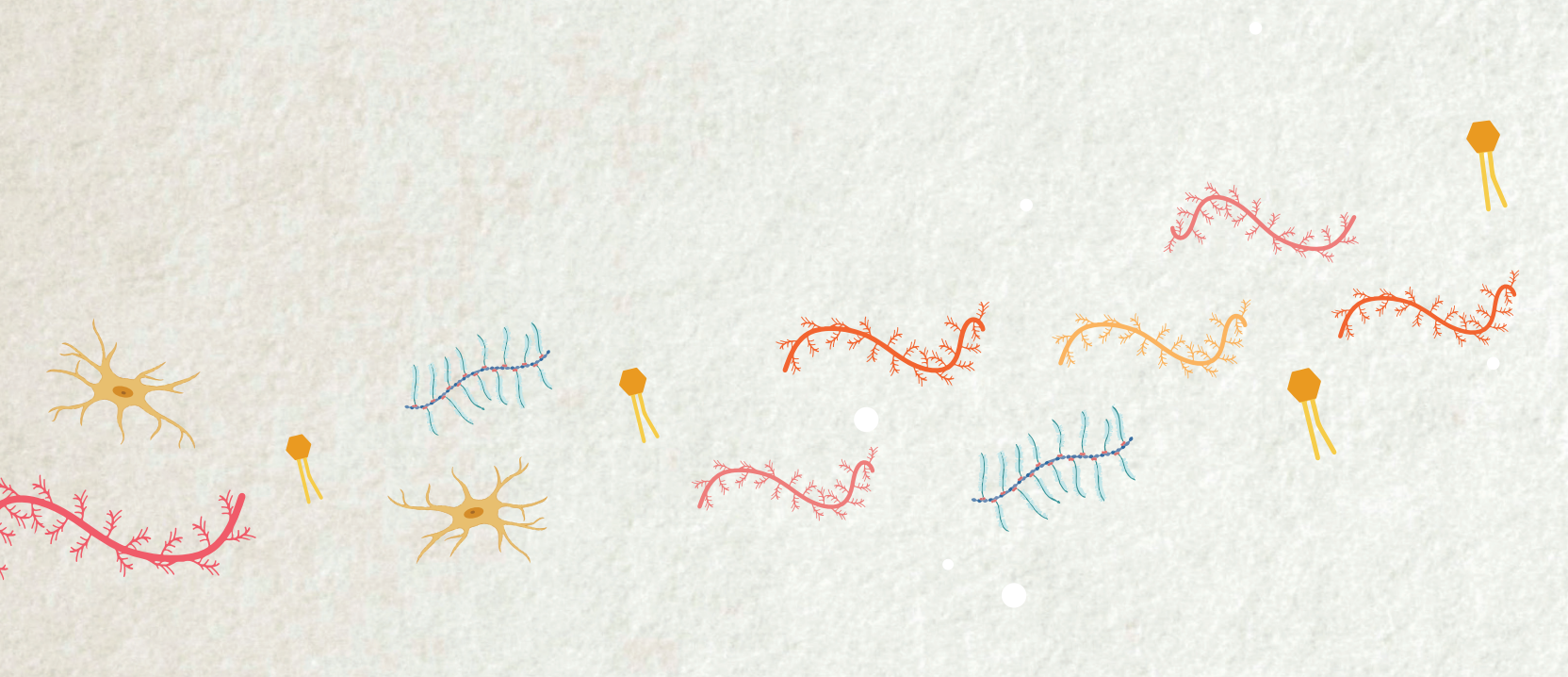



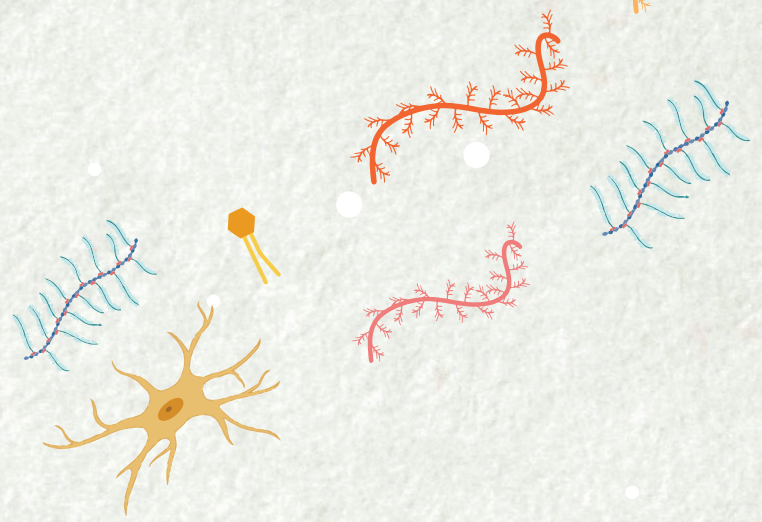

\section{CHAPTER 6}

How to Follow-up Mucopolysaccharidoses

Patients After Hematopoietic Cell

Transplantation?

Brigitte T.A. van den Broek

Mieke Aldenhoven

Klaas Koop

Peter M. van Hasselt

Jaap Jan Boelens 


\section{INTRODUCTION}

The Mucopolysaccharidoses (MPS) comprise a group of inborn errors of metabolism caused by specific lysosomal enzyme deficiencies, resulting in subsequent progressive accumulation of incompletely degraded glycosaminoglycans (GAGs). Although there is a wide spectrum of clinical manifestations varying among the different MPS disorders, a shared phenomenon is that most patients appear normal at birth followed by progressive multisystem deterioration. ${ }^{1}$ Since the first report of a MPS I-Hurler syndrome patient treated in $1981^{2}$, hematopoietic cell transplantation (HCT) has been performed extensively in MPS diseases, and its scope could be expanded due to the reduced rates of mortality and morbidity associated with the procedure. ${ }^{3,4}$ While enzyme replacement therapy (ERT) has become available for several MPS types, this treatment is incapable of crossing the blood-brain-barrier resulting in insufficient benefit for disorders associated with neurologic deterioration. Therefore, HCT is generally indicated in MPS diseases with central nervous system (CNS) involvement, although extensive CNS damage at time of presentation limits the effectiveness of HCT (table 1). ${ }^{4}$

Table 1. The Mucopolysaccharidoses - incidence, involved enzyme, and current HCT and gene therapy indication

\begin{tabular}{|c|c|c|c|c|c|c|}
\hline \multicolumn{3}{|c|}{ MPS (sub)type } & \multirow[t]{2}{*}{ Birth prevalence $^{a}$} & \multirow[t]{2}{*}{ Involved enzyme } & \multirow{2}{*}{$\begin{array}{l}\text { HCT } \\
\text { indication }^{\mathrm{b}} \\
\text { Standard } \\
\end{array}$} & \multirow{2}{*}{$\begin{array}{c}\text { Gene therapy } \\
\text { indication } \\
\text { Investigational }\end{array}$} \\
\hline I & Hurler & & & & & \\
\hline & Hurler-Scheie & & 1.19 per 100.000 & alpha-L-iduronidase & Option $^{\mathrm{c}}$ & \\
\hline & Scheie & & & & Option $^{\mathrm{c}}$ & \\
\hline II & Hunter & $\begin{array}{l}\text { type A } \\
\text { type B }\end{array}$ & 1.30 per $100.000^{\mathrm{d}}$ & Iduronate sulfatase & $\begin{array}{l}\text { Investigational }^{\mathrm{e}} \\
\text { Investigational }^{\mathrm{e}}\end{array}$ & Investigational \\
\hline \multirow[t]{4}{*}{ III } & Sanfilippo & type A & & Heparan N-sulfatase & Controversial & Investigational \\
\hline & & type B & & $\begin{array}{l}\mathrm{N} \text {-acetyl } \\
\text { glucosaminidase }\end{array}$ & Controversial & Investigational \\
\hline & & type $\mathrm{C}$ & 1.89 per 100.000 & $\begin{array}{l}\text { AcetylCoA:N- } \\
\text { acetyltransferase } \\
\text { N-acetyl }\end{array}$ & Controversial & \\
\hline & & type D & & $\begin{array}{l}\text { glucosamine } \\
\text { 6-sulfatase }\end{array}$ & Controversial & \\
\hline \multirow[t]{2}{*}{ IV } & Morquio & & 0.20 per 100.000 & $\begin{array}{l}\text { Galactosamine } \\
\text { 6-sulfatase }\end{array}$ & Option $^{\mathrm{c}}$ & \\
\hline & & type B & & beta-Galactosidase & Option $^{\mathrm{c}}$ & \\
\hline VI & Maroteaux-Lamy & & 0.15 per 100.000 & Arylsulfatase B & Option $^{\mathrm{c}}$ & Investigational \\
\hline VII & Sly & & 0.24 per 100.000 & beta-Glucuronidase & Option & \\
\hline
\end{tabular}

${ }^{\mathrm{a}}$ In the Netherlands, ${ }^{\mathrm{b}}$ adjusted from the EBMT guidelines[CIBMT working committee], ${ }^{\mathrm{c}}$ enzyme replacement therapy first line treatment, ${ }^{\mathrm{d}}$ males only (X-linked Disease), ${ }^{\mathrm{e}}$ only early and/or in asymptomatic patients. 
Although significant improvement in the clinical course of MPS patients has been achieved after HCT, disease progression is observed in all of them. ${ }^{5-8}$ Since it involves a group of rare diseases characterized by a broad range of clinical manifestations, and because patients are now alive decades after transplantation ${ }^{9}$ guidelines on the long term multidisciplinary care and follow-up of these patients is highly important. Multidisciplinary follow-up will help improve patient care, and follow-up data from these patient groups will also help evaluate the (added) benefit of future therapies. An overview of the evaluations at baseline and follow-up after HCT as well as their proposed frequency is shown in table 2 and further described below. These recommendations apply to the most frequently transplanted MPS types I-Hurler, II-Hunter, III-Sanfilippo, IV-Morquio, and VI-Maroteaux-Lamy. ${ }^{10}$ Since MPS I-Hurler syndrome accounts for the majority of HCT performed for MPS diseases, this disease often serves as a prototype. Finally, a clinical trial studying autologous gene-modified hematopoietic cell transplantation is currently open for MPS-1 and potentially will become the new standard treatment for some subtypes of MPS disorders. As the long-term effects are yet unknown, a systematic follow-up of these patients will still be required.

\section{Pre-transplant evaluation}

Pre-transplant evaluations should be performed not only to assess whether the patient

qualifies for the transplant and to determine disease specific issues that need addressing prior to transplant (particularly hydrocephalus) but also to create a baseline, essential for the correct interpretation of results post-transplant. Pre-transplant evaluation should be confirm the evaluation of the first 1-3 years after transplantation (table 2).

\section{Metabolic diseases}

Biochemical evaluation of the metabolic correction after HCT by regular analysis of the lysosomal enzyme activity in circulating mononuclear cells, especially during the first year after transplant, is recommended. After HCT, hematological enzyme activity should increase to values reflecting the status of the donor. ${ }^{3}$ For instance, if an unaffected donor is utilized and full engraftment is achieved, it would be expected that the enzyme activity in the circulation post-transplant would be normal. In contrast, if a matched related sibling who is a carrier for the disease served as the donor, the enzyme levels post-transplant would be expected to be approximately $50 \%$ of normal values. Similarly, if a graft was used from a homozygous unaffected donor, and incomplete chimerism is observed, the enzyme levels would we be expected to reflect this. Heterozygous donors are not recommended as studies have shown unfavorable outcomes in patients with reduced activity levels after HCT. Importantly, it should be recognized that the measurement of enzyme levels post-transplant reflects the concentration of enzyme in cell lysates of hematopoietic cells derived from the graft, and is not an indication of the enzyme level delivered to the liver, lung, heart, cornea, bone or brain. 
These determinations would be of interest, but are impractical as it would require tissue to be obtained from these sites. As a proxy for these measurements of enzyme activity in nonhematological compartments, measurements in saliva and tear fluid have been studied. ${ }^{11,12}$ MPS I patients post-transplantation still show very low enzyme activity levels in both saliva and tear fluid, indicating poor penetration in certain tissues. The levels do increase upon HCT, suggesting it reflects tissue penetration, therefore, measuring enzyme activity in these body fluids could be helpful in the evaluation of the effect of therapy in tissues secluded from the circulation. Yearly enzyme activity levels for the first 1-3 years post-transplantation is appropriate. Since it appears to stabilize after HCT, thereafter, less frequently is reasonable. The residual substrate level, can be assessed by measuring the urinary GAG excretion.

Historically, residual substrate level analysis is performed by quantification of urinary GAGs by a dimethylmethylene blue dye binding assay (DMB), followed by two-dimensional electrophoresis for qualification of the type of excreted GAGs. The DMD assay, however, is very time consuming and may lead to false-negative results, especially in MPS IV as it failed to detect KS. Therefore, a more sensitive method was developed by liquid chromatography tandem-mass spectrometry (LC-MS/MS) which can detect HS, DS, and KS with high sensitivity. ${ }^{13}$ The possibility to detect KS levels is especially of interest as plasma levels of KS have been reported to correlate with skeletal dysplasia in MPS patients and KS levels could thus be a potential biomarker for the effect of treatment on skeletal disease. ${ }^{14}$

Hepatosplenomegaly, present in most untreated MPS patients, is rapidly reduced following HCT. ${ }^{5,15,16}$ After normalization, evaluation of hepatosplenomegaly is no longer required. Another characteristic manifestation in generally all MPS patients is severe and progressive growth retardation, accompanied by growth hormone deficiency in some of the patients. ${ }^{17}$ Although linear growth is maintained post-HCT for several years, a significantly reduced longitudinal height is seen in long-term survivors. ${ }^{5,8,15}$ Yearly evaluation of the growth is therefore recommended. Furthermore, the metabolic pediatrician should be aware of potential HCT-related endocrinologic complications, including growth hormone deficiency, adrenal insufficiency, thyroid dysfunction and gonadal dysfunction, especially in long-term survivors, which will also be addressed later.

\section{Neurodevelopment}

Without HCT, MPS patients with CNS involvement (MPSIH, MPSIIA, MPSIII) will inevitably show progressive neurocognitive deterioration. The degree of deterioration highly depends on the severity of the underlying MPS disorder and its severity. For instance, attenuated MPSI patients may experience little to no neurologic or developmental deterioration, while it is universally seen in the severe phenotype of MPSI, Hurler syndrome. After HCT, many 
MPS type I and II patients continued to gain skills, although often at a slightly slower rate as compared to unaffected children. ${ }^{5,6,8,15,18}$ As enzyme therapy is available for MPSII, it is assumed that the primary reason to consider transplantation is to prevent neurologic decline. However, the phenotype is not completely predicted by the genotype. In addition, waiting for clear neurocognitive decline prior to transplant will result in neurologic disease that will not be reversible. This results in difficulty in establishing which MPSII patients may best benefit from transplantation. The cognitive outcomes of patients with MPSIH post-transplant depends on the age at which transplant is performed, the preparative regimen used, the baseline functioning before transplant and the sex of the patient. ${ }^{8}$ This information was an important factor in the rationale to perform newborn screening for MPSI. The neurocognitive outcome in MPS type III patients is less favorable, with severe developmental delay despite HCT. ${ }^{6,19-}$

${ }^{21}$ It cannot be excluded that the deteriorating neurodevelopmental course in MPS type III patients could be more effectively treated if successful HCT is performed in asymptomatic patients. However, at this moment, experimental gene therapies, such as autologous genemodified hematopoietic cell transplantation or gene therapies directly targeting the brain, most likely have the best potential for better treatment outcomes in MPS type III patients. Often, accompanying motor difficulties as well as residual vision and hearing impairment contribute to the neurocognitive impairment.

It is recommended to evaluate the neurocognitive status in transplanted MPS patients with CNS involvement at a regular interval, preferably yearly during the first three years after HCT. Thereafter, establishing the limitations of individuals will prove important with regards to determining what services might optimize functioning (speech pathology, for instance), and what the best approaches might be used in an educational setting and at home. For prediction of the long-term neurocognitive prognosis, a baseline neurocognitive evaluation pre-HCT is essential, as it predicts post-HCT cognition. ${ }^{8}$ However, this evaluation must be interpreted with caution, also since reversible hearing or visual impairment can severely impact the neurocognitive functioning of MPS patients at time of HCT.

\section{Neurology}

One of the most severe neurologic manifestations observed in MPS type I, II, and III patients is a progressive communicating hydrocephalus, contributing to increased intracranial pressure that may lead to neurocognitive dysfunction in untreated patients. After HCT, hydrocephalus is typically either prevented or stabilized and ventriculo-peritoneal shunting is rarely required. ${ }^{5,7,8,18}$ This is accompanied by a normalizing head circumference. ${ }^{5}$ Progressive cerebral atrophy and accumulation of GAGs in the Virchow-Robin spaces, observed in untreated MPS patients with CNS involvement, is arrested after HCT. ${ }^{5,7,8,18,22}$ Screening for hydrocephalus by neurologic evaluation and cerebral magnetic resonance imaging (MRI) is 
essential pre-transplant and in the first three years post-transplant in all MPS type I, II, and III patients. The frequency of subsequent evaluation of the cerebral status can be based on clinical judgment.

Spinal cord compression, which may occur at the cervical or thoracolumbar region, is rarely seen early after transplantation with full donor-derived chimerism. ${ }^{8,18}$ However, the stenosis of the spinal canal at the cervical or thoracolumbar region is still present. As the thoracolumbar kyphosis is progressive in $70-80 \%$ of the children ${ }^{23}$, it is not excluded that spinal cord compression might occur at a later stage in transplanted patients. Patients that are transplanted at an older age, or with suboptimal enzyme levels post-HCT are at a substantial risk. The severity of this complication warrants frequent monitoring by performing a MRI of the spine in all patients. We suggest this to be evaluated starting from the age of 5 years, and every 2 to 3 years thereafter unless a heightened concern based on prior scans is present. Severe kyphosis or neurological deterioration should prompt additional imaging.

Carpal tunnel syndrome (CTS) is a common finding in various MPS types, including types I, II and VI, and is frequently observed despite HCT. ${ }^{8,15,17,24,25}$ The development of CTS is multi-factorial, with changes to the anatomy of the carpal tunnel and accumulation of storage material along the tendons contributing to compression of the median nerve. It is important to realize that the characteristic symptoms of carpal tunnel syndrome are rarely reported by MPS patients, and can be masked by other features of the disease. ${ }^{17}$ Nerve conduction studies (NCV), electromyography (EMG) and/or ultrasound are therefore required every 1-2 years post transplantation. If CTS is present, surgical release should be performed to prevent muscle wasting and irreversible damage to the median nerve. ${ }^{26}$ Post-release evaluation with NCV, EMG or ultrasound is recommended, as a high recurrence rate is observed. Unfortunately, NCV and EMG studies do not always normalize after release. Therefore, there is a need for better diagnostic modalities. Furthermore, the high occurrence rate gives reason to question whether surgical release is the best treatment for CTS in MPS patients. However, as the exact mechanism behind CTS in MPS patients is still an unresolved issue, surgical release remains the standard treatment. "Trigger digits" may also be observed, and require intervention. Evaluation of a hand specialist in addition to the assessment of nerve function is important.

\section{Orthopedics}

There is a characteristic pattern of skeletal deformities observed in all MPS types, including thoracolumbar kyphosis, acetabular dysplasia, and genu valgum, especially in MPSI and MPSVI. ${ }^{5}$ Despite HCT, these skeletal manifestations still progress in the majority of the patients, although apparently with later onset and a slower rate of progression. ${ }^{15,24,27}$ Surgical intervention is often required. The residual skeletal manifestations are at least in part 
responsible for the functional limitations and affected longitudinal height of these patients post-HCT. ${ }^{8}$ Quality of life and ability to engage in activities is affected. ${ }^{28}$ A lower age at HCT and normal white blood cell enzyme activity levels after HCT appeared to be significant predictors for superior orthopedic outcome post-HCT in MPS I-Hurler syndrome patients. ${ }^{8}$

Consensus guidelines have been developed regarding follow up and treatment of skeletal problems in MPSI patients, especially for the hip ${ }^{29}$ and the back. ${ }^{30}$ The skeletal abnormalities (total spine, hips and knees) should be visualized yearly using radiological imaging and evaluated by an orthopedic surgeon experienced in MPS diseases. It can be very useful to consult a physical or occupational therapist for assessment of the functional limitations, and strategies to maximize range of motion. Since patients with MPS type III have relative mild orthopedic involvement, the frequency of orthopedic evaluation in these patients should be based on clinical judgment.

\section{Cardiology}

Prior to the use of transplantation for MPSI-Hurler, most patients died of cardio-pulmonary disease prior to the age of 10 . Death resulting from cardiomyopathy, coronary artery occlusion or valvular dysfunction has become very uncommon in MPS patients achieving successful engraftment. ${ }^{5,8}$ In contrast to the significant beneficial effects on the myocardium, progression of mitral and aortic valve insufficiency is still seen in a significant proportion of the transplanted patients. Fortunately, the observed valve insufficiencies rarely warrant valve replacement, although treatment with an ACE-inhibitor can be required to prevent deterioration of cardiac function. ${ }^{5-8,24}$ Therefore, yearly follow-up by electrocardiogram (ECG) and cardiac ultrasound is of high importance in MPS patients. Furthermore, an atrioventricular block, which is a very rare but potentially life-threatening finding in longterm survivors, ${ }^{5}$ should be considered and excluded by (Holter-)ECG on individual basis, especially before receiving extensive surgical intervention. It should be recognized that the first transplant for an MPS disorder took place less than 40 years ago, and so the long-term cardiac outcomes of these patients is not yet fully established.

\section{Ear, nose, and throat}

Shortly after HCT, the persistent rhinorrhea and recurrent upper airway infections and obstruction improve in the majority of the MPS patients. ${ }^{5,15}$ Recurrent upper respiratory infections do occur despite HCT however, frequently requiring ear, nose, and throat (ENT) surgery. Obstructive sleep apnea is markedly decreased and only rarely requires respiratory support, although patients with mixed chimerism or low post-transplant enzyme activity levels may be more affected. ${ }^{8,24,31,32}$ Polysomnography to screen for obstructive sleep apnea should be considered based on clinical judgment. Signs of obstructive airway problems 
(snoring, sleep apnea, daytime sleepiness, morning headaches) would prompt further sleep studies, e.g. polysomnography.

Hearing loss - a universal problem in untransplanted patients - is a frequent finding during follow up, requiring hearing aids in a significant part of the transplanted MPS patients. ${ }^{8,15,24,33,34}$ The hearing loss can be of conductive, sensorineural, or mixed type. The first years posttransplant, the majority of patients exhibit conductive hearing loss which improves over time. Surgical intervention such as ventilation tubes are necessary in a significant number of patients. Sensorineural hearing loss however, increases slowly over time and could eventually lead to the need of a cochlear implant. ${ }^{35}$ Yearly follow-up by an ENT specialist is therefore recommended, at least in patients with MPS type I, II, and VI, including the evaluation of the hearing status.

The exact mechanism of the sensorineural hearing loss is not yet unraveled. However, post-mortem analysis of the inner ear of MPS I patients showed a reduced number of inner and outer hair cells in the cochlea. ${ }^{36}$ In the future, otoacoustic emissions and advanced electrophysiological measurements might reveal more insight in the pathophysiology of sensorineural hearing loss.

\section{Ophthalmology}

Glaucoma, either open-, or narrow-angled is reported in MPS types I and VI. ${ }^{37}$ Owing to the stiffness of the cornea as a result of GAG accumulation, it can be difficult to adequately measure the intra-ocular pressure in these patients also post-transplant. ${ }^{12,38}$ Of note, glaucoma surgery is rarely necessary in transplanted patients. Optic disc swelling and subsequent nerve atrophy, which may occur in untreated MPS patients due to (a combination of) increased intracranial pressure, increased ocular pressure, or GAG-mediated optic nerve compression, is rarely observed after successful HCT. ${ }^{5}$

The characteristic corneal clouding, which will lead to severe visual impairment without HCT in all except MPS type II patients, is progressive in transplanted patients. ${ }^{12,37,39,40}$ In general, corneal transplantation is not recommended in children because of high rejection rates. ${ }^{41}$ Furthermore, retinal degeneration, opticopathy, or amblyopia, also hampering visual acuity, can be present despite HCT and are not resolved with a corneal transplant. Therefore, patients with greatly reduced visual acuity should receive additional testing, including visual field, optical coherence tomography, and electroretinography. When these are normal, corneal transplantation could be considered in older patients. ${ }^{42}$ In case of night blindness, electroretinography should also be performed to determine the presence of retinal degeneration. There is increasing appreciation for the presence of retinal degeneration in MPS patients. The exact pathophysiology, however, is unknown. Future studies, with detailed evaluation of the retina in MPS patients would be helpful in acquiring more knowledge 
concerning this question. Due to the various ocular features in MPS patients, the majority of the patients require glasses, also in the transplanted population. Yearly ophthalmologic monitoring including the evaluation of corneal clouding, visual acuity, refractive error, retina, and intra-ocular pressure is recommended. ${ }^{43}$

\section{Transplant-related morbidity}

Besides residual disease, transplanted related morbidity could also be seen during the followup of these patients, including graft-versus-host disease in the early years post-HCT, and endocrine problems in long-term follow-up. Assessment of these morbidities is important as intervention could be necessary. Especially the first 1-2 years post-HCT, symptoms of chronic graft-versus-host-disease (GVHD) could still be encountered. Chronic GVHD occurs usually in the skin and nails, joints and muscles, eyes, mouth, lungs, digestive system or genitals. ${ }^{44}$ Growth retardation remains an important problem after HCT in MPS patients, mostly due to skeletal disease. Endocrine problems, however, that further compound the already impaired growth potential should be evaluated, including growth hormone deficiency and hypothyroidism. Secondary adrenal insufficiency may be seen in patients after longterm steroid treatment, and should be considered in stress-situations or operations. Gonadal dysfunction is very frequent, and especially in females may lead to delayed or arrested puberty, requiring hormone supplementation for pubertal induction. ${ }^{45}$ Historically, conditioning regimens have included total body irradiation, which is associated with specific long term morbidity including cataract development. ${ }^{5}$

\section{CONCLUSIONS}

Residual disease burden is observed in MPS patients despite HCT. Systematic follow-up of these patients is required by providers familiar with these disorders in order to anticipate and recognize the various aspects of this residual disease at an early stage, allowing for timely intervention if necessary. Also, this information can be of great use to the parents and other providers of these affected children in order to prevent complications and maximize quality of life. Ideally, all MPS patients should be evaluated at baseline and yearly post-transplant by a multidisciplinary team experienced in MPS disease. As the follow-up age increases, this frequency might be reduced based on clinical judgment of the involved subspecialists. The vast majority of this care is now delivered by pediatric providers, but it is clear that as this population increases in age, identifying dedicated providers of adult care will prove necessary. Furthermore, as these are rare disorders, mechanisms of pooling data as to outcomes across institutions would be of great importance in expanding our knowledge of disease related complications. 
Table 2. Baseline and follow-up evaluation after HCT

\begin{tabular}{|c|c|c|c|c|c|}
\hline Subspecialty & Diagnostic procedure & Manifestation & Baseline & $\begin{array}{c}\text { Follow-up } \\
1-3 \text { years } \\
\text { post-HCT } \\
\end{array}$ & $\begin{array}{l}\text { Follow-up } \\
\text { from } 4 \text { years } \\
\text { post-HCT }\end{array}$ \\
\hline \multirow[t]{4}{*}{ Metabolic diseases } & \multirow[t]{2}{*}{$\begin{array}{l}\text { Biochemical } \\
\text { evaluation: laboratory }\end{array}$} & $\begin{array}{l}\text { Lysosomal enzyme activity } \\
\text { in blood and saliva }\end{array}$ & Yes & Yearly & N.A. \\
\hline & & $\begin{array}{l}\text { Urine: storage product } \\
\text { excretion }\end{array}$ & Yes & Yearly & Yearly \\
\hline & \multirow[t]{2}{*}{$\begin{array}{l}\text { General paediatric } \\
\text { evaluation }\end{array}$} & Hepatosplenomegaly & Yes & $\begin{array}{c}\text { Until } \\
\text { normal }\end{array}$ & N.A. \\
\hline & & Growth retardation & Yes & Yearly & $\begin{array}{c}\text { Every } 2 \\
\text { years }\end{array}$ \\
\hline Neurodevelopment & $\begin{array}{l}\text { Neurodevelopmental } \\
\text { test }\end{array}$ & Neurodevelopment & Yes & Yearly, e & $\begin{array}{l}\text { Every } 2 \\
\text { years }^{\mathrm{d}, \mathrm{e}}\end{array}$ \\
\hline \multirow[t]{4}{*}{ Neurology } & $\begin{array}{l}\text { General neurologic } \\
\text { evaluation }\end{array}$ & $\begin{array}{l}\text { Hydrocephalus, } \\
\text { myelopathy, neuropathy }\end{array}$ & Yes & Yearly $^{\mathrm{d}, \mathrm{e}}$ & $\begin{array}{l}\text { Every } 2 \\
\text { years }^{\mathrm{d}, \mathrm{e}}\end{array}$ \\
\hline & Cerebral MRI & $\begin{array}{l}\text { Hydrocephalus, } \\
\text { Cerebral atrophy, } \\
\text { White matter lesions }\end{array}$ & Yes & Yearly & N.A. \\
\hline & $\begin{array}{l}\text { Spinal MRI/ } \\
\text { Neurophysiologic test }\end{array}$ & Cord compression & Yes & $\begin{array}{c}\text { Every } 2 \\
\text { years }^{\mathrm{c}}\end{array}$ & $\begin{array}{c}\text { Every } 2 \\
\text { years }^{\mathrm{c}}\end{array}$ \\
\hline & $\mathrm{EMG} / \mathrm{NCV}$ & Carpal tunnel syndrome & Yes & Yearly $^{\mathrm{c}, \mathrm{d}}$ & Yearly ${ }^{\mathrm{c}, \mathrm{d}}$ \\
\hline Orthopedics & $\begin{array}{l}\text { General orthopedic } \\
\text { evaluation } \\
\text { X-ray total spine, hips } \\
\text { \& knees }\end{array}$ & $\begin{array}{l}\text { Joint stiffness, } \\
\text { Thoracolumbar kyphosis, } \\
\text { Hip dysplasia, } \\
\text { Genu valgum }\end{array}$ & Yes & Yearly $^{\mathrm{c}}$ & Yearly $^{\mathrm{c}}$ \\
\hline \multirow[t]{2}{*}{ Cardiology } & $\begin{array}{l}\text { ECG \& cardiac } \\
\text { ultrasound }\end{array}$ & $\begin{array}{l}\text { Cardiac function, } \\
\text { Valve insufficiency }\end{array}$ & Yes & Yearly $^{\mathrm{c}, \mathrm{d}}$ & $\begin{array}{c}\text { Every } 2 \\
\text { years }\end{array}$ \\
\hline & (Holter-)ECG & Arrhythmias & $* \mathrm{a}$ & $* \mathrm{a}$ & $* \mathrm{a}$ \\
\hline \multirow[t]{2}{*}{ ENT } & $\begin{array}{l}\text { General ENT } \\
\text { evaluation } \\
\text { Hearing test (tone } \\
\text { audiometry/BAEP) }\end{array}$ & $\begin{array}{l}\text { Upper airway, Infection/ } \\
\text { obstruction, } \\
\text { Hearing loss }\end{array}$ & Yes & Yearly ${ }^{\mathrm{c}, \mathrm{d}}$ & Yearly $^{\mathrm{c}, \mathrm{d}}$ \\
\hline & Polysomnography & Sleep apnea & $* \mathrm{a}$ & $* \mathrm{a}$ & $* \mathrm{a}$ \\
\hline \multirow[t]{2}{*}{ Ophthalmology } & $\begin{array}{l}\text { Vision test, retina \& } \\
\text { IOP test }\end{array}$ & $\begin{array}{l}\text { Visual acuity, } \\
\text { Corneal clouding, } \\
\text { Optic nerve atrophy, } \\
\text { Glaucoma }\end{array}$ & Yes & Yearly $^{\mathrm{b}}$ & Yearly $^{\mathrm{b}}$ \\
\hline & ERG & Retinopathy & $* \mathrm{a}$ & $* \mathrm{a}$ & $* \mathrm{a}$ \\
\hline
\end{tabular}




\section{REFERENCES}

1. Muenzer J. Overview of the mucopolysaccharidoses. Rheumatology. 2011; 50: 4-12.

2. Hobbs JR, Hugh-Jones K, Barrett AJ, et al. Reversal of clinical features of Hurler's disease and biochemical improvement after treatment by bone-marrow transplantation. Lancet (London, England). 1981; 2: 709-712.

3. Boelens JJ, Aldenhoven M, Purtill D, et al. Outcomes of transplantation using various hematopoietic cell sources in children with Hurler syndrome after myeloablative conditioning. Blood. 2013;121.

4. Taylor M, Khan S, Stapleton M, et al. Hematopoietic stem cell transplantation for mucopolysaccharidoses; past, present, and future. Biol Blood Marrow Transplant. 2019.

5. Aldenhoven M, Boelens JJ, de Koning TJ. The clinical outcome of Hurler syndrome after stem cell transplantation. Biol Blood Marrow Transplant. 2008; 14: 485-498.

6. Prasad VK, Mendizabal A, Parikh SH, et al. Unrelated donor umbilical cord blood transplantation for inherited metabolic disorders in 159 pediatric patients from a single center: influence of cellular composition of the graft on transplantation outcomes. Blood. 2008; 112: 2979-2989.

7. Tanaka A, Okuyama T, Suzuki Y, et al. Long-term efficacy of hematopoietic stem cell transplantation on brain involvement in patients with mucopolysaccharidosis type II: a nationwide survey in Japan. Mol Genet Metab. 2012; 107: 513-520.

8. Aldenhoven M, Wynn RF, Orchard PJ, et al. Long-term outcome of Hurler syndrome patients after hematopoietic cell transplantation: an international multicenter study. Blood. 2015; 125: 2164-2172.

9. Wadhwa A, Chen Y, Holmqvist A, et al. Late mortality after allogeneic blood or marrow transplantation for inborn errors of metabolism: a report from the blood or marrow transplant survivor Study-2 (BMTSS-2). Biol Blood Marrow Transplant. 2019; 25: 328-334.

10. Tan EY, Boelens JJ, Jones SA, Wynn RF. Haematopoietic Stem Cell Transplantation in Inborn Errors of Metabolism-On Behalf of IEWP-EBMT. Front Pediatr. 2019;7:433.

11. Doorn J van, van den Broek BTA, Geboers AJ, Kuiper G-A, Boelens JJ, van Hasselt PM. Salivary $\alpha$-Iduronidase Activity as a Potential New Biomarker for the Diagnosis and Monitoring the Effect of Therapy in Mucopolysaccharidosis Type I. Biol Blood Marrow Transplant. 2018:1-6.

12. van den Broek BTA, van Egmond-Ebbeling MB, Achterberg JA, et al. Longitudinal analysis of ocular disease in children with Mucopolysaccharidosis I after 
hematopoietic cell transplantation. Biol Blood Marrow Transplant. November 2019.

13. Langereis EJ, Wagemans T, Kulik W, et al. A Multiplex Assay for the Diagnosis of Mucopolysaccharidoses and Mucolipidoses. Karamanos NK, ed. PLoS One. $2015 ; 10$.

14. Rowan DJ, Tomatsu S, Grubb JH, Montaño AM, Sly WS. Assessment of bone dysplasia by micro-CT and glycosaminoglycan levels in mouse models for mucopolysaccharidosis type I, IIIA, IVA, and VII. J Inherit Metab Dis. 2013; 36: 235-246.

15. Guffon N, Bertrand Y, Forest I, Fouilhoux A, Froissart R. Bone marrow transplantation in children with Hunter syndrome: outcome after 7 to 17 years. J Pediatr. 2009; 154: 733-737.

16. Jester S, Larsson J, Eklund EA, et al. Haploidentical stem cell transplantation in two children with mucopolysaccharidosis VI: clinical and biochemical outcome. Orphanet J Rare Dis. 2013; 8: 134.

17. Aldenhoven M, Sakkers RJB, Boelens J, de Koning TJ, Wulffraat NM. Musculoskeletal manifestations of lysosomal storage disorders. Ann Rheum Dis. 2009; 68: 1659-1665.

18. Vellodi A, Young E, Cooper A, Lidchi V, Winchester B, Wraith JE. Long-term follow-up following bone marrow transplantation for Hunter disease. $J$ Inherit Metab Dis. 1999; 22: 638-648.

19. Sivakumur P, Wraith JE. Bone marrow transplantation in mucopolysaccharidosis type IIIA: a comparison of an early treated patient with his untreated sibling. $J$ Inherit Metab Dis. 1999; 22:849.

20. VellodiA, Young E, New M, Pot-Mees C, Hugh-Jones K. Bone marrow transplantation for Sanfilippo disease type B. J Inherit Metab Dis. 1992; 15: 911-918.

21. Welling L, Marchal JP, van Hasselt P, van der Ploeg AT, Wijburg FA, Boelens JJ. Early Umbilical Cord Blood-Derived Stem Cell Transplantation Does Not Prevent Neurological Deterioration in Mucopolysaccharidosis Type III. JIMD Rep. 2014; 18: 63-68.

22. Annibali R, Caponi L, Morganti A, Manna M, Gabrielli O, Ficcadenti A. Hunter syndrome (Mucopolysaccharidosis type II), severe phenotype: long term follow-up on patients undergone to hematopoietic stem cell transplantation. Minerva Pediatr. 2013; 65: 487-496.

23. van der Linden MH, Kruyt MC, Sakkers RJB, de Koning TJ, Oner FC, Castelein RM. Orthopaedic management of Hurler's disease after hematopoietic stem cell transplantation: a systematic review. J Inherit Metab Dis. 2011; 34: 657-669.

24. Herskhovitz E, Young E, Rainer J, et al. Bone marrow transplantation for Maroteaux- 
Lamy syndrome (MPS VI): long-term follow-up. J Inherit Metab Dis. 1999; 22:5062.

25. Khanna G, Van Heest AE, Agel J, et al. Analysis of factors affecting development of carpal tunnel syndrome in patients with Hurler syndrome after hematopoietic cell transplantation. Bone Marrow Transplant. 2007; 39: 331-334.

26. Van Heest AE, House J, Krivit W, Walker K. Surgical treatment of carpal tunnel syndrome and trigger digits in children with mucopolysaccharide storage disorders. J Hand Surg Am. 1998; 23: 236-243.

27. Langereis EJ, den Os MM, Breen C, et al. Progression of Hip Dysplasia in Mucopolysaccharidosis Type I Hurler After Successful Hematopoietic Stem Cell Transplantation. J Bone Joint Surg Am. 2016; 98: 386-395.

28. Aldenhoven M, A van den BBT, Wynn RF, et al. Quality of life of Hurler syndrome patients after successful hematopoietic stem cell transplantation. Blood Adv. Nov 2017.

29. Langereis EJ, Borgo A, Crushell E, et al. Treatment of hip dysplasia in patients with mucopolysaccharidosis type I after hematopoietic stem cell transplantation: results of an international consensus procedure. Orphanet J Rare Dis. 2013; 8: 155.

30. Kuiper G-A, Langereis EJ, Breyer S, et al. Treatment of thoracolumbar kyphosis in patients with mucopolysaccharidosis type I: results of an international consensus procedure. Orphanet J Rare Dis. 2019; 14: 17.

31. Sillence D, Waters K, Donaldson S, Shaw PJ, Ellaway C. Combined enzyme replacement therapy and hematopoietic stem cell transplantation in mucopolysacharidosis type VI. JIMD Reports-Case Res Reports. 2011

32. Pal AR, Langereis EJ, Saif MA, et al. Sleep disordered breathing in mucopolysaccharidosis I: a multivariate analysis of patient, therapeutic and metabolic correlators modifying long term clinical outcome. Orphanet J Rare Dis. 2015; 10: 42.

33. Papsin BC, Vellodi A, Bailey CM, Ratcliffe PC, Leighton SE. Otologic and laryngologic manifestations of mucopolysaccharidoses after bone marrow transplantation. Otolaryngol Head Neck Surg. 1998; 118: 30-36.

34. Da Costa V, O’Grady G, Jackson L, Kaylie D, Raynor E. Improvements in sensorineural hearing loss after cord blood transplant in patients with mucopolysaccharidosis. Arch Otolaryngol Head Neck Surg. 2012; 138: 1071-1076.

35. van den Broek BTA, Smit AL, Boelens JJ, van Hasselt PM. Hearing loss in patients with Mucopolysaccharidoses after hematopoietic cell transplantation; a longitudinal analysis. Submitted.

36. Kariya S, Schachern PA, Nishizaki K, Paparella MM, Cureoglu S. Inner ear changes 
in mucopolysaccharidosis type I/Hurler syndrome. Otol Neurotol Off Publ Am Otol Soc Am Neurotol Soc [and] Eur Acad Otol Neurotol. 2012; 33: 1323-1327.

37. Summers CG, Fahnehjelm KT, Pitz S, et al. Systemic therapies for mucopolysaccharidosis: Ocular changes following haematopoietic stem cell transplantation or enzyme replacement therapy - a review. Clin Exp Ophthalmol. 2010; 38: 34-42.

38. Fahnehjelm KT, Chen E, Winiarski J. Corneal hysteresis in mucopolysaccharidosis I and VI. Acta Ophthalmol. 2012; 90: 445-448.

39. Gullingsrud EO, Krivit W, Summers CG. Ocular abnormalities in the mucopolysaccharidoses after bone marrow transplantation. Longer follow-up. Ophthalmology. 1998; 105: 1099-1105.

40. Javed A, Aslam T, Jones SA, et al. The effect of haemopoietic stem cell transplantation on the ocular phenotype in mucopolysaccharidosis type I (Hurler). Acta Ophthalmol. 2017: $1-5$.

41. Limaiem R, Chebil A, Baba A, Ben Youssef N, Mghaieth F, El Matri L. Pediatric Penetrating Keratoplasty: Indications and Outcomes. Transplant Proc. 2011; 43: 649-651.

42. Ohden KL, Pitz S, Ashworth J, et al. Outcomes of keratoplasty in the mucopolysaccharidoses: an international perspective. Br J Ophthalmol. 2017; 101: 909-912.

43. Fahnehjelm KT, Ashworth JL, Pitz S, et al. Clinical guidelines for diagnosing and managing ocular manifestations in children with mucopolysaccharidosis. Acta Ophthalmol. 2012; 90: 595-602.

44. Penack O, Marchetti M, Ruutu T, et al. Prophylaxis and management of graft versus host disease after stem-cell transplantation for haematological malignancies: updated consensus recommendations of the European Society for Blood and Marrow Transplantation. Lancet Haematol. 2020; 7: 157-167.

45. Shalitin S, Pertman L, Yackobovitch-Gavan M, et al. Endocrine and Metabolic Disturbances in Survivors of Hematopoietic Stem Cell Transplantation in Childhood and Adolescence. Horm Res Paediatr. 2018; 89: 108-121. 

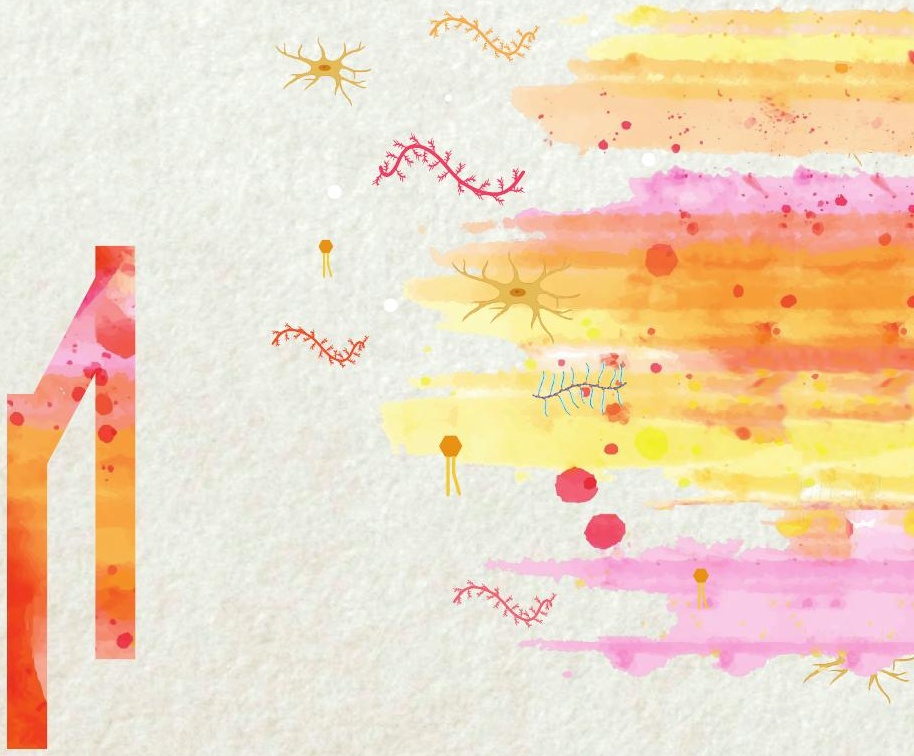


\section{PART II}

Pathophysiology of Disease

Progression after Hematopoietic

Cell Transplantation 


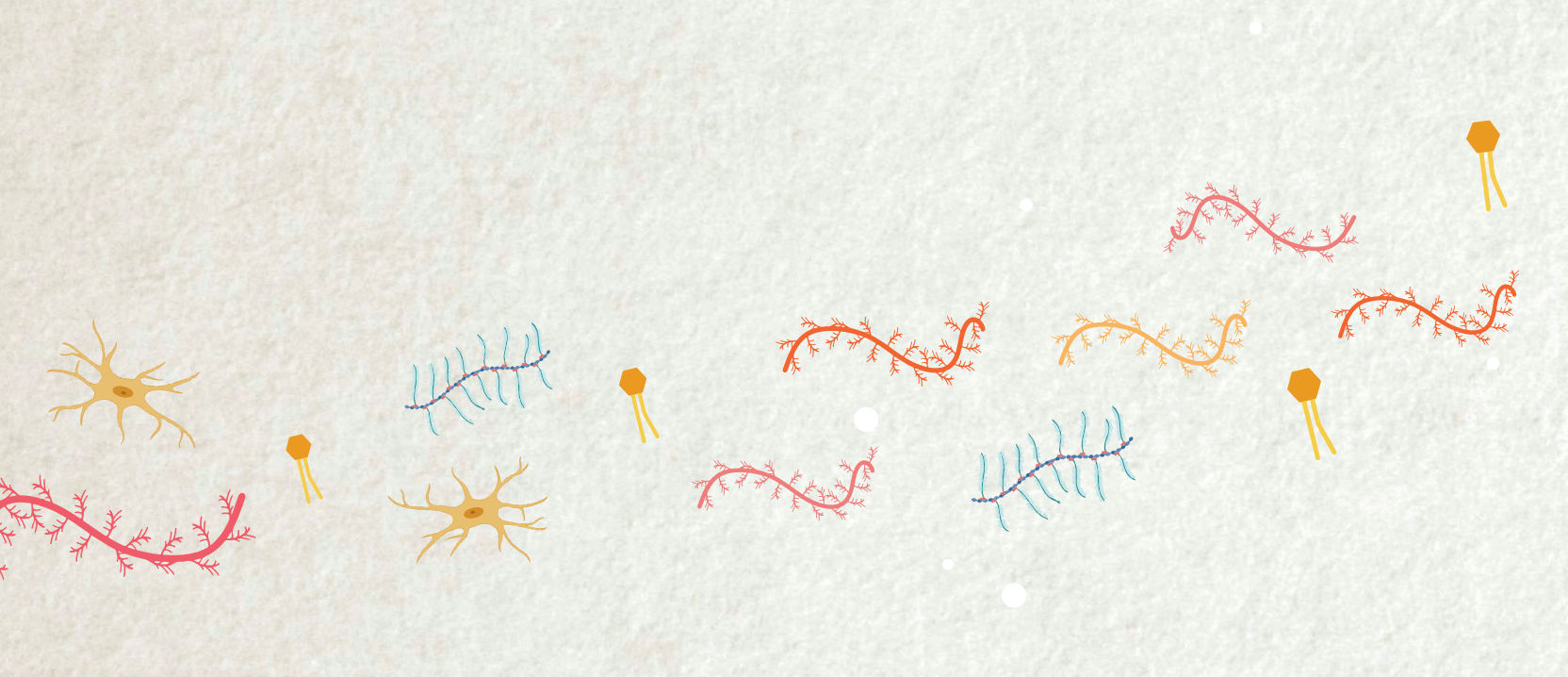



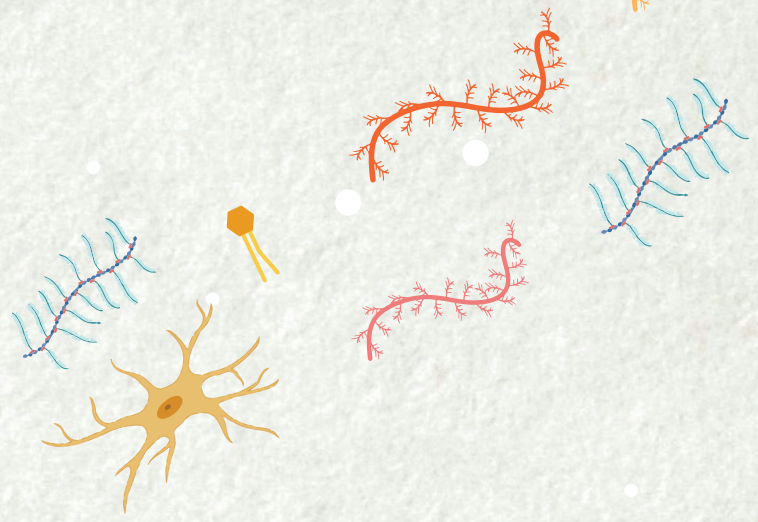

\section{CHAPTER 7}

\section{Salivary $\alpha$-Iduronidase Activity as a Potential New Biomarker for Diagnosing and Monitoring the Effect of Therapy in Mucopolysaccharidosis I}

Brigitte T.A. van den Broek* Jaap van Doorn*

Ans J. Geboers

Ge-Ann Kuiper

Jaap Jan Boelens

Peter M. van Hasselt

*Authors contributed equally 


\begin{abstract}
Although disease progression in Mucopolysaccharidosis I (MPS-1) can be attenuated by hematopoietic cell transplantation (HCT), it is increasingly recognized that residual disease is substantial. Biomarkers that would allow us to evaluate the efficacy of HCT (and upcoming new therapies) in non-hematological tissues are needed. Current biomarkers, including the iduronidase (IDUA) activity in leukocytes, are not suitable for this purpose as they are assessed in tissues of hematological origin and may not reflect enzyme availability in non-hematologic tissues. Saliva is a non-hematological body fluid which can be collected easily and noninvasively. We hypothesized that the extent of recovery of IDUA activity in saliva after HCT could provide better understanding of the penetration of donor derived enzyme into nonhematological compartments. This study in 20 MPS-1 patients shows that the measurement of IDUA activity in saliva is possible and allows diagnosis of IDUA deficiency $(p<0.0001)$, with values a magnitude further deviating from the normal range than when assayed in corresponding dried blood spots (DBS). Furthermore, it could possibly differentiate between phenotypes ( $p=0.045$ ). More importantly, patients exhibit strikingly low values of IDUA in saliva after HCT, far below the normal range of controls $(\mathrm{p}=0.013)$, contrasting the normal IDUA levels in DBS. We postulate that the limited recovery of donor-derived IDUA activity in saliva after treatment reflects the situation in poorly responding non-hematological tissue compartments unveiling enzyme delivery as a weak spot of the current therapy. Salivary IDUA activity could be used as a biomarker for the evaluation of the effect of new therapies in well vascularized non-hematological tissues.
\end{abstract}




\section{INTRODUCTION}

Mucopolysaccharidosis type I (MPS-1) is an autosomal recessive inherited deficiency of the enzyme $\alpha$-iduronidase (IDUA; EC 3.2.1.76), which leads to accumulation of glycosaminoglycans within the lysosomes. MPS-1 includes a spectrum of clinical phenotypes ranging from mild to severe. Hurler syndrome (MPS-1H) represents the most severe subtype, resulting in progressive and ultimately fatal multisystem disease. ${ }^{1,2}$ At present, haematopoietic cell transplantation (HCT), based on the principle of cross-correction, is the only treatment able to prevent deterioration of the central nervous system. ${ }^{3,4}$ Enzyme replacement therapy (ERT), which is applied in milder variants (i.e. Hurler/Scheie and Scheie), is limited to the peripheral nervous system as it cannot pass the blood-brain-barrier and furthermore stimulates the production of neutralizing antibodies. ${ }^{5}$ An increased life expectancy and improvement of clinical outcome parameters have been reported after HCT, although patients with enzyme levels below the lower limit of normal show worse outcomes. ${ }^{6}$ This has resulted in treatment regimens striving for high enzyme levels in the majority of the patients. However, despite normal levels of IDUA activity in either DBS, plasma or leukocytes, residual disease burden is still present in all patients with MPS-1H. ${ }^{6}$ Insufficient tissue penetration is likely an important factor but biomarkers to monitor and to predict the biochemical effects of treatment in nonhematological tissues are lacking.

Saliva may be an interesting tool representing tissue penetration. It is an easily accessible body fluid and of non-hematological origin as its content is derived from salivary glands and buccal epithelial cells. ${ }^{7,8}$ The oral cavity is well vascularized and is not enclosed by a barrier such as the blood-brain-barrier. Therefore, the extent of recovery of IDUA activity in saliva could provide more insight in the transport to non-hematological compartments. The literature on salivary lysosomal hydrolases is scarce, although it has been shown that the activities of e.g. hexoaminidase A and B, $\alpha$ - and $\beta$-glucosidase, and $\alpha$-galactosidase A can be readily assessed in human saliva. ${ }^{9-11}$ To our knowledge, no data are available for IDUA.

In this study, we investigated whether the measurement of IDUA activity in saliva is useful as an alternative for the detection of IDUA deficiency and subsequently used it to determine the fate of donor-derived salivary IDUA in the follow-up of MPS-1 patients who underwent HCT successfully. 


\section{PATIENTS AND METHODS}

\section{Patients}

Patients with Hurler or Hurler/Scheie either untreated, on ERT or after HCT were included in the study between December 2012 and January 2018. The parents of several patients were also included and were considered as obligate MPS-1 heterozygotes. Saliva was collected from subjects of all groups, dried blood spots from all group except the heterozygotes. Samples of blood and saliva of the patients on ERT were taken shortly (i.e. one or two days) before the next weekly administration of rhIDUA, when circulating IDUA levels are at a nadir. Of the MPS-1 patients after HCT, the blood samples for DBS were taken on the same day as the saliva samples. The activity of lysosomal $\alpha$-galactosidase A (AGAL) was determined simultaneously, as a reference for recovery and control. The results were compared with those obtained for DBS from the same individuals. DBS normative range values for IDUA $(\mathrm{n}=116$, age: $0.03-76$ years) and AGAL activities $(\mathrm{n}=176$, age: 0.01-77 years) had been obtained previously from selected populations of patients visiting the hospital (e.g. prior to minor surgery, screening/follow up for phenylalanine ketonuria), and healthy volunteers.

All procedures followed were in accordance with the ethical standards of the Helsinki Declaration of 1975, as revised in 2000. The collection of saliva and DBS have been performed in a university hospital setting and have been exempted for approval according to the responsible institutional committee Medical Research Involving Human Subjects Act. Part of this report is based on patient data acquired during care-as-usual. As far as applicable, informed consent was obtained from all subjects or their parents for being included in the study.

\section{Determination of IDUA and AGAL activities}

For Western blotting, samples of saliva were diluted in $5 \times$ Laemmli buffer containing $0.625 \mathrm{M}$ Tris- $\mathrm{HCl}$ pH 6.8, 250 mM DTT, 10\% SDS, 50\% glycerol, and 0.01\% Bromophenol blue. Samples were electrophoresed through 10\% SDS-PAA gels and blotted on Immobilon-FL PVDF (Millipore). Blots were analyzed for IDUA using sheep serum anti-hIDUA antibody (R\&D systems, Europe Ltd., Abingdon, UK). Secondary Alexa 680 fluorescent antibodies were used for detection in the Odyssey imaging system (Li-Cor). Immunoprecipitation of IDUA in saliva was performed by incubation of $100 \mu \mathrm{l}$ of 6 times diluted saliva samples with either 0.2 or $1.0 \mu \mathrm{g}$ sheep anti-hIDUA antibody for $1 \mathrm{~h}$ at room temperature, followed by incubation with $100 \mu 1$ anti-sheep/goat IgG-coated cellulose suspension (Sac-Cel, Immuno Diagnostic Systems, Boldon, UK). After 30 minutes, the suspension was centrifuged at $10.000 \mathrm{xg}$ for 10 minutes and the supernatant used for immediate determination of IDUA and AGAL specific activities. Complete protease inhibitor cocktail was obtained from Roche Diagnostics, Mannheim, Germany). 
Saliva was collected at least $1 \mathrm{~h}$ after the last meal or drink by the Sorbette system (Salimetrics, State College, PA, USA) at the same day as DBS collection. Samples were immediately stored at at $-80 \mathrm{oC}$, until analysis. DBS were prepared by dispensing whole blood or heparin plasma onto Guthrie cards. The measurements of IDUA (Wallac 1420-050 Multilabel Counter; Perkin Elmer, Turku, Finland) and AGAL (EC 3.2.1.22) activities in DBS and saliva were performed according to procedures described and validated previously by Chamoles et al., ${ }^{12,13}$ using 1.5 mm diameter disks (Wallac DBS puncher; Perkin Elmer, Turku, Finland) placed into 96 wells black micro Fluor plates (Greiner Bio-One B.V. Alphen aan de Rijn, the Netherlands). ${ }^{14}$ For both IDUA and AGAL, the results were calculated from the 4-methylumbelliferyl (MU) calibration curve and expressed as nmol per hour per ml for DBS or $\mathrm{mg}$ for saliva. For IDUA in DBS the intra- and inter assay variations are $11.3 \%(\mathrm{n}=24)$ and $11.5 \%(\mathrm{n}=34)$, at mean activities of 5.7 and $4.6 \mathrm{nmol} 4 \mathrm{MU} / \mathrm{h} . \mathrm{ml}$ blood, respectively. For AGAL the intra- and inter assay variations amounts 5\% $(n=21)$ and $11.3 \%(n=41)$, at mean activities of 3.6 and $5.3 \mathrm{nmol}$ 4MU/h.ml blood, respectively. The IDUA and AGAL intraassay variations ( $\mathrm{n}=16)$, as determined for 2 different control saliva samples, were $3.1 \%$ and $5.0 \%$, respectively. The IDUA and AGAL inter-assay variations for aliquots of saliva stored at $-80 \circ \mathrm{C}$ were $17.2 \%(n=11)$ and $14.8 \%(n=10)$, respectively. Protein contents of the various saliva samples were determined by the method of Lowry et al. ${ }^{15}$

\section{Statistics}

The limit of detection for $4 \mathrm{MU}$ production was calculated as $0.055 \mathrm{pmol} / \mathrm{well}$, corresponding to $\sim 0.06 \mathrm{nmol} / \mathrm{h} . \mathrm{ml}$ blood or $\mathrm{nmol} / \mathrm{h} . \mathrm{mg}$ saliva. To normalize data, log-transformation was performed. One-way ANOVA, Welch's T-test, or paired T-test was performed to calculate differences between (paired) groups. In case log-transformation was not sufficient according to the Shapiro-Wilk normality test, non-parametric tests (Kruskal-Wallis and, for paired samples, Wilcoxon signed rank test) were used.

\section{RESULTS}

\section{Patients}

Saliva and DBS samples were obtained from 4 untreated MPS-1H patients, 4 MPS- $1 \mathrm{H}$ patients on ERT while awaiting HCT, and 20 MPS-1H patients at least 6 months after HCT. Saliva was also collected from 13 heterozygotes. The median age at HCT was 1.25 yrs (range: 0.2$3.0 \mathrm{yrs}$ ). The median interval from HCT until sampling was 5.8 yrs (range: 0.5-12.3 yrs). In addition, saliva and DBS from 5 MPS-1H/S patients who only received ERT were analyzed. An overview of the various pathogenic mutations in IDUA are shown in table 1. 
Table 1. Overview of IDUA mutations in the various MPS-I patients included in the study.

\begin{tabular}{|c|c|}
\hline Subtype & Mutation \\
\hline $\begin{array}{l}\text { MPS-1H } \\
\geq 6 \text { months after HCT }(n=20)^{*}\end{array}$ & $\begin{array}{l}\text { Q70X/L218P; } \\
\text { A327P/A327P; } \\
\text { W402X/IVS1+1G>A; } \\
\text { W402X/L218P; W402X/ 134del12; } \\
\text { W402X/W402X; L218P/L218P; } \\
\text { A327P/R383H; W402X/Q70X; } \\
\text { A367E/ 1650del117; Q70X/Q70X; } \\
\text { c.1273dupC, p.His425fs /c.1273dupC; } \\
\text { c.1893delC, p.Phe632fs/ c.1893delC, p.Phe632fs; } \\
\text { Q70X, W402x; } \\
\text { c.1103_1104del,p.Arg368fs/c.1598C>G(p.Pro544Arg) }\end{array}$ \\
\hline $\begin{array}{l}\text { MPS-1H on ERT } \\
(n=4)\end{array}$ & $\begin{array}{l}\text { Q70X/L218P; A327P/A327P; } \\
\text { c.1273dupC, p.His425fs /c.1273dupC; } \\
\text { c.1893delC, p.Phe632fs/ c.1893delC, p.Phe632fs }\end{array}$ \\
\hline MPS-IH untreated $(n=4)$ & $\begin{array}{l}\text { Q70X, W402X*; } \\
\text { c.1103_1104del,p.Arg368fs/c.1598C>G(p.Pro544Arg); } \\
\text { c.653T>C,p. Leu218Pro; c. } 1205 \mathrm{G}>\text { A/p.Trp402 }\end{array}$ \\
\hline MPS-IH/S on ERT $(n=5)$ & $\begin{array}{ll}\text { W402X/Asn348Lys } & (n=2) \\
\text { P533R/P533R } & (n=2) \\
\text { L218P/D315Y } & \end{array}$ \\
\hline
\end{tabular}

\section{Measuring IDUA activity in saliva}

\section{Characterization of IDUA and AGAL activities}

Presence of IDUA enzyme was confirmed by Western blotting. Samples of a homogenate of cultured human fibroblasts showed a prominent band at $\sim 75 \mathrm{kD}$. In saliva, samples from two healthy individuals (with different levels of enzyme activities) showed minor immunoreactivity at an apparent molecular weight of about $\sim 75 \mathrm{kD}$, next to a relatively intensive one of $\sim 55 \mathrm{kD}$ (Fig.1A). Upon immune precipitation, saliva samples showed extremely reduced enzyme activities (Fig. 1B). In contrast, specific activities of AGAL in these samples were not significantly influenced. The specificity of the determination of AGAL activity in saliva was demonstrated by the finding that the specific potent competitive AGAL inhibitor, 1-deoxygalactonojirimycin hydrochloride, inhibited the enzyme reaction by nearly $100 \%$ at a concentration of $0.011 \mathrm{mM}$ (data not shown). The specific activity of IDUA was not affected by this compound. When expressed on a volume basis, the levels of IDUA (median value: $12.5 \mathrm{nmol}$ 4MU/h.ml) and AGAL (median value: $9.2 \mathrm{nmol}$ 4MU/h. 
$\mathrm{ml}$ ) activities in saliva of control subjects were even higher than those found in DBS (4.7 and $5.5 \mathrm{nmol} 4 \mathrm{MU} / \mathrm{h} . \mathrm{ml}$, respectively). These results prove that IDUA activity can be readily measured in saliva.
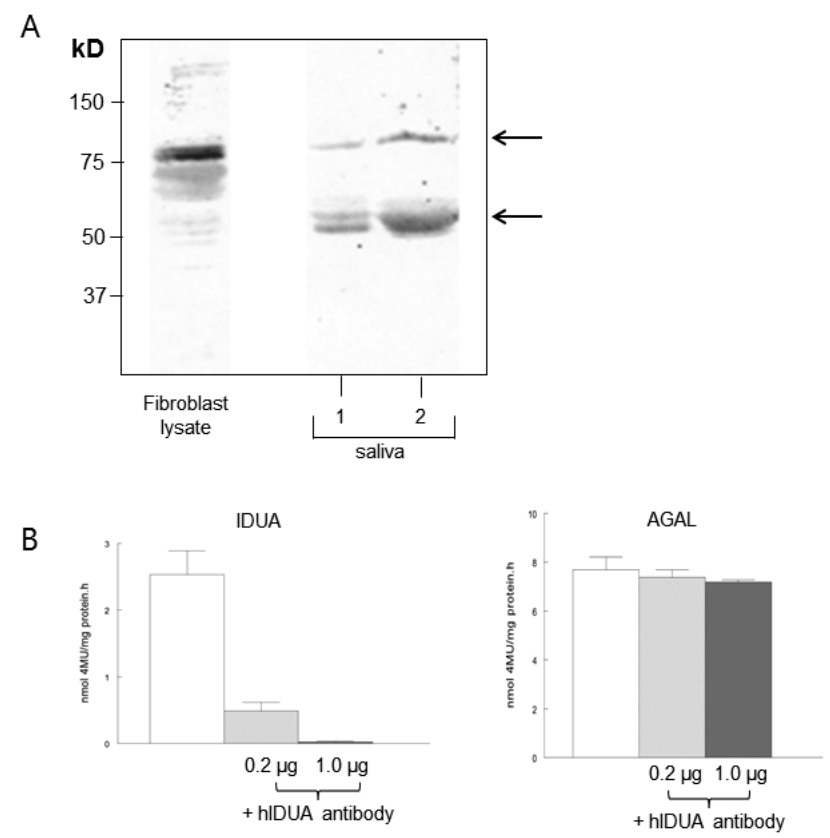

Figure 1. A: Western blotting of a lysate of cultured human fibroblasts and two samples of saliva from healthy subjects. Each sample was electrophoresed through 10\% SDS-poly acryl amide gels and blotted on Immobilon-FL PVDF. Blots were analyzed for IDUA using sheep serum anti-hIDUA .

B: Representative example of the determination of the specific activities of IDUA and AGAL in saliva after immunoprecipitation of IDUA with two different concentrations of antibody against hIDUA. White bar shows the specific activities of both enzymes in similarly processed saliva after incubation without antibody. Data are the mean \pm SD of measurements in triplicate.

\section{Storage of saliva}

As investigated for 3 healthy subjects, the specific activities of IDUA and AGAL, and the IDUA/AGAL ratio determined at different time points during the day (at 9.00h, 13.00h, $15.00 \mathrm{~h}$ and $17.00 \mathrm{~h}$ ) showed minor irregular fluctuations, with coefficients of variation (mean $\pm \mathrm{SD}$ ) of $21.2 \pm 0.9,20.2 \pm 11.1$, and $23.6 \pm 9.9 \%$, respectively. When immediately kept at $-80 \mathrm{oC}$ after collection, saliva specimens $(n=7)$ did not show significant changes in enzyme activities during at least 21 days. During storage at higher temperatures, both IDUA and AGAL appeared to be inactivated at a rather high rate $(\sim 17 \%$ and $40 \%$ after 7 days, for IDUA and AGAL respectively at $-20 \mathrm{oC}$, and $\sim 16 \%$ and $\sim 70 \%$ after 1 day at $4 \mathrm{oC}$ or room 
temperature). Addition of several protease inhibitors to the saliva samples prevented the decrease in enzyme activities during storage for 4 days at $4 \mathrm{oC}$ and room temperature by only $10 \%$ and $29 \%$, respectively.

\section{Diagnostic value of salivary IDUA activity}

The IDUA activity in saliva samples of untreated MPS-1H patients was very low, much further below the normal range (0.015-0.03 nmol 4MU/h.mg vs. 2.2-29.6 nmol 4MU/h.mg; $\mathrm{p}<0.0001)$ and also when compared to corresponding DBS $(0.4-0.5 \mathrm{nmol} 4 \mathrm{MU} / \mathrm{h} . \mathrm{ml}$ vs. 1.7$14.3 \mathrm{nmol} 4 \mathrm{MU} / \mathrm{h} . \mathrm{ml}$ (fig.2A and summarizing table S1). Furthermore, heterozygotes for MPS-1 were easily identified in saliva (0.9-5.1 nmol/h.mg, $\mathrm{p}<0.0001$; suppl. fig.1). Specific activities of AGAL in both DBS and saliva of patients, heterozygotes, and controls were similar (suppl. fig. 2A). Interestingly, patients with the milder MPS-1H/S phenotype showed distinctive and significant higher levels of salivary IDUA activity than the severe MPS-1H phenotype (0.06-0.51 nmol 4MU/h.mg vs. 0.0024-0.05 nmol 4MU/h.mg; p=0.046; fig. 2B). When IDUA activity in DBS and saliva was normalized towards the corresponding AGAL activities (IDUA/AGAL activity ratios), this largely resembled the pattern seen for the activities of IDUA (suppl. fig. 2B). These data indicate that measuring IDUA activity in whole saliva is a powerful method for the detection of IDUA deficiency and may even allow distinction between subtypes.

\section{Effect of HCT on IDUA activity}

Salivary IDUA activity levels in MPS-1H patients after HCT remained far below the normal range (0.02-0.3 nmol 4MU/h.mg vs. 2.2-29.6 nmol 4MU/h.mg; $\mathrm{p}<0.0001$; fig $2 \mathrm{~A}$ and table $\mathrm{S} 1)$. In contrast, DBS of all the MPS-1H patients who received HCT exhibited IDUA activity values within the normal range (1.7-14.3 nmol 4MU/h.ml; $\mathrm{p}=0.54$; fig. 2A). Similarly, AGAL activity values in DBS (3.7-17.3 nmol 4MU/h.ml) and saliva (2.2-18.7 nmol 4MU/h.mg) of all individual HCT MPS-1H patients lie within the normal range (1.4-31.8 nmol 4MU/h.ml and 1.4-17.3 nmol 4MU/h.mg, respectively); suppl. fig. 2). All 5 MPS-1H patients on ERT, showed subnormal activities of IDUA in their saliva, and 4 of them also in corresponding DBS samples. (fig. 2B).

Although salivary IDUA levels remain very low after HCT, there was a significant but variable increase after $\geq 6$ months when compared with the levels before $\mathrm{HCT}$ ( $p=0.031$; fig. $2 \mathrm{C}$ and table $\mathrm{S} 1$ ). This is similar in untreated MPS-1H and MPS-1H patients on ERT before HCT. IDUA activity levels remained low in the years after HCT (data not shown). 

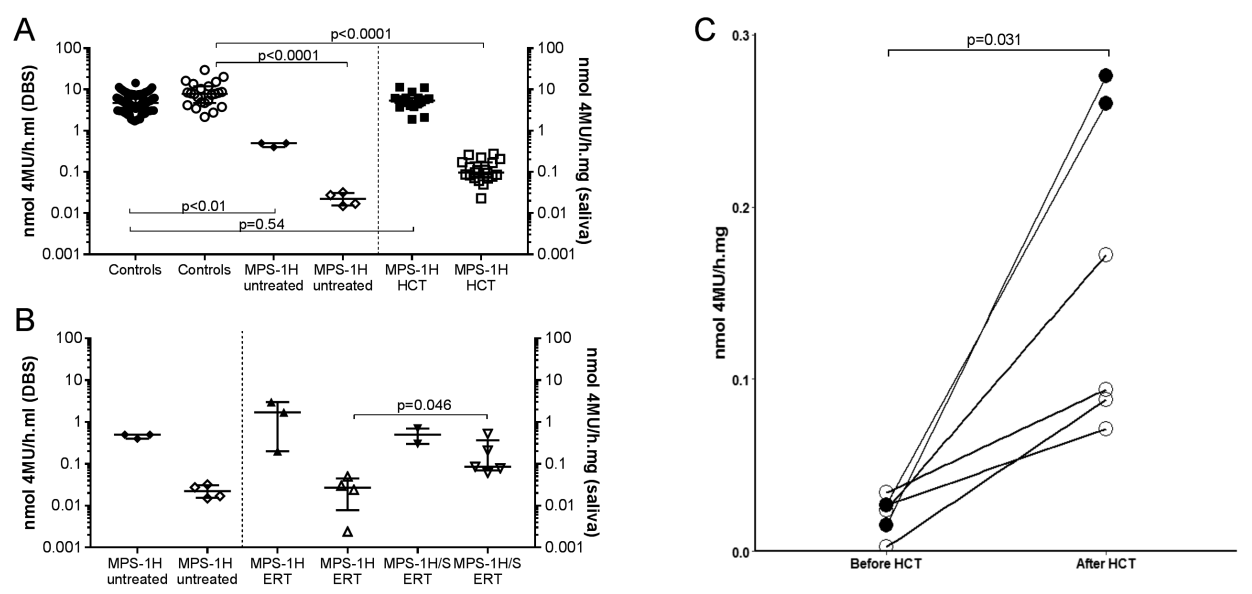

Figure 2. A: IDUA activities in DBS (closed symbols) and saliva (open symbols) in controls $(\mathbf{n}=116$ and $\mathbf{n}=\mathbf{2 3}$, respectively), untreated MPS-1H ( $n=3$ and $n=4)$, and MPS-1H patients after HCT $(n=18$ and $n=20)$. Salivary IDUA activity of untreated MPS-1H patients showed a significant difference with controls $(\mathrm{p}<0.0001)$. After HCT, IDUA activity is normalized in DBS $(\mathrm{p}=0.54)$ and remains significantly lower in saliva $(\mathrm{p}<0.0001)$.

B: IDUA activities in DBS (closed symbols) and saliva (open symbols) in untreated MPS-1H patients ( $n=3$ and $n=4$, respectively), MPS-1H patients on ERT $(n=3$ and $n=4)$, and MPS-1H/S patients on ERT ( $n=2$ and $n=5)$. MPS-1H/S patients showed significantly higher levels of salivary IDUA activity compared with MPS-1H patients $(\mathrm{p}=0.046)$. MPS-1H patients on ERT showed similar salivary IDUA activity as untreated patients $(\mathrm{p}=0.75)$.

$\mathrm{C}$ : Spaghetti plot of the activity of IDUA in saliva of paired samples of MPS-1H patients before and at least 6 months after treatment with HCT. Closed symbols depict untreated MPS-1H patients, open symbols depict MPS-1H patients on ERT before HCT. All patients showed an increase in specific IDUA activity ( $\mathrm{p}=0.031$; followup range: 0.5-3.89 years).

\section{DISCUSSION}

Despite normal levels of IDUA activity in either DBS, plasma or leukocytes after HCT, residual disease burden is still present in all patients with MPS-1H. To the best of our knowledge, this is the first study to investigate the potency of salivary IDUA activity to monitor the effect of treatment after HCT. Our results indicate measuring salivary IDUA activity is not only feasible but even represents a powerful method for the diagnosis of $\alpha$-iduronidase deficiency. More interestingly, while all MPS-1 patients exhibited normal IDUA enzyme activities in DBS after HCT, levels in saliva remained far below the normal range.

The observation that levels in saliva from patients after successful HCT remained far below the normal range, is in sharp contrast to the normalization of IDUA enzyme activity in DBS. The poor recovery of IDUA activity in saliva after treatment suggests the presence of a blood-saliva barrier, analogous to the blood-brain-barrier. This may reflect the situation in 
several other tissues, which are known to respond poorly to treatment with ERT or HCT (e.g. the cartilage and bone tissue). ${ }^{6,16}$ Lentiviral hematopoietic stem cell gene therapy is a novel therapy that aims for supra-normal hematological enzyme levels. ${ }^{17}$ Assessment of saliva IDUA activity might delineate to what extent this also provides better penetration in non-hematological compartments and could thus be used as a tool for monitoring the effect of new therapies.

In contrast to the brain, where the blood-brain-barrier is a well-known obstacle, no barriers were assumed for the transportation of IDUA in saliva, especially when considering the high degree of vascularization of the oral cavity. When revisiting Frantatoni's experiments, on which the principle of HCT is based, cross-correction resulted in normalization of intracellular storage products, suggesting enzyme import, without assessing this directly. ${ }^{18}$ Our data are in line with experimental data in mouse models that show that only a small amount of enzyme is enough to reduce intracellular storage products. It should be stressed that since we determine IDUA activity in saliva this reflects excretion and may be different from the enzyme levels in the salivary glands from which they originate. We propose that enzyme deficient cells of the salivary glands will excrete less enzyme compared to normal cells, which could explain the low levels of enzyme in saliva even after treatment with HCT. Buccal swab enzyme analysis of epithelial tissue could perhaps provide more information about what actually happens in the cells. However, the small increase after HCT could also be the result of donor macrophages who have migrated to the tissue. Insights in the remaining question why the penetration of IDUA activity in saliva of MPS-1 patients via the blood compartment by HCT is rather poor, could possibly lead to new therapeutic modalities.

This study also showed that the measurement of the IDUA specific activity in whole saliva is a powerful method for the diagnosis of IDUA deficiency. The collection of saliva offers a non-invasive and stress-free alternative compared to the collection of serum, urine or tissue biopsies. Although saliva is a complex fluid consisting of secretions of the major and minor salivary glands and various other components, ${ }^{7,19}$ it has been proven useful for diagnosis and monitoring of a variety of oral and systemic diseases. ${ }^{7,8,19}$ In lysosomal storage diseases however, this has not been studied so far. Interestingly, patients with the milder MPS-1H/S phenotype showed significantly higher levels of salivary IDUA activity than the severe MPS$1 \mathrm{H}$ phenotype and may thus allow distinction between subtypes, while this was not possible with the established blood testing. A larger cohort should elucidate whether saliva is useful in discriminating between the different MPS-1 phenotypes. In countries where MPS-1 is incorporated in the newborn screening program, it could then help clinicians decide whether a patient could be treated with ERT or is in need of HCT. 
A limitation of this study was the inter-individual variability of the saliva measurements. To ensure the requirements for reliability of both assay methods, a number of technical details were investigated, assuring that intrinsic artifacts were not introduced by our methods. Western blots of saliva, however, showed two IDUA immunoreactive bands. Possibly, hIDUA is present in different molecular weight forms, as reported previously. ${ }^{20}$ Finally, an important requirement for adequate results was rapid processing and storage of the saliva samples at $-80 \mathrm{o} C$ until analysis. During storage at higher temperatures both IDUA and AGAL appeared to be inactivated, despite the addition of several protease inhibitors. The cause of the apparent temperature induced inactivation of salivary IDUA and AGAL thus far remains enigmatic.

In conclusion, we show for the first time that IDUA activity can be reliably measured in saliva, allowing us to distinguish between patients with MPS-1 and controls, and even between MPS-1 subtypes. Moreover, measurements in patients with MPS-1H after HCT unveil that IDUA activity in saliva after HCT- contrary to the normalization observed in blood- remains very low. This may very well reflect the situation across vascularized non-hematological tissues and pinpoints enzyme delivery to these tissues as a weak spot of the current therapy. We propose that salivary IDUA activity could be used as a biomarker for the evaluation of the effect of new therapies in well vascularized non-hematological tissues.

\section{ACKNOWLEDGEMENTS}

All authors would like to thank all parents and patients for participating in this study. The authors wish to thank Dr. JJM Jans (Department of Genetics, University Medical Centre Utrecht) for critical reading of the manuscript. 


\section{REFERENCES}

1. Clarke LA. Mucopolysaccharidosis Type I. In: Pagon RA, Adam MP, Ardinger $\mathrm{HH}$, et al., eds. GeneReviews(R). Seattle (WA): University of Washington, Seattle. GeneReviews is a registered trademark of the University of Washington, Seattle. All rights reserved; 1993.

2. Wraith JE and Jones S. Mucopolysaccharidosis type I. Pediatr Endocrinol Rev. 2014; 12: 102-106.

3. Hobbs JR, Hugh-Jones K, Barrett AJ, et al. Reversal of clinical features of Hurler's disease and biochemical improvement after treatment by bone-marrow transplantation. Lancet. 1981; 2: 709-712.

4. Aldenhoven M, Boelens JJ, de Koning TJ. The clinical outcome of Hurler syndrome after stem cell transplantation. Biol Blood Marrow Transplant. 2008; 14: 485-498.

5. Jameson E, Jones S, Remmington T. Enzyme replacement therapy with laronidase (Aldurazyme((R))) for treating mucopolysaccharidosis type I. Cochrane Database Syst Rev. 2016; 4: CD009354.

6. Aldenhoven M, Wynn RF, Orchard PJ, et al. Long-term outcome of Hurler syndrome patients after hematopoietic cell transplantation: an international multicenter study. Blood. 2015; 125: 2164-2172.

7. Malamud D. Saliva as a diagnostic fluid. Dent Clin North Am. 2011; 55: 159-178.

8. Proctor GB. The physiology of salivary secretion. Periodontol 2000. 2016; 70:11-25.

9. Chojnowska S, Zalewska A, Knas M, et al. Determination of lysosomal exoglycosidases in human saliva. Acta Biochim Pol. 2014; 61: 85-90.

10. Den Tandt WR and Jaeken J. Determination of lysosomal enzymes in saliva. Confirmation of the diagnosis of metachromatic leukodystrophy and fucosidosis by enzyme analysis. Clin Chim Acta. 1979; 97: 19-25.

11. Zalewska A, Knas M, Niczyporuk M, et al. Salivary lysosomal exoglycosidases profiles in patients with insulin-dependent and noninsulin-dependent diabetes mellitus. Adv Clin Exp Med. 2013; 22: 659-666.

12. Chamoles NA, Blanco MB, Gaggioli D, Casentini C. Hurler-like phenotype: enzymatic diagnosis in dried blood spots on filter paper. Clin Chem. 2001; 47: 2098-2102.

13. Chamoles NA, Blanco M, Gaggioli D. Fabry disease: enzymatic diagnosis in dried blood spots on filter paper. Clin Chim Acta. 2001; 308: 195-196.

14. Chamoles NA, Niizawa G, Blanco M, Gaggioli D, Casentini C. Glycogen storage disease type II: enzymatic screening in dried blood spots on filter paper. Clin Chim Acta. 2004; 347: 97-102. 
15. Lowry OH, Rosebrough HJ, Farr AL, Randall RJ. Protein measurement with the Folin phenol reagent. J Biol Chem. 1951; 193: 265-275.

16. Passage MB, Krieger AW, Peinovich MC, et al. Continuous infusion of enzyme replacement therapy is inferior to weekly infusions in MPS I dogs. $J$ Inherit Metab Dis. 2009; 32: S253-8.

17. Visigalli I, Delai S, Politi LS, et al. Gene therapy augments the efficacy of hematopoietic cell transplantation and fully corrects mucopolysaccharidosis type I phenotype in the mouse model. Blood. 2010; 116: 5130-5139.

18. Fratantoni JC, Hall CW, Neufeld EF. Hurler and Hunter syndromes: mutual correction of the defect in cultured fibroblasts. Science. 1968; 162: 570-572.

19. Malathi N, Mythili S, Vasanthi HR. Salivary diagnostics: a brief review. ISRN Dent. 2014; $2014: 158786$.

20. Schuchman EH, Guzman NA, Desnick RJ. Human alpha-L-iduronidase. I. Purification and properties of the high uptake (higher molecular weight) and the low uptake (processed) forms. J Biol Chem. 1984; 259: 3132-3140. 


\section{SUPPLEMENTAL FIGURES}

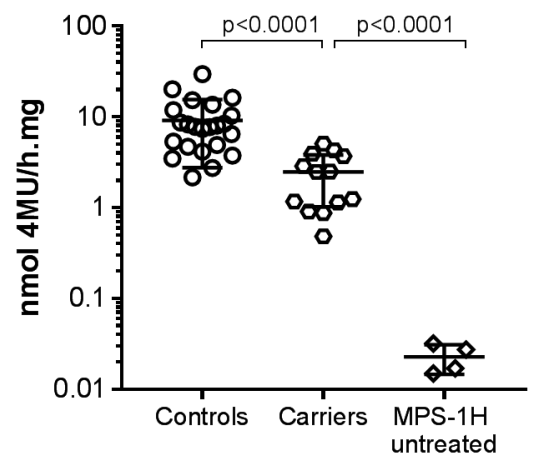

Suppl. figure 1. IDUA activity in saliva for controls $(n=23)$, carriers $(n=13)$, and MPS-1H untreated patients $(n=4)$. Untreated patients showed a significant difference with both carriers $(p<0.0001)$ and controls $(\mathbf{p}<0.0001)$. Furthermore, carriers showed a significant difference with both carriers $(\mathrm{p}<0.0001)$ and controls $(p<0.0001)$. Furthermore, carriers showed a significant difference with controls $(p<0.0001)$.
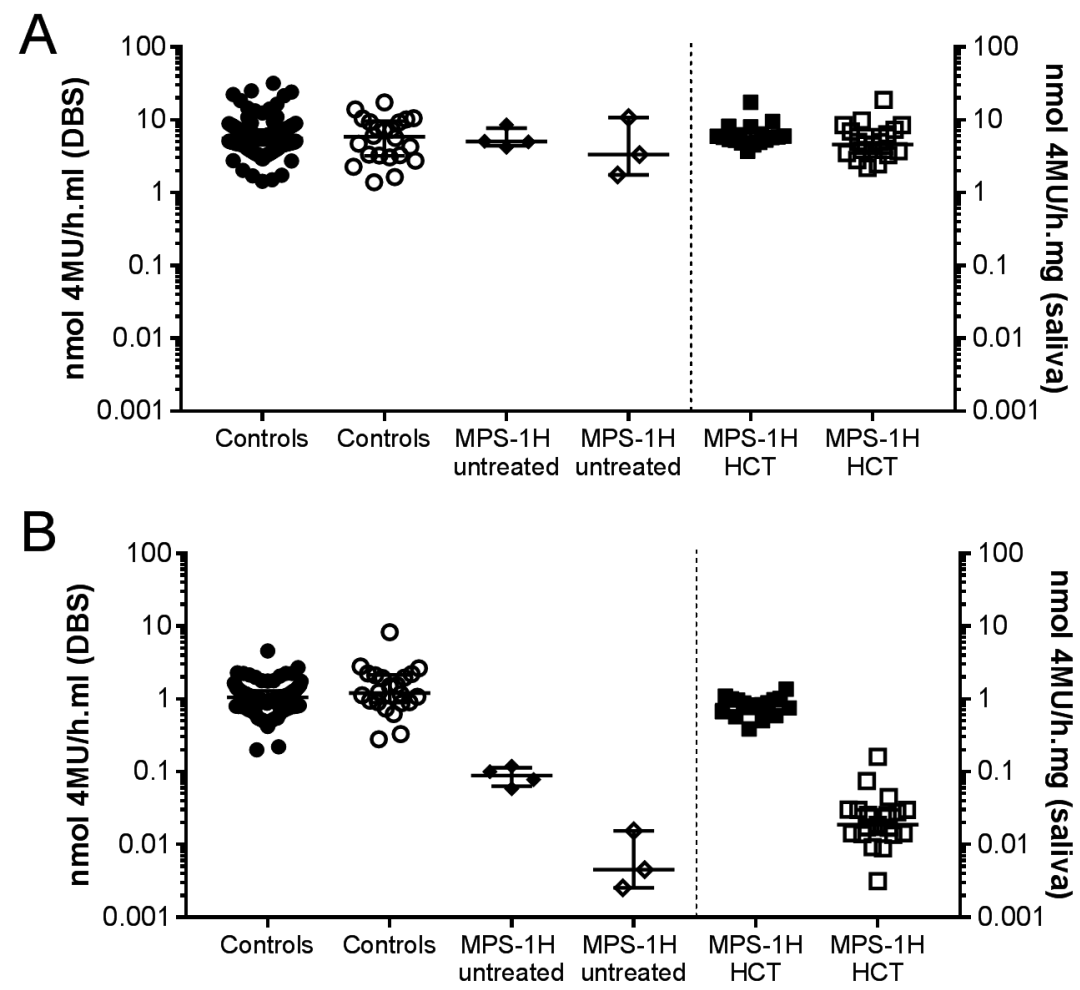

Suppl. figure 2. A: AGAL activity in DBS (closed symbols) and saliva (open symbols) in controls ( $\mathrm{n}=162$ and $n=22$, respectively), untreated MPS-1H ( $n=4$ and $n=3)$, and MPS-1H patients after HCT ( $n=18$ and $n=19)$.

B: IDUA/AGAL ratios in DBS (closed symbols) and saliva (open symbols) in controls ( $\mathrm{n}=99$ and $n=22$, respectively), untreated MPS-1H ( $n=4$ and $n=3)$, and MPS-1H patients after HCT $(n=18$ and $n=19)$. 


\section{SUPPLEMENTAL TABLES}

Suppl. table 1. Diagnostic and effect of therapy monitoring properties of IDUA activity in DBS versus saliva.

\begin{tabular}{lll}
\hline & DBS & Saliva \\
\hline Diagnosis & & \\
IDUA-deficiency & +++ & ++++ \\
Heterozygotes & $?$ & + \\
Classification & & \\
Hurler vs. Hurler-Scheie & - & ++ \\
Effect of therapy & \\
$\begin{array}{l}\text { Hematological } \\
\text { Potentially non-hematological }\end{array}$ & +++ \\
\hline DBS, dried blood spot; IDUA, alpha-1-iduronidase & - & ++ \\
\hline
\end{tabular}




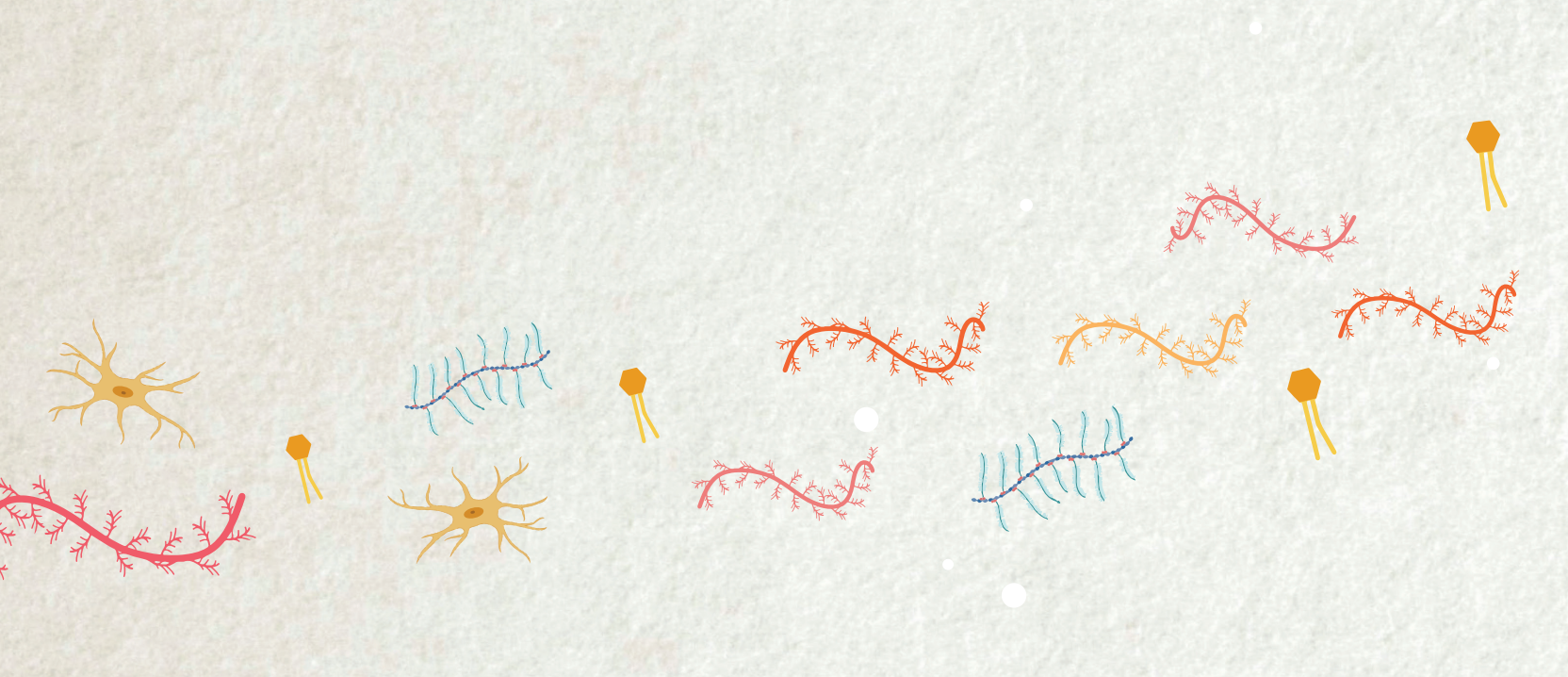



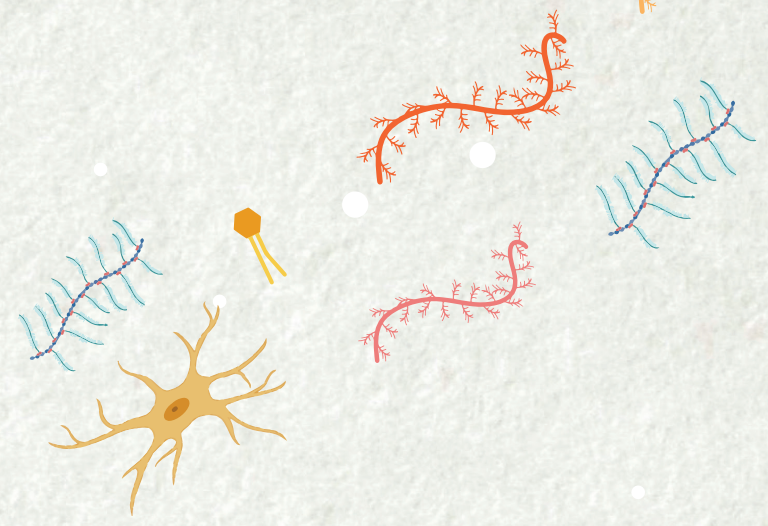

\section{CHAPTER 8}

\section{Long-Term Effect of Hematopoietic Cell Transplantation on Systemic Inflammation in Mucopolysaccharidoses Patients}

Brigitte T.A. van den Broek

Jaap Jan Boelens

Eveline M. Delemarre

Julia Drylewycz

Peter M. van Hasselt

Stefan Nierkens 


\section{ABSTRACT}

Background: Mucopolysaccharidoses (MPS) are devastating inherited diseases treated with hematopoietic cell transplantation (HCT). However, disease progression, especially skeletal, still occurs in all patients. Secondary inflammation has been hypothesized to be a cause.

Purpose: To investigate whether systemic inflammation is present in untreated patients and to evaluate the effect of HCT on systemic inflammation.

Methods: Dried blood spots of MPS patients $(n=30)$ treated with HCT between 2004 and 2019 were included. Time points consisted of pre-HCT, and at 1, 3, and 10 years of followup. 92 markers of the OLINK Inflammation I panel were measured and compared to agematched controls $(n=31)$ with principal component analysis and Wilcoxon rank-sum tests with correction.

Results: 69 samples of 35 patients were included. Median age at transplantation was 1.3 years (range $0.2-4.8$ years) and median time of pre-HCT sample to transplantation was 0.1 years. Normal leukocyte enzyme activity levels were achieved in $93 \%$ of patients postHCT. Pre-transplant samples showed clear separation of patients and controls. Markers that differentiated pre-HCT between controls and patients were mainly pro-inflammatory $(50 \%)$ or related to bone homeostasis and extracellular matrix degradation (33\%). At ten years of follow-up, only 5 markers, RANKL, OPG, AXIN1, SCF, and Flt3L, were still different between patients and controls.

Conclusion: Systemic inflammation is present in untreated MPS patients and is reduced upon treatment with HCT. Markers related to bone homeostasis remain elevated up to 10 years post-HCT and possibly reflect the ongoing skeletal disease making them potential biomarkers for the evaluation of new therapies. 


\section{INTRODUCTION}

The Mucopolysaccharidoses (MPS) are a group of diseases caused by genetic defects in the production of lysosomal enzymes which degrade glycosaminoglycans (GAGs). ${ }^{1}$ Without treatment, the consequences of these devastative multi-systemic disorders often result in premature death. Allogeneic hematopoietic cell transplantation (HCT) has shown to be a treatment option for a selected group of MPS patients. Intense international collaboration has resulted in strongly improved 5-year survival rates which now exceed $90 \%$ and positively changed long-term perspectives. ${ }^{2,3}$

Although HCT is a successful treatment in terms of long-term survivorship, the magnitude of residual disease is increasingly recognized..$^{4-6}$ The residual disease burden is multi-faceted, often involving several organ systems, including the skeleton, ligaments, eyes, and heart valves. Known predictors of the effect of therapy on long term outcomes are the hematological enzyme activity level after HCT (the higher the better) and the timing of therapy (the earlier the better). ${ }^{4}$ At present, except for the hematological enzyme activity level achieved after HCT, no biochemical markers are known to predict the long-term outcome. Currently, improved treatment protocols have resulted in normal enzyme activity levels in the majority of patients and newborn screening programs to detect patients at an early age are evolving. Patients, however, although transplanted at an early age with normal post-HCT enzyme activity levels, still develop residual disease which has shown to progress over time.4-7 This has led to the development of new therapies, such as autologous gene-transduced cell transplantation aiming for supra-physiological hematologic enzyme activity levels to achieve higher penetrance in tissues, for which clinical trials are now open for MPS-1 (clinicaltrials. gov, NCT03488394) and MPS-3 (clinicaltrials.gov, NCT04201405) patients. Biochemical markers, however, that reflect residual disease and can potentially predict whether new therapies are better in treating all facets of the disease, are lacking.

Interest in the involvement of the immune system in the pathology of MPS has grown in the last years. Several studies in both mice and men have shown that the immune system in MPS patients is in a pro-inflammatory state. ${ }^{8-11}$ As inflammation is a secondary manifestation of the original disease, we hypothesized that systemic inflammation markers could be potential biomarkers for the evaluation of new therapies that aim to improve the long term clinical outcomes of MPS patients. Therefore, in this study, we investigated whether systemic inflammation was present in untreated patients and to what extent this is ongoing after HCT in the aim to provide a signature of residual disease that reflects the weak spots of current HCT treatment. 


\section{PATIENTS AND METHODS}

\section{Study participants}

All MPS patients treated with HCT in the Wilhelmina Children's Hospital (WKZ) of the University Medical Centre Utrecht (UMCU), The Netherlands between 2003 and 2019, were included in this study. All patients gave informed consent before joining the study and approval from to the responsible institutional committee Medical Research Involving Human Subjects was retrieved. The MPS phenotype, date of birth, date of sample collection, age at transplantation, leukocyte enzyme activity level before and after transplantation (scored as below or above lower limit of normal, cut-off point: $20 \mathrm{nmol} / \mathrm{h} / \mathrm{mg}$ ), and use of medication were collected from the medical records. As controls, dried blood spots (DBS) of stable phenylketonuria patients were collected in the age ranges 0-1 year, 1-3 years, 3-6 years, 6-12 years, and $>12$ years.

\section{Dried blood spot sample preparation}

DBS of MPS patients at baseline (pre-HCT), 1 year, 3 years, and 10 years after HCT were collected. The use of corticosteroids or immunosuppressive drugs at time of collection excluded the sample for analysis. DBS were prepared by dispensing whole blood collected by vena puncture in a heparin tube or directly from a finger prick onto Guthrie cards. The DBS were subsequently dried for at least 4 hours at room temperature and stored at $-80^{\circ} \mathrm{C}$ in a plastic bag until analysis. No differences in markers were seen in DBS prepared from the heparin tubes compared to DBS prepared from a direct finger prick (data not shown).

\section{OLINK Multiplex assay}

Dried blood spots were analyzed with the OLINK Proseek(C) multiplex Inflammation I assay in the University Medical Center Utrecht, which is a proximity extension assay (PEA) technology with oligonucleotide-labeled antibody probe pairs that bind to their respective targets. ${ }^{12,13}$ From the DBS, $1.2 \mathrm{~mm}$ punches were obtained and $1 \mu \mathrm{l}$ sample diluent was added to the punches to compensate for volume. Hereafter, the process was performed following the manufacturer's manual. In short, PEA technology makes use of antibody pairs direct to specific proteins. Upon binding of antibody pairs to their respective targets, DNA reporter molecules bound to the antibodies give rise to new DNA amplicons with each ID-barcoding their respective antigens. The amplicons were subsequently quantified using a Fluidigm BioMark $^{\mathrm{TM}} \mathrm{HD}$ real-time PCR platform. The raw $\mathrm{Cq}$ values were normalized and converted into Normalized Protein Expression Units (NPX). The NPX values were expressed on a Log2 scale where one unit higher in NPX values represents a doubling of the measured protein concentration. Quality control of the measured samples was carried out using Olink's standard quality control protocol. The simultaneously measured 92 proteins are listed in 
suppl. table 1 . The assay was performed at the same time for all patients and controls.

\section{Statistical Analysis}

Baseline characteristics are reported as frequencies and percentages for categorical variables and median (range) for continuous variables. Two distinct datasets were obtained for patients with paired DBS and for patients with one DBS in addition of DBS collected from controls. Before analysis, the datasets were merged and the total dataset was corrected for batch effects using R package $\mathrm{SVA}^{14}$, function ComBat. ${ }^{15}$ To test whether inflammation was present in MPS patients compared to age-matched controls, analyses using a Principal Component Analysis (PCA). Furthermore, DBS analyte NPX levels between the patients and controls were compared using a Wilcoxon rank-sum test with Benjani-Hochberg correction for multiple comparisons. Finally, fold change analyses were performed on the linearized NPX values. To visualize the fold change values in a volcano plot, the fold change values were transformed to $\log 2$ fold change (data not shown). Markers that both showed a $\log 2$ fold change of $>1.5$ and were significant according to the Wilcoxon rank-sum test after false discovery rate correction were considered important in the comparison between patients and controls. Clustering for heatmaps was based on Ward's method and pairwise correlation distance.

To determine which markers showed a significant change over time, we used linear mixed effect models and used the fixed estimate for time of each marker as a proxy. Linear mixed models were fitted for the NPX values of each marker as dependent variables. Time was included as fixed effect. Initially, a random intercept was included per individual to account for individual variation at baseline and a random slope (for time) for dependency across the repeated measurements within the same individual during follow-up. Models that were not able to converge were subsequently fitted with a fixed effect for time in combination with a random intercept per individual without the random effect for time. Final coefficients were estimated using a restricted maximum likelihood approach. The model assumptions including normal distributed residuals, random effects, and homogeneity of variance were confirmed visually. The p-values for the fixed effect for time were extracted from the models and corrected with Benjani-Hochberg correction for multiple comparisons. Adjusted P-values $<0.05$ were considered significant. The R project (RStudio: Integrated Development for R. RStudio, Inc., Boston, MA) for statistical computing version 3.4.1 with the packages 'Ime4' and 'ggplot2' was used for all analyses and for the creation of figures. 


\section{RESULTS}

\section{Patient characteristics}

In total, 100 samples from both patients and controls were included in the analyses. Of these, 69 samples originated from 35 patients. Patients characteristics are shown in table 1. The majority of patients were diagnosed with MPS-1H $(n=24)$. Two patients were diagnosed with MPS-1H/S, two with MPS-2, two with MPS-3, two with MPS-4, and three with MPS-6. PreHCT samples were available from 22 patients. The median age at pre-HCT sample collection was 1.1 years (range 0.1 - 4.7 years). Forty-seven follow-up samples were available from 30 patients. The median age at transplantation was 1.3 years (range $0.2-4.8$ years). All but 2 patients (7\%) had enzyme activity levels in the normal range. At 1-year follow-up, 19 samples were available, at 3 years follow-up 18 samples, and at 10 years follow-up 10 samples. The remaining 31 samples originated from 31 controls with a median age of 9 years (range 0-29). For controls, 3 samples were available in the age range of 0-1 year, 6 samples in the range of 1-3 years, 4 samples in the range of 3-6 years, 8 samples in the range of 6-12 years, and, finally, 10 samples were collected from controls aged $\geq 12$ years.

Table 1. Patient characteristics and number of samples.

\begin{tabular}{lc}
\hline \multicolumn{2}{c}{ Patient characteristics $(\mathbf{n}=\mathbf{3 0})$} \\
\hline Gender (male) & $21(70 \%)$ \\
Age at pre-HCT collection (years; median, range) & $1.1(0.1-4.7)$ \\
Age at transplantation (years; median, range) & $1.3(0.2-4.8)$ \\
Enzyme activity level post-HCT (> normal range) & $28(93 \%)$
\end{tabular}

\begin{tabular}{ccccc}
\hline & \multicolumn{5}{c}{ DBS (n) } \\
\hline MPS & Pre & 1 year FU & 3 years FU & $\mathbf{1 0}$ years FU \\
MPS-1 & 22 & 19 & 18 & 10 \\
MPS-2 & 16 & 14 & 15 & 10 \\
MPS-3 & 2 & 2 & 1 & \\
MPS-4 & 2 & 2 & & \\
MPS-6 & 2 & 1 & 1 \\
\hline
\end{tabular}

NB: None used corticosteroids or immunosuppressive medication at sample collection

\section{Inflammation in untreated MPS patients}

Supervised analysis, comparing pre-HCT MPS patients $(n=22)$ with age-matched controls, resulted in a list of 64 markers after correction for multiple testing. When combing significance and fold change of $>2.7$, the list was reduced to 37 markers (data not shown). Unsupervised PCA revealed a clear separation of pre-HCT MPS patients versus age-matched controls with relatively higher DBS concentrations of markers in MPS patients than in controls. The first 
component described the majority of data set variance with a remarkable 52\% (figure 1a and figure 1b). Separate clustering between the different MPS-subtypes was not observed (figure 1a). A nearly perfect separation was also observed when the data was visualized in a heatmap based on hierarchical clustering for both samples and cytokines (suppl. figure 2).

A

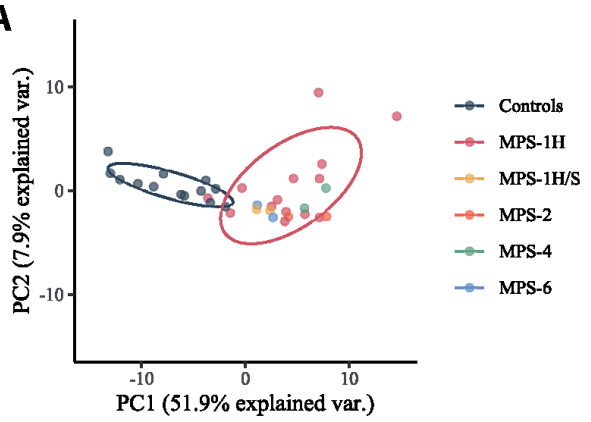

B

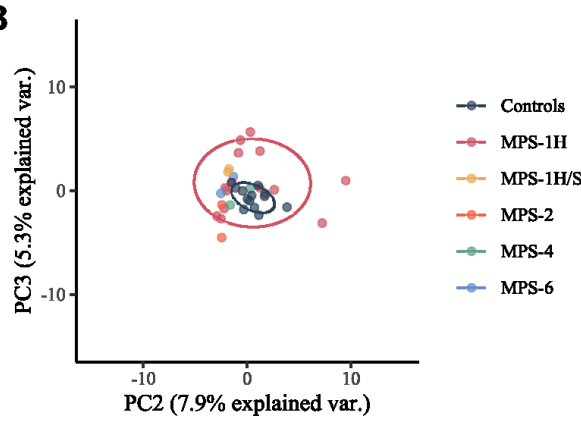

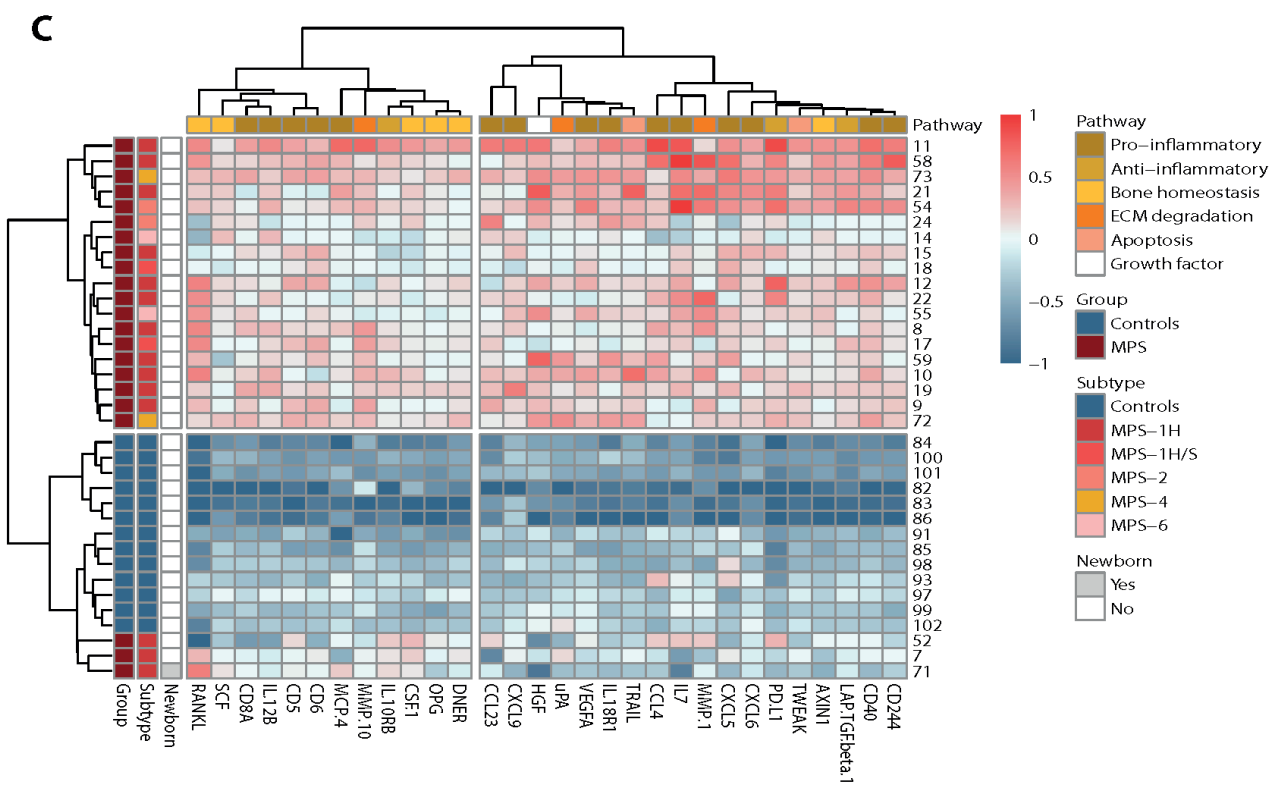

Figure 1. PCA and Heatmap of inflammation markers in untreated MPS patients versus controls. A: Principal component 1 versus component 2 shows clear separation of controls (blue) and MPS patients $(\mathrm{n}=22)$. B: Principal component 2 versus component 3. C: Heatmap of inflammation markers both present in component 1 of unsupervised PCA and list of significant and >1.5-fold change supervised analysis. On the left, color coding is shown for Group (controls vs. MPS), Subtypes (of the different MPS), and whether the patient was a newborn or not. The color coding on top shows to which pathway the different markers belong. 
Table 2. Selection of differentiating inflammation markers in untreated MPS patients versus controls. Markers that were present in both the list of markers that were significant and had a fold change $>2.7$ in the supervised analysis and the first component of unsupervised PCA. Markers are categorized based on their relation to MPS pathology.

\begin{tabular}{|c|c|c|c|}
\hline Marker & Eponyme & Pathway & Function \\
\hline CD5 & & Pro-inflammatory & Expressed on surface of T-cells. \\
\hline CD6 & & Pro-inflammatory & Expressed on surface of T-cells. \\
\hline CD8A & & Pro-inflammatory & T-cell surface glycoprotein, co-receptor. \\
\hline CD40 & & Pro-inflammatory & Co-stimulatory protein on APCs. \\
\hline CD244 & $\begin{array}{l}\text { NK cell } \\
\text { receptor } 2 \mathrm{~B} 4\end{array}$ & Pro-inflammatory & Cell surface receptor of NK cells (and T cells). \\
\hline CCL4 & MIP-1 $\beta$ & Pro-inflammatory & $\begin{array}{l}\text { Chemoattractant for NK cells and monocytes, produced } \\
\text { by neutorphils, monocytes, T cells, B cells, fibroblasts, } \\
\text { endothelial \& epithelial cells. Increased with age. }\end{array}$ \\
\hline CCL23 & MIP-3 & Pro-inflammatory & Chemotactic for resting T-cells and monocytes. \\
\hline CXCL5 & ENA78 & Pro-inflammatory & $\begin{array}{l}\text { Produced following stimulation of cells with IL1 or } \\
\text { TNF- } \alpha \text {, chemotactic for neutrophils. }\end{array}$ \\
\hline CXCL 6 & GCP-2 & Pro-inflammatory & Chemoattractant for neutrophils. \\
\hline CXCL9 & MIG & Pro-inflammatory & $\begin{array}{c}\text { Induces chemotaxis of CTLs, NKs, and macrophages, } \\
\text { promotes differentiation and multiplication of } \\
\text { leukocytes. Also causes tissue extravasation. }\end{array}$ \\
\hline IL7 & & Pro-inflammatory & $\begin{array}{l}\text { HGF secreted by stromal cells in BM and thymus. } \\
\text { Also produced by keratinocytes, DCs, hepatocytes, } \\
\text { neurons, epithelial cells, not produced by lymphocytes. } \\
\text { Stimulates differentiation of HSCs to myeloid } \\
\text { progenitor cells and proliferation of all cells in lymphoid } \\
\text { lineage (B/T/NK). }\end{array}$ \\
\hline IL.12B & & Pro-inflammatory & $\begin{array}{c}\text { Expressed by activated macrophages, acts on T and } \\
\text { NK cells, important for sustaining sufficient number of } \\
\text { memory/effector Th1 cells. }\end{array}$ \\
\hline IL.18R1 & & Pro-inflammatory & $\begin{array}{l}\text { Binds IL18, induced by IFN- } \alpha \text { and IL12 in NK and T } \\
\text { cells. }\end{array}$ \\
\hline MCP.4 & $\mathrm{CCl13}$ & Pro-inflammatory & $\begin{array}{l}\text { Induces chemotaxis in monocytes, eosinophils, T cells, } \\
\text { basophils. Induced by IL1 and TNF- } \alpha \text {. }\end{array}$ \\
\hline VEGFA & & Pro-inflammatory & $\begin{array}{l}\text { Found prominently on endothelial cell membrane, } \\
\text { also stimulates monocytes migration and increases } \\
\text { microvascular permeability upon binding to its receptors } \\
\text { (VEGFR1 and VEGFR2) and to heparan sulfate and } \\
\text { heparin. }\end{array}$ \\
\hline IL.10RB & & Anti-inflammatory & $\begin{array}{l}\text { Together with IL10RA required for IL10-induced signal } \\
\text { transduction. }\end{array}$ \\
\hline
\end{tabular}


LAP.TGF.beta. 1

Anti-inflammatory

PD.L1

CD274

Anti-inflammatory

RANKL

TRANCE /

TNFSF11

Bone homeostasis

OPG

TNFRSF11B Bone homeostasis

AXIN1

Bone homeostasis

SCF

KIT-ligand

Growth factor /

Bone homeostasis

CSF.1

DNER

Growth factor/

Bone homeostasis

TRAIL

TNFSF10

Apoptosis /

Bone homeostasis
Part of complex with latent TGF-binding protein and

TGF $\beta$. Cleaved by MMP-9 and MMP-2, TGF- $\beta$ induces development of regulatory $\mathrm{T}$ cells, has an inhibitory effect on B cell proliferation, stimulates resting monocytes and inhibits activated macrophages. Interacts with CXCL9, suppresses adaptive arm of immune system, reduces apoptosis in regulatory T-cells. Expressed on T cells, NK cells, macrophages, myeloid DCs, B cells, epithelial cells, vascular endothelial cells upon IFN- $\gamma$ stimulation.

Receptor activator of NF-kB ligand. Osteoclastic activity is triggered via the osteoblasts' surface-bound RANKL activating the osteoclasts' surface-bound RANK.

Osteoclastogenesis inhibitory factor and decoy receptor for RANKL. Also binds to TRAIL and inhibits TRAIL induced apoptosis. Glycosaminoglycans also are ligands for OPG. Expressed on osteoblast lineage cells. Expression is highly regulated by estrogens Upregulation of OPG suppresses osteoclastogenesis and bone resorption. Wnt and TGF- $\beta$ are OPG inducing cytokines.

Negative regulator of the Wnt pathway and can induce apoptosis.

Cytokine that binds to c-KIT receptor (CD117) and is important in the regulation of HSCs in the stem cell niche in the bone marrow. It contributes to the self-renewal and maintenance of HSCs. Stromal cell surrounding HSCs release SCF. HSCs become less sensitive to SCF in adulthood.

Hematopoietic growth factor, differentiates HSCs into macrophages, drives proliferation of a pro-inflammatory macrophage phenotype in acute kidney injury. Also released by osteoblasts, binds on osteoclasts inducing differentiation.

Delta/Notch-like epidermal growth factor-related receptor is an activator of the NOTCH1 pathway. Produced by most normal tissue cells and induces caspase-8-dependent apoptosis after binding to death receptors DR4 and DR5. Also ligand for DcDR1 (TRAIL-neutralizing decoy-receptor), DcDR2 (activates NF-kB pathway), and OPG. TRAIL induces osteoclastogenesis by binding to specific TRAIL receptors on osteoclast precursor cell surfaces 


\begin{tabular}{|c|c|c|c|}
\hline TWEAK & TNFSF12 & Apoptosis & $\begin{array}{l}\text { Ligand for TWEAKR receptor and can induce apoptosis } \\
\text { via multiple pathways. Produced mainly by leukocytes, } \\
\text { including monocytes, DCs, and NK cells. }\end{array}$ \\
\hline MMP.1 & & ECM degradation & $\begin{array}{l}\text { MMP enzyme in ECM remodeling, targets collagens } \\
\text { types I, II, and III. HGF activates MMP-1 activity via } \\
\text { the Etsy transcription family. }\end{array}$ \\
\hline MMP.10 & & ECM degradation & $\begin{array}{c}\text { MMP enzyme in ECM remodeling, targets a.o. } \\
\text { proteoglycans, cleaves precursor of MMP.1 }\end{array}$ \\
\hline uPA & & ECM degradation & $\begin{array}{l}\text { Urokinase plasminogen activator, primary substrate } \\
\text { is plasminogen (precursor of plasmin). Activation } \\
\text { of plasmin can trigger a proteolytic cascade that } \\
\text { participates in thrombolysis or extracellular matrix } \\
\text { degradation. uPA also can cleave pro-HGF. Ligand for } \\
\text { uPAR, expressed by both osteoblasts and osteoclasts }\end{array}$ \\
\hline HGF & SF & $\begin{array}{l}\text { ECM degradation / } \\
\text { Growth factor }\end{array}$ & $\begin{array}{l}\text { Paracrine cellular growth, motility, and morphogenic } \\
\text { factor secreted by mesenchymal cells as pro-HGF which } \\
\text { is a.o. activated by uPA. Primarily targets epithelial } \\
\text { and endothelial cells, but also haemopoietic progenitor } \\
\text { cells and T cells. HGF upregulates the expression of } \\
\text { IL11 from osteoclast-like cells and inhibits osteoblast } \\
\text { differentiation. HGF increases production of MMP-1 } \\
\text { and uPA. Has an anti-fibrotic effect in various organs. }\end{array}$ \\
\hline
\end{tabular}

Markers that were both present in the list of 37 differentiating markers in the supervised analysis and the top 40 markers of the first component of the unsupervised PCA were selected for further analysis and are shown in table 2 and figure $1 \mathrm{c}(\mathrm{n}=30)$. Of these, an interesting set of ten markers was associated with bone homeostasis $(n=6 ; 20 \%)$ and ECM degradation $(n=4 ; 13 \%)$. Furthermore, the majority of markers $(n=15 ; 51 \%)$ was associated with a proinflammatory immune status. The other markers were associated with an anti-inflammatory status $(n=3 ; 10 \%)$, apoptosis $(n=1 ; 3 \%)$, or were growth factors $(n=1 ; 3 \%)$.

\section{Inflammation in MPS patients treated with HCT}

To reveal what effect HCT has on the inflammation status of MPS patients, a longitudinal analysis was performed. Of the 92 measured markers, a total of 12 markers changed significantly over 10 years' time and are shown in table 3. Three of these, CCL4, CXCL9, and SCF, were also important in the differentiation between pre-HCT patients and controls. Because no longitudinal data of controls were available, these were not included in the longitudinal analyses. In the cross-sectional data of controls, only one marker, CCL28, was found to be statistically different between the predefined age groups (0-1 year, 1-3 years, 3-6 years, 6-12 years, and $>12$ years). However, although not statistically significant, when visualized, the majority of markers, did show a trend to increase over time in controls (suppl. figure 3). 
Table 3. Markers with significant change over time

\begin{tabular}{ccccc}
\hline Marker & Slope & P-value & Pathway & $\begin{array}{c}\text { Differentiating } \\
\text { pre-HCT }\end{array}$ \\
\hline CXCL9 & -0.09 & 0.049 & Pro-inflammatory & Yes \\
CXCL10 & -0.12 & 0.04 & Pro-inflammatory & No \\
CCL3 & -0.13 & 0.01 & Pro-inflammatory & No \\
CCL4 & -0.10 & 0.02 & Pro-inflammatory & Yes \\
CCL19 & -0.09 & 0.03 & Pro-inflammatory & No \\
CCL20 & -0.09 & 0.04 & Pro-inflammatory & No \\
CCL25 & -0.08 & 0.02 & Pro-inflammatory & No \\
MCP.3 & -0.07 & 0.03 & Pro-inflammatory & No \\
IFN.gamma & -0.12 & 0.04 & Pro-inflammatory & No \\
TNF & -0.07 & 0.009 & Pro-inflammatory & No \\
TNFB & -0.06 & 0.04 & Pro-inflammatory & No \\
TNFRSF9 & -0.08 & 0.009 & Pro-inflammatory & No \\
CDCP1 & -0.06 & 0.04 & Pro-inflammatory & No \\
Flt3L & 0.1 & 0.03 & Growth factor & No \\
SCF & 0.1 & 0.03 & Growth factor & Yes \\
\hline
\end{tabular}

At three years post-HCT, when comparing cross-sectional data between patients and agematched controls, all markers that were pre-HCT differentiators remained to be present in both the list of markers that were significant with a fold change $>2.7$ between patients and controls and the top 40 markers of the first component of the PCA.

At 10 years' follow-up, when looking at significance only and thus without taking the fold change into account, 30 markers were still different between patients and age-matched controls (table 4). All of these were also significant pre-HCT. Only five markers, RANKL, OPG, AXIN1, SCF, and Ftl3L, remained elevated with a $>2.7$-fold change difference (figure 2). 
Table 4. Markers significantly different between MPS patients and age-matched controls at 10 years' follow-up.

\begin{tabular}{|c|c|c|c|}
\hline Marker & $\begin{array}{c}\text { Adjusted } \\
\text { P-value }\end{array}$ & $\begin{array}{c}\text { Fold } \\
\text { change }\end{array}$ & Pathway \\
\hline CCL19 & 0.04 & 1.4 & Pro-inflammatory \\
\hline CCL20 & 0.01 & 1.6 & Pro-inflammatory \\
\hline CD244 & 0.004 & 2.2 & Pro-inflammatory \\
\hline CD40 & 0.01 & 1.8 & Pro-inflammatory \\
\hline CD5 & 0.006 & 2.1 & Pro-inflammatory \\
\hline CD6 & 0.002 & 2.6 & Pro-inflammatory \\
\hline CD8A & 0.004 & 2.5 & Pro-inflammatory \\
\hline CDCP1 & 0.02 & 1.3 & Pro-inflammatory \\
\hline CX3CL1 & 0.01 & 1.7 & Pro-inflammatory \\
\hline CXCL5 & 0.004 & 2.3 & Pro-inflammatory \\
\hline IL.12B & 0.003 & 2.2 & Pro-inflammatory \\
\hline IL.18R1 & 0.03 & 1.7 & Pro-inflammatory \\
\hline IL7 & 0.001 & 2.0 & Pro-inflammatory \\
\hline МCP.2 & 0.02 & 1.6 & Pro-inflammatory \\
\hline MCP.4 & 0.008 & 1.7 & Pro-inflammatory \\
\hline TNFB & 0.05 & 1.4 & Pro-inflammatory \\
\hline LAP.TGF.beta. 1 & 0.03 & 1.6 & Anti-inflammatory \\
\hline IL.10RB & 0.01 & 1.7 & Anti-inflammatory \\
\hline RANKL & 0.001 & 2.7 & Bone homeostasis \\
\hline OPG & 0.001 & 2.8 & Bone homeostasis \\
\hline AXIN1 & 0.002 & 3.2 & Bone homeostasis \\
\hline SCF & 0.0005 & 3.5 & Growth factor / Bone homeostasis \\
\hline Flt3L & 0.0002 & 2.8 & Growth factor / Bone homeostasis \\
\hline CSF.1 & 0.004 & 2.2 & Growth factor / Bone homeostasis \\
\hline DNER & 0.001 & 2.5 & Growth factor / Bone homeostasis \\
\hline LIF.R & 0.006 & 1.5 & Growth factor \\
\hline TRAIL & 0.03 & 1.8 & Apoptosis / Bone homeostasis \\
\hline TWEAK & 0.001 & 2.3 & Apoptosis \\
\hline MMP.10 & 0.01 & 1.9 & ECM degradation \\
\hline
\end{tabular}



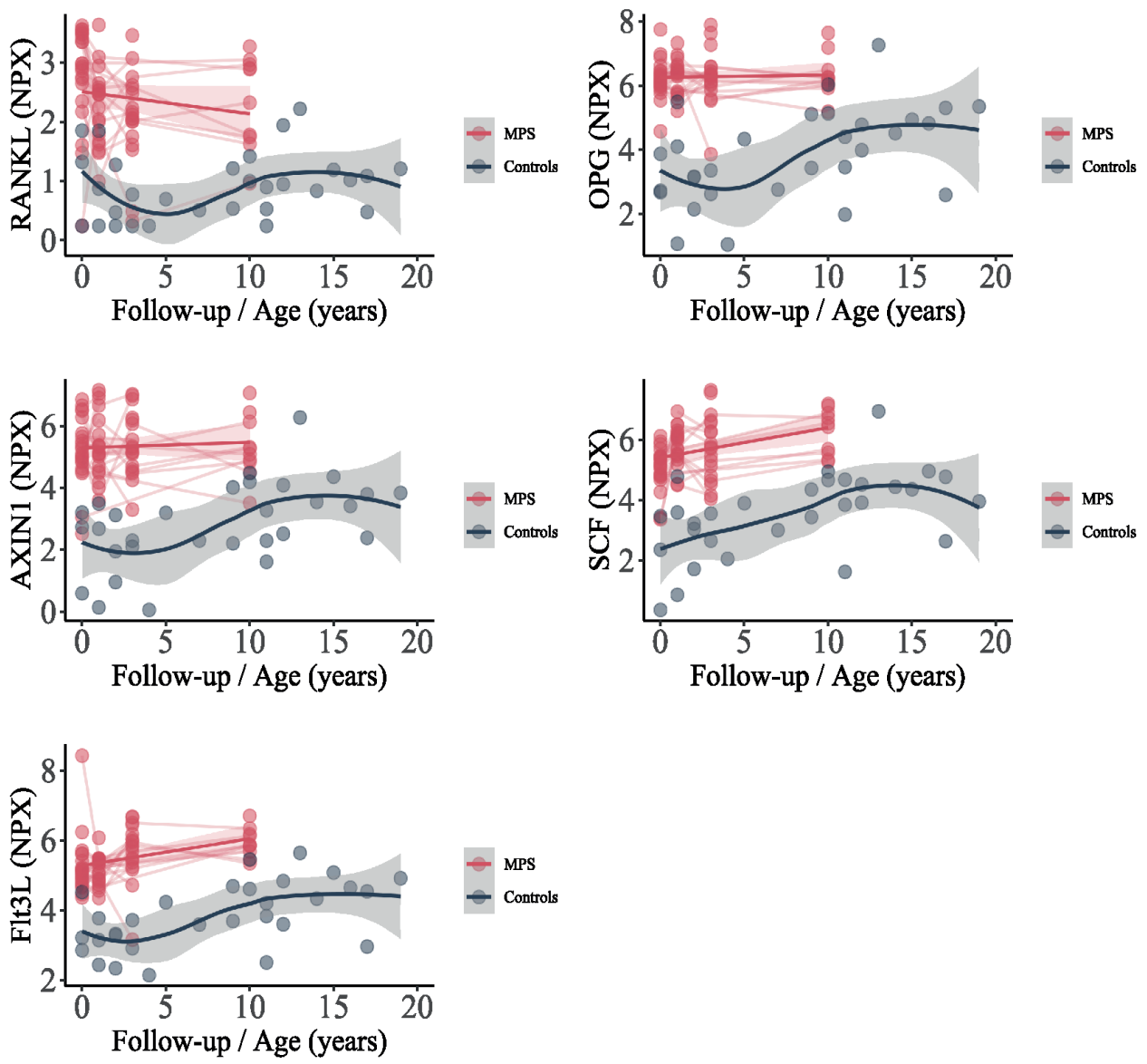

Figure 2. Differentiating markers at 10 years follow-up. Longitudinal data of patients are shown in red, crosssectional data of controls in blue. Average line is estimated by a linear mixed model for longitudinal patient data and shown with a 'Loess' curve for controls. X-axes show Follow-up (in years) for patients and Age (in years) for controls. Median age at transplantation was 1.3 years (range $0.2-4.8$ years).

\section{DISCUSSION}

This is the study to describe, the course of systemic inflammation in transplanted MPS patients with up to 10 years of follow-up. Before transplantation, a clear increase in a large profile of mainly pro-inflammatory biomarkers was seen in MPS patients compared to controls. Early after transplantation (up to three years), inflammation was still present in MPS patients compared to controls. However, at 10 years of follow-up, most of the inflammatory markers had decreased to (near) normal levels. Five markers, RANKL, OPG, AXIN1, Flt3L, and SCF, which are associated with bone homeostasis, remained significantly increased in combination 
with a fold change $>2.7$ compared to controls at 10 years after transplantation, suggesting they may serve as biomarkers for (skeletal) residual disease.

The pro-inflammatory profile seen before transplantation is in line with previous research. ${ }^{8-11,16}$ GAGs are direct inducers of the TLR-4 pathway resulting in the production of pro-inflammatory cytokines including TNF- $\alpha .{ }^{17}$ Simonaro et al. ${ }^{18}$ have demonstrated the importance of the TLR-4 pathway in the pathogenesis of cartilage and bone disease in MPS, and a pilot study with anti-TNF- $\alpha$ in MPS patients resulted in lower pain scores. ${ }^{19}$ However, anti-TNF- $\alpha$ treatment comes with disadvantages and side effects. Therefore, less toxic anti-inflammatory agents have been researched. One example is Pentosan polysulphate (PPS), which is a semi-synthetic sulfated polysaccharaide polymer with anti-coagulent, prochondral, and anti-inflammatory properties. In MPS animal models it has shown to reduce GAG concentrations and to improve cartilaginous and osseous pathology. ${ }^{20}$ A phase 1/II clinical trial in adult MPS-1 patients with the less severe phenotypes (Scheie and HurlerScheie) on enzyme replacement therapy (ERT), showed further reduction in urine GAG concentrations and improved range of motion after 24 weeks of PPS treatment. ${ }^{21}$ PPS has also been studied in MPS-II patients with beneficial results. ${ }^{22}$

Interestingly, one patient (ID 71), was a newborn and had relatively little inflammation. Nevertheless, receptor activator of nuclear factor-KB ligand (RANKL), an important cytokine in bone homeostasis, already showed a clear increase in concentration at birth compared to age-matched controls. Patients that were sampled at the age of 2 months did already show a pro-inflammatory profile. It would be interesting to evaluate whether other MPS newborns also show little inflammation at birth. PPS treatment at diagnosis in patients detected via newborn screening programs might be able to diminish inflammation-related damage in cartilage and improve outcomes of HCT. Whether already transplanted patients also have a beneficial effect of PPS treatment on skeletal and joint disease remains to be evaluated.

At 10 years' follow-up, the majority of inflammatory markers reached the same levels as age-matched controls, implicating that HCT reduces inflammation in MPS patients. It should be noted though that as this study only provides information about systemic presence of these markers, local inflammation could still be occurring and warrants further investigation. Although systemic inflammatory markers decrease over time after transplantation, bone homeostasis markers, including RANKL, remain elevated. Therefore, increased RANKL secretion induced by pro-inflammatory cytokines is most likely not the only mechanism occurring in MPS patients. 
Bone homeostasis is tightly regulated and is a conjunction of mature osteoblasts creating bone and activated osteoclasts resorbing bone. ${ }^{23}$ Osteoblasts are generated from mesenchymal stem cells (MSCs). MSCs initially differentiate into immature osteoblasts, followed by differentiation into mature osteoblasts. ${ }^{24}$ Co-cultures of patient derived GAGs (MPS-1, MPS-2, MPS-6) with osteogenic MSCs showed an inhibiting effect on the differentiation of immature osteoblasts to mature osteoblasts resulting in decreased mineral production, which was most profound when osteoblasts were cultured with GAGs derived from MPS-6 patients. ${ }^{25}$

During the differentiation of osteoblasts, Wnt signaling is a key pathway. ${ }^{26} \mathrm{Wnt} 3 \mathrm{a}$ (canonical) is mainly responsible for stem cell and osteoblast proliferation, whereas Wnt5a (noncanonical) is responsible for stem cell and osteoblast differentiation and thus mineralization. HS binding sites have been found on most canonical Wnts. ${ }^{27,28}$ When osteoblasts are cultured with MPS-1, -2, and - 6 derived GAGs, an increase in Wnt3a/canonical signaling occurs, and therefore prevention of mineralization. ${ }^{25}$ This might be a cause of the increased levels we observe in AXIN1, as this protein is a key component of the canonical Wnt pathway where it is, in complex with glycogen synthase kinase 3 beta (GSK-3 $\beta$ ) and adenomatous polyposis coli (APC), bound to $\beta$-catenin when the canonical Wnt pathway is in an inactivated state. ${ }^{26}$

The most important pathway in bone homeostasis is the RANKL/RANK/OPG pathway. ${ }^{29}$ RANKL, RANK, and OPG are key partners of a system that directly regulates osteoclast differentiation. RANKL, expressed on osteoblasts, binds to RANK present on osteoclast precursors, inducing their differentiation into osteoclasts and stimulating osteoclastogenesis. OPG is a decoy receptor for RANKL that neutralizes RANKL by preventing it to bind to RANK, resulting in decreased osteoclast recruitment. Previous studies have elucidated increased osteoclastogenesis in MPS-1 animals and fibroblasts of MPS-1 patients..$^{30,31}$ MPS-1 osteoblasts produced increased amounts of RANKL and displayed increased amounts of osteoclasts in-vitro. Studies have shown that immature osteoblasts have higher RANKL expression compared to mature osteoblasts, ${ }^{32}$ which could be the reason of higher RANKL expression in MPS patients as GAGs inhibit the differentiation of immature to mature osteoblasts. ${ }^{25}$ Furthermore, GAGs can bind directly to OPG and further increase osteoclastogenesis, ${ }^{33}$ resulting, in combination with decreased mineralization, in the typical skeletal features in MPS patients, named dysostosis multiplex.

The increase in RANK might have treatment implications: Denosumab, a monoclonal human antibody, binds and neutralizes the activity of RANKL and might thus be an interesting drug to treat skeletal disease in MPS patients. Currently, the drug is FDA approved for postmenopausal osteoporosis and for patients with high risk of bone fractures. Recently, the results of a phase III clinical trial on joint destruction in rheumatoid arthritis (RA) patients treated 
with Denosumab have been published. ${ }^{34}$ Denosumab significantly inhibited the progression of joint destruction and led to an increase in bone mineral density but did not affect the joint space narrowing or disease activity scores in RA patients. The effect of Denosumab in MPS has not yet been studied.

Besides increased levels of RANKL, OPG, and AXIN1, increased levels of the growth factors Flt3L and SCF were observed. Increased osteoclastogenesis could be the reason for this increase as osteoclasts are of hematopoietic origin and HSCs give rise to myeloid progenitors upon stimulation with amongst others SCF. ${ }^{35}$ GM-CSF promotes further differentiation of myeloid progenitors in macrophage progenitors. Finally, M-CSF further differentiates cells into the monocyte/macrophage lineage which are considered osteoclasts precursors. Our data show M-CSF (CSF.1) was indeed increased in MPS patients before HCT. After 10 years of follow-up, M-CSF was still statistically different between controls and patients although no longer with a fold change $>2.8$. Flt3L, on the other hand, did continue to differ between patients and controls. Studies have shown Flt3L can be a substitute for M-CSF ${ }^{36}$ and might, in MPS patients, contribute to the differentiation of osteoclasts in a non-CSF dependent matter.

This study has several limitations. First, our controls were phenylketonuria patients and therefore not 'real' healthy controls. However, PKU patients are on a strict diet and showed no symptoms of disease. As it is difficult to collect DBS from healthy children of all ages, this was chosen to be the best 'healthy' control available. The observation that all patients could be separated from controls and the controls showed no inflammation profiles indicates that these second-best controls were sufficient. The second limitation was that only crosssectional data was available for the control group. As a consequence, we could not include control data in the mixed model analyses. We did observe an age-related increase in a substantial number of markers and therefore longitudinal data of controls could increase the power of advanced statistical methods in the future. Finally, as MPS are rare disorders we were unfortunately unable to validate our current findings in a new cohort of patients. Future research on biomarkers, inflammation, and the effect of new therapies on amongst others skeletal disease in MPS should take RANKL, OPG, AXIN1, Flt3L, and SCF into account in order to validate our results. Finally, most biomarker research has been done in plasma or serum samples. DBS, however, are easy to collect (i.e. sampling can be done from home and sent to the laboratory by mail) and showed higher stability of markers over time compared to plasma samples ${ }^{37}$ However, markers found in DBS samples can differ from plasma markers. In contrast to plasma, also markers that are present intracellularly or on the surface of cells are measured when using DBS. Studies comparing biomarkers should therefore take the origin of the sample into account. 
In conclusion, we are the first to demonstrate the long-term effect of HCT on systemic inflammation in MPS patients. Pro-inflammatory markers were elevated pre-HCT and remaind elevated early after HCT. However, the majority have declined to (near-)normal levels after 10 years of follow-up. Nevertheless, markers associated with bone homeostasis continue to be increased despite HCT and most likely reflect the ongoing skeletal disease after transplantation. Future research should evaluate whether these markers could serve as easy accessible biomarkers for the effect of new therapies on musculoskeletal symptoms in MPS patients. Furthermore, anti-inflammatory and anti-RANKL agents might improve the clinical outcomes of MPS patients, especially regarding the musculoskeletal manifestations.

\section{ACKNOWLEDGEMENTS}

All authors would like to thank all parents and patients for participating in this study. The authors would like to thank Prof. dr. M.J.C. Eijkemans and Dr. R. Stellato for their statistical input. 


\section{REFERENCES}

1. Muenzer J. Overview of the mucopolysaccharidoses. Rheumatology. 2011;50(suppl 5):v4-v12.

2. Aldenhoven M, Jones SA, Bonney D, et al. Hematopoietic Cell Transplantation for Mucopolysaccharidosis Patients Is Safe and Effective: Results after Implementation of International Guidelines. Biol Blood Marrow Transplant. 2015;21(6):1106-1109.

3. Boelens JJ, Aldenhoven M, Purtill D, et al. Outcomes of transplantation using various hematopoietic cell sources in children with Hurler syndrome after myeloablative conditioning. Blood. 2013;121(19).

4. Aldenhoven M, Wynn RF, Orchard PJ, et al. Long-term outcome of Hurler syndrome patients after hematopoietic cell transplantation: an international multicenter study. Blood. 2015;125(13):2164-2172.

5. van den Broek BTA, van Egmond-Ebbeling MB, Achterberg JA, et al. Longitudinal analysis of ocular disease in children with Mucopolysaccharidosis I after hematopoietic cell transplantation. Biol Blood Marrow Transplant. November 2019.

6. Langereis EJ, den Os MM, Breen C, et al. Progression of Hip Dysplasia in Mucopolysaccharidosis Type I Hurler After Successful Hematopoietic Stem Cell Transplantation. J Bone Joint Surg Am. 2016;98(5):386-395.

7. Javed A, Aslam T, Jones SA, et al. The effect of haemopoietic stem cell transplantation on the ocular phenotype in mucopolysaccharidosis type I (Hurler). Acta Ophthalmol. 2017:1-5.

8. Simonaro CM, Haskins ME, Schuchman EH. Articular chondrocytes from animals with a dermatan sulfate storage disease undergo a high rate of apoptosis and release nitric oxide and inflammatory cytokines: a possible mechanism underlying degenerative joint disease in the mucopolysaccharidoses. Lab Invest. 2001;81(9):1319-1328.

9. Villani GRD, Gargiulo N, Faraonio R, Castaldo S, Gonzalez Y Reyero E, Di Natale P. Cytokines, neurotrophins, and oxidative stress in brain disease from mucopolysaccharidosis IIIB. J Neurosci Res. 2007;85(3):612-622.

10. Opoka-Winiarska V, Jurecka A, Emeryk A, Tylki-Szymanska A. Osteoimmunology in mucopolysaccharidoses type I, II, VI and VII. Immunological regulation of the osteoarticular system in the course of metabolic inflammation. Osteoarthr Cartil. 2013;21(12):1813-1823.

11. Polgreen LE, Vehe RK, Rudser K, et al. Elevated TNF-alpha is associated with pain and physical disability in mucopolysaccharidosis types I, II, and VI. Mol Genet Metab. 2016;117(4):427-430. 
12. Assarsson E, Lundberg M, Holmquist G, et al. Homogenous 96-plex PEA immunoassay exhibiting high sensitivity, specificity, and excellent scalability. PLoS One. 2014;9(4).

13. Lundberg M, Eriksson A, Tran B, Assarsson E, Fredriksson S. Homogeneous antibody-based proximity extension assays provide sensitive and specific detection of low-abundant proteins in human blood. Nucleic Acids Res. 2011;39(15):e102-e102.

14. Leek JT, Johnson WE, Parker HS, Jaffe AE, Storey JD. The sva package for removing batch effects and other unwanted variation in high-throughput experiments. Bioinformatics. 2012;28(6):882-883.

15. Johnson WE, Li C, Rabinovic A. Adjusting batch effects in microarray expression data using empirical Bayes methods. Biostatistics. 2007;8(1):118-127.

16. Raymond G V, Pasquali M, Polgreen LE, et al. Elevated cerebral spinal fluid biomarkers in children with mucopolysaccharidosis I-H. Sci Rep. 2016;6:38305.

17. Simonaro CM, D’Angelo M, He X, et al. Mechanism of glycosaminoglycanmediated bone and joint disease: implications for the mucopolysaccharidoses and other connective tissue diseases. Am J Pathol. 2008;172(1):112-122.

18. Simonaro CM, Ge Y, Eliyahu E, He X, Jepsen KJ, Schuchman EH. Involvement of the Toll-like receptor 4 pathway and use of TNF-alpha antagonists for treatment of the mucopolysaccharidoses. Proc Natl Acad Sci U S A. 2010;107(1):222-227.

19. Polgreen LE, Kunin-Batson A, Rudser K, et al. Pilot study of the safety and effect of adalimumab on pain, physical function, and musculoskeletal disease in mucopolysaccharidosis types I and II. Mol Genet Metab reports. 2017;10:75-80.

20. Schuchman EH, Ge Y, Lai A, et al. Pentosan polysulfate: a novel therapy for the mucopolysaccharidoses. PLoS One. 2013;8(1):e54459.

21. Hennermann JB, Gökce S, Solyom A, Mengel E, Schuchman EH, Simonaro CM. Treatment with pentosan polysulphate in patients with MPS I: results from an open label, randomized, monocentric phase II study. J Inherit Metab Dis. 2016;39(6):831-837.

22. Orii K, Lim A, Tomatsu S, et al. Safety study of sodium pentosan polysulfate for adult patients with mucopolysaccharidosis Type II. Diagnostics. 2019;9(4):1-18.

23. Evans $\mathrm{CH}$. John Hunter and the origins of modern orthopaedic research. J Orthop Res. 2007;25(4):556-560.

24. Komori T. Regulation of Proliferation, Differentiation and Functions of Osteoblasts by Runx2. Int J Mol Sci. 2019;20(7).

25. Rout-Pitt NB. The effects of undegraded glycosaminoglycans from Mucopolysaccharidoses on osteoblast differentiation and mineralisation In vitro. Thesis -- Univ Adelaide, Sch Med 2015. 2015. 
26. Baron R, Kneissel M. WNT signaling in bone homeostasis and disease: from human mutations to treatments. Nat Med. 2013;19(2):179-192.

27. Binari RC, Staveley BE, Johnson WA, Godavarti R, Sasisekharan R, Manoukian AS. Genetic evidence that heparin-like glycosaminoglycans are involved in wingless signaling. Development. 1997;124(13):2623-2632.

28. Ling L, Dombrowski C, Foong KM, et al. Synergism between Wnt3a and heparin enhances osteogenesis via a phosphoinositide 3-kinase/Akt/RUNX2 pathway. $J$ Biol Chem. 2010;285(34):26233-26244.

29. Boyle WJ, Simonet WS, Lacey DL. Osteoclast differentiation and activation. Nature. 2003;423(6937):337-342.

30. Simonaro CM, D'Angelo M, He X, et al. Mechanism of glycosaminoglycanmediated bone and joint disease: Implications for the mucopolysaccharidoses and other connective tissue diseases. Am J Pathol. 2008;172(1):112-122.

31. Gatto F, Redaelli D, Salvade A, et al. Hurler disease bone marrow stromal cells exhibit altered ability to support osteoclast formation. Stem Cells Dev. 2012;21(9):14661477.

32. Atkins GJ, Kostakis P, Pan B, et al. RANKL expression is related to the differentiation state of human osteoblasts. J Bone Miner Res. 2003;18(6):1088-1098.

33. Théoleyre S, Kwan Tat S, Vusio P, et al. Characterization of osteoprotegerin binding to glycosaminoglycans by surface plasmon resonance: Role in the interactions with receptor activator of nuclear factor $\kappa \mathrm{B}$ ligand (RANKL) and RANK. Biochem Biophys Res Commun. 2006;347(2):460-467.

34. Takeuchi T, Tanaka Y, Soen S, et al. Effects of the anti-RANKL antibody denosumab on joint structural damage in patients with rheumatoid arthritis treated with conventional synthetic disease-modifying antirheumatic drugs (DESIRABLE study): A randomised, double-blind, placebo-controlled phase. Ann Rheum Dis. 2019;78(7):899-907.

35. Ash P, Loutit JF, Townsend KM. Osteoclasts derived from haematopoietic stem cells. Nature. 1980;283(5748):669-670.

36. Lean JM, Fuller K, Chambers TJ. FLT3 ligand can substitute for macrophage colony-stimulating factor in support of osteoclast differentiation and function. Blood. 2001;98(9):2707-2713.

37. Björkesten J, Enroth S, Shen Q, et al. Stability of Proteins in Dried Blood Spot Biobanks. Mol Cell Proteomics. 2017;16(7):1286-1296. 


\section{SUPPLEMENTAL FIGURES}

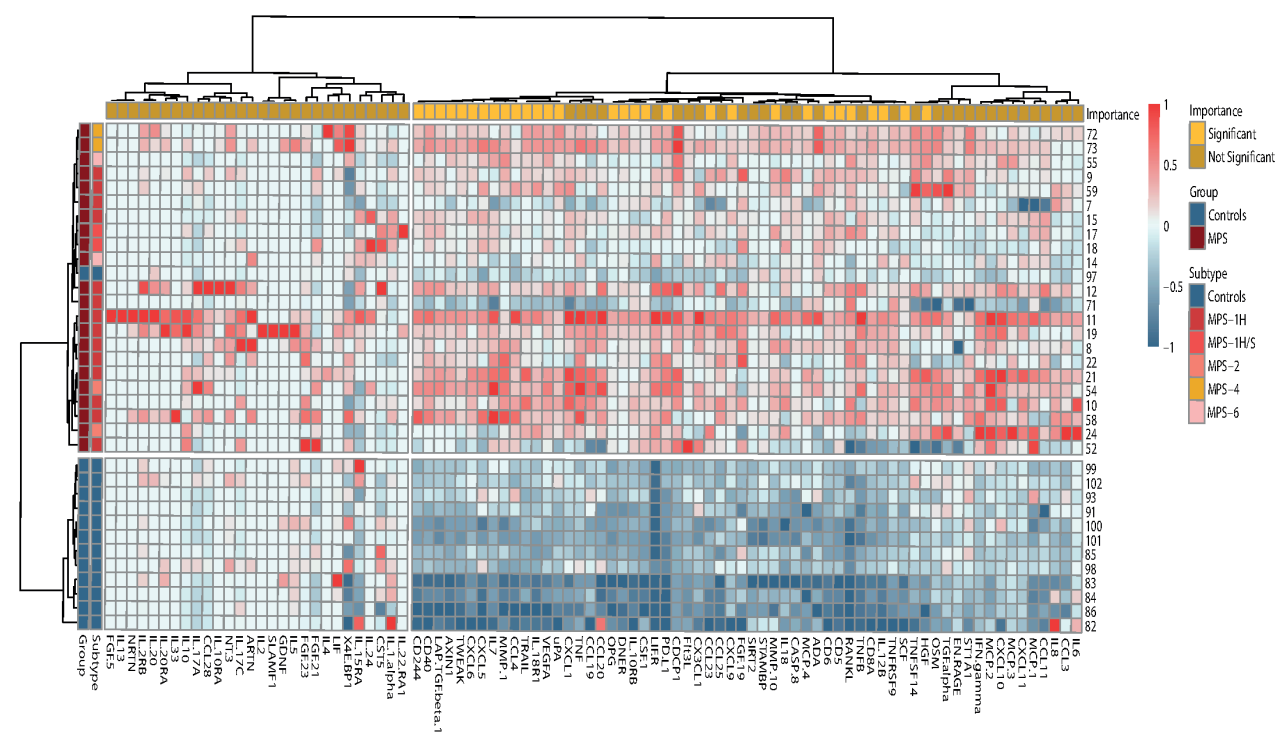

Suppl. figure 1. Heatmap of all inflammation markers in pre-HCT MPS patients $(\mathrm{n}=22)$ and age-matched controls $(n=12)$. 
A
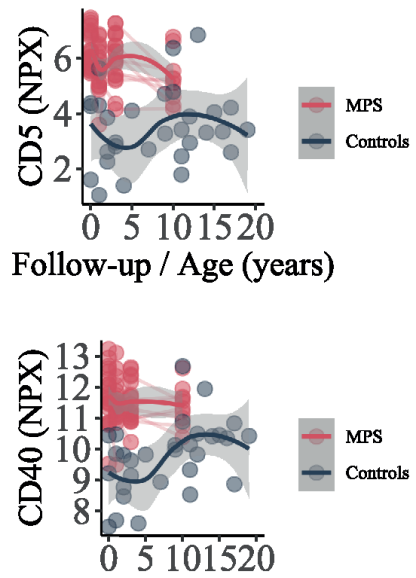

Follow-up / Age (years)

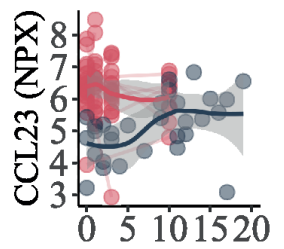

Follow-up / Age (years)

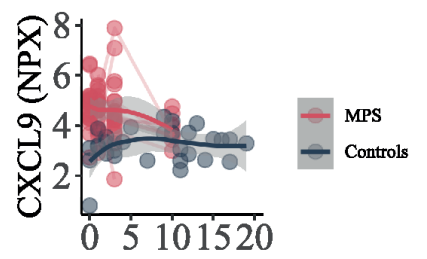

Follow-up / Age (years)

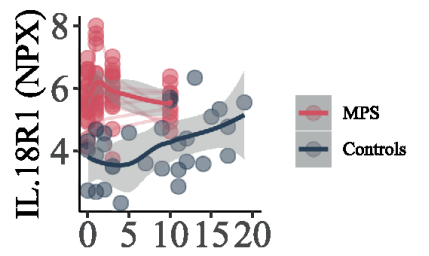

Follow-up / Age (years)
Pro-inflammatory

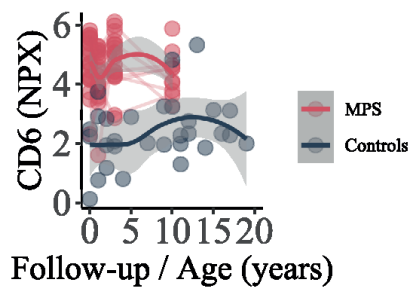

Follow-up / Age (years)

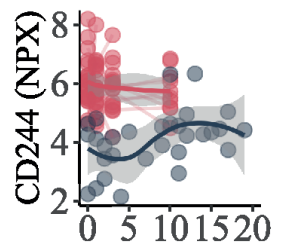

Follow-up / Age (years)
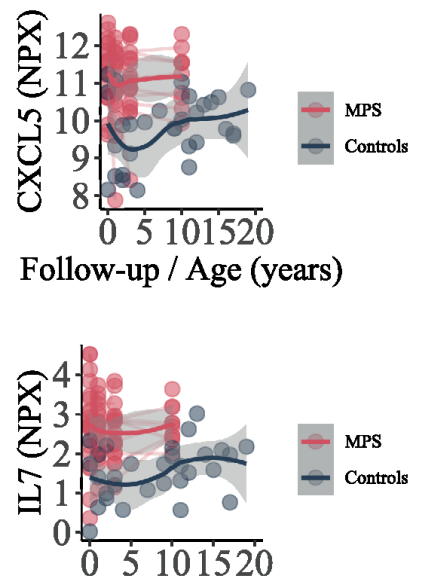

Follow-up / Age (years)

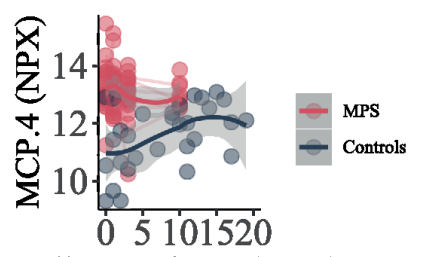

Follow-up / Age (years)

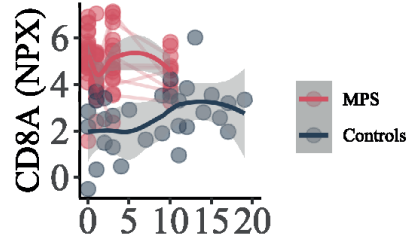

Follow-up / Age (years)

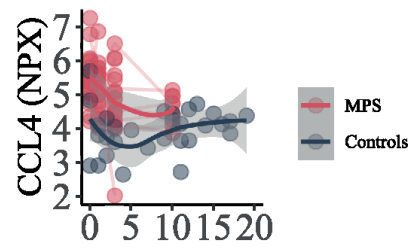

Follow-up / Age (years)

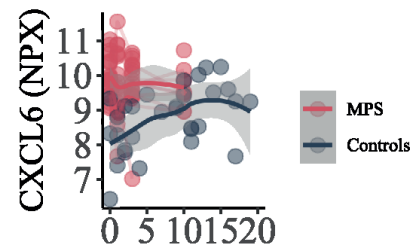

Follow-up / Age (years)

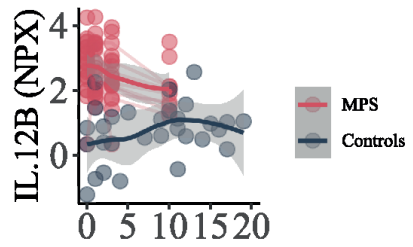

Follow-up / Age (years)

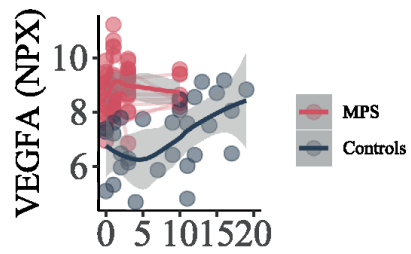

Follow-up / Age (years)

Suppl. figure 2A-C. Pre-HCT important markers for the different pathways: Pro-inflammatory (A), Antiinflammatory (B) and (C) ECM degradation, bone homeostasis, and Apoptosis. Patient data are shown in red, cross-sectional data of controls in blue. Average line is shown with a 'Loess'. X-axes show Follow-up (in years) for patients and Age (in years) for controls. Median age at transplantation was 1.3 years (range $0.2-4.8$ years). 
B

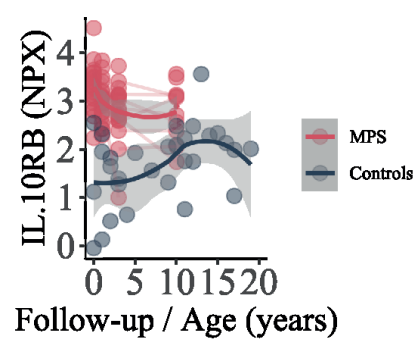

Anti-inflammatory

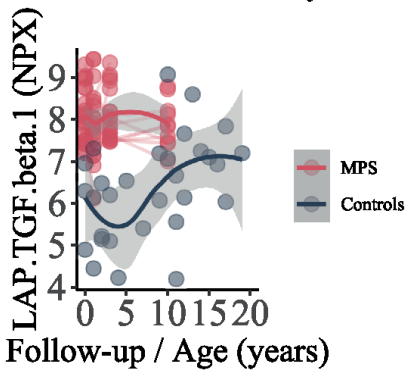

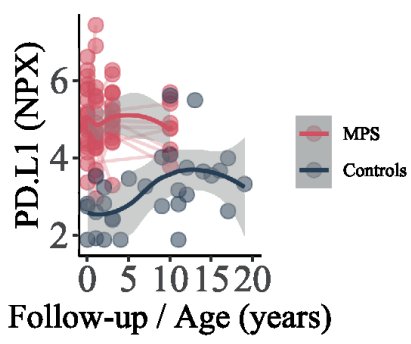

Suppl. figure 2A-C. Pre-HCT important markers for the different pathways: Pro-inflammatory (A), Antiinflammatory (B) and (C) ECM degradation, bone homeostasis, and Apoptosis. Patient data are shown in red, cross-sectional data of controls in blue. Average line is shown with a 'Loess'. X-axes show Follow-up (in years) for patients and Age (in years) for controls. Median age at transplantation was 1.3 years (range $0.2-4.8$ years). 
C
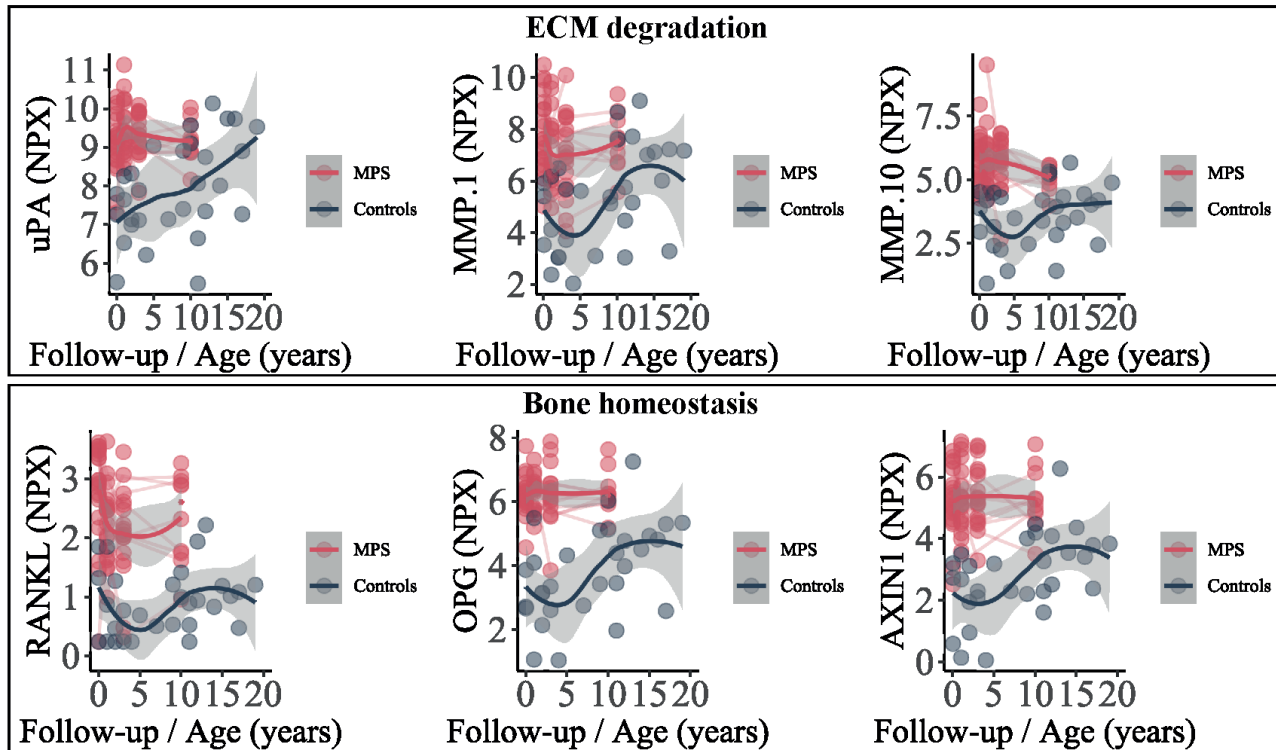

Bone homeostasis

Follow-up / Age (years)
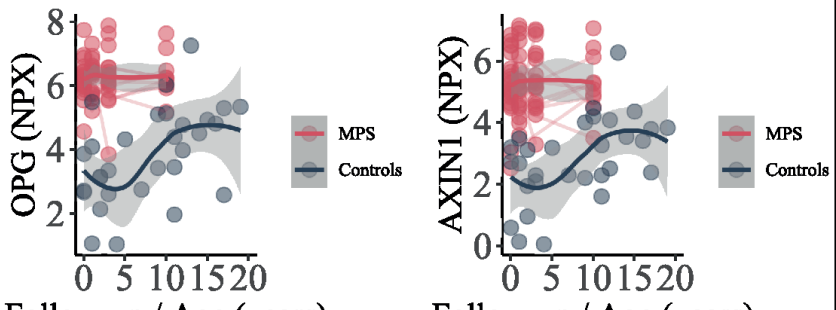

Follow-up / Age (years)

Follow-up / Age (years)
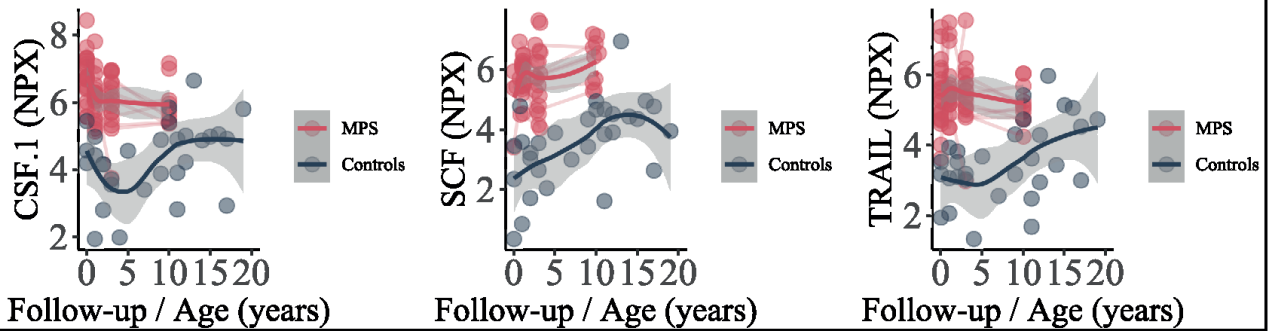

Follow-up / Age (years)

Follow-up / Age (years)

Follow-up / Age (years)
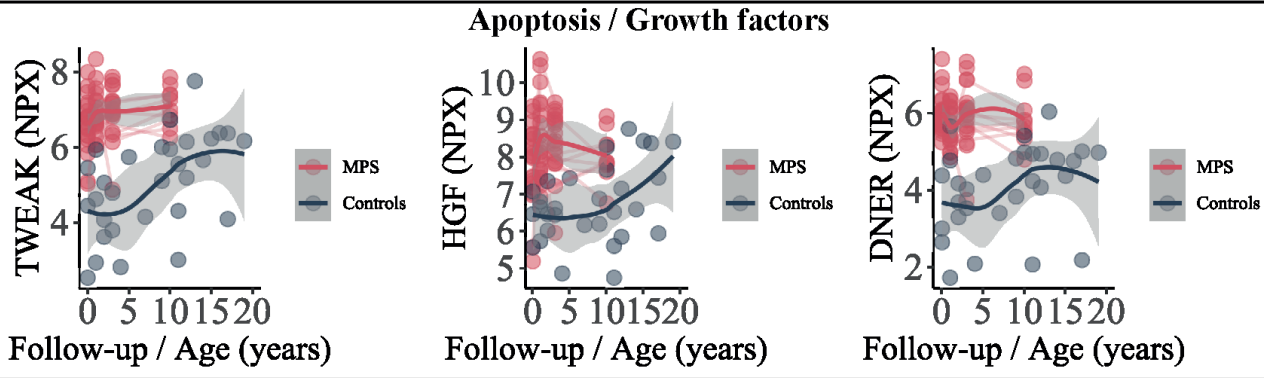

Suppl. figure 2A-C. Pre-HCT important markers for the different pathways: Pro-inflammatory (A), Antiinflammatory (B) and (C) ECM degradation, bone homeostasis, and Apoptosis. Patient data are shown in red, cross-sectional data of controls in blue. Average line is shown with a 'Loess'. X-axes show Follow-up (in years) for patients and Age (in years) for controls. Median age at transplantation was 1.3 years (range $0.2-4.8$ years). 


\section{SUPPLEMENTAL TABLES}

Suppl. table 1. Markers included in the OLINK Inflammation I panel

\begin{tabular}{|l|l|l|l|l|}
\hline ADA & 4E-BP1 & IL-22RA1 & OPG & AXIN1 \\
\hline ARTN & FGF21 & IL-24 & PD-L1 & Flt3L \\
\hline Beta-NGF & FGF-5 & IL-33 & EN-RAGE & FGF23 \\
\hline CCL19 & CX3CL1 & IL-4 & SLAMF1 & FGF-19 \\
\hline CCL20 & GDNF & IL-5 & SIRT2 & \\
\hline CCL23 & HGF & IL-6 & STAMBP & \\
\hline CCL25 & IFN-gamma & IL-7 & SCF & \\
\hline CCL28 & IL-1 alpha & IL-8 & ST1A1 & \\
\hline CCL3 & IL10 & LAPTGF-beta-1 & CD6 & \\
\hline CCL4 & IL-10RA & LIF & CD5 & \\
\hline CXCL1 & IL-10B & LIF-R & CD8A & \\
\hline CXCL10 & IL-12B & CSF-1 & TSLP & \\
\hline CXCL11 & IL-13 & MMP-1 & TNFB & \\
\hline CXCL5 & IL-15RA & MMP-10 & RANKL & \\
\hline CXCL6 & IL-17A & MCP-1 & TRAIL & \\
\hline CXCL9 & IL-17C & MCP-2 & TGF-alpha & \\
\hline CASP-8 & IL-18 & MCP-3 & TWEAK & \\
\hline CD40 & IL-18R1 & MCP-4 & TNF & \\
\hline CDCP1 & IL-2 & CD244 & TNFSF14 & \\
\hline CST5 & IL-2RB & NT-3 & TNFRSF9 & \\
\hline DNER & IL-20 & NRTN & uPA & \\
\hline CCL11 & IL-20RA & OSM & VEGF-A & \\
\hline
\end{tabular}




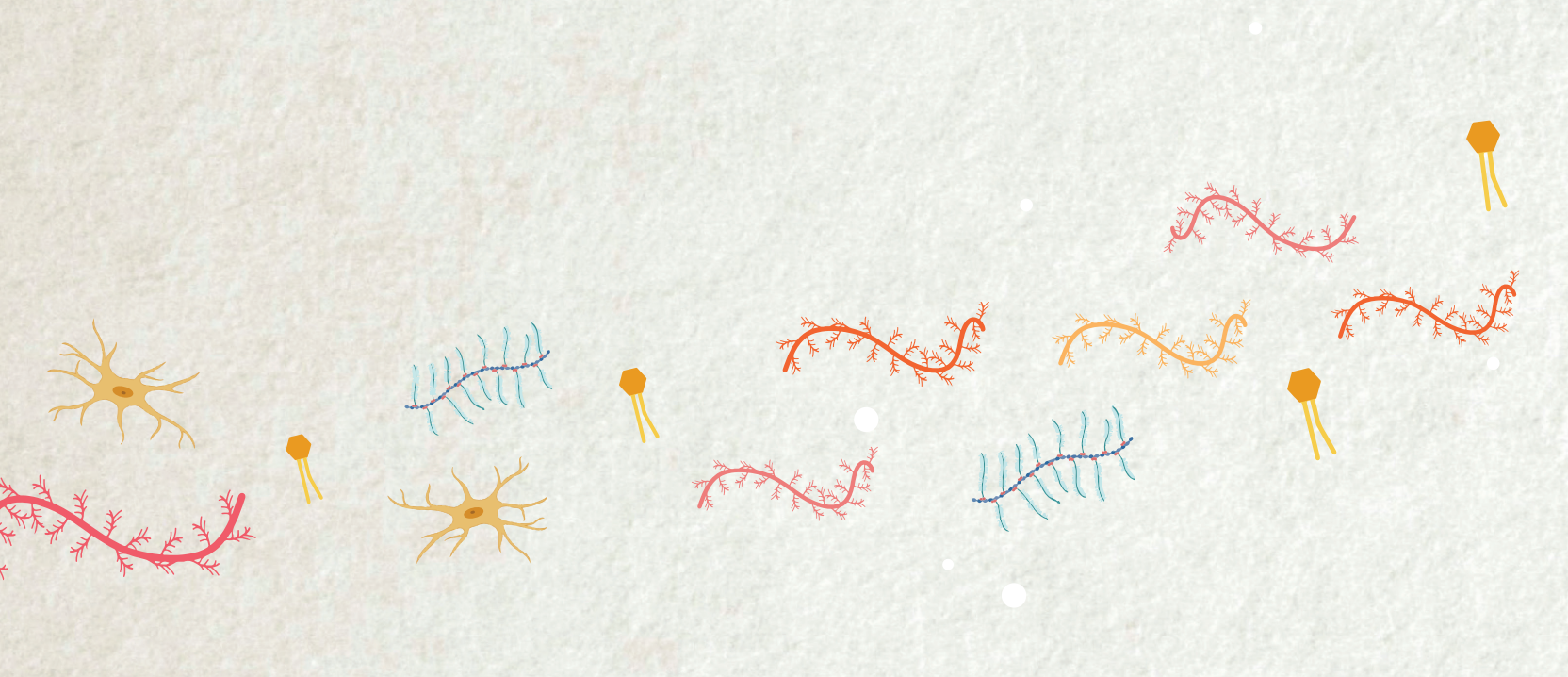



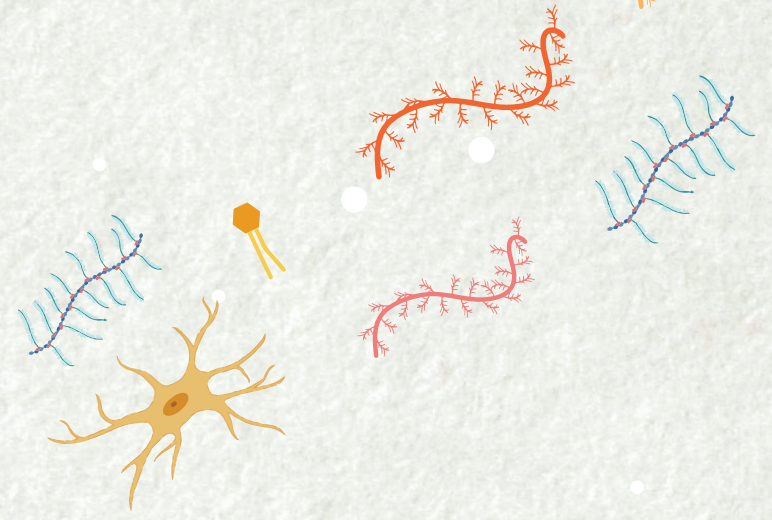

\title{
CHAPTER 9
}

\author{
Hurdles in Treating Hurler's Disease: \\ Potential Routes to Achieve a 'Real' Cure
}

Brigitte T.A. van den Broek

Jaap van Doorn

Charlotte V. Hegeman

Stefan Nierkens

Caroline A. Lindemans

Nanda M. Verhoeven-Duif

Jaap Jan Boelens

Peter M. van Hasselt 


\begin{abstract}
Mucopolysaccharidoses (MPS) are multi-organ devastating diseases for which hematopoietic cell transplantation (HCT) has dramatically increased the overall survival rate. However, despite identification of clinical predictors and harmonized transplantation protocols, disease progression still poses a significant burden to patients, although at a slower pace. To design better therapies, we need to understand why and where current therapies fail. In this review, we discuss important aspects of the underlying disease and the disease progression. We note that the majority of progressive symptoms that occur in "hard-to-treat" tissues are actually tissues that are hard to reach, such as avascular connective tissue or tissues isolated from the circulation by a specific barrier (e.g. blood-brain-barrier, blood-retina-barrier). While easily reached tissues are effectively cured by HCT, disease progression is observed in these "hard-to-reach" tissues. We used these insights to critically appraise ongoing experimental endeavors with regard to their potential to overcome the encountered hurdles and improve long-term clinical outcomes in MPS patients treated with HCT.
\end{abstract}




\section{INTRODUCTION}

It has been more than 50 years since Fratantoni et al. ${ }^{1}$, in 1968, described that co-cultured fibroblasts of Hurler (MPS-1(H)) and Hunter (MPS-2) patients corrected each other enzymatically leading to a mutual reduction in the intracellular accumulation of GAGs. Hurler and Hunter syndrome are two of the seven types of mucopolysaccharidoses (MPS) in which a deficiency of a specific lysosomal enzyme prevents proper degradation of specific metabolites, clinically resulting in a devastating progressive multi-systemic disease and, if severe, in premature death (table 1). ${ }^{2}$ In 1981, Hobbs et al. ${ }^{3}$ reported the first hematopoietic cell transplantation (HCT) in a 1-year old patient with MPS-1(H) based on the principle of cross-correction. HCT has become the standard of care in MPS-1(H), if timely diagnosed. Intense international collaboration during the last decade has identified predictors for clinical outcomes (i.e. myeloablative conditioning, early timing of transplantation, and enzyme activity level in blood after HCT). This has resulted in harmonized transplantation protocols and 5-year survival rates of $>90 \%$. For MPS-1, enzyme replacement therapy (ERT) was introduced in 2003, followed by ERT for MPS 2, 4, 6, and 7.4-6 Unfortunately, ERT comes with serious limitations: e.g. neutralizing antibodies, lack of blood-brain-barrier passage, and huge costs. The combination of ERT (prior) and HCT has failed to show an advantage over HCT alone. ${ }^{7}$

Despite the greatly improved overall survival, current standard treatments still have their 'weak spots' as they are unable to completely halt the disease in specific tissues. Late outcome studies show significant residual disease burden. ${ }^{711}$ While many researchers have been trying to develop new therapies to improve clinical outcomes, primary outcomes in these studies are often enzyme activity and GAG-concentration in receptive tissues: leukocytes in the circulation, brain, liver, spleen, and lungs. This is unfortunate as we are already capable of treating these tissues. We propose, analogous to Sutton's law, that, in order to evaluate whether new therapies are able to improve quality of life in MPS patients, the focus of the effect of new therapies should be on the "hard-to-treat" tissues and how to improve this effect, because obviously: 'that's where the money is'.

To design better strategies, we first need to understand why current therapies fail. Why are some tissues hard to treat? Better understanding of disease pathogenesis and the mechanism of current treatments are therefore necessary. In this review we summarize important aspects of the underlying disease, establish which tissues are "hard-to-treat" and define their unifying characteristics. Furthermore, we critically appraise experimental therapeutic endeavors with regard to their potential to overcome these hurdles and improve long-term clinical outcomes of MPS patients. 
Table 1. Enzyme deficiencies, storage material and clinical manifestations of the different MPS types

\begin{tabular}{|c|c|c|c|c|}
\hline Type & Eponyme & Enzyme deficiency & $\begin{array}{l}\text { Deposited GAGs } \\
\text { (secondary) }\end{array}$ & $\begin{array}{c}\text { Clinical manifestations } \\
\text { before HCT }\end{array}$ \\
\hline I & $\begin{array}{l}\text { Hurler } \\
\text { Hurler/Scheie } \\
\text { Scheie }\end{array}$ & $\alpha$-L-iduronidase & $\begin{array}{l}\text { DS } \\
\text { HS } \\
(\mathrm{KS})\end{array}$ & $\begin{array}{l}\text { Coarse facies, short stature, } \\
\text { dysostosis multiplex, joint } \\
\text { stiffness, spinal cord compression, } \\
\text { organomegaly, corneal clouding, } \\
\text { retinal degeneration, hearing loss, } \\
\text { cardiac/respiratory disease, mental } \\
\text { retardation in severe phenotype }\end{array}$ \\
\hline II & Hunter & Iduronate-2-sulphatase & $\begin{array}{l}\text { DS } \\
\text { HS } \\
(\mathrm{KS})\end{array}$ & $\begin{array}{l}\text { Coarse facies, short stature, } \\
\text { dysostosis multiplex, joint } \\
\text { stiffness, spinal cord compression, } \\
\text { organomegaly, corneal clouding, } \\
\text { retinal degeneration, cardiac/ } \\
\text { respiratory disease, mental } \\
\text { retardation }\end{array}$ \\
\hline III-A & Sanfilippo A & Heparan N-sulphatase & & $\begin{array}{l}\text { Severe mental impairment, } \\
\text { aggressive behaviour, sleep }\end{array}$ \\
\hline III-B & Sanfilippo B & $\alpha-\mathrm{N}$-acetylglucosaminidase & HS & disturbances and dementia, \\
\hline III-C & Sanfilippo $C$ & $\begin{array}{l}\text { Acetyl-coa- } \alpha \text {-glucosaminide } \\
\text { Acetyltransferase }\end{array}$ & $(\mathrm{KS})$ & $\begin{array}{l}\text { relatively mild somatic symptoms, } \\
\text { corneal clouding, respiratory }\end{array}$ \\
\hline III-D & Sanfilippo D & $\begin{array}{l}\mathrm{N} \text {-acetylglucosamine 6- } \\
\text { sulphatase }\end{array}$ & & disease, hearing loss \\
\hline IV-A & Morquio type $A$ & Galactosamine-6-sulphatase & $\begin{array}{l}\mathrm{KS} \\
\mathrm{C} 6 \mathrm{~S}\end{array}$ & $\begin{array}{l}\text { Short stature, ligamentous laxity, } \\
\text { joint hypermobility, dysostosis }\end{array}$ \\
\hline IV-B & Morquio type $B$ & $\beta$-galactosidase & $\mathrm{KS}$ & $\begin{array}{l}\text { multiplex, corneal clouding, } \\
\text { hearing loss, cardiac disease }\end{array}$ \\
\hline VI & Maroteaux-Lamy & $\begin{array}{l}\mathrm{N} \text {-acetylgalactosamine } \\
\text { 4-sulphatase }\end{array}$ & $\begin{array}{l}\mathrm{DS} \\
\mathrm{C} 4 \mathrm{~S} \\
(\mathrm{KS})\end{array}$ & $\begin{array}{l}\text { Coarse facies, short stature, } \\
\text { dysostosis multiplex, joint } \\
\text { stiffness, organomegaly, corneal } \\
\text { clouding and cardiac/respiratory } \\
\text { disease; no mental impairment }\end{array}$ \\
\hline VII & Sly & $\beta$-glucuronidase & $\begin{array}{l}\text { HS } \\
\text { DS } \\
\text { C4S } \\
\text { C6S } \\
(\mathrm{KS})\end{array}$ & $\begin{array}{l}\text { Coarse facies, short stature, } \\
\text { dysostosis multiplex, joint } \\
\text { stiffness, spinal cord compression, } \\
\text { organomegaly, cardiac disease, } \\
\text { corneal clouding and mild mental } \\
\text { impairment }\end{array}$ \\
\hline IX & Natowicz & Hyaluronidase & HA & $\begin{array}{l}\text { Short stature, polyarthropathy, } \\
\text { periarticular soft tissue masses } \\
\text { with painful swelling and } \\
\text { acetabular erosion }\end{array}$ \\
\hline
\end{tabular}

DS, dermatan sulfate; HS, heparin sulfate; KS, keratin sulfate; C6S, chondroitin-6-sulfate; C4S, chondroitin-4-sulfate 


\section{UNDERSTANDING THE UNDERLYING DISEASE}

In healthy individuals, glycosaminoglycans (GAGs), formerly called mucopolysaccharides, represent complex sugar molecules and are degraded in a stepwise manner by enzymes in the lysosome. ${ }^{12}$ The two main groups are sulphated GAGs (heparan sulphate; HS, dermatan sulphate; DS, keratan sulphate; KS, and chondroitin sulphate; CS), and non-sulphated GAGs (hyaluronic acid; HA). All GAG chains, except HA, are linked to a core protein to form proteoglycans (PG). ${ }^{12}$ PGs are important components of the ground substance in the extracellular matrix (ECM) of connective tissue. Furthermore, they contribute in the ordering process of collagen fibres. ${ }^{13}$ During development, PGs are important in the assembly of ECM in tissue morphogenesis to determine form and function of a tissue. ${ }^{14}$

ECM is essential in the communication between cells. Communication is modulated via interactions between GAG chains and ECM components such as collagen, fibronectin, and laminin. ${ }^{13,15}$ Furthermore, some PGs are known to bind and regulate a number of distinct proteins, including chemokines, cytokines, and growth factors. ${ }^{16}$ Finally, to allow diffusion of nutrients and waste products, the interstitial fluid of connective tissue contains a large amount of water. ${ }^{17,18}$

Hence, GAGs, that may also occur as constituent of PGs are important components of connective tissue which is found amongst others in the skeleton, cornea, cartilage, tendons, and to a lesser extent in the brain (figure 1). ${ }^{19}$ Both free GAGs and proteoglycans are biologically active molecules and are involved in critical cellular pathways. They can sequester extracellular humoral factors, modulate the function of cell surface receptors in signal transduction and cross talk between cells. PGs are expressed during skeletal development and they are spatially and temporally regulated in a highly defined pattern. ${ }^{20}$

In MPS, a deficiency of a lysosomal enzyme results in the accumulation of partially degraded GAGs. The primary GAG storage leads to perturbation of cellular, tissue and organ homeostasis. ${ }^{21}$ Subsequently, the pathways in which they are involved will become affected. These secondary effects, such as impaired autophagy, increased apoptosis, and inflammation are also important effectors of the disease symptoms (figure 2 ). ${ }^{22}$ The main clinical symptoms are coarse facial features, hepato- and splenomegaly, dysostosis multiplex, joint stiffness, recurrent respiratory infections, sleep apnea, heart disease, and, in severe subtypes, cognitive impairment and a reduced life expectancy. The time of onset, rate of progression and extensiveness of the disease manifestations vary depending on the type of MPS. ${ }^{2}$ Details on the specific types of MPS can be found in table 1. 


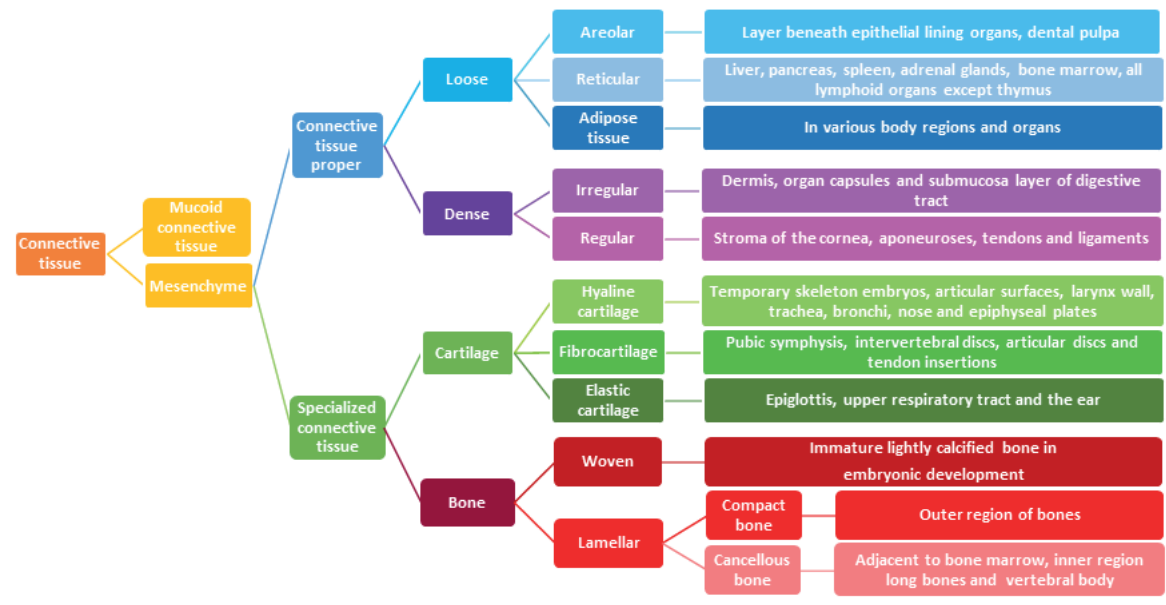

Figure 1. Classification of connective tissue

UNDERSTANDING DISEASE PROGRESSION IN "HARD-TO-TREAT" TISSUES; in search of common themes.

\section{Disease progression related to connective tissue}

Skeletal malformations

Skeletal deformities are extremely common in MPS disorders and the beneficial impact of HCT is limited. ${ }^{23-25}$ Thoracolumbar kyphosis, hip dysplasia and genu valgum are progressive in the majority of patients, albeit at a slower pace. ${ }^{26-28}$ Several factors are thought to contribute to skeletal malformations. Firstly, ossification and mineralization depend on the distribution of growth factors (i.e. IGFs, FGF, PTHrP, Indian Hedgehog) and GAGs play a major role in the regulation of these signaling pathways. ${ }^{29,30}$ Secondly, bone remodeling and growth are based on the ossification of cartilage which is disturbed due to the malfunction of chondrocytes containing accumulated GAGs and altered cathepsin K activity (figure 2). ${ }^{31}$ This is supported by the presence of non-ossified cartilaginous precursors in bones of MPS patients. ${ }^{32,33}$ Cartilage is avascular and thus depends on enzyme delivery from the circulation via diffusion. The distance that enzymes have to overcome in bone and articular cartilage is 
rather large and therefore ossification continues to be impaired. Thirdly, GAG accumulation stimulates secondary pathophysiological processes in ECM, like inflammation, contributing to osteopenia and the abnormal shape of bones seen in MPS patients. ${ }^{34}$ Finally, abnormal weight-bearing forces influence the morphology of bone structures. ${ }^{35,36}$ Malformations in these structures already present before HCT alter the forces between them and contribute to further progression of hip dysplasia. Progression of hip deformations also influences the progression of genu valgum. ${ }^{37,38}$ Importantly, many of these malformations already start to develop in utero.

In conclusion, many bone defects have already developed at the time of HCT due to abnormal ossification, lack of bone remodeling, inflammation and abnormal forces. Post-HCT, enzyme availability remains insufficient in the avascular, diffusion-dependent, cartilage. Ongoing alterations of growth factor signaling pathways and inflammation may play additional roles in the alterations of chondrogenesis and bone growth. ${ }^{39}$

Joints, tendons, and ligaments

In all types of MPS, except MPS-4, GAG accumulation in ligaments, tendons, and joint capsules results in joint contractures. ${ }^{40-42}$ Studies on joint stiffness post-HCT show conflicting outcomes. ${ }^{26,43-46}$ The joint capsule is a dense connective tissue layer that enhances joint security, bone placement, and may also extend into the joint to form a fibrocartilaginous articular disc or meniscus. Normally, the capsule contains blood vessels, but altered forces can drive this tissue into a more fibrocartilage type that becomes much less vascularized. In this process, an increase in collagen type II, KS, DS, and CS is seen. ${ }^{47}$ As a consequence, changes in fibril arrangement, and spacing and size occur, which is destructive for tendons and ligaments, since a proper fibril arrangement is pivotal to create functional structures. Besides joint contractions, weakening of the tendons is also seen which partly explains the thoracolumbar kyphosis. ${ }^{48}$

Furthermore, carpal tunnel syndrome (CTS), caused by median nerve constriction in the wrist due to GAG deposition in the transverse carpal ligament, occurs in $89 \%$ of patients despite HCT. Also, trigger digits, caused by GAG deposition in the flexor tendons, are frequently observed, despite treatment. ${ }^{26,49-52}$

Thus, GAGs accumulate in ligaments, tendons, and joint capsules despite HCT. These tissues have in common that they are avascular connective tissues. Hence, they rely on diffusion of enzyme from the circulation after HCT. Moreover, most of these structures consist of dense connective tissue in which diffusion of nutrients is even more difficult and the arrangement of the structure is highly important. This likely explains the poor efficacy of current systemic treatments. 


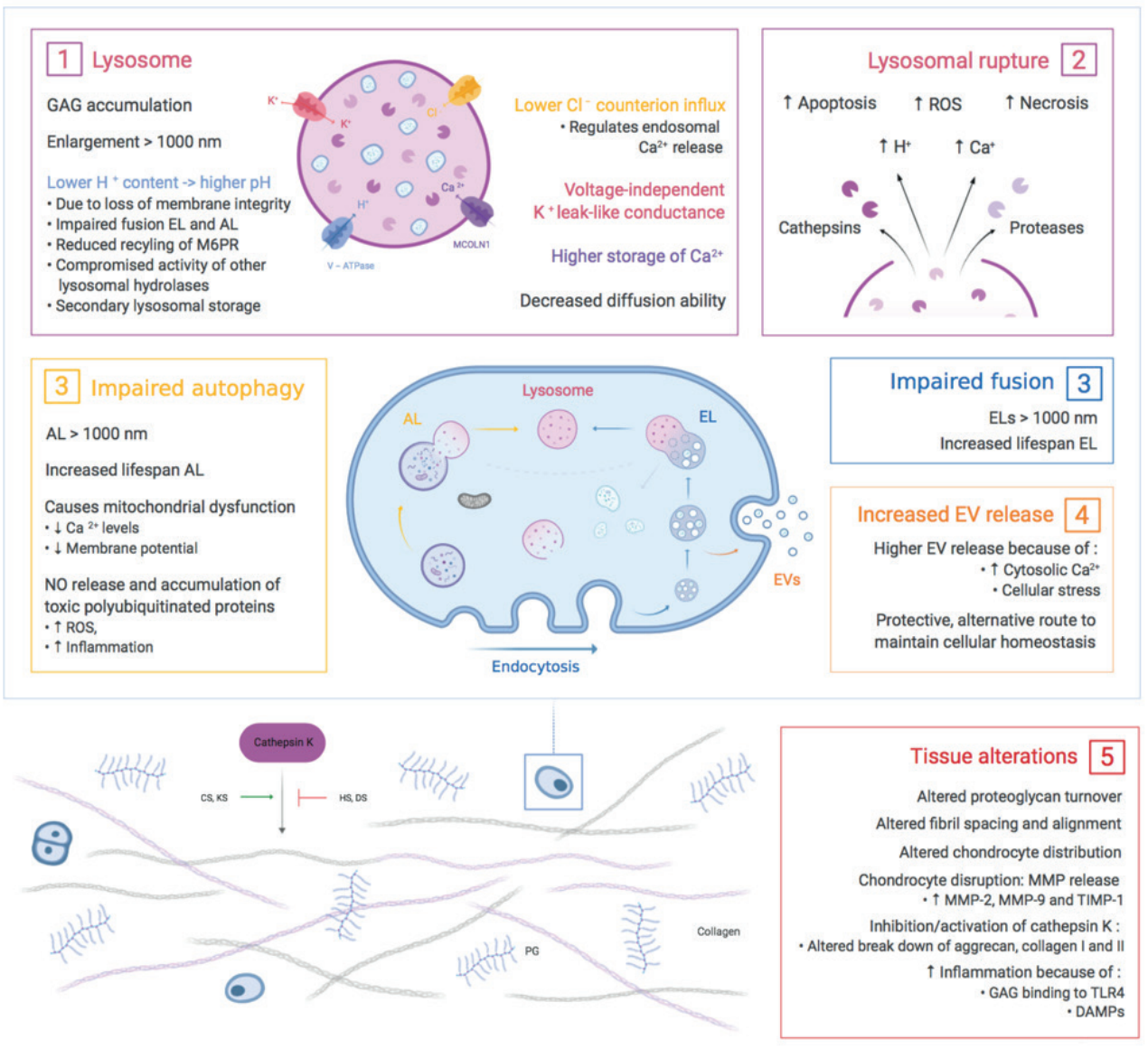

Figure 2. Effects of accumulated lysosomal products on cellular and tissue homeostasis. 1) Accumulation of GAGs in the lysosome leads to enlargement of the lysosome followed by a loss of membrane integrity. Leakage of $\mathrm{H}^{+}$out of the lysosome results in a higher $\mathrm{pH}$ and may lead to compromised activity of lysosomal hydrolases and secondary lysosomal storage. Furthermore, the higher $\mathrm{pH}$ results in impaired fusion of endolysosomes and autolysosomes and a reduced recycling of the M6P-receptor. Finally enlarged lysosomes have decreased diffusion ability inside the cell. 2) Enlargement of the lysosomal ultimately results in lysosomal rupture with the escape of cathepsins and proteases in the cell. Simultaneously, lysosomal rupture leads to increased concentration of $\mathrm{H}^{+}$ and $\mathrm{Ca}^{2+}$ in the cytosol. Together this results in increased ROS production, necrosis and apoptosis of cells. 3) The dysfunctional lysosome leads to impaired autophagy and fusion with endolysosomes. Therefore, an increased lifespan of autolysosomes and endolysosomes is seen. Impaired autophagy subsequently results in mitochondrial dysfunction. 4) The increased concentration of cytosolic $\mathrm{Ca}^{2+}$ and cellular stress stimulates the release of extracellular vesicles. This is an alternative, protective route of the cell to maintain cellular homeostasis. 5) The accumulation of GAGs in the cell and the secondary affected mechanisms result in tissue alterations and destruction. Connective tissue is the most affected tissue in MPS patients. Studies show altered proteoglycan turnover, altered fibril spacing and alignment, altered chondrocyte distribution and disruption leading to increased concentrations of MMP-2, MMP-9 and TIMP-1. Undegradaded GAGs lead, depending on the type of GAG, to inhibition or activation of cathepsin $\mathrm{K}$ which is necessary for the degradation of aggrecan, collagen I and II. Finally, HS and cellular stress lead to inflammation via activation of the TLR4 pathway. Created with Biorender.com. 


\section{Corneal clouding}

Progression of corneal clouding is observed post HCT. ${ }^{53-55}$ The cornea, a transparent avascular connective tissue, consists of epithelial and endothelial cells, keratocytes, collagen and PGs. ${ }^{56}$ GAG accumulation affects the size of keratocytes and disrupts the regular network of the stroma, resulting in abnormal arrangement, spacing, and size of collagen fibrils. ${ }^{57,58}$ Tear fluid could potentially be the supplier of donor-enzyme after HCT. However, enzyme activity in tear fluid of MPS-1 patients after HCT remains low despite normalization in the circulation, insinuating poor enzyme penetration. ${ }^{55}$

\section{Heart valves}

While disease manifestations in the coronary arteries and the heart muscle are effectively addressed following HCT, the mitral and aortic valve deformities, such as thickening of the valves, persist. In some, this may lead to progressive valvular dysfunction. ${ }^{59,60}$ The heart valves contains a highly specialized and organized ECM in which the alignment of collagen fibers is extremely important. ${ }^{61}$ GAG accumulation leads to abnormal fibril organization and weakening of valvular connective tissue, due to a decrease in stiffness and an increase in extensibility which is associated with valvular prolapse. ${ }^{62-65}$ Once again, disease progression is seen in an avascular connective tissue and enzyme unavailability is most likely the limiting factor.

\section{Disease progression unrelated to connective tissue}

\section{Retinal degeneration}

Despite successful HCT, retinal degeneration worsenes. ${ }^{53}$ In contrast to the avascular cornea, the retina is vascularized but enzyme availability is hampered by the blood-retina-barrier (BRB), which likely explains progression of retinal degeneration. Kaneko et al. ${ }^{66}$ used enhanced green fluorescent protein-positive bone marrow cells in mice to study whether donor cells end up in the retina after HCT. They compared damaged retinas (induced by injection of N-methyl-N-nitrosourea or creation of retinal detachment) with normal retinas and showed only a minute number of donor cells end up in uninjured retinas of mice up to 12 months following HCT. Damaged retinas however, showed massive recruitment of donor-derived cells into the retina. ${ }^{66}$ Whether retinas of MPS patients show similar damage and thus recruit donor-derived macrophages after HCT to produce enzyme locally warrants further investigation.

Taken together, we propose "hard-to-treat" tissues in MPS are actually "hard-to-reach" tissues where two major hurdles need to be taken, namely overcoming avascular zones and blood-tissue barriers. Poorly vascularized tissues are completely dependent on diffusion of the enzyme from the circulation which can pose a significant hurdle if the distance is too 
large or when the tissue is very dense. An advantage of HCT is its ability to allow monocytes to migrate to the brain and thus pass the blood-brain-barrier, suggesting this physiological barrier is overcome. MPS-3 patients, however, in which CNS symptoms predominate, deteriorate despite $\mathrm{HCT}^{67}$ Speculatively, this may be explained by the predominant accumulating GAG in MPS-3 being HS. HS is most important in the brain, and the CNS of these patients may require an amount of enzyme that, apparently, is not sufficiently provided by HCT. Furthermore, progression of retinal degeneration insinuates that effect of therapy is still inhibited by the BRB. Therefore, we propose that additional local therapies or therapies that enhance transport of systemic available enzyme to connective tissues or tissues isolated from the circulation due to a specific barrier could help to improve long term outcomes in MPS patients.

\section{TOWARDS TREATING "HARD-TO-REACH” TISSUES}

Based on the general considerations above, alternative approaches for enzyme delivery to "hard-to-reach" tissues have been proposed. These include, ordered on their proximity to clinical use, gene therapy, direct local administration, small molecule therapies, targeted enzyme delivery systems, mesenchymal stem cell therapy, and focused ultrasound therapy.

\section{Gene therapy}

Gene therapy (GT) is the most successful experimental therapy in the last decade. Viral GT has been studied widely, however, non-viral strategies have been explored as well.

\section{Viral gene therapies}

Viral gene therapy in MPS has been explored for retroviruses, lentiviruses (LV) and adenoassociated viruses (AAV). ${ }^{68}$ Due to the risk of mutagenesis when using retroviruses, research now focusses mainly on LVs and AAVs. Ex-vivo LV gene therapy is used in combination with autologous stem cell therapy, aiming for supra-physiological enzyme activity levels in the circulation with the rationale to improve penetration in poor responding tissues. Autologous gene-modified HCT is currently being studied in a clinical trial for MPS-1 (clinicaltrials.gov, NCT03488394) and MPS-3A (clinicaltrials.gov, NCT04201405) and is being developed for MPS-3B. An important advantage of autologous stem cell gene therapy is reduced morbidity and mortality due to the absence of graft failure and graft-versus-host-disease. ${ }^{69}$ Patients could, however, develop antibodies against the enzyme which could subsequently reduce the effect of therapy. While the initial results are promising, it is still an open question whether higher hematological available enzyme is able to overcome the diffusion distances in poorly responding avascular tissues and completely cure MPS. Besides gene-modified autologous 
HCT, direct administration of GT is also being studied and will be addressed later.

\section{Non-viral gene therapies}

A non-viral gene therapy strategy are the Sleeping Beauty (SB) transposons. ${ }^{70}$ SB transposons consist of a system using two plasmids: one containing the internal sequence for the system, the inverted terminal repeat (ITR) and the gene of interest, and the other one containing the SB transposase that recognizes the ITR to ensure translocation of the gene into the genome. The SB strategy was studied in MPS-1 mice and showed efficacy in most somatic organs but not all (i.a. the kidney, heart and aorta). Also the central nervous system was not treated with the use of SB transposons. Therefore, the SB strategy is not yet sufficient for treatment of MPS. ${ }^{71}$

\section{Direct local administration}

To overcome both avascularity of tissues or blood-tissue barriers, direct local administration of recombinant enzyme has been studied intra-articular in animal models for MPS-1 and MPS-6. ${ }^{72-74}$ By injecting the recombinant enzyme directly into the joint cavity of MPS-6 cats, obvious histological improvements were consistently detectable. Following treatment, clearance of lysosomal storage in synovial cells and chondrocytes was seen. Joints which received recombinant enzyme had improved thickness of cartilage, fewer cartilage lesions, better overall joint shape, healthy well-vascularized subchondral bone, and improved synovium compared to the contralateral buffer-treated joints. These encouraging results have led to the treatment of two MPS-6 patients with monthly intra-articular injections in the hips for a period of 2 years. ${ }^{73}$ The results, however, have yet to be published. Wang et al. ${ }^{74}$ found similar results for intra-articular recombinant enzyme in MPS-1 dogs. Administration into intra-articular spaces resulted in reduction of GAG storage not only in the synovium and chondrocytes in the articular surface of the joint cartilage, but also in chondrocytes further away from the articular surface.

Major disadvantages of injecting recombinant enzyme are the need for repeated injections and the potential risk of antibody formation. Vance et al. ${ }^{75}$ explored AAV-mediated gene therapy for enzyme delivery by intrastromal injection as an effective treatment of MPS-1-related corneal clouding. Seven days after injection of an AAV8G9-optimized enzyme construct in human corneas, the enzyme was over-produced in the corneal stroma with widespread distribution in multiple cell types and demonstrated a 10-fold elevation of enzyme activity without any indications of toxicity. ${ }^{75}$ Furthermore, AAV-mediated gene therapy directly administered into the brain has been proven to be successful in animals. ${ }^{76-78}$ This has resulted in clinical trials which are currently open for MPS-1 (clinicaltrials.gov, NCT03580083), MPS-2 (clinicaltrials. gov, NCT03566043), and MPS-3A (clinicaltrials.gov, NCT03612869 and NCT02716246). 


\section{Small molecule therapies}

Alternative strategies that overcome the transport obstacles are strategies that improve enzyme activity locally or decrease the workload of the faulty enzyme.

A significant part (circa 70\%) of severe MPS phenotypes are caused by premature stopcodon mutations. ${ }^{79}$ Therefore, stop-codon read-through therapy (SCRT) could be a promising approach. However, most of the well-known drugs with read-through effects are aminoglycosides, like gentamicin and chloramphenicol, and are toxic. Therefore, a less toxic derivate was constructed, named NB84. The results of a phase I clinical trial with the synthetic version of NB84, ELX-02, have recently been published and showed an acceptable safety profile in healthy adults. ${ }^{80}$ Clinical efficacy in MPS patients however, has yet to be proven. A nonaminoglycoside SCRT compound is PTC-124. Although for PTC-124 promising results were seen in MPS mouse models, an open-label clinical phase II trial was ended early because of low enrolment (EudraCT number, 2014-002596-28). No conclusions of the effect could be drawn.

\section{Substrate reduction or optimization therapy}

Molecules which reduce the synthesis of GAGs, such as genistein and rhodamine B, are a subject of research in MPS-1, -2 , and $-3 .{ }^{81-83}$ A phase III study of genistein in MPS-3 patients was completed in 2018, however the results have not yet been published (EudraCT, 2013-001479-18). An earlier pilot study showed genistein was safe, reduced urine GAG concentration and arrested behavioural and cognitive deficits. ${ }^{84}$ In MPS-2 patients, genistein improved joint and connective tissue elasticity. ${ }^{85}$ Besides substrate reduction therapies, substrate optimization therapy (SOT) has also been applied. SOT includes compounds that alter the accumulating substrate to make it more amenable for degradation by other enzymes. In-vitro studies showed that modified GAGs were indeed more susceptible for degradation in fibroblasts and the therapy is currently being developed for MPS- $1 .{ }^{86,87}$

\section{Chaperone therapy}

Chaperones are small proteins that aid correct folding of enzymes leading to an increase of residual enzyme activity. ${ }^{88}$ In-vitro experiments on several MPS 3B- and 3C-causing mutations indicated that pharmaceutical chaperones can bind and stabilize mutant enzymes, and improve their enzymatic activity. Thus, the use of pharmaceutical chaperones to alter enzyme activity might be plausible, especially for those mutations that cause abnormal glycosylation patterns. ${ }^{88}$

Small molecule therapies show promising in-vitro results. However, their in-vivo efficacy has yet to be proven in clinical trials. As most compounds are $<50 \mathrm{kDa}$, they are able to cross the BBB and possibly the BRB. Although not suitable as general standard therapies, they could be helpful in achieving better clinical outcomes in specific mutations seen in MPS patients. 


\section{Targeted enzyme delivery systems}

Targeted drug delivery seeks to concentrate the medication in the tissues of interest and can potentially improve drug delivery in both avascular tissues or isolated tissues due to a bloodtissue barrier. Two types of targeted enzyme delivery systems have been studied in recent years: a so-called Trojan horse strategy and a nano-targeted delivery route.

\section{Trojan horse strategy}

The Trojan horse strategy entails the use of fusion proteins to cross a therapeutic over a specific barrier. ${ }^{89}$ The therapeutic part of the fusion protein can be either conjugated to a monoclonal antibody (Mab) against a tissue-specific target-receptor or to an endogenous ligand for natural binding to the receptor. ${ }^{90}$ In MPS, fusion proteins have mainly been studied to cross the $\mathrm{BBB}$ via the receptors of transferrin, insulin, LDL, or lectin and demonstrated to create higher yields of enzyme in the brain. ${ }^{91-95}$ Targeted delivery of drugs to bones has been investigated for several bone diseases with the use of a hydroxyapatite-binding site, or alendronate (a drug with a high affinity for bone) and show promising results in-vitro. ${ }^{96}$ In-vivo efficacy has yet to be proven. This approach has not been studied for MPS so far but could be a strategy to get the deficient enzyme into the cartilage and bone and possibly achieve a better clinical response.

\section{Nano-targeted delivery}

Nano-therapy has been proposed for both gene products and direct enzyme delivery, and offers promising therapeutic methods in lysosomal storage diseases. ${ }^{97,98}$ It uses different strategies, including micelles, liposomes, nano-emulsions or nanoparticles. The choice for which biomaterial is used depends on the biochemicals of the desired drug. ${ }^{97}$ Nano-targeted delivery of enzyme might be specifically interesting for the treatment of bone and cartilage disease in MPS patients. Wang et al. ${ }^{99}$ created a liposome specific for hydroxyapatite, which binds with a high affinity to collagen-hydroxyapatite compositions in-vitro. This suggests the possibility to use mineral-targeted ligands in nano-particles for the delivery of lysosomal enzymes to mineralized tissues. Bidone et al. ${ }^{100}$ studied the use of intra-articular administration of cationic nano-emulsions complexed with a plasmid encoding for the IDUA protein. They demonstrated the localization of the nano-emulsion in synovial joints, cellular uptake by synoviocytes, high cell viability, and a 10-fold increase in IDUA-expression in the joint $48 \mathrm{~h}$ after injection. IDUA-activity showed a 5-fold increase in synovial fluid and a 20 fold increase in synovial joints. ${ }^{100}$

\section{Mesenchymal stem cell therapy and extracellular vesicles}

Bone marrow, in addition to HSCs, contains mesenchymal stem cells (MSCs). MSCs are multi-potent stromal cells that are characterized by their intrinsic self-renewal capacity 
and ability to differentiate into cells of the mesenchymal lineage, including osteoblasts, chondrocytes. ${ }^{101,102}$ They are important in tissue homeostasis and regeneration due to their immunomodulatory potential and release of trophic factors. ${ }^{103}$ Direct infusion of expanded MSCs however, leads to entrapment in the lungs and fast clearance by the spleen. ${ }^{104}$ Furthermore, several studies have shown MSC's do not engraft after infusion and the effect is thus only temporary. ${ }^{104}$ Recently however, studies indicate that extracellular vesicles (EVs) are mainly responsible for the positive effects of MSCs. ${ }^{105} \mathrm{EVs}$ are small endosome-derived lipid nanoparticles $(40-500 \mathrm{~nm})$ and play an important role in intercellular trafficking. ${ }^{106}$ Due to their very small size they are interesting for the delivery of enzyme to avascular or bloodtissue barrier isolated tissues. EVs can carry macromolecules, including enzymes, to adjacent cells. ${ }^{107}$ While literature on EVs in metabolic diseases is scarce, EVs are known to contain lysosomal proteins. ${ }^{108}$ Furthermore, transplantation of MSCs in the cornea of MPS-3 and MPS-7 mice showed that cross-correction of the deficient cells was indeed mediated by EVs and corrected GAG accumulation. ${ }^{108,109}$ EVs from MSCs could thus be a potential deliverer of enzyme to deficient cells.

\section{Focused ultrasound therapy}

In the past years, ultrasound contrast agents have been used in diagnostic ultrasound imaging. ${ }^{110}$ They can also carry drugs or genes to select targeted tissues and cells for treatment or transfection. ${ }^{111}$ The mechanism behind (focused) ultrasound therapy (FUS) relies on the administration of microbubbles. These microbubbles are lipid or protein containing shells filled with a heavy gas. The use of ultrasonic pressure waves on microbubbles causes them to collapse, resulting in a local widening of endothelial tight junctions, called sonoporation. ${ }^{12}$ Sonoporation causes temporary disruption of, for example, the BBB. ${ }^{113}$ As a consequence, larger molecules up to $2000 \mathrm{kDa}$ are able to pass the BBB. ${ }^{114}$ The disturbance in the BBB leads to an increased drug concentration in the brain as already demonstrated in several disease models, including the delivery of rhIDUA in the case of MPS- 1 mice. ${ }^{115}$

Ultrasound therapy has also been investigated to deliver drugs past the blood-retinabarrier. Using a rat model, Park et al. ${ }^{116}$ demonstrated that burst ultrasound together with a microbubble agent was able to induce transient increases of retinal vascular permeability for retinal drug delivery in rats without any significant side effects. The BRB appeared to be restored within a few hours, which provided a suitable time-window for ocular pharmaceutical agent delivery while avoiding undesired effects that may result from long-term BRB disruption. In light of this knowledge, FUS therapy could be especially interesting for cell and gene therapies targeting the brain and retina as it would create a one-time temporary disruption of the $\mathrm{BBB}$ and $\mathrm{BRB}$ which allows the product to enter the tissue of interest and possibly increase bio distribution. 


\section{CONCLUSION}

HCT alone is not sufficient to cure MPS disorders. Therefore, the management of MPS will most likely evolve from monotherapy into combination therapy. A combination of HCT or autologous gene-modified HCT with additional therapies as mentioned above could be the future for successful and complete treatment of MPS (figure 3). With this review we have summarized two important hurdles that explain disease progression in "hard-toreach" tissues in MPS patients: avascularity and tissue-specific barriers, both leading to unavailability of enzyme. The majority of disease progression occurs in connective tissue because of its avascularity. Additionally, specific barriers, like the blood-retina-barrier and the blood-brain-barrier, hamper an optimal effect of current standard therapies. Albeit the best possibility at this time, solely increasing the amount of hematological available enzyme with autologous gene-modified HCT will probably lower residual disease burden, but is, based on these theories, most likely not the holy grail in curing MPS. Based on the nature and characteristics of the different MPS subtypes, each might require a different and unique treatment strategy for successful outcomes. Furthermore, as part of the damage already occurs in utero, newborn screening programs are extremely important to identify patients in an early disease state to prevent further irreversible damage. Finally, biomarkers related to the extent of residual disease or the secondary manifestations such as impaired autophagy, increased apoptosis, and inflammation are necessary to evaluate the effect of (new) therapies on "hard-to-reach" tissues, because in the end: 'that's where the money is'. 
Tissue barriers

Vascularity
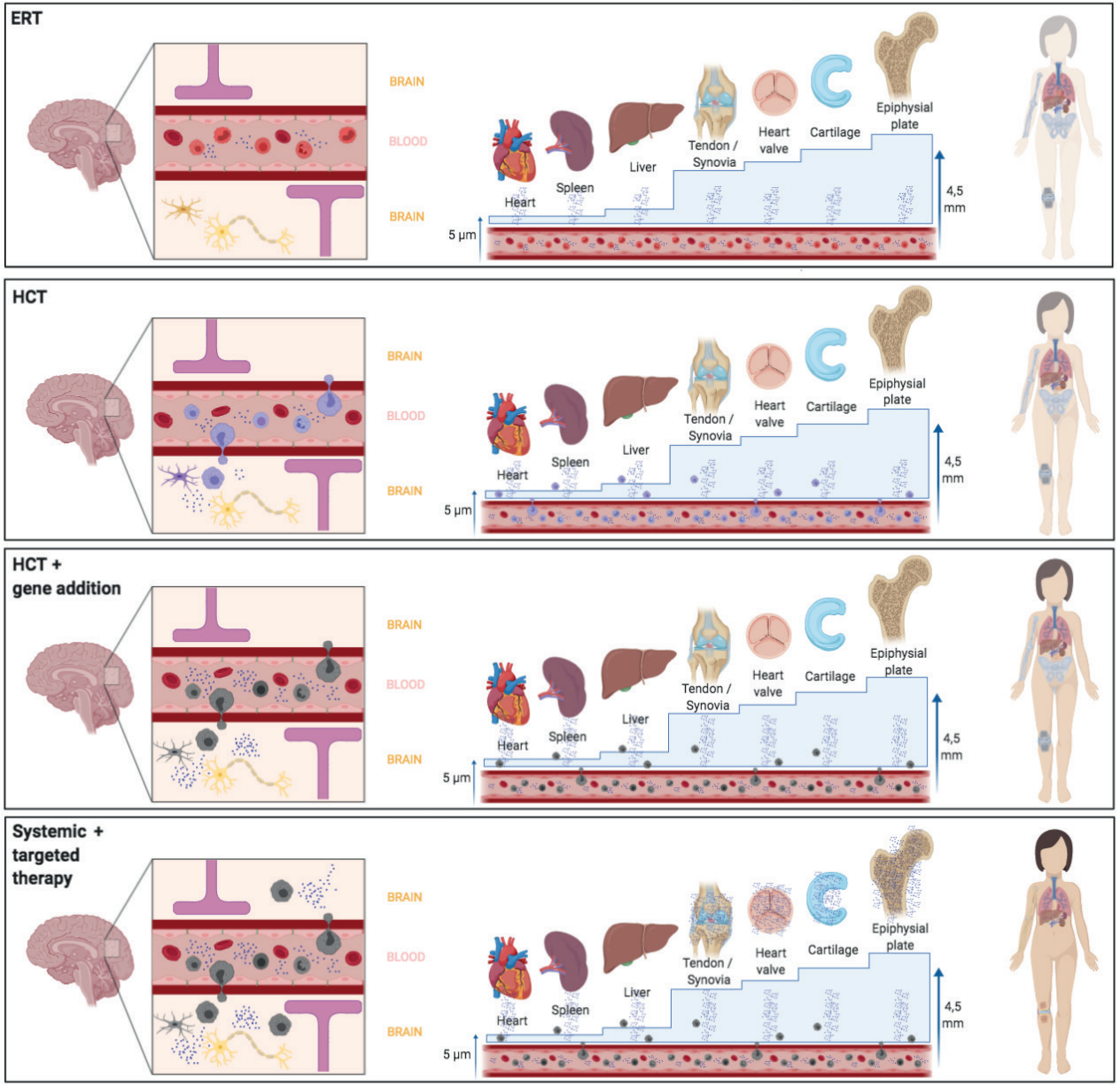

Figure 3. Overview of different therapy strategies on the two major hurdles in MPS. Patients treated with current standard treatments, ERT and HCT, still experience significant disease progression. Two major hurdles in failure of these treatments in specific tissues are the presence of a physical barrier such as the BBB or the BRB and the vascularity of the tissue. Connective tissues, where most disease progression is seen and which are avascular, are isolated from the circulation and thus completely depend on diffusion of monocytes and free enzyme. For the epiphyseal plate for instance, the distance to overcome is $4.5 \mathrm{~mm}$ which is too large and enzyme availability in the epiphyseal plate is therefore still nihil, despite treatment. Solely increasing the available amount of enzyme in the circulation, like in the gene therapy strategies, will most likely not solve this hurdle. Furthermore, it is unknown whether monocytes migrate passed the BRB into the retina like it is assumed in the central nervous system. Progression of retinal degeneration insinuates the available amount of enzyme is at least insufficient. Therefore, the route to cure MPS disease is to focus on increasing enzyme availability in these 'hard-to-reach' tissues. Created with Biorender.com. 


\section{REFERENCES}

1. Fratantoni JC, Hall CW, Neufeld EF. Hurler and Hunter Syndromes: Mutual Correction of the Defect in Cultured Fibroblasts. Science. 1968; 162: 570-572.

2. Muenzer J. Overview of the mucopolysaccharidoses. Rheumatology. 2011; 50: v4-v12.

3. Hobbs JR, Hugh-Jones K, Barrett AJ, et al. Reversal of clinical features of Hurler's disease and biochemical improvement after treatment by bone-marrow transplantation. Lancet (London, England). 1981; 2: 709-712.

4. Concolino D, Deodato F, Parini R. Enzyme replacement therapy: efficacy and limitations. Ital J Pediatr. 2018 ;44: 120.

5. Kuiper G, Nijmeijer SCM, Roelofs MJM, Lee JH, Hollak CEM, Bosch AM. Limited data to evaluate real-world effectiveness of enzyme replacement therapy for mucopolysaccharidosis type I. J Inherit Metab Dis. 2019; 42: 762-775.

6. Valayannopoulos V, Wijburg FA. Therapy for the mucopolysaccharidoses. Rheumatology. 2011; 50: 49-59.

7. Boelens JJ, Aldenhoven M, Purtill D, et al. Outcomes of transplantation using various hematopoietic cell sources in children with Hurler syndrome after myeloablative conditioning. Blood. 2013; 121: 3981-3987.

8. Aldenhoven M, Jones SA, Bonney D, et al. Hematopoietic Cell Transplantation for Mucopolysaccharidosis Patients Is Safe and Effective: Results after Implementation of International Guidelines. Biol Blood Marrow Transplant. 2015; 21: 1106-1109.

9. Wang J, Luan Z, Jiang H, et al. Allogeneic Hematopoietic Stem Cell Transplantation in Thirty-Four Pediatric Cases of Mucopolysaccharidosis-A Ten-Year Report from the China Children Transplant Group. Biol Blood Marrow Transplant. 2016;22:2104-2108.

10. Eisengart JB, Rudser KD, Xue Y, et al. Long-term outcomes of systemic therapies for Hurler syndrome: an international multicenter comparison. Genet Med. 2018;20:1423-1429.

11. Boelens JJ, Rocha V, Aldenhoven M, et al. Risk factor analysis of outcomes after unrelated cord blood transplantation in patients with hurler syndrome. Biol Blood Marrow Transplant. 2009; 15: 618-625.

12. Gandhi NS, Mancera RL. The Structure of Glycosaminoglycans and their Interactions with Proteins. Chem Biol Drug Des. 2008; 72: 455-482.

13. Scott JE. Proteoglycan-fibrillar collagen interactions. Biochem J. 1988;252(2):313-323.

14. Toole BP. Glycosaminoglycans in Morphogenesis. In: Hay ED, ed. Cell Biology of 
Extracellular Matrix. Boston, MA: Springer US; 1981:259-294.

15. Jackson RL, Busch SJ, Cardin AD. Glycosaminoglycans: molecular properties, protein interactions, and role in physiological processes. Physiol Rev. 1991;71(2):481-539.

16. Häcker U, Nybakken K, Perrimon N. Heparan sulphate proteoglycans: the sweet side of development. Nat Rev Mol Cell Biol. 2005;6(7):530-541.

17. Junqueira LC, Mescher AL. Junqueira’s Basic Histology: Text \& Atlas/Anthony L. Mescher. New York [etc.]: McGraw-Hill Medical,; 2013.

18. Ombregt L, Ombregt L. Connective tissue. A Syst Orthop Med. January 2013:29-51.e3.

19. Ruoslahti E. Brain extracellular matrix. Glycobiology. 1996;6(5):489-492.

20. Schwartz NB, Domowicz M. Chondrodysplasias due to proteoglycan defects. Glycobiology. 2002;12(4):57R-68R.

21. Fecarotta S, Gasperini S, Parenti G. New treatments for the mucopolysaccharidoses: from pathophysiology to therapy. Ital J Pediatr. 2018;44(S2):124.

22. Clarke LA. The mucopolysaccharidoses: a success of molecular medicine. Expert Rev Mol Med. 2008;10.

23. Oussoren E, Brands MMMG, Ruijter GJG, der Ploeg AT van, Reuser AJJ. Bone, joint and tooth development in mucopolysaccharidoses: relevance to therapeutic options. Biochim Biophys Acta. 2011;1812(11):1542-1556.

24. van der Linden MH, Kruyt MC, Sakkers RJB, de Koning TJ, Oner FC, Castelein RM. Orthopaedic management of Hurler's disease after hematopoietic stem cell transplantation: a systematic review. J Inherit Metab Dis. 2011;34(3):657-669.

25. Langereis EJ, den Os MM, Breen C, et al. Progression of Hip Dysplasia in Mucopolysaccharidosis Type I Hurler After Successful Hematopoietic Stem Cell Transplantation. J Bone Joint Surg Am. 2016;98(5):386-395.

26. Aldenhoven M, Wynn RF, Orchard PJ, et al. Long-term outcome of Hurler syndrome patients after hematopoietic cell transplantation: an international multicenter study. Blood. 2015;125(13):2164-2172.

27. Miebach E, Church H, Cooper A, et al. The craniocervical junction following successful haematopoietic stem cell transplantation for mucopolysaccharidosis type I H (Hurler syndrome). J Inherit Metab Dis. 2011;34(3):755-761.

28. Taylor M, Khan S, Stapleton M, et al. Hematopoietic stem cell transplantation for mucopolysaccharidoses; past, present, and future. Biol Blood Marrow Transplant. 2019.

29. Gatto F, Redaelli D, Salvade A, et al. Hurler disease bone marrow stromal cells exhibit altered ability to support osteoclast formation. Stem Cells Dev. 
2012;21(9):1466-1477.

30. Habuchi H, Habuchi O, Kimata K. Sulfation pattern in glycosaminoglycan: does it have a code? Glycoconj J. 2004;21(1-2):47-52.

31. Wilson S, Hashamiyan S, Clarke L, et al. Glycosaminoglycan-mediated loss of cathepsin K collagenolytic activity in MPS I contributes to osteoclast and growth plate abnormalities. Am J Pathol. 2009;175(5):2053-2062.

32. Russell C, Hendson G, Jevon G, et al. Murine MPS I: insights into the pathogenesis of Hurler syndrome. Clin Genet. 2008;53(5):349-361.

33. Palmucci S, Attinà G, Lanza ML, et al. Imaging findings of mucopolysaccharidoses: a pictorial review. Insights Imaging. 2013;4(4):443-459.

34. Kingma SDK, Wagemans T, IJlst L, et al. Altered interaction and distribution of glycosaminoglycans and growth factors in mucopolysaccharidosis type I bone disease. Bone. 2016;88:92-100.

35. Fairbank HAT. Congenital dislocation of the hip: with special reference to the anatomy. Br J Surg. 1930;17(67):380-416.

36. Masterson EL, Murphy PG, O'meara A, Moore DP, Dowling FE, Fogarty EE. Hip dysplasia in Hurler's syndrome: orthopaedic management after bone marrow transplantation. J Pediatr Orthop. 1996;16(6):731-733.

37. Guo S, Zhou Y, Yang D, Yang X. Lower-limb valgus deformity associated with developmental hip dysplasia. Chin Med J (Engl). 2012;125(22):3956-3960.

38. Siffert RS. Patterns of deformity of the developing hip. Clin Orthop Relat Res. $1981 ;(160): 14-29$.

39. Opoka-Winiarska V, Jurecka A, Emeryk A, Tylki-Szymanska A. Osteoimmunology in mucopolysaccharidoses type I, II, VI and VII. Immunological regulation of the osteoarticular system in the course of metabolic inflammation. Osteoarthr Cartil. 2013;21(12):1813-1823.

40. Aldenhoven M, Sakkers RJB, Boelens J, de Koning TJ, Wulffraat NM. Musculoskeletal manifestations of lysosomal storage disorders. Ann Rheum Dis. 2009;68(11):1659-1665.

41. Pastores GM, Meere PA. Musculoskeletal complications associated with lysosomal storage disorders: Gaucher disease and Hurler-Scheie syndrome (mucopolysaccharidosis type I). Curr Opin Rheumatol. 2005;17(1):70-78.

42. Morishita K, Petty RE. Musculoskeletal manifestations of mucopolysaccharidoses. Rheumatology. 2011;50(suppl_5):v19-v25.

43. Aldenhoven M, Boelens JJ, de Koning TJ. The clinical outcome of Hurler syndrome after stem cell transplantation. Biol Blood Marrow Transplant. 2008;14(5):485-498.

44. Weisstein JS, Delgado E, Steinbach LS, Hart K, Packman S. Musculoskeletal 
manifestations of Hurler syndrome: long-term follow-up after bone marrow transplantation. J Pediatr Orthop. 2004;24(1):97-101.

45. Souillet G, Guffon N, Maire I, et al. Outcome of 27 patients with Hurler's syndrome transplanted from either related or unrelated haematopoietic stem cell sources. Bone Marrow Transplant. 2003;31(12):1105-1117.

46. Breider MA, Shull RM, Constantopoulos G. Long-term effects of bone marrow transplantation in dogs with mucopolysaccharidosis I. Am J Pathol. 1989;134(3):677.

47. Ralphs JR, Benjamin M. The joint capsule: structure, composition, ageing and disease. J Anat. 1994;184(Pt 3):503.

48. Kuiper G-A, Langereis EJ, Breyer S, et al. Treatment of thoracolumbar kyphosis in patients with mucopolysaccharidosis type I: results of an international consensus procedure. Orphanet J Rare Dis. 2019;14(1):17.

49. Yuen A, Dowling G, Johnstone B, Kornberg A, Coombs C. Carpal tunnel syndrome in children with mucopolysaccaridoses. J Child Neurol. 2007;22(3):260-263.

50. Aslam R, Hendriksz CJ, JesterA. Objective results of median nerve decompression and tenosynovectomy for carpal tunnel syndrome in patients with mucopolysaccharidoses Types I and II. J Hand Surg (European Vol. 2015;40(2):216-218.

51. Van Heest AE, House J, Krivit W, Walker K. Surgical treatment of carpal tunnel syndrome and trigger digits in children with mucopolysaccharide storage disorders. J Hand Surg Am. 1998;23(2):236-243.

52. Viskochil D, Muenzer J, Guffon N, et al. Carpal tunnel syndrome in mucopolysaccharidosis I: a registry-based cohort study. Dev Med Child Neurol. 2017;59(12):1269-1275.

53. Gullingsrud EO, Krivit W, Summers CG. Ocular abnormalities in the mucopolysaccharidoses after bone marrow transplantation: longer follow-up. Ophthalmology. 1998;105(6):1099-1105.

54. Summers CG, Fahnehjelm KT, Pitz S, et al. Systemic therapies for mucopolysaccharidosis: ocular changes following haematopoietic stem cell transplantation or enzyme replacement therapy-a review. Clin Experiment Ophthalmol. 2010;38:34-42.

55. van den Broek BTA, van Egmond-Ebbeling MB, Achterberg JA, et al. Longitudinal analysis of ocular disease in children with Mucopolysaccharidosis I after hematopoietic cell transplantation. Biol Blood Marrow Transplant. November 2019.

56. Sugar J. Corneal manifestations of the systemic mucopolysaccharidoses. Ann Ophthalmol. 1979;11(4):531-535.

57. Alroy J, Haskins M, Birk DE. Altered corneal stromal matrix organization is associated with mucopolysaccharidosis I, III and VI. Exp Eye Res. 1999;68(5):523-530. 
58. Fahnehjelm KT, Ashworth JL, Pitz S, et al. Clinical guidelines for diagnosing and managing ocular manifestations in children with mucopolysaccharidosis. Acta Ophthalmol. 2012;90(7):595-602.

59. Braunlin EA, Stauffer NR, Peters $\mathrm{CH}$, et al. Usefulness of bone marrow transplantation in the Hurler syndrome. Am J Cardiol. 2003;92(7):882-886.

60. Stauffer NR, Braunlin E, Whitley C, Krivit W, Bass J. Echocardiographic follow-up of Hurler syndrome after bone marrow transplantation. Circulation. 1991;84:462a.

61. Hinton RB, Yutzey KE. Heart Valve Structure and Function in Development and Disease. Annu Rev Physiol. 2011;73(1):29-46. doi:10.1146/annurevphysiol-012110-142145

62. Cigliano A, Gandaglia A, Lepedda AJ, et al. Fine structure of glycosaminoglycans from fresh and decellularized porcine cardiac valves and pericardium. Biochem Res Int. 2012.

63. Barber JE, Kasper FK, Ratliff NB, Cosgrove DM, Griffin BP, Vesely I. Mechanical properties of myxomatous mitral valves. J Thorac Cardiovasc Surg. 2001;122(5):955-962.

64. Grande-Allen KJ, Griffin BP, Ratliff NB, Cosgrove DM, Vesely I. Glycosaminoglycan profiles of myxomatous mitral leaflets and chordae parallel the severity of mechanical alterations. J Am Coll Cardiol. 2003;42(2):271-277.

65. Schoen FJ. Evolving concepts of cardiac valve dynamics: the continuum of development, functional structure, pathobiology, and tissue engineering. Circulation. 2008;118(18):1864-1880.

66. Kaneko H, Nishiguchi KM, Nakamura M, Kachi S, Terasaki H. Characteristics of bone marrow-derived microglia in the normal and injured retina. Invest Ophthalmol Vis Sci. 2008;49(9):4162-4168.

67. Welling L, Marchal JP, van Hasselt P, van der Ploeg AT, Wijburg FA, Boelens JJ. Early Umbilical Cord Blood-Derived Stem Cell Transplantation Does Not Prevent Neurological Deterioration in Mucopolysaccharidosis Type III. 2014:63-68.

68. Scarpa M, Orchard PJ, Schulz A, et al. Treatment of brain disease in the mucopolysaccharidoses. Mol Genet Metab. 2017;122S:25-34.

69. Biffi A. Hematopoietic Stem Cell Gene Therapy for Storage Disease: Current and New Indications. Mol Ther. 2017;25(5):1155-1162. doi:10.1016/j.ymthe.2017.03.025

70. Aronovich EL, Hackett PB. Lysosomal storage disease: gene therapy on both sides of the blood-brain barrier. Mol Genet Metab. 2015;114(2):83-93.

71. Aronovich EL, Bell JB, Khan SA, et al. Systemic correction of storage disease in MPS I NOD/SCID mice using the sleeping beauty transposon system. Mol Ther. 2009;17(7):1136-1144. 
72. Auclair D, Hopwood JJ, Lemontt JF, Chen L, Byers S. Long-term intra-articular administration of recombinant human $\mathrm{N}$-acetylgalactosamine-4-sulfatase in feline mucopolysaccharidosis VI. Mol Genet Metab. 2007;91(4):352-361.

73. Auclair D, Ketteridge D, Oates S, Hopwood JJ, Byers S. An overview of intra-articular therapy for mucopolysaccharidosis VI. J Pediatr Rehabil Med. 2010;3(1):3-6.

74. Wang RY, Aminian A, McEntee MF, et al. Intra-articular enzyme replacement therapy with rhIDUA is safe, well-tolerated, and reduces articular GAG storage in the canine model of mucopolysaccharidosis type I. Mol Genet Metab. 2014;112(4):286-293.

75. Vance M, Llanga T, Bennett W, et al. AAV Gene Therapy for MPS1-associated Corneal Blindness. Sci Rep. 2016;6:2-11.

76. Belur LR, Temme A, Podetz-Pedersen KM, et al. Intranasal Adeno-Associated Virus Mediated Gene Delivery and Expression of Human Iduronidase in the Central Nervous System: A Noninvasive and Effective Approach for Prevention of Neurologic Disease in Mucopolysaccharidosis Type I. Hum Gene Ther. 2017;28(7):576-587.

77. Hordeaux J, Hinderer C, Goode T, et al. Toxicology Study of Intra-Cisterna Magna Adeno-Associated Virus 9 Expressing Human Alpha-L-Iduronidase in Rhesus Macaques. Mol Ther Methods Clin Dev. 2018;10:79-88.

78. Hordeaux J, Hinderer C, Buza EL, et al. Safe and Sustained Expression of Human Iduronidase After Intrathecal Administration of Adeno-Associated Virus Serotype 9 in Infant Rhesus Monkeys. Hum Gene Ther. 2019;30(8):957-966.

79. Khan SA, Peracha H, Ballhausen D, et al. Epidemiology of mucopolysaccharidoses. Mol Genet Metab. 2017;121(3):227-240.

80. Leubitz A, Frydman-Marom A, Sharpe N, van Duzer J, Campbell KCM, Vanhoutte F. Safety, Tolerability, and Pharmacokinetics of Single Ascending Doses of ELX-02, a Potential Treatment for Genetic Disorders Caused by Nonsense Mutations, in Healthy Volunteers. Clin Pharmacol drug Dev. 2019.

81. Jakobkiewicz-Banecka J, Piotrowska E, Narajczyk M, Baranska S, Wegrzyn G. Genistein-mediated inhibition of glycosaminoglycan synthesis, which corrects storage in cells of patients suffering from mucopolysaccharidoses, acts by influencing an epidermal growth factor-dependent pathway. J Biomed Sci. 2009;16:26.

82. Roberts ALK, Thomas BJ, Wilkinson AS, Fletcher JM, Byers S. Inhibition of glycosaminoglycan synthesis using rhodamine B in a mouse model of mucopolysaccharidosis type IIIA. Pediatr Res. 2006;60(3):309-314.

83. Derrick-Roberts ALK, Jackson MR, Pyragius CE, Byers S. Substrate Deprivation Therapy to Reduce Glycosaminoglycan Synthesis Improves Aspects of Neurological and Skeletal Pathology in MPS I Mice. Dis (Basel, Switzerland). 2017;5(1). 
84. Piotrowska E, Jakobkiewicz-Banecka J, Maryniak A, et al. Two-year followup of Sanfilippo Disease patients treated with a genistein-rich isoflavone extract: assessment of effects on cognitive functions and general status of patients. Med Sci Monit Int Med J Exp Clin Res. 2011;17(4):CR196.

85. Marucha J, Tylki-Szymanska A, Jakobkiewicz-Banecka J, et al. Improvement in the range of joint motion in seven patients with mucopolysaccharidosis type II during experimental gene expression-targeted isoflavone therapy (GET IT). Am J Med Genet A. 2011;155A(9):2257-2262.

86. Kirkegaard T. Emerging therapies and therapeutic concepts for lysosomal storage diseases. Expert Opin Orphan Drugs. 2013;1(5):385-404.

87. Brown J, Brown JR, Carroll R, Glass C, Crawford BE. 22. Small molecule inhibitors of glycosaminoglycan biosynthesisas substrate optimization therapy for the mucopolysaccharidoses. Mol Genet Metab. 2010;2(99):S12.

88. Feldhammer M, Durand S, Pshezhetsky A V. Protein misfolding as an underlying molecular defect in mucopolysaccharidosis III type C. PLoS One. 2009;4(10):e7434.

89. Boado RJ. A new generation of neurobiological drugs engineered to overcome the challenges of brain drug delivery. Drug News Perspect. 2008;21(9):489-503.

90. Boado RJ, Zhou Q-H, Lu JZ, Hui EK-W, Pardridge WM. Pharmacokinetics and brain uptake of a genetically engineered bifunctional fusion antibody targeting the mouse transferrin receptor. Mol Pharm. 2009;7(1):237-244.

91. Boado RJ, Pardridge WM. Brain and Organ Uptake in the Rhesus Monkey in Vivo of Recombinant Iduronidase Compared to an Insulin Receptor Antibody-Iduronidase Fusion Protein. Mol Pharm. 2017;14(4):1271-1277.

92. Hersom M, Helms HC, Pretzer N, et al. Transferrin receptor expression and role in transendothelial transport of transferrin in cultured brain endothelial monolayers. Mol Cell Neurosci. 2016;76:59-67.

93. Böckenhoff A, Cramer S, Wölte P, et al. Comparison of five peptide vectors for improved brain delivery of the lysosomal enzyme arylsulfatase A. J Neurosci. 2014;34(9):3122-3129.

94. Wang D, El-Amouri SS, Dai M, et al. Engineering a lysosomal enzyme with a derivative of receptor-binding domain of apoE enables delivery across the bloodbrain barrier. Proc Natl Acad Sci. 2013;110(8):2999-3004.

95. El-Amouri SS, Dai M, Han J-F, Brady RO, Pan D. Normalization and improvement of CNS deficits in mice with Hurler syndrome after long-term peripheral delivery of BBB-targeted iduronidase. Mol Ther. 2014;22(12):2028-2037.

96. Farrell KB, Karpeisky A, Thamm DH, Zinnen S. Bisphosphonate conjugation for bone specific drug targeting. Bone reports. 2018;9:47-60. 
97. Schuh RS, Baldo G, Teixeira HF. Nanotechnology applied to treatment of mucopolysaccharidoses. Expert Opin Drug Deliv. 2016;13(12):1709-1718.

98. Tomatsu S, Almeciga-Diaz CJ, Montano AM, et al. Therapies for the bone in mucopolysaccharidoses. Mol Genet Metab. 2015;114(2):94-109.

99. Wang G, Mostafa NZ, Incani V, Kucharski C, Uludağ H. Bisphosphonate-decorated lipid nanoparticles designed as drug carriers for bone diseases. J Biomed Mater Res Part A. 2012;100(3):684-693.

100. Bidone J, Schuh RS, Farinon M, et al. Intra-articular nonviral gene therapy in mucopolysaccharidosis I mice. Int J Pharm. 2018;548(1):151-158.

101. Pittenger MF, Mackay AM, Beck SC, et al. Multilineage potential of adult human mesenchymal stem cells. Science. 1999;284(5411):143-147.

102. Jiang Y, Jahagirdar BN, Reinhardt RL, et al. Pluripotency of mesenchymal stem cells derived from adult marrow. Nature. 2002;418(6893):41.

103. Le Blanc K. Immunomodulatory effects of fetal and adult mesenchymal stem cells. Cytotherapy. 2003;5(6):485-489.

104. Karp JM, Leng Teo GS. Mesenchymal Stem Cell Homing: The Devil Is in the Details. Cell Stem Cell. 2009;4(3):206-216.

105. Mendt M, Rezvani K, Shpall E. Mesenchymal stem cell-derived exosomes for clinical use. Bone Marrow Transplant. 2019;54(S2):789-792. doi:10.1038/s41409019-0616-z

106. Gurunathan S, Kang M-H, Jeyaraj M, Qasim M, Kim J-H. Review of the Isolation, Characterization, Biological Function, and Multifarious Therapeutic Approaches of Exosomes. Cells. 2019;8(4):307.

107. Lema DA, Burlingham WJ. Role of exosomes in tumor and transplant immune regulation. Scand J Immunol. 2019.

108. Coutinho MF, Prata MJ, Alves S. A shortcut to the lysosome: the mannose-6phosphate-independent pathway. Mol Genet Metab. 2012;107(3):257-266.

109. Coulson-Thomas VJ, Caterson B, Kao WW-Y. Transplantation of human umbilical mesenchymal stem cells cures the corneal defects of mucopolysaccharidosis VII mice. Stem Cells. 2013;31(10):2116-2126.

110. Casciaro S, Conversano F, Ragusa A, et al. Optimal Enhancement Configuration of Silica Nanoparticles for Ultrasound Imaging and Automatic Detection at Conventional Diagnostic Frequencies. Invest Radiol. 2010;45(11):715-724.

111. Wang Z, Wang Z. Treatment of ultrasound contrast agents for gene or drug delivery. Chinese J Interv Imaging Ther. 2006;4.

112. Bors LA, Erdö F. Overcoming the Blood-Brain Barrier. Challenges and Tricks for CNS Drug Delivery. Sci Pharm. 2019;87(1):6. 
113. Burgess A, Hynynen K. Noninvasive and Targeted Drug Delivery to the Brain Using Focused Ultrasound. ACS Chem Neurosci. 2013;4(4):519-526.

114. Horodyckid C, Canney M, Vignot A, et al. Safe long-term repeated disruption of the blood-brain barrier using an implantable ultrasound device: a multiparametric study in a primate model. $J$ Neurosurg. 2017;126(4):1351-1361.

115. Hsu Y-H, Liu R-S, Lin W-L, Yuh Y-S, Lin S-P, Wong T-T. Transcranial pulsed ultrasound facilitates brain uptake of laronidase in enzyme replacement therapy for Mucopolysaccharidosis type I disease. Orphanet J Rare Dis. 2017;12(1):109.

116. Park J, Zhang Y, Vykhodtseva N, Akula JD, McDannold NJ. Targeted and reversible blood-retinal barrier disruption via focused ultrasound and microbubbles. PLoS One. 2012;7(8):e42754. 


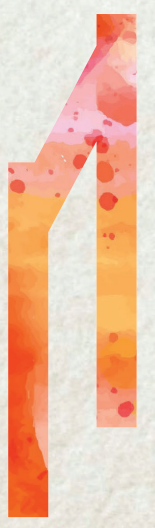




\section{PART III}

Conclusions

and Perspectives

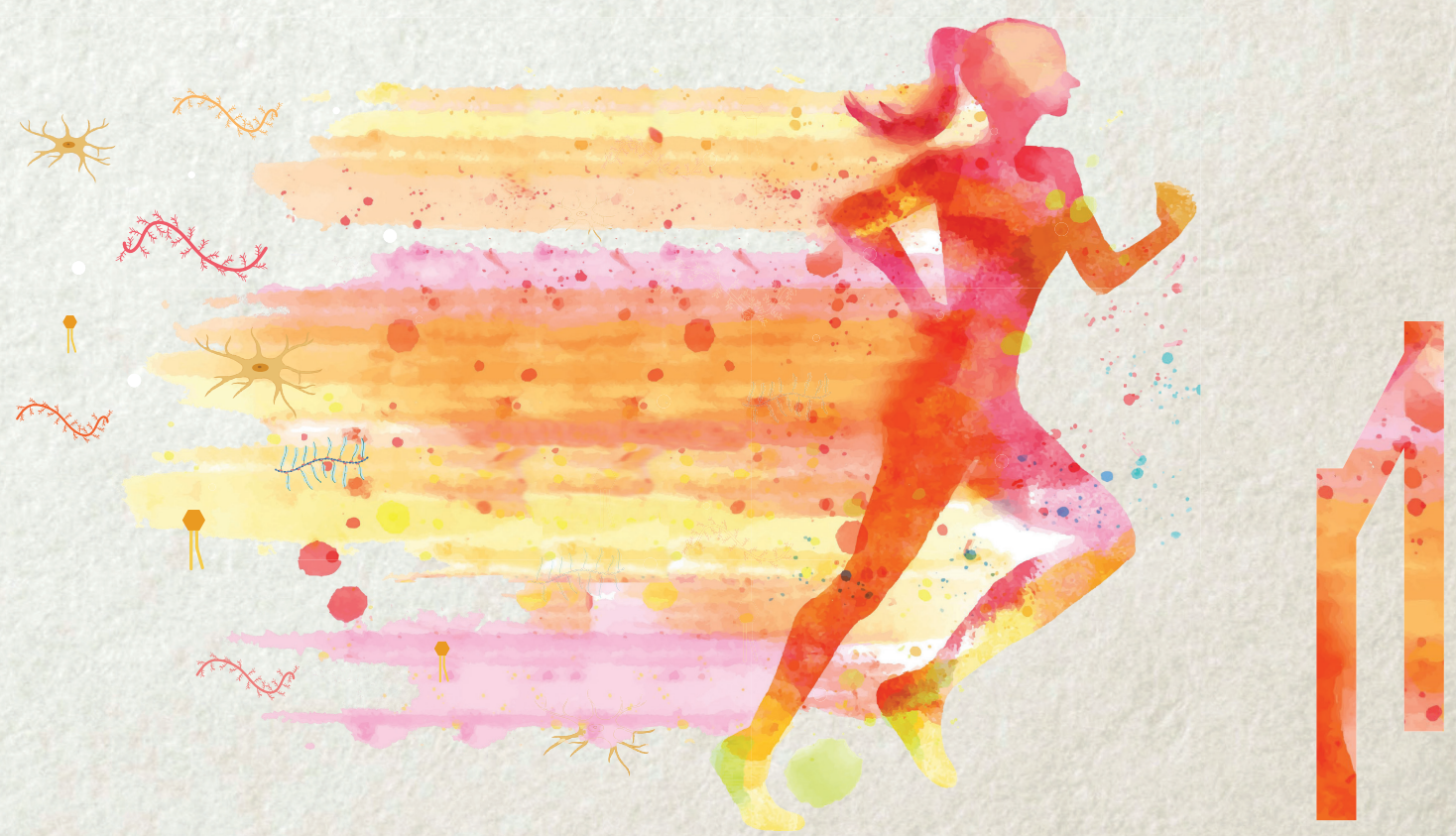




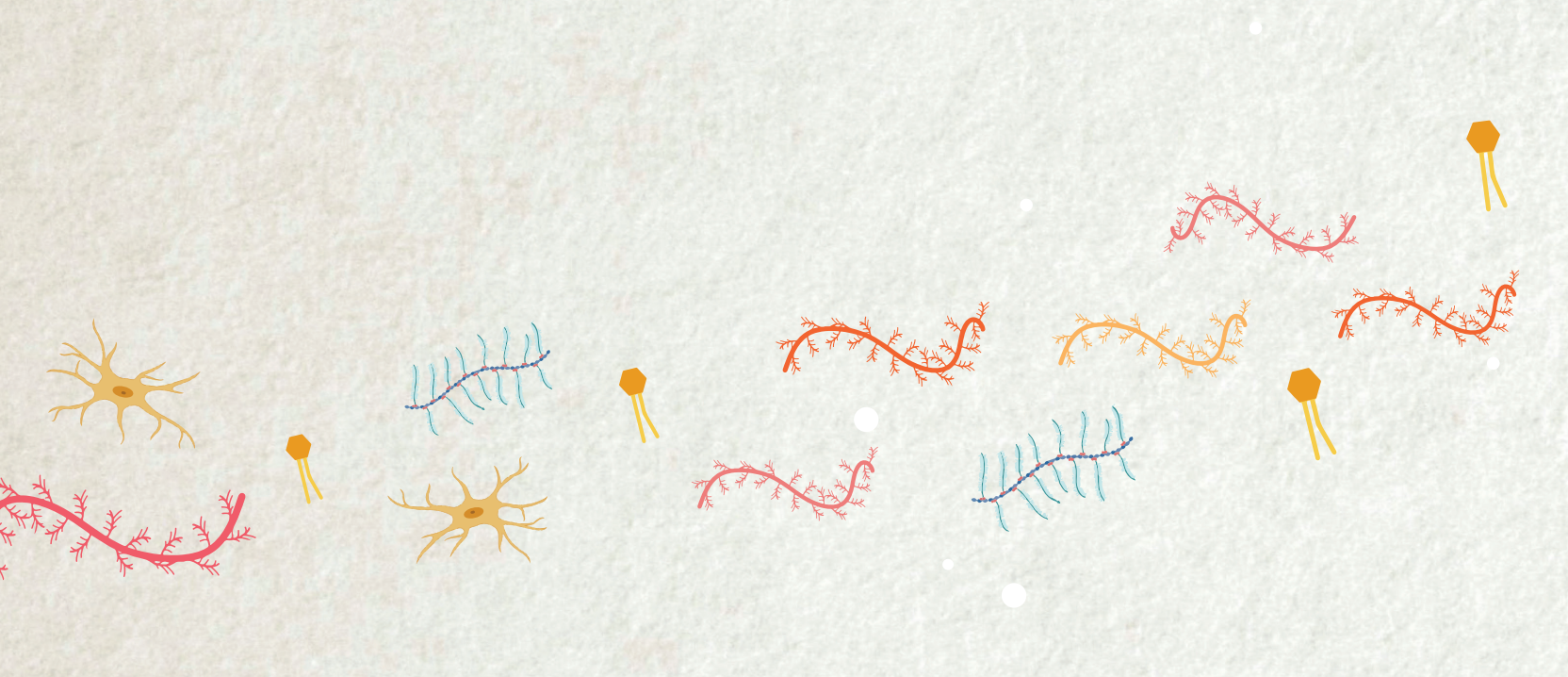



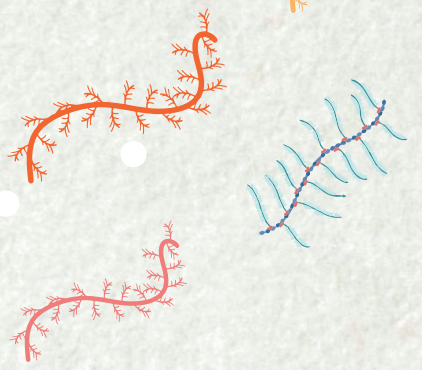

\section{CHAPTER 10}

Hematopoietic Cell Transplantation in

Inborn Errors of Metabolism: Summary,

Conclusions, and Perspectives 


\section{SUMMARY, CONCLUSIONS, AND PERSPECTIVES}

Inborn Errors of Metabolism (IEM) are a heterogeneous group of genetic diseases, including lysosomal and peroxisomal storage diseases caused by defects in genes coding for proteins involved in metabolic pathways. ${ }^{1}$ Most patients with a lysosomal storage disease appear healthy at birth. However, without treatment, all patients will inevitably follow a progressive deterioration and patients with a severe phenotype will ultimately face a premature death. Allogeneic hematopoietic cell transplantation (HCT), based on cross-correction of donorderived enzyme to the recipient, is standard care for a selected group of IEM and is considered optional for others. ${ }^{2}$ It has largely contributed to the improved survival of IEM patients suffering from disorders amenable to HCT with current long-term survival rates of over $90 \%$ upon engraftment. ${ }^{3,4}$ However, the areas of residual disease despite HCT are unfolding as patients grow older.

The objective of this thesis is to provide better insights in the residual disease of transplanted IEM patients and thereby contribute to the improvement of outcomes following HCT. The focus is mainly on Hurler syndrome patients, as this is the metabolic disorder which is transplanted most frequently. To reach this goal, long-term outcomes of HCT in patients with IEM have been analyzed in-depth. Furthermore, hypotheses regarding the pathophysiology of disease progression are explored. Additionally, this thesis may generate insights into new targets for experimental treatments and biomarkers that reflect the residual disease status of patients.

\section{PART 1: IN-DEPTH ANALYSIS OF LONG-TERM OUTCOMES AFTER HCT}

HCT has drastically improved overall survival rates of patients with certain IEM. ${ }^{2}$ Although this is a great achievement, survival alone is not enough. With over 15 years of experience in the follow-up of patients with IEM after transplantation at the Sylvia Toth Center for Multidisciplinary Follow-up after Hematopoietic Cell Transplantation, we were able to perform detailed in-depth analyses of the long-term effect of HCT in these patients and, furthermore, assess their quality of life.

\section{Hematopoietic cell transplantation in Leukodystrophies}

Chapter 2 comprehends both overall survival and long-term outcomes of 169 patients with globoid cell leukodystrophy (GLD), X-linked adrenoleukodystrophy (X-ALD) and metachromatic leukodystrophy (MLD) undergoing cord blood transplantation with a median follow-up of 6.4 years (range 0.3 - 17.6 years). Cord blood is a common donor source for 
transplantation of IEM patients because it is rapidly available and has shown to assure higher enzyme activity levels post-HCT. ${ }^{5}$ CBT in patients with Leukodystrophies (LD) showed promising early and late outcomes, especially for those who are pre-symptomatic before CBT and those who received adequately matched grafts. Early identification and treatment appeared to be important predictors of outcomes, which suggests that newborn screening (NBS) programs may further improve the outcomes. In case this is unavailable, rapid referral to a specialized center after diagnosis is of utmost importance as evidenced by the observation that symptomatic patients who were transplanted with a low performance score $(<60$ and $60-80)$, only $5 \%$ and $12 \%$, respectively, were alive with improved performance at last known follow-up. However, we also recognized that the long-term outcomes of many children are affected to various extents by their underlying disease, the transplant procedure, or a combination of both. Although nearly half of the children were able to perform at an ageappropriate level at school, many needed additional support services to achieve that level of performance, especially MLD and GLD patients. Counseling of these patients and parents is therefore of extreme importance.

The successful effect of HCT in X-ALD patients is remarkable as in these patients the genetic mutation results in a deficient peroxisomal membrane protein (the $\mathrm{ABC}$ transporter), which normally transports very long chain fatty acids (VLCFA) into the peroxisome for $\beta$-oxidation. ${ }^{6}$ Therefore, the rationale of cross-correcting is impossible in these patients and a different effect must be the reason for the therapeutic efficacy of HCT. A recent study in MLD patients might provide an attractive explanation. ${ }^{7}$ With immunofluorescence studies, Wolf et al. ${ }^{7}$, could clearly detect arylsulfatase A (ASA) in lysosomes of donor-derived monocytes/microglia in the brain of deceased transplanted MLD patients. However, ASA was not detected in oligodendrocytes nor in astrocytes, suggesting that cross-correction of enzyme from donor to recipient was not occurring. Oligodendrocyte precursors and the myelin-forming mature oligodendrocytes were however present in higher numbers in the brains of transplanted patients compared to untreated MLD patients. Transplanted patients also showed ultrastructural evidence of remyelination. This suggests HCT does support survival, proliferation, and differentiation of oligodendrocytes. The hypothesis is raised that by resetting the hematopoietic lineage in transplanted patients, the pro-inflammatory and apoptotic mode of cells surrounding the myelin is turned into an anti-inflammatory and survival mode, resulting in remyelination without cross-correction. ${ }^{7,8}$ Therefore, treating patients with anti-inflammatory agents might have additional beneficial effects and could improve the clinical outcomes of patients. ${ }^{9}$ For MPS patients, this is discussed in chapter 8 . Furthermore, if corroborated, such a 'resetting' effect of HCT might be a reason to expand the indications for HCT in neuronopathic diseases (e.g. acquired white matter disorders). 


\section{Residual disease in the Mucopolysaccharidoses}

Chapter 3 of this thesis focuses on ocular manifestations in MPS-1 patients after HCT. Corneal clouding is a hallmark feature in MPS patients. ${ }^{10}$ We observed stable corneal clouding the first years after HCT, but a rapid increase beyond three years. Visual acuity also worsened over time to a degree that could not be explained by corneal clouding alone. This also included high numbers of severe hyperopia, increased corneal central thickness, and increased intraocular pressure (IOP) as compared to healthy children. The accumulation of GAGs not only explains corneal clouding, but also helps explain other frequently observed ocular features: it increases corneal thickness, giving rise to stiffer corneas, which in turn likely diminishes the refractive capacity of the cornea and thus leads to hyperopia in these patients. It is debatable whether the measured increased IOP is truly increased IOP or merely the result of corneal stiffness, a feature which impedes the accurate measurement of IOP. The ongoing ocular pathology implies that $\alpha$-1-iduronidase (IDUA) activity levels are suboptimal despite HCT. We hypothesized that IDUA might be reaching the cornea via tear fluid, because this is the main source of nutrients for the avascular cornea. In support, although hematologic IDUA enzyme activity levels normalized after HCT, IDUA activity levels in tear fluid remained extremely low. So, concluding, ongoing ocular disease is occurring despite treatment with HCT in patients with MPS-1, resulting in impaired vision representing a burden on these patients. This finding adds to the growing body of evidence of residual disease despite current treatments. It is first of all important that clinicians inform patients undergoing HCT for MPS-1 about the evidence for ongoing disease in the eyes. Secondly, new treatments should focus on improving the ocular efficacy. Finally, retinal assessment is not routinely checked. However, our data show retinal degeneration is occurring in MPS patients despite HCT. The exact pathophysiology, however, is unknown. Future studies, with detailed evaluation of the retina in MPS patients would be helpful in acquiring more knowledge concerning this matter.

We also performed a longitudinal analysis of hearing loss in 38 MPS patients after HCT, which is described in chapter 4 . The time course of the two components of hearing, air conduction and bone conduction, moved in opposite directions. The air conducted threshold decreased significantly with a mean PTA at 1-year post-HCT of $50 \pm 0.7 \mathrm{~dB}$ to $23 \pm 11 \mathrm{~dB}$ at 13 years follow-up, indicating improved hearing. The bone conducted threshold however tended to decrease slowly over time with a mean PTA at 1-year post-HCT of $10 \pm 7 \mathrm{~dB}$ to $18 \pm 9 \mathrm{~dB}$ at 13 years follow-up. This was also reflected by the type of hearing loss, as early after HCT mostly conductive hearing loss was seen, while at later follow-up this shifted to predominantly sensorineural hearing loss and many patients already needed hearing aids at some point. It is important to realize that the mean PTA at 13 years follow-up, when most children have reached early adulthood, was at the borderline between normal hearing and 
mild hearing loss. If the conduction threshold indeed continues to worsen when patients age in addition to the natural deterioration of inner ear function by aging, this means all children will develop substantial hearing loss in adulthood, which is also important information for patients and parents. The sensorineural hearing loss was hypothesized to occur because of inner ear pathology. To provide evidence for this hypothesis, the follow-up routine has now included otoacoustic emissions measurements. Furthermore, advanced electrophysiological measurements could possibly help elucidate the pathophysiology of sensorineural in MPS. Finally, in the evaluation of new treatments, hearing loss should be included in the long-term clinical outcomes.

Previous chapters have elucidated that disease progression is occurring in both LD and MPS disorders. In addition, we were interested to what extent the residual disease impacts the health-related quality of life from the parents' perspective. Therefore, an international, multicenter cross-sectional survey, using two validated questionnaires, was conducted in 63 transplanted MPS-1 patients with at least three years of follow-up and is described in chapter 5. The functional health appeared to be significantly diminished despite HCT compared with normative data. Presumably, due to the residual disease observed in transplanted patients, especially the frequently observed skeletal disease. A higher obtained lysosomal enzyme activity level post-HCT was a predictor for superior functional health. Remarkably, psychosocial health showed much favorable outcome with similar or only slightly reduced scores compared to healthy peers. Assessment of the physical and psychosocial health can play an important role in the evaluation of the outcome of MPS patients, especially when evaluating new treatments. Finally, the parents' perspective of the processes of care were also evaluated with a third validated questionnaire. The parents were overall satisfied with the care they received. The multidisciplinary setting, with highly experienced specialists, of which the Sylvia Toth Center for Multidisciplinary Follow-up after Hematopoietic Cell Transplantation is an example, might have contributed to this high level of satisfaction.

Part 1 of this thesis demonstrates that HCT has turned MPS into a more attenuated chronic disease. However, there is still a significant disease burden which progresses over time. ${ }^{11-13} \mathrm{In}$ order to recognize the various aspects of residual disease at an early stage and allowing timely intervention, systematic follow-up of these patients is required. The MPS are rare diseases characterized by a broad range of clinical manifestations and disease progression. Therefore, guidelines on the multidisciplinary follow-up are needed, not only to assure patients get the best care, but also to enable pooling data from multiple centers to increase numbers and improve statistical power of the conclusions that are being drawn from these studies. In chapter 6 we provide an overview of the recommended evaluations in MPS patients at baseline and during follow-up after HCT as well as their proposed frequency. Ideally, all 
patients are evaluated by a multidisciplinary team experienced in MPS diseases at baseline and on a yearly basis when transplanted. As the follow-up age of the patients increases, this frequency might be reduced based on clinical judgment of the involved specialists. Detailed longitudinal data has led to increased knowledge of the long-term effects of HCT in these patients. However, as mentioned before, some aspects of the residual disease burden are still relatively unexplored (i.e. retinal disease, sensorineural hearing loss, but also the effect of surgical release on carpal tunnel syndrome and recurrence rates). Implementation of standardized follow-up can help to increase knowledge and provide reliable information to the (parents of) children, including future expectations. Furthermore, they can be a starting point for the follow-up of new therapies as autologous gene-modified hematopoietic cell transplantation.

\section{PART II: PATHOPHYSOLOGY OF DISEASE PROGRESSION AFTER HCT}

From the previous chapters, we have learned that HCT is not sufficient to fully cure MPS disorders. To design better therapies, we need to understand why and where current therapies fail. Therefore, in part 2 we focus on the pathophysiology behind the disease progression.

\section{Identifying biomarkers as proxy for long-term clinical outcomes}

Chapter 7 describes the measurement of IDUA activity in whole saliva of 20 transplanted MPS-1 patients. Saliva is a non-hematological body fluid which can be collected easily and non-invasively. We hypothesized that the extent of recovery of IDUA activity in saliva after HCT could provide more insight in what happens in non-hematological compartments. We show measurement of IDUA activity in saliva is possible and allows diagnosis of IDUA deficiency, with values a magnitude further deviating from the normal range than when assayed in corresponding dried blood spots (DBS). As IDUA activity measured in saliva is only the extracellular or excreted activity, it was an interesting observation that for controls the IDUA activity was even higher in than in DBS. The reason why extracellular IDUA activity was high remains an unanswered question but this could mean there is a potential extracellular function of IDUA (and perhaps also for other lysosomal enzymes). Furthermore, it could possibly differentiate between phenotypes, which could be helpful in the decision of treatment in newly diagnosed patients after newborn screening with mutations of which the phenotype is uncertain. More importantly, patients exhibit strikingly low values of IDUA in saliva after HCT, far below the normal range of controls, contrasting the normal IDUA activity levels in DBS. We postulate that the limited recovery of donor-derived IDUA activity in saliva after treatment reflects the situation in poorly responding non-hematological tissue compartments unveiling enzyme delivery as a weak spot of the current therapy. This 
makes salivary IDUA activity a potential biomarker for the evaluation of the effect of new therapies. Biomarkers that would allow us to evaluate the efficacy of HCT (and upcoming new therapies) in non-hematological tissues are needed. Current biomarkers, including the IDUA activity in leukocytes, are not suitable for this purpose as they are assessed in tissues of hematological origin and may not reflect enzyme availability in non-hematologic tissues.

As mentioned for LD, interest in the involvement of the immune system in the pathophysiology has also grown for MPS in the last years. Several studies in both mice and men have shown that the immune system in patients is in a pro-inflammatory state. ${ }^{14-16}$ As inflammation is a secondary manifestation of the original disease and the accumulating product in MPS, glycosaminoglycans (GAGS), also directly stimulate the immune system, ${ }^{15}$ we hypothesized that systemic inflammation markers could be interesting biomarkers for the evaluation of new therapies that aim to improve the long term clinical outcomes of MPS patients. Therefore, in chapter 8, we investigated whether systemic inflammation was present in untreated patients and to what extent systemic inflammation is ongoing after HCT up to ten years of follow-up by measuring a set of 92 biomarkers in DBS. Before transplantation, a clear increase in a large profile of mainly pro-inflammatory biomarkers was seen in MPS patients compared to controls. Other markers that were increased were related to bone homeostasis, extracellular matrix degradation, and apoptosis. Early after transplantation (up to three years), the pro-inflammatory profile was still present. However, at 10 years of follow-up, most of the inflammatory markers decreased to (near) normal levels. Five markers, however, RANKL, OPG, AXIN1, Flt3L, and SCF, which are associated with bone homeostasis, remained significantly increased and with a large difference (fold change $>2.8$ ) compared to controls up to 10 years after transplantation. RANKL, OPG, and AXIN1 had similar levels in MPS patients compared to pre-HCT. Flt3L and SCF increased over time, however an increase was also seen in controls. In this chapter, we conclude that systemic inflammation is indeed present in untreated MPS patients and is reduced upon treatment with HCT. Markers related to bone homeostasis remain elevated up to 10 years post-HCT and possibly reflect the ongoing (skeletal) disease in these patients. Therefore, they could potentially be used as biomarkers for the evaluation of residual disease in new therapies. Validation of these markers however is necessary.

Interestingly, almost 30 years ago, the minimal effect of HCT on skeletal disease and corneal clouding in MPS patients was already mentioned in most, if not all, papers describing HCT in MPS. ${ }^{17-21}$ Although transplantation protocols have improved, which has resulted in better overall survival and improved clinical outcomes, ${ }^{4,22-26}$ many of these issues are currently still disabling patients. Luckily, researchers have found their interest in the inherited metabolic disorders and new therapies are being developed and are on their way to the clinic, with 
autologous gene-transduced HCT currently being the most successful. ${ }^{27}$ To prevent another 30 years going by without real improvement in clinical long-term outcomes and quality of life for patients, the primary outcomes of clinical trials should also include the long-term outcomes of HCT and not only whether hematological enzyme activity is increased and accumulation products are decreased. Obviously, long-term outcomes are difficult to assess in a clinical trial, as they are too costly to last over 10 years and it would furthermore be very inefficient. However, in the end, these are the outcomes that will have the most impact on patients. Therefore, biomarkers that correlate with residual disease are important and can serve as proxies for long-term clinical disease. In this thesis, we have suggested some useful biomarkers for MPS, such as salival and tear fluid enzyme activity as a proxy for penetration in non-hematological tissues, and the markers RANKL, OPG, AXIN1, SCF, and F1t3L for residual (skeletal) disease. Others have also suggested the use of urinary KS as a proxy for skeletal disease, as it was correlated with growth in MPS-IV patients. ${ }^{28}$ We have not been able yet to validate these markers or correlate them to actual clinical symptoms as MPS are rare diseases and the variability within the residual disease is small. If symptoms of specific residual disease are present, almost all patients will develop them. Therefore, collaborations between centers in combination with globally standardized follow-up is of great importance to increase the numbers and to evaluate whether these markers are relatable to specific longterm clinical symptoms or disease progression in general. Furthermore, patients treated with new therapies, such as autologous gene-transduced HCT, should be assessed on these markers, preferably during care-as-usual, to evaluate whether new therapies indeed have an improved effect on these markers and thus, hopefully, long-term clinical outcomes and quality of life. Also for MLD patients identification of biomarkers as proxy for long-term clinical outcome are necessary, as the majority of patients still develop peripheral neuropathy despite HCT. ${ }^{29}$ Gene-transduced transplantation has shown to prevent the peripheral neuropathy in some, but not all, patients, however long-term follow-up studies have yet to confirm this over a longer period. ${ }^{30}$

Besides the use of biomarkers as proxy for long-term residual disease, biomarkers could also be helpful in categorizing patients within the different phenotypes often seen in patients with IEM. ${ }^{31}$ This is important as NBS programs are increasingly implemented, but genotype-phenotype correlation is not available for all mutations. ${ }^{32,33}$ Also, with newborn screening, novel mutations can be discovered of which the phenotype is yet unknown. For MLD, characterization is currently based on the age of onset or the age of onset of the index case when diagnosing a pre-symptomatic sibling. However, in NBS programs, of which pilots are running now in amongst others New York State in the United States of America, all patients are pre-symptomatic and characterization is not possible for mutations 
that do not have a clear genotype-phenotype correlation. Biomarkers that aid phenotyping are therefore needed. For MPS-1, a recent pilot study presenting the performance of a 2-tiered newborn screening method over a seven month period, showed IDUA enzyme deficiency was common. ${ }^{34}$ However, most cases were pseudo deficiency alleles, confirming the need of 2-tier testing. One MPS-1 patient with a homozygous pathogenic variant consistent with the severe phenotype was identified and referred for HCT. Although in these seven months, no patients with a novel mutation or a mutation of which the phenotype is uncertain was found, it remains an important question. With ERT also available for some of these diseases, and other experimental therapies, such as small molecule therapies, being developed, categorization of patients, especially those with and without neuronopathic disease, will become more important in the future when clinicians have to decide what the best suitable treatment is for their patients. In this thesis, we have suggested salivary IDUA activity levels as a potential biomarker for this purpose, however this must be validated in a new and larger cohort.

\section{Hurdles to 'real' cure}

In chapter 9, we summarize the knowledge we have obtained and discuss important aspects of the underlying disease and the disease progression. We note that the majority of progressive symptoms which occur in "hard-to-treat" tissues are actually "hard-to-reach" tissues. Some of the skeletal issues, which already develop pre-natal, might even be 'out-of-reach'. Two important hurdles that explain disease progression in "hard-to-reach" tissues are avascularity and tissue-specific barriers, both leading to unavailability of enzyme. The majority of disease progression in MPS occurs in connective tissue because of its avascularity. Additionally, specific barriers, like the blood-retina-barrier and the blood-brain-barrier, hamper an optimal effect of current standard therapies. Albeit the best possibility at this time, solely increasing the amount of hematological available enzyme, as is aimed for with experimental autologous gene-transduced transplantation, will, probably show better results compared to HCT but will most likely not be the holy grail in curing MPS. Therefore, the management of MPS will reasonably evolve from monotherapy to combination therapy. A combination of HCT or autologous gene-transduced transplantation and (local) therapies mentioned in this chapter could be the future for successful and complete treatment of MPS. Based on the nature and characteristics of the different MPS subtypes, each might require a different and unique treatment strategy for successful outcomes. 


\section{CONCLUDING REMARKS}

The objective of this thesis was to provide better insights in the residual disease of transplanted IEM patients. Long-term outcomes of HCT in patients with IEM have been analyzed in-depth and show disease progression is occurring in both LD and MPS patients despite HCT and, furthermore, progresses over time. Luckily, for MPS-1 patients, psychosocial quality of life is unaffected compared to healthy children. Physical quality of life however, is significantly diminished. In order to recognize the various aspects of residual disease at an early stage and allowing timely intervention, systematic follow-up of these patients is required. Therefore, we have provided an overview of the recommended evaluations in MPS patients at baseline and during follow-up after HCT as well as their proposed frequency. Ideally, all patients are evaluated by a multidisciplinary team experienced in MPS diseases, as happens at the 'Sylvia Toth Center for Multidisciplinary Follow-up After Hematopoietic Cell Transplantation'. The disease progression also emphasizes the need for better treatments. To design better therapies, we need to understand why and where current therapies fail. We note that the majority of progressive symptoms occur in "hard-to-reach" tissues. Two important hurdles that explain disease progression are avascularity and tissue-specific barriers, both leading to unavailability of enzyme. Multimodal treatment that overcome these hurdles could therefore be the future for successful and complete treatment of IEM. Finally, biomarkers that reflect long-term residual disease are necessary to evaluate these experimental therapies. This thesis has generated some potential markers for this purpose, such as salival or tear fluid enzyme activity and markers related to bone homeostasis in MPS patients. Patients treated with new therapies, such as autologous gene-transduced HCT, should be assessed on these markers to evaluate whether new therapies have an improved effect and thus, hopefully, improved longterm clinical outcomes and quality of life. 


\section{REFERENCES}

1. El-Hattab AW. Inborn errors of metabolism. Clin Perinatol. 2015 ;42(2): 413-439.

2. Tan EY, Boelens JJ, Jones SA, Wynn RF. Haematopoietic Stem Cell Transplantation in Inborn Errors of Metabolism-On Behalf of IEWP-EBMT. Front Pediatr. 2019;7:433.

3. Aldenhoven M, Jones SA, Bonney D, et al. Hematopoietic Cell Transplantation for Mucopolysaccharidosis Patients Is Safe and Effective: Results after Implementation of International Guidelines. Biol Blood Marrow Transplant. 2015;21(6):1106-1109.

4. Prasad VK, Mendizabal A, Parikh SH, et al. Unrelated donor umbilical cord blood transplantation for inherited metabolic disorders in 159 pediatric patients from a single center: influence of cellular composition of the graft on transplantation outcomes. Blood. 2008;112(7):2979-2989.

5. Boelens JJ, Aldenhoven M, Purtill D, et al. Outcomes of transplantation using various hematopoietic cell sources in children with Hurler syndrome after myeloablative conditioning. Blood. 2013;121(19).

6. Moser HW, Mahmood A, Raymond G V. X-linked adrenoleukodystrophy. Nat Rev Neurol. 2007;3(3):140.

7. Wolf NI, Breur M, Plug B, et al. Metachromatic leukodystrophy and transplantation: remyelination, no cross-correction. Ann Clin Transl Neurol. 2020;7(2):169-180.

8. Miron VE, Boyd A, Zhao J-W, et al. M2 microglia and macrophages drive oligodendrocyte differentiation during CNS remyelination. Nat Neurosci. 2013;16(9):1211.

9. Stein A, Stroobants S, Gieselmann V, D’Hooge R, Matzner U. Anti-inflammatory Therapy With Simvastatin Improves Neuroinflammation and CNS Function in a Mouse Model of Metachromatic Leukodystrophy. Mol Ther. 2015;23(7):1160-1168.

10. Ashworth JL, Biswas S, Wraith E, Lloyd IC. Mucopolysaccharidoses and the eye. Surv Ophthalmol. 2006;51(1):1-17.

11. Aldenhoven M, A van den BBT, Wynn RF, et al. Quality of life of Hurler syndrome patients after successful hematopoietic stem cell transplantation. Blood Adv. November 2017.

12. van den Broek BTA, van Egmond-Ebbeling MB, Achterberg JA, et al. Longitudinal analysis of ocular disease in children with Mucopolysaccharidosis I after hematopoietic cell transplantation. Biol Blood Marrow Transplant. November 2019.

13. B.T.A. van den Broek, Adriana L. Smit, Jaap Jan Boelens PM van H. Hearing loss in patients with Mucopolysaccharidoses after hematopoietic cell transplantation; a longitudinal analysis. Submitted. 
14. Simonaro CM, Ge Y, Eliyahu E, He X, Jepsen KJ, Schuchman EH. Involvement of the Toll-like receptor 4 pathway and use of TNF-alpha antagonists for treatment of the mucopolysaccharidoses. Proc Natl Acad Sci U S A. 2010;107(1):222-227.

15. Simonaro CM, D’Angelo M, He X, et al. Mechanism of glycosaminoglycanmediated bone and joint disease: implications for the mucopolysaccharidoses and other connective tissue diseases. Am J Pathol. 2008;172(1):112-122.

16. Opoka-Winiarska V, Jurecka A, Emeryk A, Tylki-Szymanska A. Osteoimmunology in mucopolysaccharidoses type I, II, VI and VII. Immunological regulation of the osteoarticular system in the course of metabolic inflammation. Osteoarthr Cartil. 2013;21(12):1813-1823.

17. Field RE, Buchanan JA, Copplemans MG, Aichroth PM. Bone-marrow transplantation in Hurler's syndrome. Effect on skeletal development. J Bone Joint Surg Br. 1994;76(6):975-981.

18. Huang Y, Bron AJ, Meek KM, Vellodi A, McDonald B. Ultrastructural study of the cornea in a bone marrow-transplanted Hurler syndrome patient. Exp Eye Res. 1996; 62(4): 377-387.

19. Hopwood JJ, Vellodi A, Scott HS, et al. Long-term clinical progress in bone marrow transplanted mucopolysaccharidosis type I patients with a defined genotype. $J$ Inherit Metab Dis. 1993;16(6):1024-1033.

20. Breider MA, Shull RM, Constantopoulos G. Long-term effects of bone marrow transplantation in dogs with mucopolysaccharidosis I. Am J Pathol. 1989;134(3):677.

21. Gullingsrud EO, Krivit W, Summers CG. Ocular abnormalities in the mucopolysaccharidoses after bone marrow transplantation: longer follow-up. Ophthalmology. 1998;105(6):1099-1105.

22. Boelens JJ, Rocha V, Aldenhoven M, et al. Risk factor analysis of outcomes after unrelated cord blood transplantation in patients with hurler syndrome. Biol Blood Marrow Transplant. 2009;15(5):618-625.

23. Aldenhoven M, Wynn RF, Orchard PJ, et al. Long-term outcome of Hurler syndrome patients after hematopoietic cell transplantation: an international multicenter study. Blood. 2015;125(13):2164-2172.

24. Herskhovitz E, Young E, Rainer J, et al. Bone marrow transplantation for Maroteaux-Lamy syndrome (MPS VI): long-term follow-up. J Inherit Metab Dis. 1999; 22(1): 50-62.

25. Guffon N, Bertrand Y, Forest I, Fouilhoux A, Froissart R. Bone marrow transplantation in children with Hunter syndrome: outcome after 7 to 17 years. $J$ Pediatr. 2009; 154(5): 733-737.

26. Vellodi A, Young E, Cooper A, Lidchi V, Winchester B, Wraith JE. Long-term 
follow-up following bone marrow transplantation for Hunter disease. $J$ Inherit Metab Dis. 1999; 22(5): 638-648.

27. Sawamoto K, Chen H-H, Almeciga-Diaz CJ, Mason RW, Tomatsu S. Gene therapy for Mucopolysaccharidoses. Mol Genet Metab. 2018;123(2):59-68.

28. Tomatsu S, Montano AM, Oguma T, et al. Validation of keratan sulfate level in mucopolysaccharidosis type IVA by liquid chromatography-tandem mass spectrometry. J Inherit Metab Dis. 2010;33 Suppl 3:S35-42.

29. Beerepoot S, Nierkens S, Boelens JJ, Lindemans C, Bugiani M, Wolf NI. Peripheral neuropathy in metachromatic leukodystrophy: current status and future perspective. Orphanet J Rare Dis. 2019;14(1):240.

30. Sessa M, Lorioli L, Fumagalli F, et al. Lentiviral haemopoietic stem-cell gene therapy in early-onset metachromatic leukodystrophy: an ad-hoc analysis of a nonrandomised, open-label, phase 1/2 trial. Lancet. 2016;388(10043):476-487.

31. Saudubray J-M, Garcia-Cazorla A. Inborn Errors of Metabolism Overview: Pathophysiology, Manifestations, Evaluation, and Management. Pediatr Clin North Am. 2018; 65(2): 179-208.

32. Clarke LA, Giugliani R, Guffon N, et al. Genotype-phenotype relationships in mucopolysaccharidosis type I (MPS I): Insights from the International MPS I Registry. Clin Genet. 2019;96(4):281-289.

33. Elgun S, Waibel J, Kehrer C, et al. Phenotypic variation between siblings with Metachromatic Leukodystrophy. Orphanet J Rare Dis. 2019;14(1):136.

34. Hall PL, Sanchez R, Hagar AF, Jerris SC, Wittenauer A, Wilcox WR. TwoTiered Newborn Screening with Post-Analytical Tools for Pompe Disease and Mucopolysaccharidosis Type I Results in Performance Improvement and Future Direction. Int J neonatal Screen. 2020;6(1). 


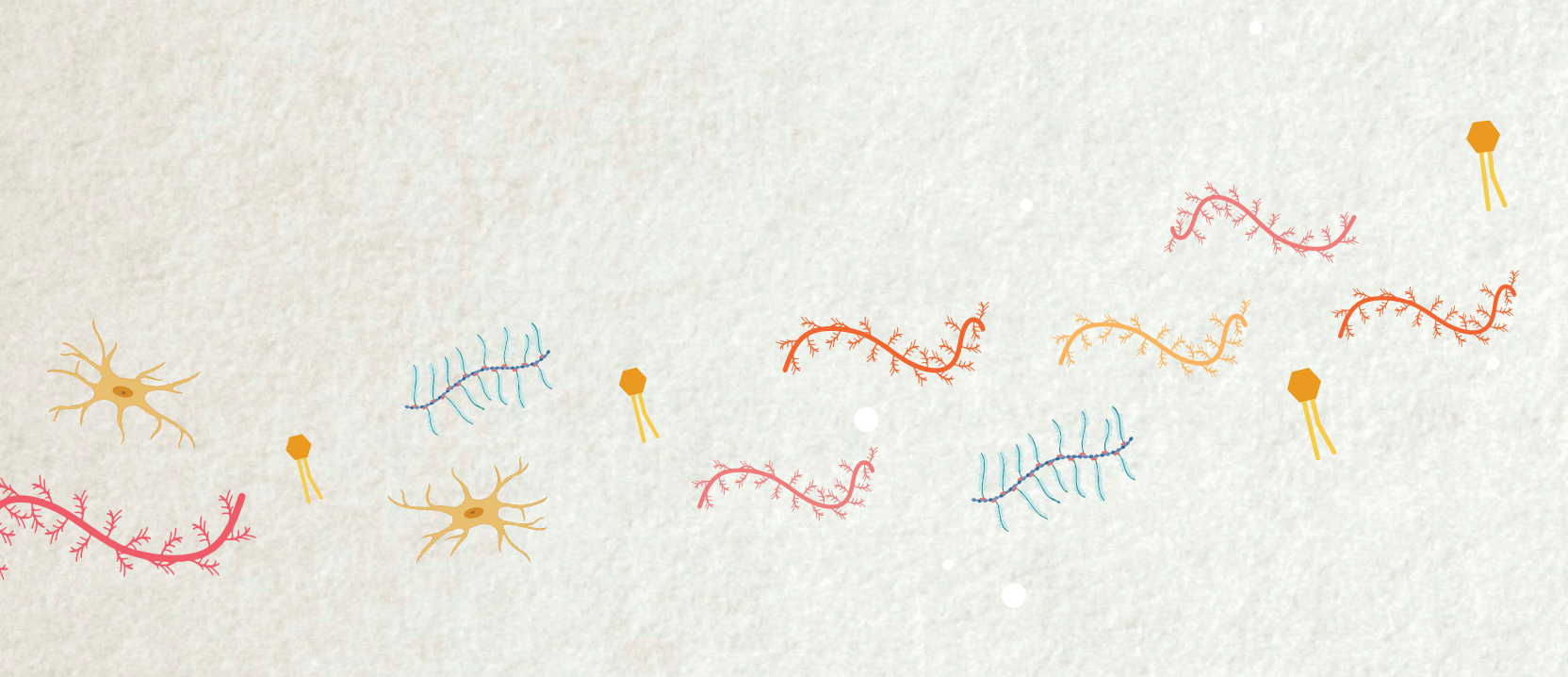



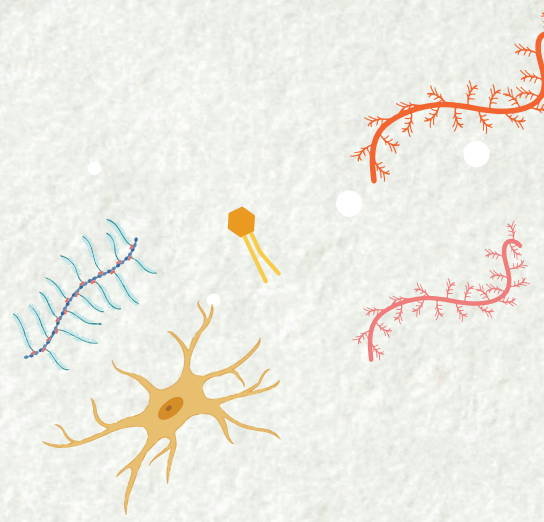

\section{CHAPTER 11}

Nederlandse Samenvatting 
Aangeboren erfelijke stofwisselingsziekten bestaan uit verschillende genetische aandoeningen, waaronder de lysosomale en peroxisomale stapelingsziekten. Bij patiënten met een stapelingsziekte werkt door een genetisch defect een specifiek enzym wat de afvalstoffen van een cel afbreekt niet meer, met stapeling van de afvalstoffen in de cel als gevolg. Door deze stapeling kunnen cellen niet goed meer functioneren en wordt een reeks van pathologische processen in gang gezet. De meeste patiënten met een stapelingsziekte hebben weinig tot geen symptomen bij de geboorte. De ontwikkeling van het kind zal echter een stilstand laten zien, gevolgd door een regressie. Kinderen met een stapelingsziekte kunnen in bijna alle organen progressieve klachten hebben, zoals malformaties van het skelet, blindheid, doofheid, mentale retardatie, psychomotore retardatie, en levensbedreigende cardiale en respiratoire complicaties. Zonder behandeling sterven kinderen met de ernstigere fenotypes al op jonge leeftijd ( $<10$ jaar).

In 1980 werd voor het eerst een patiënt met een lysosomale stapelingsziekte (Mucopolysaccharidose type 1; MPS-1) behandeld met een donorstamceltransplantatie. Hiermee werd de ziekte afgeremd en in een aantal organen kon zelfs verdere achteruitgang voorkomen worden. De werking van een donorstamceltransplantatie is berust op het feit dat de stamcellen van de donor het genetisch defect niet hebben en daardoor, na toediening in patiënten, het missende enzym kunnen produceren en zo een continue bron van enzym in de patiënt vormen. Het enzym wordt door de donorcellen afgegeven aan het bloed en kan vervolgens door de cellen van de patiënt opgenomen en gebruikt worden om de afvalstoffen alsnog te verwerken. Door behandeling met een donorstamceltransplantatie is de levensverwachting van deze kinderen enorm toegenomen. Echter, nu patiënten voor langere tijd gevolgd zijn, wordt steeds duidelijker dat patiënten nog 'restziekte' hebben in bepaalde organen (bijvoorbeeld het skelet of het perifere zenuwstelsel).

Het doel van dit proefschrift is om betere inzichten te geven in de restziekte bij kinderen met een aangeboren erfelijke stofwisselingsziekte die behandeld zijn met een donorstamceltransplantatie. De focus ligt met name op patiënten met MPS-1, aangezien dit de lysosomale stapelingsziekte is die het vaakst behandeld wordt met een donorstamceltransplantatie en waar we dus de meeste informatie over hebben. Om dit doel te bereiken hebben we ten eerste een aantal van de late uitkomsten geanalyseerd (zoals onder andere hoornvliestroebeling, visus, gehoor, en kwaliteit van leven). Daarnaast hebben we een aantal hypotheses die de restziekte zouden kunnen verklaren onderzocht. Ook hebben we de huidige inzichten gebundeld en samengevat wat op dit moment nog de obstakels zijn waardoor de huidige therapie niet toereikend is. Tevens hebben we de op dit moment experimentele therapieën kritisch beoordeeld om te bepalen in hoeverre ze deze obstakels kunnen overkomen. Ten slotte hebben we onderzocht of er relatief makkelijk te verkrijgen 
biomarkers bestaan die de status van de restziekte reflecteren zodat nieuwe therapieën sneller geëvalueerd kunnen worden op hun effect op de restziekte.

\section{Deel I: Analyse van late uitkomsten in patiënten met een aangeboren erfelijke stofwisselingsziekte die behandeld zijn met een donorstamceltransplantatie}

In deel 1 van dit proefschrift worden de (longitudinale) analyses van een aantal late uitkomsten beschreven. Hoofstuk 2 gaat specifiek over de overleving en late uitkomsten na behandeling met een (navelstrengbloed) donorstamceltransplantatie bij patiënten met leukodystrofieën, een specifieke groep stapelingsziekten. Navelstrengbloed is een veel gebruikte bron voor donorstamceltransplantaties aangezien het vaak snel beschikbaar is en daarnaast bij de meerderheid van de patiënten hogere enzymactiviteitniveaus na transplantatie laat zien, waarvan bewezen is dat het voor betere uitkomsten zorgt. Bij patiënten met een leukodystrofie liet een donorstamceltransplantatie veelbelovende overlevingskansen en late uitkomsten zien, met name bij patiënten die nog geen symptomen hadden voor de behandeling en patiënten die goed waren gematcht met de donor. Vroege identificatie en behandeling is dus belangrijk om betere uitkomsten te bereiken. Hielprikscreening zou hier enorm aan bijdragen. Wanneer dit niet mogelijk is, is een snelle doorverwijzing naar een gespecialiseerd centrum heel belangrijk. Toch zagen we ook minder goede effecten op een aantal late uitkomsten. Veel kinderen hadden bijvoorbeeld extra ondersteuning op school nodig. Het is belangrijk om deze uitkomsten goed te communiceren met patiënten en hun ouders wanneer het effect van de behandeling met hen besproken wordt.

Hoofdstuk 3 beschrijft oogheelkundige manifestaties in MPS-1 patiënten na donorstamceltransplantatie. Hoornvliestroebeling is een veel gezien symptoom in MPS patiënten. Na behandeling met een donorstamceltransplantatie, zagen we een relatief stabiele troebeling in de eerste 3 jaar na transplantatie. Daarna nam deze troebeling echter snel toe. De visus ging ook met de jaren achteruit, welke niet geheel verklaard kon worden door de hoornvliestroebeling. We zagen een hoog percentage met ernstige bijziendheid, toegenomen dikte van het hoornvlies en toegenomen intra-oculaire oogdruk. Het feit dat we deze symptomen nog steeds zien na transplantatie vermoed dat de enzymactiviteit levels in het oog niet optimaal zijn. We onderzochten daarom wat de enzymactiviteit in het traanvocht van patiënten was en vergeleken deze met gezonde controles. Aangezien het hoornvlies geen bloedvaten bevat en wordt gevoed door het traanvocht, vermoedden we dat dit de bron van enzym voor de cornea moest zijn. Interessant genoeg zagen we dat de enzymactiviteit niveaus in patiënten extreem laag bleven na transplantatie, terwijl de enzymactiviteitniveaus in hun bloed wel genormaliseerd waren. Gezonde controles lieten normale enzymactiviteitniveaus 
in traanvocht zien wanneer deze vergeleken werden met de enzymactiviteitniveaus in het bloed, dit bevestigde dat enzym vanuit het bloed niet in het traanvocht terecht kwam. De klinische bevindingen van deze studie zijn belangrijk om te bespreken met patiënten en ouders wanneer de effecten van een donorstamceltransplantatie worden besproken. Daarnaast is het belangrijk dat nieuwe therapieën zich richten op het verbeteren van de beschikbaarheid van het enzym in het oog.

Naast de oogheelkundige manifestaties hebben we ook het gehoor over tijd geanalyseerd in MPS patiënten na een donorstamceltransplantatie. Dit staat beschreven in hoofdstuk 4. Het gehoor bestaat uit twee componenten, namelijk luchtgeleiding en botgeleiding. Deze twee componenten lieten een tegenovergesteld beloop zien over tijd na behandeling met een donorstamceltransplantatie. Waar luchtgeleiding significant verbeterde, liet botgeleiding een langzame achteruitgang zien. Dit ging samen met het type gehoorverlies dat gezien werd. Vroeg na transplantatie was dit voornamelijk conductief gehoorverlies (wat correleert met slechte luchtgeleiding), later na transplantatie was dit voornamelijk sensorineuronaal gehoorverlies. Veel patiënten kregen een gehoorapparaat. Belangrijk is dat ondanks dat luchtgeleiding significant verbeterde, het gemiddelde gehoorverlies op 13 jaar na transplantatie op de grens van normaal gehoor en matig gehoorverlies lag. Dit betekent dat deze kinderen in hun volwassen leven waarschijnlijk allemaal substantieel gehoorverlies zullen ontwikkelen, aangezien het gehoor ook in gezonde personen verslechterd met de leeftijd. Ook hier geldt dat deze informatie belangrijk is om te delen met patiënten en hun ouders bij het bespreken van de verwachte effecten van de transplantatie. Daarnaast ondersteunt deze bevinding het gegeven dat huidige therapie ontoereikend is en ziekteprogressie gezien wordt in deze patiënten ondanks behandeling met een donorstamceltransplantatie. Nieuwe therapieën moeten zich richten op het verbeteren van deze late uitkomsten, waaronder het gehoor.

De voorgaande hoofdstukken hebben laten zien dat restziekte en ziekteprogressie voorkomen in zowel leukodystrofie als MPS patiënten. Om te weten wat voor impact deze restziekte heeft op de kwaliteit van leven van MPS patiënten worden in hoofdstuk 5 de resultaten van een internationale studie over de aan de gezondheid gerelateerde kwaliteit van leven van getransplanteerde MPS-1 patiënten beschreven, vanuit het perspectief van de ouders. Op basis van gevalideerde vragenlijsten blijkt dat ondanks stamceltransplantatie de fysieke gezondheid van patiënten met MPS-1 aanzienlijk verminderd is ten opzichte van een normatieve populatie. Dit heeft waarschijnlijk te maken met de aanzienlijke restziekte die wordt gezien bij de getransplanteerde patiënten. Een hoger enzymactiviteitsniveau verkregen na transplantatie is voorspellend voor een betere fysieke gezondheid. Interessant genoeg liet de psychosociale gezondheid een veel gunstiger uitkomst zien, met normale of slechts minimale verminderde scores vergeleken met gezonde kinderen. Op basis van een derde 
gevalideerde vragenlijst blijken ouders over het algemeen zeer tevreden met de zorg die zij ontvingen van hun transplantatiecentrum. Het feit dat alle patiënten werden gemonitord in een multidisciplinaire setting met zeer ervaren specialisten, waar het 'Sylvia Toth Centrum voor Multidisciplinaire Follow-up na Stamceltransplantatie' een voorbeeld van is, kan hebben bijgedragen aan dit hoge niveau van tevredenheid.

Deel 1 van dit proefschrift laat zien dat een donorstamceltransplantatie MPS in een chronische ziekte doet veranderen. De last van de restziekte is echter nog steeds substantieel.

Om de verschillende aspecten van de restziekte die kunnen optreden bij getransplanteerde MPS-1 patiënten tijdig te herkennen, zodat vroege interventie mogelijk is, is een systematische follow-up vereist. Omdat de MPS zeldzame ziektes zijn met een grote range aan klinische symptomen, zijn richtlijnen nodig. Dit niet alleen om de beste zorg voor patiënten te garanderen, maar ook om de data van verschillende (internationale) ziekenhuizen te kunnen bundelen om zo betere conclusies te kunnen trekken met sterkere statistische zekerheid. Hoofdstuk 6 biedt een overzicht van de geadviseerde evaluaties bij MPS patiënten zowel voorafgaand aan als gedurende follow-up na stamceltransplantatie. Idealiter worden alle patiënten voorafgaand aan de transplantatie en jaarlijks erna geëvalueerd door een multidisciplinair team dat gespecialiseerd is in MPS aandoeningen. Als de follow-up leeftijd van de patiënt toeneemt, kan deze frequentie zo mogelijk verminderd worden op basis van de klinische beoordeling van de betrokken specialisten.

\section{Deel II: Pathofysiologie van ziekteprogressie na donorstamceltransplantatie}

Voorgaande hoofdstukken hebben laten zien dat een donorstamceltransplantatie MPS niet volledig kan genezen. Om betere therapieën te ontwikkelen moeten we beter begrijpen waarom de huidige therapie niet toereikend is. Deel 2 van dit proefschrift is hier dan ook op gericht.

Hoofdstuk 7 beschrijft het meten van IDUA activiteit in speeksel van MPS-1 patiënten. IDUA is het deficiënte enzym in deze patiënten. Speeksel is net zoals bloed een lichaamsvloeistof, maar is makkelijker te verkrijgen. Onze hypothese was dat de hoogte van enzymactiviteit in speeksel na transplantatie inzichten kon geven die mogelijk overeen komen met andere lichaamsvloeistoffen/weefsels niet zijnde bloed. Eerst hebben we gekeken naar de enzymactiviteit in speeksel van gezonde personen. Dit bleek hoger te zijn dan enzymactiviteit in bloed van gezonde controles. IDUA activiteit in speeksel van patiënten die nog niet behandeld waren was inderdaad erg laag. Het verschil in IDUA activiteit tussen controles en patiënten was groter in speeksel dan in bloed. Interessant genoeg, zagen we in met donorstamceltransplantatie behandelde patiënten dat de IDUA activiteit in speeksel 
iets verbeterde, maar nog steeds heel laag bleef, terwijl de IDUA activiteit in het bloed van deze patiënten normaliseerde. Hiermee laten we zien dat het transport van enzym vanuit het bloed niet overal optimaal verloopt. Enzymactiviteit in speeksel zou hiermee een potentiële biomarker kunnen zijn, om het effect van nieuwe therapieën te meten en te evalueren of deze therapieën een betere penetrantie hebben in speeksel en daarmee mogelijk ook in andere weefsels. Met het meten van de IDUA activiteit in speeksel konden we ook patiënten met een milder fenotype, die mogelijk met minder ingrijpende therapie zoals enzymvervangingstherapie kunnen worden behandeld, onderscheiden. Dit resultaat moet nog wel in een groter cohort gevalideerd worden.

Naast de hypothese dat enzym niet alle weefsels even goed lijkt te bereiken, was er op basis van literatuur de hypothese dat chronische inflammatie onderdeel van de ziekte is en ook voor ziekteprogressie kan zorgen. Mogelijk zouden inflammatiemarkers in het bloed goede biomarkers zijn om nieuwe therapieën te evalueren op hun effect op restziekte. Om dit te onder zoeken hebben we in hoofdstuk 8 een set van 92 biomarkers gemeten in het bloed van MPS patiënten, zowel vóór donorstamceltransplantatie als op 1, 3, en 10 jaar na transplantatie. Vóór donorstamceltransplantatie was er duidelijk een verschil tussen controles en patiënten. Met name pro-inflammatoire markers waren verhoogd in patiënten. Andere markers die verhoogd waren, waren markers die te maken hebben met bothomeostase, degradatie van extracellulaire matrix, en apoptose. Vroeg na transplantatie (op 1 en 3 jaar post-transplantatie) was dit pro-inflammatoire profiel nog steeds aanwezig in patiënten. Op 10 jaar na transplantatie echter, waren de meeste inflammatoire markers gedaald naar (bijna) normaal. Echter, vijf markers (RANKL, OPG, AXIN1, Flt3L, en SCF), welke allen gerelateerd kunnen worden aan bothomeostase, bleven zowel significant als met een groot verschil verhoogd in patiënten, vergeleken met controles na 10 jaar follow-up. Aangezien deze markers allen aan bothomeostase gerelateerd zijn, zouden ze potentieel als biomarker kunnen fungeren voor (skeletale) restziekte. Dit moet echter nog gevalideerd worden in een ander cohort MPS patiënten.

In hoofdstuk 9 hebben we onze opgedane kennis gebundeld en bediscussiëren we belangrijke aspecten van de onderliggende ziekte en restziekte na behandeling in MPS patiënten. We merken op dat het merendeel van de restziekte en ziekteprogressie aanwezig in 'moeilijkte-behandelen' weefsels eigenlijk 'moeilijk-te-bereiken' weefsels zijn. Twee belangrijke obstakels die we steeds tegenkomen zijn avasculariteit en weefselspecifieke barrières, welke beiden leiden tot weinig of geen beschikbaarheid van het enzym in die weefsels. Het grootste gedeelte van de klachten in MPS wordt gezien in bindweefsel vanwege de avasculariteit. Daarnaast belemmeren een aantal weefselspecifieke barrières zoals de bloed-brein-barrière en de bloed-retina-barrière optimale penetrantie van enzym naar het 
brein en de retina. Op dit moment is de meest gevorderde nieuwe therapie de genetisch gemodificeerde autologe stamceltransplantatie, waarbij het genetisch defect in de stamcellen van de patiënt zelf gecorrigeerd wordt en tevens het gen wat het enzym produceert een boost krijgt, waardoor er bij patiënten een 10x hogere enzymactiviteit in het bloed aanwezig is. Hoewel dit waarschijnlijk de restziekte in een zekere mate zal verminderen, zal ook deze therapie, gezien de huidige inzichten, de restziekte niet in zijn geheel kunnen behandelen. De behandeling van MPS zal potentieel evolueren van een monotherapie op dit moment, naar een combitherapie waarbij systemische therapie mogelijk moet worden aangevuld met lokale therapieën om de obstakels waar men op dit moment met systemische therapieën tegenaan loopt te overkomen. Het kan zijn dat ieder type MPS zijn eigen unieke behandelingsstrategie krijgt gezien de aard en karakteristieken van de verschillende types, om zo de beste uitkomsten voor patiënten te garanderen.

Ten slotte worden in hoofdstuk 10 de belangrijkste conclusies die uit dit proefschrift getrokken kunnen worden besproken. Het doel van het proefschrift was betere inzichten te krijgen in de restziekte van getransplanteerde patiënten met een aangeboren erfelijke stofwisselingsziekte. We hebben late uitkomsten geanalyseerd en op basis daarvan kunnen we de conclusie trekken dat zowel in leukodystrofie als MPS patiënten ziekteprogressie optreedt ondanks behandeling met donorstamceltransplantatie. Gelukkig konden we concluderen dat de psychosociale gezondheid van MPS patiënten niet verminderd was. De fysieke gezondheid was echter wel significant verminderd ten opzichte van gezonde kinderen. Om de verschillende aspecten van de restziekte tijdig te herkennen en te kunnen behandelen is een systematische followup nodig, het liefst door een multidisciplinair team met expertise in MPS zoals bijvoorbeeld in het 'Sylvia Toth Centrum voor Multidisciplinaire Follow-Up na Stamceltransplantatie'. Een overzicht voor deze follow-up werd opgesteld. De ziekteprogressie benadrukt ook de noodzaak voor betere therapieën. Om deze te kunnen ontwikkelen, moeten we begrijpen waarom de huidige therapieën niet toereikend zijn. We hebben geobserveerd dat de meeste ziekteprogressie plaatsvindt in 'moeilijk-te-bereiken' weefsels. Twee belangrijke obstakels zijn avasculariteit en weefselspecifieke barrières, die beiden leiden tot onbeschikbaarheid van enzym in bepaalde weefsels. Combinatietherapie die deze obstakels overkomen zou de toekomst voor een complete behandeling van MPS kunnen betekenen. Ten slotte, biomarkers die restziekte reflecteren zijn nodig om de nieuwe therapieën efficiënt te evalueren. Dit proefschrift heeft hiervoor een aantal potentiële kandidaten geleverd voor MPS patiënten, zoals enzymactiviteit in speeksel en traanvocht, en markers uit het bloed die gerelateerd zijn aan bothomeostase. Bij patiënten die behandeld worden met experimentele therapieën, zoals genetisch gemodificeerde autologe stamceltransplantatie, zouden deze markers bekeken moeten worden om het effect van de behandeling op restziekte efficiënter te evalueren. 


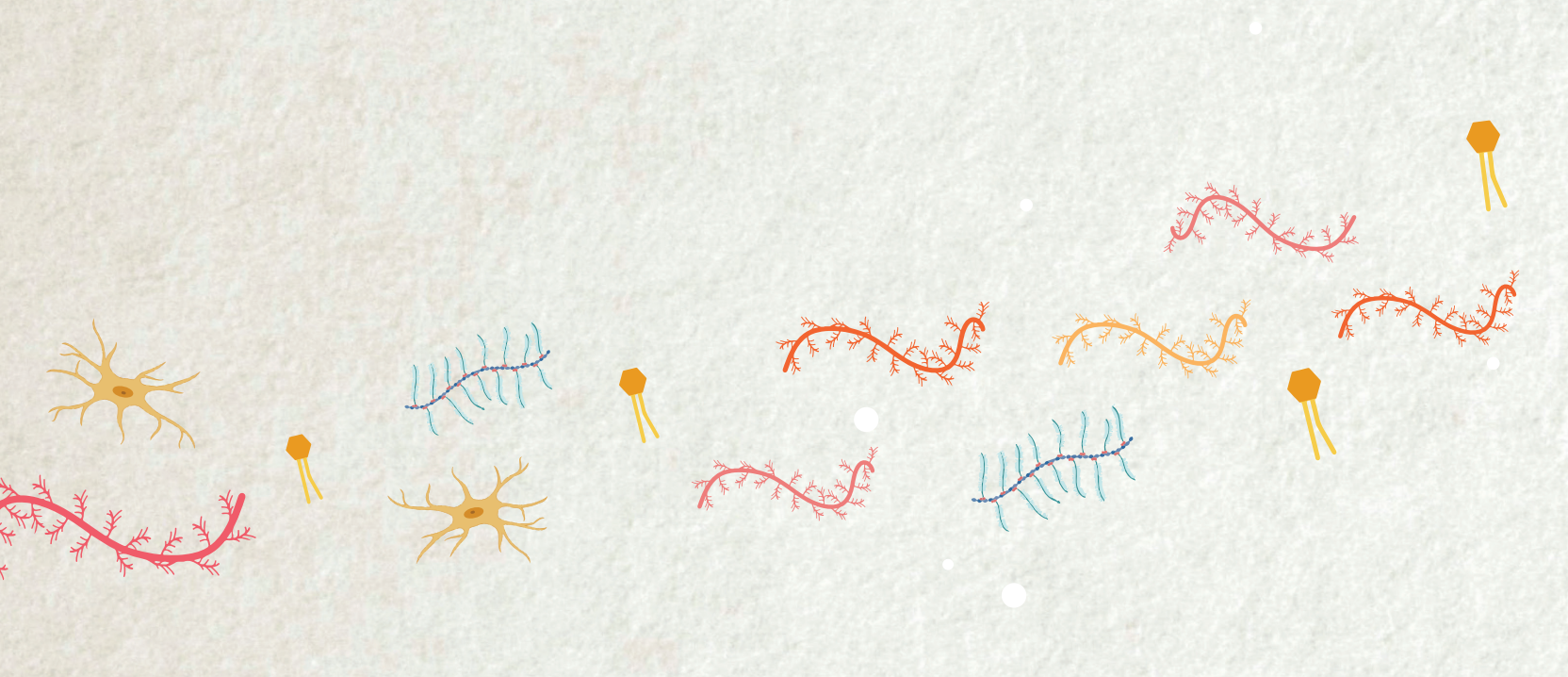



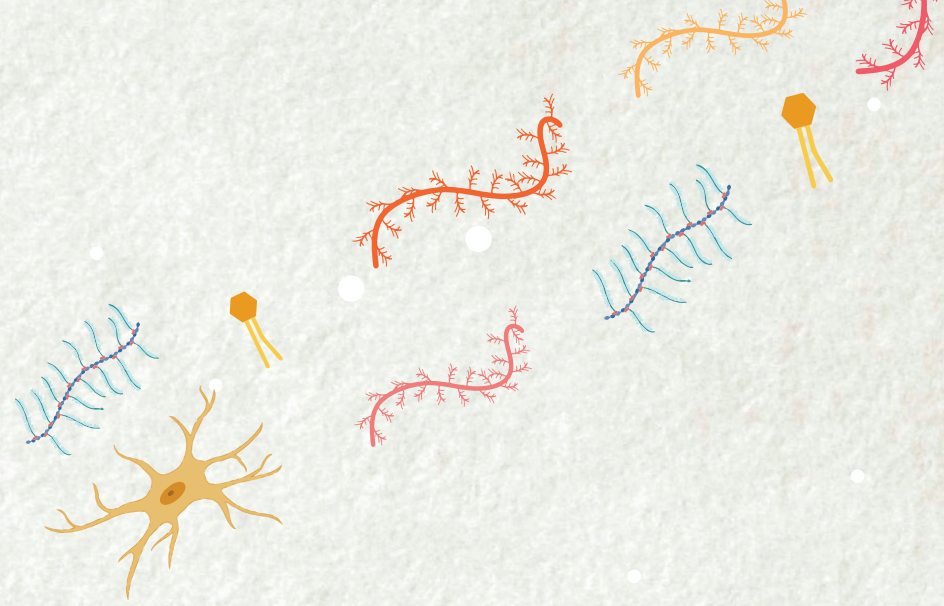

\title{
CHAPTER 12
}

Appendices

\author{
Curriculum Vitae \\ List of Publications
}

Dankwoord 
212 Chapter 12 


\section{CURRICULUM VITAE}

Brigitte van den Broek was born on September $14^{\text {th }}, 1990$ in Roosendaal, the Netherlands. She attended secondary school at the Norbertuscollege in Roosendaal where she graduated in 2008. That same year, she started her medical studies at the Utrecht University, in Utrecht, the Netherlands. Inspired by her well-traveled aunt, she took a gap year of 18 months between her bachelors' and masters' degree. She first worked as a camp counselor at a Jewish summer camp in Great Barrington, Massachusetts, USA and went traveling for 8 months thereafter through Russia,

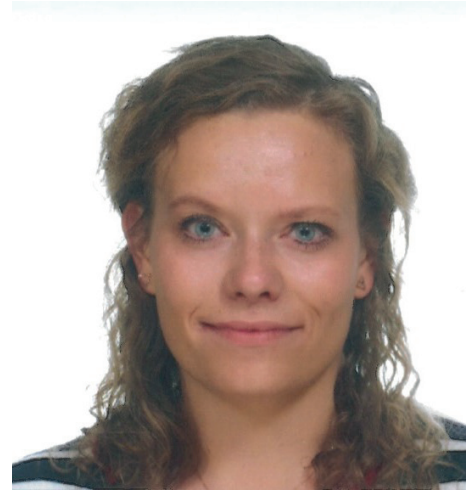
Asia, Australasia, and Western USA. She learned Spanish in Merida, Mexico and continued her traveling for another 7 months through Northern, Central, and South America. Her broad interest in global health resulted in several internships abroad, including Ophthalmology in Manipal, India, Tropical Medicine in Katete, Zambia, and Social Medicine in San Diego, California, USA. In her final year of medicine, she participated in the multidisciplinary dedicated transition year focusing on the care for acutely compromised patients. Her interest in research in the immunology field grew in her final year of medicine when she studied tuberculosis under the supervision of dr. S. Nierkens and dr. R.W. Hofland. After obtaining her medical degree in 2016, she was appointed PhD-student at the University Medical Center Utrecht and the Utrecht University under the supervision of prof. dr. J.J. Boelens, dr. P.M. van Hasselt, prof. d.r. N.M. Verhoeven-Duif, and dr. S. Nierkens which resulted in this thesis. Her researched focused on the late effects of hematopoietic cell transplantation in inborn errors of metabolism. During her PhD-project, she was elected as a board member for the Research and Education Committee of the University Medical Center Utrecht. Furthermore, she combined her $\mathrm{PhD}$ thesis with a post-graduate Masters degree in Clinical Epidemiology and Medical Statistics. Brigitte currently works as a resident in Pulmonology at the Curaçao Medical Center in Willemstad, Curaçao. As of January 2021, she will start her training to become a military doctor for the Royal Netherlands Air Force. 


\section{LIST OF PUBLICATIONS}

\section{This thesis:}

van den Broek BTA, Smit AL, Boelens JJ, van Hassel PM. Hearing Loss in Patients with Mucopolysaccharidoses after Hematopoietic Cell Transplantation; a Longitudinal Analysis. Journal of Inherited Metabolic Disease. 2020 Under review.

van den Broek BTA, van Doorn J, Hegeman CV, et al. Hurdles in Treating Hurler's Disease: Potential Routes to Achieve a 'Real' Cure. Blood Advances. 2020 Under review.

van den Broek BTA, van Egmond-Ebbeling MB, Achterberg JA, et al. Longitudinal Analysis of Ocular Disease in Children with Mucopolysaccharidosis I after Hematopoietic Cell Transplantation. Biology of Blood and Marrow Transplantation. 2019 Epub ahead of print.

van den Broek BTA, van Doorn J, Geboers AJ, et al. Salivary $\alpha$-iduronidase activity as a potential new biomarker for the diagnosis and monitoring the effect of therapy in mucopolysaccharidosis type I. Biology of Blood and Marrow Transplantation. 2018; 24(9), 1808-1813.

van den Broek BTA, Page K, Paviglianiti A, et al. Early and late outcomes after cord blood transplantation for pediatric patients with inherited leukodystrophies. Blood advances. 2018; 2(1), 49-60.

van den Broek BTA, Aldenhoven M, Wynn RF, et al. Quality of life of Hurler syndrome patients after successful hematopoietic stem cell transplantation. Blood advances. 2017; 1(24), 2236-2242. 


\section{Other:}

Hofland RW, Bossink AW, Nierkens S, et al. QuantiFERON-plus does not discriminate between active and latent tuberculosis. Infectious Diseases. 2018; 50(6), 479-482.

Kuper WF, Van Alfen C, Van Eck L, Van Den Broek BTA, et al. A case of unexpected adultonset neurologic decline in CLN3-associated retinal degeneration. JAMA ophthalmology, 2017; 135(12), 1451-1453.

Nouwen MJ, Klijn FA, van den Broek BTA, Slooter AJ. Emotional consequences of intensive care unit delirium and delusional memories after intensive care unit admission: a systematic review. Journal of critical care. 2012; 27(2), 199-211. 


\section{DANKWOORD}

\section{Every individual matters. Every individual has a role to play.}

Every individual makes a difference. - Dr. Jane Goodall

Het moment is eindelijk daar, het proefschrift is echt officieel af! Ik kijk met genoegen terug op de afgelopen drieënhalf jaar waarin ik ontelbaar veel heb geleerd, zowel op wetenschappelijk als persoonlijk vlak en ben daar een heleboel mensen erg dankbaar voor want promoveren doe je zéker niet alleen. Zonder al deze mensen zou dit proefschrift er niet zijn geweest. Op alle manuscripten kreeg ik standaard het comment: 'Mag korter!', maar om jullie te bedanken neem ik graag alle woorden die ik denk nodig te hebben. Derhalve, bij voorbaat vast excuses als het dankwoord wat lang van stof is en tevens excuses aan hen die ik nu alsnog niet persoonlijk heb kunnen benoemen, weet dat ik ook jullie dankbaar ben!

Om te beginnen wil ik graag alle patiënten en families bedanken die hebben meegewerkt aan de onderzoeken. Zonder jullie was het nooit mogelijk geweest dit proefschrift te schrijven. Het is dan ook zowel voor als door jullie! Ik heb ontzettend veel van jullie geleerd en heb erg veel bewondering voor de manier waarop jullie in het leven staan.

Beste Peter, ontzettend bedankt voor jouw steun en begeleiding de afgelopen 3,5 jaar. Van jou heb ik echt geleerd hoe een goed verhaal op te schrijven. Hoewel ik het soms al lang goed vond, wist je me altijd te motiveren, meestal met een of andere metafoor, het verhaal nog nét ietsje te verduidelijken. Ik ken weinig mensen die zoals jij out-of-the-box durven te denken. Onze beider ietwat chaotische breinen zorgden soms voor niet kloppende of vergeten afspraken / taken waarbij ik dan moest benadrukken dat ik niet je secretaresse was en zéker ook niet die ambitie had. Daarentegen probeerde je altijd tijd voor me vrij te maken en ben ik denk ik een van de weinige promovendi die bijna iedere week minstens 1 uur overleg heb gehad, en regelmatig zelfs meer, waar naast werk gerelateerde ook persoonlijke zaken besproken konden worden. Ik vind het erg mooi om te zien hoe veel plezier je haalt uit het uitpluizen van zeldzame metabole ziekten (of het Corona virus) en ik hoop dat ik uiteindelijk ook ergens beland waar ik met dezelfde passie mijn werk kan uitvoeren.

Beste Jaap Jan, ook jou ben ik dankbaar voor de afgelopen 3,5 jaar. Zonder twijfel heb ik van jou geleerd 'how to sell your science'. En dat het manuscript altijd nog wel korter kan. Je immer optimistische stemming werkt inspirerend en ik heb met veel plezier toegekeken hoe je een halve beroemdheid bent op de TCT meetings en niet te vergeten je dansmoves! Je vertrek naar NYC was even schakelen, maar ik bewonder je keuze en vind het mooi om te zien hoe je je daar zo thuis voelt. Ook de toevallige connectie met Leontien blijft leuk en ik 
ben jullie beiden dankbaar voor de connectie met Folkert Brijker op Curaçao die me met al zijn enthousiasme overtuigd heeft voor mijn eerste klinische baan naar het Curaçao Medical Center te gaan.

Beste Stefan, ook zonder jou was dit proefschrift er nooit geweest. Althans, niet in zijn huidige vorm en met mijn naam er onder. Jouw vertrouwen en stimulans heeft mij 3,5 jaar geleden na mijn wetenschapsstage doen besluiten te solliciteren voor dit promotietraject, tot ongeloof van heel veel mensen haha. Ondanks dat het proefschrift uiteindelijk slechts een enkel hoofdstuk immunologie bevat, ben je altijd betrokken geweest en ben ik volledig opgenomen in de BoNi groep waar ik veel steun aan heb gehad tijdens het hele traject. Ik vind het knap hoe je altijd het positieve kan blijven zien en hoe je met gemak de meest ingewikkelde immunologische theorieën kunt uitleggen (en kunt blijven herhalen). Naast Stefan, heb ook jij Regina, mij tijdens mijn wetenschapsstage dat laatste zetje in de rug gegeven en vanzelfsprekend ben ik ook jou daar erg dankbaar voor.

Beste Nanda, toen pas later in het traject duidelijk werd dat het proefschrift toch wel meer metabool gericht werd in plaats van immunologisch ben jij, als professor metabole ziekten, gevraagd als mijn promotor op te treden. Ik ben je erg dankbaar dat je die taak op je wilde nemen en ook voor je meer lab-gerichte input die ik zeker goed kon gebruiken. Ik ben tevens toch wel trots dat je me tot een van de meisjes-met-lijstjes hebt bestempeld, aangezien dat namelijk niet persé mijn sterkste eigenschap is ;).

De leden van de beoordelingscommissie, Prof. Dr. N.M. Wulffraat, Prof. Dr. K.P.J. Braun, Prof. Dr. P.M. Hoogerbrugge, Prof. Dr. C.D. van Karnebeek, en Dr. J.J.M. Jans wil ik graag bedanken voor hun flexibiliteit, tijd en bereidheid mijn proefschrift te beoordelen.

Beste Sylvia Toth, misschien had ik wel met u dit dankwoord moeten beginnen. Zonder de schenking van de Sylvia Toth Charity Foundation was dit proefschrift er zeker niet geweest. Ontzettend bedankt dat $\mathrm{u}$ zich inzet voor deze groep patiënten. Ik hoop dat de Foundation nog lang mag blijven bestaan en zich o.a. voor dit soort doelen blijft inzetten!

Alle artsen en assistenten uit het UMCU die hebben meegewerkt aan de papers wil ik bedanken voor hun specifieke input. Michelle van Egmond-Ebbeling en Jens Achterberg dank voor alle hulp bij het begrijpen van het duizelingwekkende aantal afkortingen bij de oogheelkunde en natuurlijk jullie input voor de oogheelkunde paper. Diane Smit, bedankt voor jouw kennis, inzet en pragmatische manier van denken bij het hearing loss stuk, bij uitstek het meest efficiënte stuk dat ik geschreven heb en misschien wel ooit ga schrijven. Alle metabole specialisten, Klaas Koop, Gepke Vissers, en Sabine Fuchs, dank voor alle input die jullie geleverd hebben en voor de supervisie bij de Toth patiënten. Het team van het Sylvia Toth Centrum, Mariëlle, Linda, en Ellen, met veel plezier heb ik altijd de patiënten 
op het STC gezien. Mariëlle, bedankt voor al je inzet om deze dagen zo goed mogelijk te laten verlopen voor de patiënten. Linda en Ellen, helaas hebben we afscheid van jullie moeten nemen maar ik wens jullie het beste op jullie nieuwe plekken! Ook alle andere specialisten betrokken bij het STC, dank voor jullie input, ik heb veel van jullie geleerd! Willemijn en Hanneke, het was fijn om 2 andere metabole PhD'ers te hebben in dezelfde fase van hun traject. Bedankt voor de koffies, lunches en meetings, en de gezelligheid op de congressen/symposia! Ten slotte ook nog het team van de kinderstamceltransplantatie unit, met name Caroline Lindemans en Corinne Gerhardt. Dank voor al jullie hulp en input en uiteraard ook de gezelligheid. Corinne, Salt Lake City was mede dankzij jou een zeer geslaagd eerste congres, dank voor je steun op de eerste rij toen ik met veel te veel zenuwen mijn eerste presentatie moest geven in die veeeel te grote zaal. Caro, ik heb denk ik al mijn transplantatiecongressen met jou mogen bezoeken. Dank voor je gezelligheid, alle info over de mensen die Jaap Jan steeds maar aanspraken en dat ik door jou meerdere keren in de mooie hotels heb mogen verblijven $:$ ! Ik heb ontzettend veel bewondering hoe jij je werk en privé leven weet te combineren.

Uiteraard wil ik ook iedereen van het laboratorium van de Metabole Diagnostiek in het UMCU heel erg bedanken voor jullie hulp en input. In het bijzonder Jaap, dank voor ál je hulp bij de experimenten in het lab. We moesten even wennen aan elkaar maar ik ben je ontzettend dankbaar dat je mijn lab skills hebt getransformeerd van incapabel naar capabel. Een groot deel van de stukken in dit proefschrift waren er zonder jou niet geweest. Heel veel plezier met je pensioen, geniet van al je vrije tijd! Ans en Miriam, jullie natuurlijk ook bedankt voor alle hulp bij de enzymexperimenten, Astrid bedankt voor het beantwoorden van al mijn mails en je hulp bij het uitzoeken van de samples. Ten slotte, Isa en Berthil, jullie ontzettend bedankt voor de hulp bij de GAG metingen en voor de input bij de saliva paper.

Lieve Boni's, Coco, Celina, Jurgen, Rick, Vania, Bas, Shanice, Annelisa, Linde, Maud, Niek, Collin, Esther, Denise, en alle studenten (in het bijzonder Vivian, Doris en Konradin), jullie hebben mijn promotietraject echt een stuk leuker en aangenamer gemaakt. Dank voor alle input en discussies ondanks dat sommigen zich met totaal andere onderzoeksgebieden bezig houden. Annelisa, super bedankt dat je mijn paranimf wilde zijn. Je bent echt een wetenschapper in hart en nieren en het feit dat we je daarom soms belachelijk maken, benadrukt alleen maar meer hoe jaloers we daar eigenlijk op zijn. Ik vind het super mooi om te zien hoe je je ontwikkelt en ben ervan overtuigd dat we je over een $\mathrm{x}$ aantal jaar moeten aanspreken met Professor Cornel! Dank voor al je gezelligheid en lieve woorden. Vania, sometimes you meet someone and you just know you'll be friends! Thanks for all the coffees, alllll the food (I owe you so much), for showing me the best Italian places in Utrecht, and of course the advices concerning Adam, Adam 2.0, and Adam 3.0 (how many more will there 
be?!). I can’t wait to visit your Calabria region where you always speak so fondly of! Coco en Celina, dank voor alle hulp in het lab al tijdens mijn wetenschapsstage en voor de gezelligheid tijdens onze road- en ski trip in Utah, Jurgen voor al je kennis omtrent het wielrennen en je hulp met R (zelfs toen je al uit dienst was), Rick voor je gezelligheid op congressen, Bas voor je input en het sparren over statistiek, Shanice als roomie en mede metabole onderzoeker voor al je input op meerdere vlakken, Linde, Maud, Niek, Collin, Esther, Denise, Doris en Konradin voor alle input tijdens de meetings en iedereen uiteraard voor alle support en gezelligheid! Ook bedank ik graag de sunshine room voor het beschikbaar stellen van jullie koffie en DE stoel wanneer ik even een pauze nodig had. Vivian, jouw hulp bij de review is echt van grote waarde geweest. Ik ben blij te zien dat je masterstage zo goed gaat en wie weet ga je hierna zelf wel door als PhD'er?

Ook mijn kamergenoten Shanice, Kirsten, Arianne, Toine, Peter, Febby, Marjolein, Chilam, Geert, en Alberto wil ik graag bedanken voor de fijne sfeer in onze goed verstopte kamer. Als het nodig was, om successen of frustraties te delen of gewoon zomaar, was er altijd wel iemand in voor een praatje, maar in tijden van stress kon er ook heel hard gewerkt worden. Hopelijk krijgen jullie een goede vervanger op mijn plekje achter de deur. Daarnaast natuurlijk alle andere leden van het LTI-applied. In het bijzonder Sanne, van collega naar fietsmaatje bij CS030 en natuurlijk als roomie op Mallorca. Heel heel erg bedankt voor al je hulp bij de lay-out van dit boekje! Eveline en Julia onwijs bedankt voor al jullie hulp rondom het OLINK project. Eveline, ik ben ervan overtuigd dat als jij er niet was geweest, die samples nog steeds niet waren gemeten haha (als een boer met kiespijn). Verder iedereen bedankt voor alle samenwerkingen, inspirerende projecten, presentaties, feedback, en uitjes, en dat jullie mij als 'zo'n arrogante dokter die geen pipet kan vasthouden' hebben geaccepteerd binnen de groep ;). Hopelijk heb ik bewezen dat dokters ook gewoon gezellige mensen zijn.

Alle leden van de Onderwijs- \& Onderzoeksraad, in het bijzonder Linda, Wynand, Marco, Dorien, Lisanne, Marlinde, Stijn, Pim, Anke, Kristin, Jan en alle anderen bedankt voor de ontzettend leerzame tijd! Het was bijzonder om mee te mogen denken over een aantal uitgebreide complexe beleidsstukken. Hetzelfde geldt voor de MD PhD sensorgroep, onze koffiemeetings waarbij we een aantal belangrijke onderwerpen omtrent promovendi op de juiste tafel hebben weten te leggen hebben me veel voldoening gegeven, dank daarvoor!

Naast alle mensen uit Utrecht wil ik ook graag de Metabollies, Gé-Ann, Laura, Mendy, en Stephanie uit Amsterdam bedanken voor de gezelligheid op de metabole congressen en symposia. Natuurlijk wil ik ook alle deelnemers van het team van de Metabole Specialisten bij de Stofwisseltour bedanken, in het bijzonder Simon, Hanneke, Johanneke, Leo, Henk 
en Josina, Herman, Hans en Yne, George en Mariska, Nanda en Pieter, en Ronald. We hebben met zijn allen heel wat kilometers en uren in het zadel door gebracht. Ik bewonder met hoeveel passie en enthousiasme jullie je inzetten om geld op te halen voor meer onderzoek naar deze vreselijke aandoeningen want dat is hard nodig!

Naast directe collega's zijn er ook een aantal mensen buiten werk die het leven een stuk aangenamer maken. Ook hen wil ik graag in het zonnetje zetten en kunnen dus niet ontbreken in dit dankwoord:

Alle matties van de Epi-master en in het bijzonder Cilie, Tamar, Aernoud, Joline, Rutger, Marieke, Bianca, Katrien, en Dirk-Jan. Ik vond het heerlijk weer even korte termijn resultaten te kunnen behalen tijdens mijn promotietraject en ben jullie dankbaar voor alle borrels, koffies, lunches, diners in de Brink, gesprekken om te sparren over methodologie en niet-epi gerelateerde zaken, en, niet te vergeten, alle hulp bij R!

Natuurlijk ook iedereen die meedeed met Insanity, in het bijzonder Joyce, Inge, Marloes, Marieke, Eef, en de man waar het uiteindelijk allemaal om draait: Shaun T. Van het lege zaaltje in het CalHosp naar de gymzaal van het PMT. Ik ben nog nooit zo fit geweest in mijn leven als nu. Joyce, met name die laatste 2 maanden die hard iedere ochtend om $6.30 \mathrm{u}$ hebben me het ritme en de energie gegeven om de eindstreep te kunnen halen. Ik vind je een geweldig mens en jouw open blik naar alles en iedereen vind ik ontzettend inspirerend. Onwijs bedankt en don't forget: don't throw the towel in the ring, you pick up that towel and use it to wipe of the sweat! Come on y'all, you.just.have.to.dig.deeper! Jump from the bottom! Josh.......

Iedereen van CS030, in het bijzonder Frederieke, Maikel, Jette, Lisanne, Marieke, JanJoost, en Sanne, slecht voor m'n bankrekening, maar goed voor m'n gezondheid, zowel fysiek als mentaal. En tja, wat is nu belangrijker? Bedankt dat jullie me mede hebben laten inzien dat er meer is dan de muren van een ziekenhuis. Jette, bedankt dat je me hebt geleerd te genieten van het opfietsen van een berg. Toch blijf ik erbij dat niets boven een zo snel mogelijke afdaling gaat. Uiteraard ook bedankt Frederieke, Judith en Iris voor onze onovertreffelijke podiumervaring op internationale tv;)!

(Oud) bewoners van de VLSS 1! Appie en Lau, waar zou ik zijn zonder jullie? Van het steeds maar weer openen van de voordeur omdat ik m'n sleutels weer eens vergeten was (en Ap er dus maar een app voor maakte voor op onze telefoons) tot het superviseren terwijl ik de printplaat van m'n wasmachine soldeerde (nogmaals sorry Ap dat ik je per ongeluk elektrocuteerde). Ik vind het jammer dat jullie ons verlaten hebben, maar wens jullie heel veel plezier in jullie nieuwe huis! Gelukkig is de andere helft van de van Westrhenen's er nog: Marleen, ik heb geen moment spijt gehad van het instemmen op de vraag of het oké 
was dat er meerdere mensen uit 1 familie in ons huis woonden. Ik vind je praktische insteek heerlijk en ben mega jaloers op je pannenkoekenplanten die ik zelf niet in leven kan houden! Last but not least, Jochem, knap hoe jij iedereen in het huis hebt weten te verleiden tot het kopen van een eerste dan wel een nieuwe racefiets (inclusief jezelf). Ik heb onwijs veel van je geleerd (en nog steeds!) en bewonder je toewijding en kennis. Ik heb er alle vertrouwen in dat het afronden van je scriptie goed gaat komen en hoop dat je daarna een baan vind waar je zowel uitdaging als plezier uit weet te halen!

Eelco en Michiel, nog net geen jut en jul of buurman en buurman. Ik heb ontzettend genoten van onze koffietjes en ben blij dat ons laatste jaar van Geneeskunde zich heeft voortgezet in het UMCU met als hoogtepunt natuurlijk ons fietstripje naar Brabant en Gelderland. Uiteraard Eelco, haal ik hier nog ééééén keer aan hoe jij gewapend mét laptop de heuvels steeds stiller omhoog ploeterde met al dat extra gewicht, want 'hij ging nog wel wat aan z'n onderzoek doen' (niet dus, want de laptop bleek leeg te zijn). Ook al zie je het zelf misschien niet altijd, ik ben ervan overtuigd dat jij een dijk van een proefschrift gaat uitgeven komend jaar en je mag trots zijn op wat je allemaal hebt bereikt! Ik hoop dat onze vriendschap hier niet ophoud en we de koffies voortzetten voor meetings in de kroeg. Michiel, ik heb onwijs veel respect hoe jij je promotie aanpakt en omgaat met alle uitdagingen. Jouw positieve houding werkt aanstekelijk en ik heb genoten van mijn bezoekje aan het mooie Oxford. Ik ben blij te kunnen zeggen er een goede vriend bij te hebben en hoop dat dat nog lang zo blijft!

Lieve Najaden, jullie natuurlijk bedankt voor álle donderdag-etentjes, activiteiten, borrels, en uiteraard adviezen. Ook al neemt de frequentie als oud-lid wat af, het is nog steeds een feestje om bij jullie aan te haken. Babs en Stees, beste mede oud-bestuursleden ooit, hopelijk blijven we elkaar nog lang zien! Miek, AL, en Lau, ik ben blij dat hier een hechte vriendschap is ontstaan en ben supertrots op de paden die jullie allemaal gekozen hebben. Johanneke, dank dat ik van jou de eerste beginselen van het promoveren heb mogen leren en voor alle koffiemomenten, de adoptie binnen de Frank's Angels groep en uiteraard alle fietsavonturen!

Lieve Sexy Club 7, Iris, Anne, Natanja, Naomi, Sanne, Willy \& Appendici, mijn grote voorbeelden wat betreft huizen kopen, trouwen en baby's! Ik ben trots dat iedereen zo goed op weg is naar het 'zijn van de dokter die je wilt zijn' en ik geniet van onze heerlijke geneeskundepraat gewoon omdat het kan, het liefst samengaand met een glas wijn en een goede kaas / charcuterie plank. Ik bewonder ieder jaar weer de meest geweldige creaties die voorbij komen tijdens het nieuwjaarsdiner en ik ben benieuwd hoe snel de huisjes voor ons jaarlijkse weekend groter moeten gaan worden! 
Lieve Betties, Moos, Buus, Saar, Dien, Liek, Lidie, Jen, Em, Mel, en Merel, mijn Brabantse vriendinnen in hart en nieren. Ieder jaar weer kan ik op jullie rekenen tijdens het mooiste feest van het jaar: CARNAVAL! Ik vind jullie stuk voor stuk prachtige mensen met persoonlijkheden waar je U tegen zegt. Ondanks dat we allemaal compleet verschillend zijn, voelt het altijd vertrouwd als we samen zijn. Onze vakantie op Sicilië voor Jens bruiloft heeft dat maar weer bewezen. Ik ben trots op wat jullie allemaal bereikt hebben en ik hoop dat we elkaar nog vele vele jaren blijven zien en wie weet komen we Mussi dan ook nog steeds tegen.

Bas, hier issie dan eindelijk: de enige echte Bridget Jones' Diary. Hopelijk vind je de omslag mooi genoeg om hem niet met zwarte marker te bekladden. Ik vind het mooi om te zien hoe je je eigen pad bewandelt en je jezelf steeds beter leert kennen samen met Lieke. Ik ben blij dat we elkaar niet uit het oog verloren zijn en mega dankbaar dat je het aandurfde om met 4 meiden op motorvakantie naar de Indiase Himalaya te gaan. Where next?

Lieve Iris, vanaf dag 1 in Utrecht was het dikke mik. Onze tijd als huisgenoten op de Zaagmolenkade heeft dat alleen maar bevestigd. Ik vond het heerlijk om bij jou en Stefan op de bank te ploffen en ben jullie eeuwig dankbaar voor de heerlijke maaltijden en baksels die ik voorgeschoteld heb gekregen. Toch zijn jullie waarschijnlijk blij dat jullie 's nachts geen dronken eigenwijze persoon meer de verkeerde kamer binnen zien komen strompelen en ik dat ik al tijden geen vreemde weddenschappen meer heb afgelegd met Stefan. Ondanks dat onze levens op het moment compleet verschillend zijn, ben ik ontzettend blij dat we zo'n goed contact hebben. Ik vind je een geweldige moeder voor Lize en Hugo, tegelijkertijd ben je een fantastische vriendin met altijd goede raad als ik m'n hart kom luchten en ik hoop dat dat nog heel lang zo zal blijven.

Lieve JanB, mijn psycholoog on speeddial. Onwijs bedankt voor al je steun de afgelopen jaren. Het is inmiddels alweer zo'n 10 jaar geleden dat jij naast Henk en Dia bij ons op de Cornelis Dirkszstraat kwam wonen. Ik ben blij dat we elkaar daarna niet uit het oog verloren, maar juist naar elkaar toe gegroeid zijn. Kan niet anders dat de basis daarvan is gelegd toen jij de wekelijkse GTST updates naar me mailde toen ik op reis was ;). Ik ben ontzettend trots op hoe je de afgelopen jaren in het leven hebt gestaan en ben ontzettend blij om te zien dat je het nu zo fijn hebt met Guido. Ik geniet volop van al onze momenten waarin we ieder detail kunnen overanalyseren maar we daar uiteindelijk ook gewoon om kunnen lachen. Ik hoop dat er nog vele koffies, lunches, etentjes, belletjes, gaypride's, etc. etc. blijven volgen en ik ben je eeeeeeuwig dankbaar voor de TV en het koffiezetapparaat die je kwam brengen tijdens mijn Corona-thuisquarantaine! 
Lieve Mieke, vanaf moment 1 dat ik bij Najade hoorde was ik gefascineerd door de energie die jij hebt. Toen ik me vervolgens bij jou en Annelinne aansloot in Thailand was een hechte vriendschap geboren. Jij de Yin, ik de Yang en vice versa. Wat meestal goed gaat en soms botst (als ik te veel zooi maak of jij naar mijn mening te vroeg over details nadenkt). Jarenlang waren we het dreamteam in de kroeg en op festivals maar ook op reis of tijdens het sporten. Je weet me altijd te stimuleren iets juist wél te doen (a.k.a. wat heb je te verliezen?) en ik ben je dankbaar voor alle herinneringen die we samen hebben gemaakt. Ik ben super trots op hoe je je ontwikkelt hebt tot de persoon die je nu bent. Samen met Coen heb je echt het allermooiste baby'tje Hessel ooit op deze wereld gezet en ik heb nu al zin in alle nieuwe herinneringen die we nog gaan maken (en de fotoboeken die jij daar vervolgens weer van gaat maken hehe ;)).

Lieve Moos, het is niet voor niets dat jij nu als paranimf naast me staat. Al sinds het Norbie ben je overal bij geweest en ik ben ontzettend blij dat ons vertrek naar Utrecht onze vriendschap alleen maar heeft doen groeien. We kunnen als de besten samen lachen, úúúuren situaties en gedrag analyseren en ook als het een keer wat minder gaat, sta je voor me klaar. Bij jou voel ik me altijd gewaardeerd en ben ik goed zoals ik ben. En dat is precies wat ik zo aan je bewonder. Jij ziet altijd het positieve in iedereen en weet dat ook in mensen naar boven te halen. Ik ben super trots op jou als persoon en ik hoop, maar ben er ook van overtuigd, dat je uiteindelijk een plek vindt waar je die kwaliteiten maximaal kan inzetten. Geen idee hoe de verzorgingstehuizen er over 50 jaar uitzien, maar laat ze de tafels maar vast aan de kant zetten, want de polonaise zullen we daar samen lopen! Dankjewel dat je altijd mijn grootste fan bent, ik zou oprecht niet weten wat ik zonder je moest $\odot$.

Vrienden kies je zelf, je familie moet je het mee doen. Frenk en Angeline officieel gezien eigenlijk geen familie, maar ik had me geen betere peetoom en peettante kunnen wensen. Als kind verwend met cadeau's en uitstapjes, tegenwoordig met oude verhalen en foto's over/van onspa. Alhoewel soms confronterend, ik geniet volop van de motortochtjes met Frenk op de trouwe Yamaha van Angeline. Binnenkort ga ik echt zelf die motor aanschaffen en volgen er hopelijk nog velen tochten door binnen- en buitenland! Dank dat jullie er altijd voor me zijn.

Anita, als er iemand een voorbeeld voor me is geweest dan ben jij het wel. Van de reislust, tot het ziekenhuis, ik vind het allemaal bij je terug. Dankjewel voor álles wat je voor me gedaan hebt! En hoewel dat kasteel weinig prioriteit meer heeft, ben je zeker nog altijd welkom in een kamertje als bejaarde. Mocht je tegen die tijd meer ruimte nodig hebben, moet je denk ik Y lief aan gaan kijken ;).

$\mathbf{Y}$, de grote zus die altijd over me waakt of ik nu wil of niet. Hoewel we vroeger nog geen 20 minuten in dezelfde ruimte konden zijn, hebben we dat later meer dan goed gemaakt. Onze reizen door India en Zuid-Amerika zijn daar goede voorbeelden van. Ik ben je ontzettend 
dankbaar dat je er altijd voor me bent, me helpt mezelf beter te begrijpen en om te gaan met moeilijke situaties. Ik bewonder je om je enorme EQ en hoe je altijd overal kansen ziet en die ook volledig benut. Ik hoop dat je ontzettend gelukkig gaat zijn in je prachtige nieuwe huis en in je leven samen met Ufuk.

Nicolleke, de vrolijkste zus van ons drieën! Ik vind het geweldig om te zien hoe jij je leven samen met Erik zo goed vormgeeft. Het was voor jou niet altijd makkelijk in de fam en je hebt heel veel teleurstellingen moeten verwerken. Het feit dat je desondanks de vrolijkste bent zegt dan ook heel veel over jou als persoon, iets waar ook ik veel van kan leren. Ik ben ontzettend trots op hoe je het doet en op wie je bent en ik hoop heel erg dat jullie grote wens uit mag komen.

Mam, we hebben zo onze goede en minder goede tijden gehad. Ik ben blij dat we tegenwoordig op goede voet met elkaar staan en er ruimte is om elkaar te begrijpen. Mario is hier denk ik zeker een belangrijke factor in geweest en die ben ik daar ook meer dan dankbaar voor. Ik begin steeds meer in te zien hoe veel ik op je lijk. Wie had gedacht dat ik dat ooit zou zeggen $\odot$. Je hebt een hoop voor je kiezen gekregen, maar sterk als je bent, heb je je leven weder opgebouwd en zie ook ik nu de leuke, gezellige, lieve, zorgzame, maar zeker ook ondernemende vrouw in je. Bedankt voor alle ruimte die je me gegeven hebt die ik nodig had om dat in te zien. Ik bewonder je hoe je je nu bekommert om al die oudjes en wie weet lopen we echt nog een keer die Vierdaagse samen.

Ten slotte, lieve $\mathbf{P a}$, het is ongelooflijk dat het al 15 jaar geleden is dat $\mathrm{Y}$ en ik daar in de serre jouw handdruk voelden verslappen en onsma, Y, Nicolleke en ik nog maar met 4 over waren. Veel te vroeg uiteraard, maar gelukkig hadden we toen je vastberadenheid al overgenomen en had je ons al geleerd om altijd 3 stappen vooruit te denken. Om die Engelse tang al klaar te hebben liggen als jij nog met de kruiskop schroevendraaier bezig was. Een geschenk en vloek tegelijkertijd weet ik nu, aangezien niet iedereen altijd op die manier met me mee denkt. De afgelopen 15 jaar zijn zeker niet altijd makkelijk geweest. Maar zoals je ziet, je 4 meiden doen het goed. Meer dan goed. Je kan trots op ons zijn. Ik ben trots. Op ons. En op jou $<3$. 
\begin{tabular}{l|l} 
Dankwoord & 225
\end{tabular} 



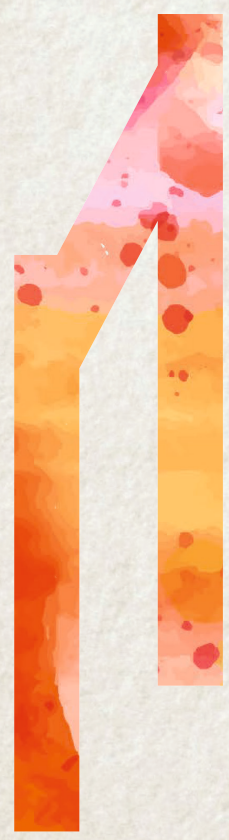

$\operatorname{los}_{\rightarrow 02}$

an+ $\times 2$

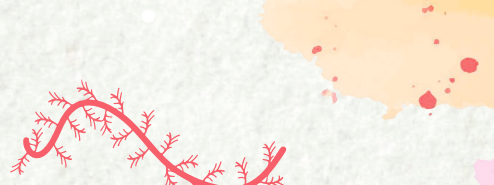

तो $x$

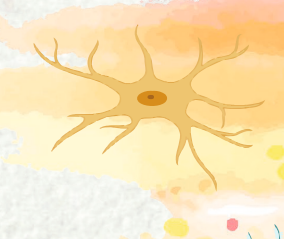

समिए

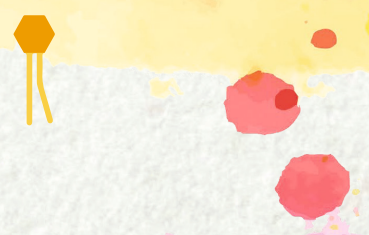

त) $x$ 